3D ANALYSIS OF HIP JOINT MOBILITY AND THE EVOLUTION OF LOCOMOTOR ABILITIES IN MIOCENE HOMINOIDS

\author{
A Dissertation \\ Presented to
}

The Faculty of the Graduate School

at the University of Missouri

In Partial Fulfillment

Of the Requirements for the Degree

Doctor of Philosophy

\author{
By \\ ASHLEY S. HAMMOND \\ Dr. Carol V. Ward, Dissertation Supervisor
}

December 2013 
(C) Copyright by Ashley S. Hammond 2013

All Rights Reserved 
The undersigned, appointed by the dean of the Graduate School, have examined the Dissertation entitled

3D ANALYSIS OF HIP JOINT MOBILITY AND THE EVOLUTION OF LOCOMOTOR ABILITIES IN MIOCENE HOMINOIDS

Presented by Ashley S. Hammond

A candidate for the degree of

Doctor of Philosophy

And hereby certify that, in their opinion, it is worthy of acceptance.

Carol V. Ward

J. Michael Plavcan

Gregory Blomquist

Scott Maddux

Casey Holliday 
This work is dedicated to Kristina Aldridge and Cheryl Hill, for their support. 


\section{ACKNOWLEDGMENTS}

I have had the great fortune to work with amazing people these last few years. First and foremost, Carol Ward, who has been a loyal and enthusiastic advisor. Carol's research has guided my own work and I thank her for helping me achieve virtually every goal I had upon entering graduate school. My committee-- Mike Plavcan, Greg Blomquist, Scott Maddux, and Casey Holliday-- deserve special recognition for helping me develop my ideas and articulate them clearly. My committee has been a great strength to me and I can't thank them enough for their support, encouragement, and difficult questions.

All faculty in the Integrative Anatomy group have been instrumental to my success, including Kris Aldridge, Cheryl Hill, Matt Ravosa, Rachel Dunn, and Kevin Middleton. In particular, I am deeply appreciative of Kevin's extreme patience for my never-ending questions related to $\mathrm{R}$ and phylogenetic quantitative methods.

I have countless insightful and generous colleagues which have made this experience more rewarding, and here I name just a few. I consider my field work with Meave Leakey to be one of the most memorable and rewarding times of my life and I cannot fully express how grateful I am for the experience. David Begun and Laszlo Kordos have shown extreme generosity in sharing the Rudapithecus fossils, and I am fortunate to have been given access and permission to study the fossils from Rudabánya. Laura MacLatchy's work has been inspirational and the foundation for much of my own work, and I have greatly valued Laura's advice and feedback at multiple stages during my dissertation. I must thank everyone at the Institut Català de Paleontologia for allowing me to join their field crew for a season and study the original 
fossils under their care. In particular, I thank Salvador Moyà-Solà, David Alba, Isaac Casanovas-Vilar, and Sergio Almécija. I will not soon forget the late nights in Barcelona philosophizing about Miocene ape evolution over beers with David and Sergio.

In addition, all graduate and undergraduate students in the University of Missouri Integrative Anatomy program have enriched my life and my work! I cannot thank our undergraduate research assistants enough for processing what must have seemed like endless amounts of scan data. I sincerely hope that Sarah Swartz, Zach Winkler, and Nik Koscielniak did not find themselves in need of glasses and with chronic ulnar nerve pain from too many hours using PolyWorks. Rachel Menegaz, Kim Congdon, Ian George, Henry Tsai and Elizabeth Moffett have made outstanding grad student colleagues and I look forward to being colleagues with them for years to come. Henry, I thank you for the frequent light-hearted banter and your love of mammals.

In the last year, I have found a place in Anatomical Sciences at Stony Brook University. Even as a graduate student, I have been welcomed as a colleague into one of the most successful, evolutionary-focused anatomy departments in the country. I have valued my interactions with each of the faculty and postdocs, and must particularly thank Bill Jungers, Susan Larson, Brigitte Demes, and John Fleagle for discussions relevant to my dissertation.

I am indebted to the museum curators and collection managers who allowed me to study the collections under their care. I thank Eileen Westwig, Judy Chupasko, Lyman Jellema, Loic Costeur, Lorenzo Rook, Darrin Lunde, Bill Stanley, Dave Hunt, Michael Hiermeier, Steven van der Mije, Emma Mbua, and Kyalo Manthi for facilitating my collection visits. I am also greatly appreciative for the advice and PolyWorks software support offered by Angelia Payne, Jack Cothren, and the Center for Advanced Spatial Technologies (CAST) at the University of Arkansas. 
I have been very fortunate to work with many wonderful veterinarians. Doug Armstrong and Julie Napier allowed me collect pilot data at the Omaha Henry Doorly Zoo. I attribute this early data collection on hominoids as being instrumental to receiving grants later. Everyone at the Omaha Zoo, including Doug and Julie, were incredibly gracious and facilitated my work in every way possible. I must also thank Kathy Brasky, Robert Baker, Chris Hanley, Beth Hammond, Nancy Schultz-Darken, Fawn Stroud, Elizabeth Strobert, and all veterinary staff at the zoos and National Primate Research Centers I have worked with. All of you exposed me to an entirely different perspective than I would ordinarily encounter in my research.

Debbie Allen and Mark Hannink in the MU Life Sciences Fellowship program always expressed enthusiasm for my research and made annual reporting a genuinely fun experience. I thank the administrators and staff at my granting agencies, including Leslie Aiello (Wenner-Gren Foundation), Paddy Moore (LSB Leakey Foundation), and Carolyn Ehardt (National Science Foundation, Award BCS 1232393).

To my friends and family—I couldn't have done it without you and apologize for not being available often these last few years. I have missed birthdays, weddings, holidays, and plenty of good times to work on my dissertation, and I thank you for being so understanding. My parents and my sister Aimee have been incredible parents to Beast (an extremely challenging dog!) while I collected data and, I'm ashamed to admit, for quite some time after data collection was finished. Sergio—-thank you for understanding that dissertations are not supposed to be fun and trying your best to make me have fun anyways. A number of friends hosted me while collecting data, and I must thank Sam Sargeant, Darice Westphal, Erin Dougherty, Susan Dougherty, Dave Leslie, and Alex Townsend in particular for their generosity. Darice Westphal offered me more emotional support than I am probably capable of giving anyone myself, and for that I 
cannot thank her enough. I hope I can repay all of you for your deeds these last few years. 


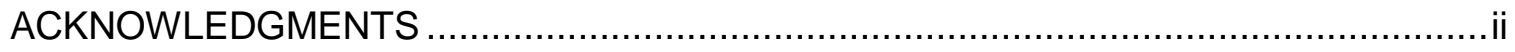

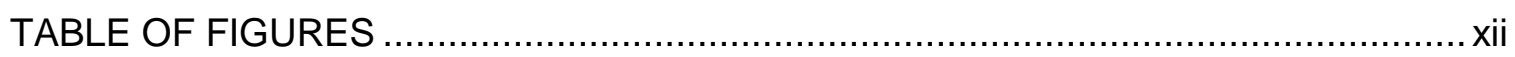

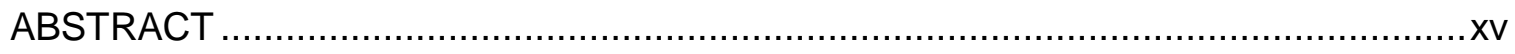

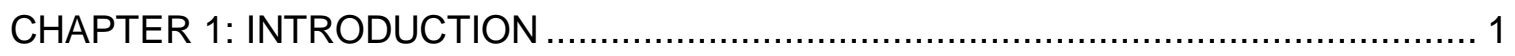

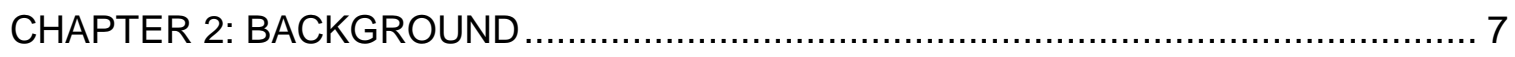

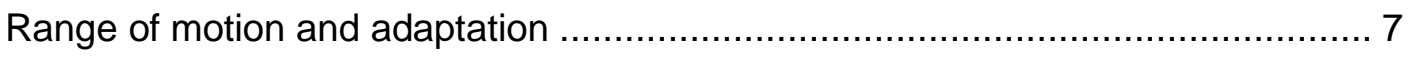

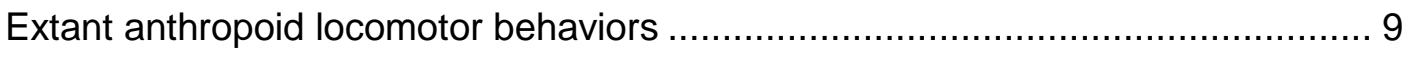

Gibbons (Hylobates) and siamangs (Symphalangus) ................................... 10

Orangutans (Pongo pygmaeus, Pongo abelii) ............................................. 10

Western lowland gorillas (Gorilla gorilla gorilla) and mountain gorillas (G.

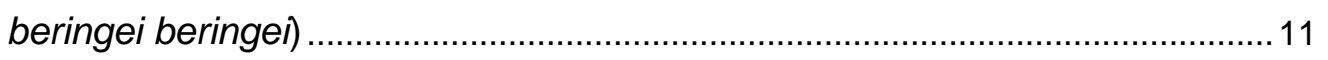

Chimpanzees (Pan troglodytes) and bonobos (Pan paniscus) ......................... 12

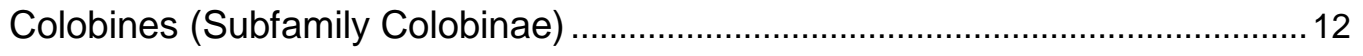

Cercopithecines (Subfamily Cercopithecinae) ............................................ 13

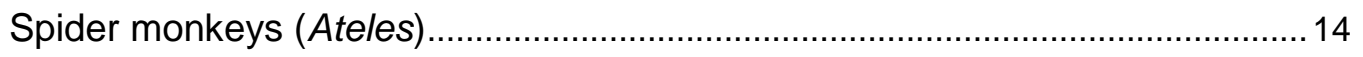

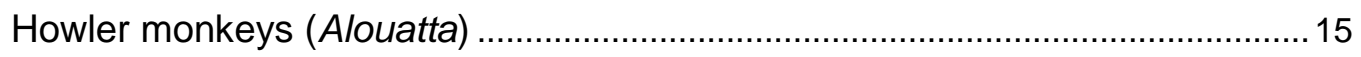

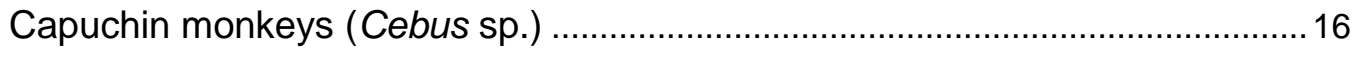

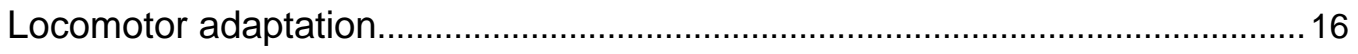

Extant anthropoid hip joint range of motion ...........................................

Influence of soft tissue morphology on hip mobility ....................................20

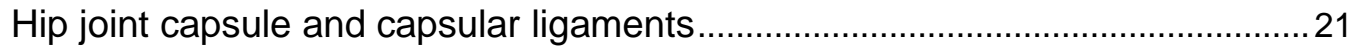

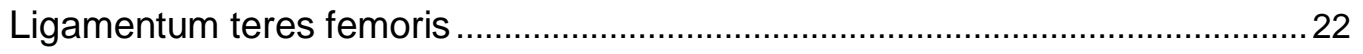

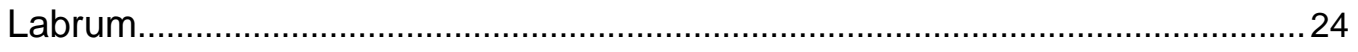


Muscles .

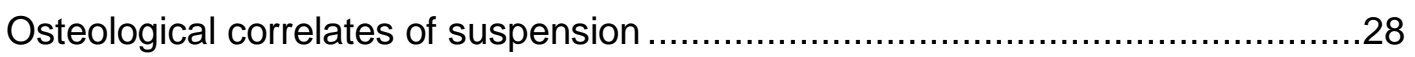

Features of the femur and pelvis attributed to high hip mobility .......................28

Features of the femur and pelvis that increase span at the foot .........................33

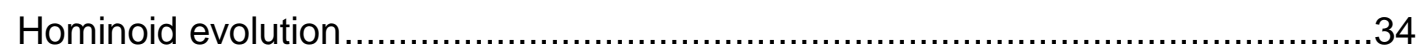

Fossil evidence of locomotor evolution in Miocene apes ...................................... 34

Problems in understanding evolution of fossil apes ........................................ 49

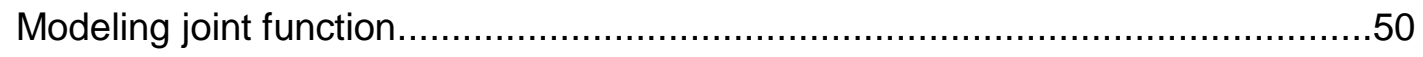

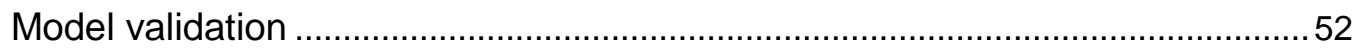

Table 2.1 Comparison of percentages of adult locomotion in extant anthropoids

Table 2.2 Broad locomotor categorization of extant anthropoids considered in

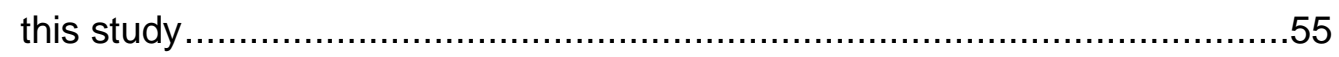

Table 2.3 Comparison of published values for anthropoid hip joint mobility....56

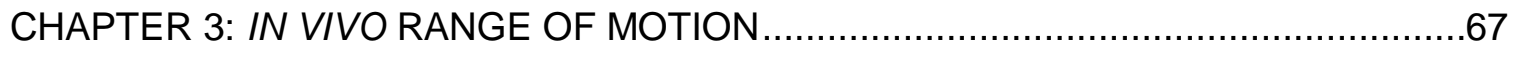

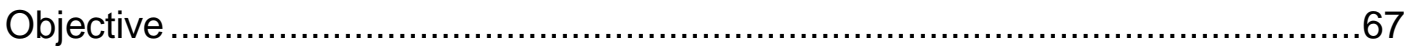

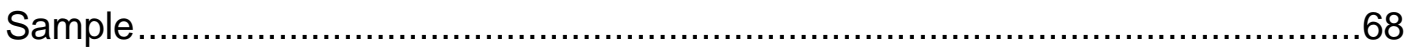

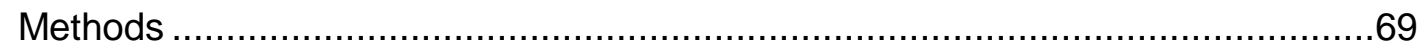

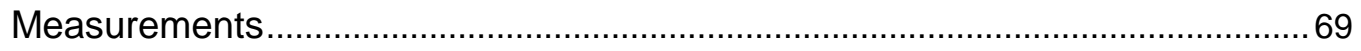

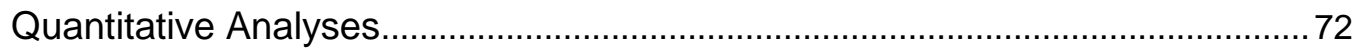

Phylogenetic regression and ordinary least squares regression....................... 73

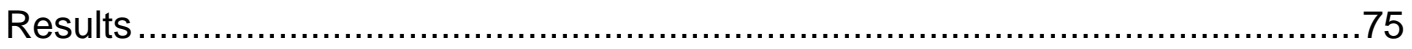

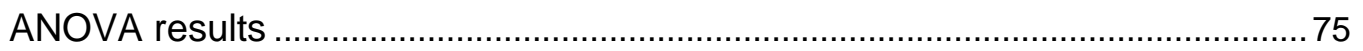

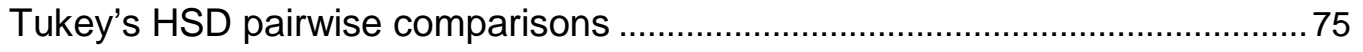

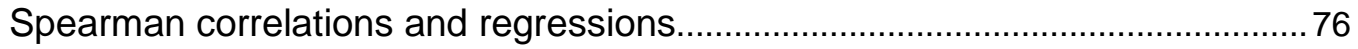


Discussion.

Conclusions

Table 3.1 In vivo sample .88

Table 3.2 Sex-specific descriptive statistics for age and body size..... .89

Table 3.3 Sex-specific descriptive statistics for flexion and extension .90

Table 3.4 Sex-specific descriptive statistics for adduction and abduction ........91

Table 3.5 Sex-specific descriptive statistics for internal and external rotation...

Table 3.6 Sex-specific descriptive statistics for linear dimensions .93

Table 3.7 Species-specific pairwise comparisons for flexion-extension .94

Table 3.8 Species-specific pairwise comparisons for adduction-abduction......95

Table 3.9 Species-specific pairwise comparisons for rotation........................96

Table 3.10 Additional measures of abduction ......................................97

Table 3.11 Species-specific pairwise comparisons for additional measures....98

Table 3.12 Species-specific Spearman correlations and OLS regressions......99

Table 3.13 Phylogenetic regressions (female-specific data only) .................100

CHAPTER 4: IN SILICO MODELS OF HIP JOINT ABDUCTION ABILITY .................107

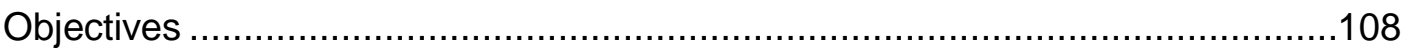

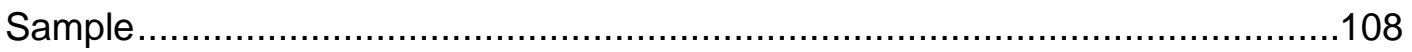

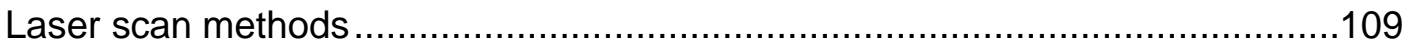

Scanning and polygonal model rendering................................................... 109

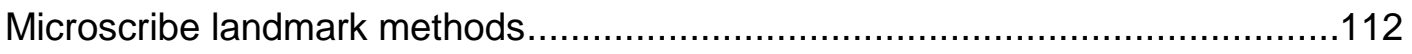

Pelvis articulation methods and landmarks ................................................. 112

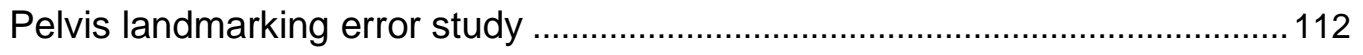

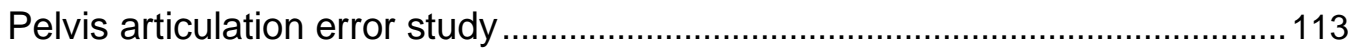




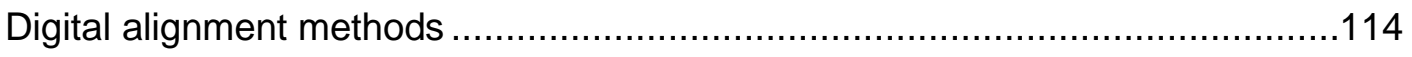

Pelvis alignment to landmark coordinate data............................................... 114

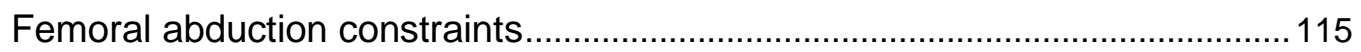

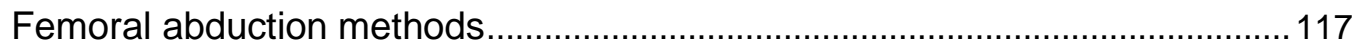

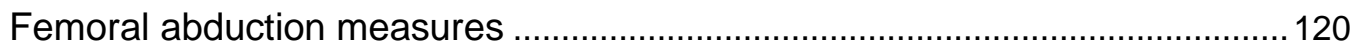

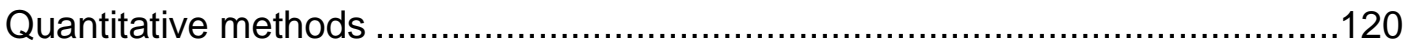

Differences between suspensory and non-suspensory primates.....................120

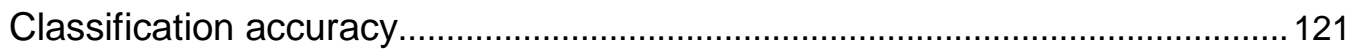

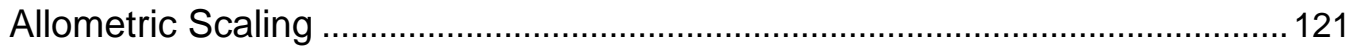

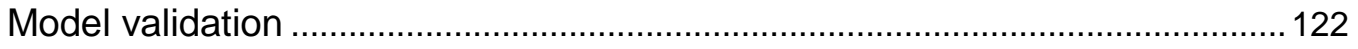

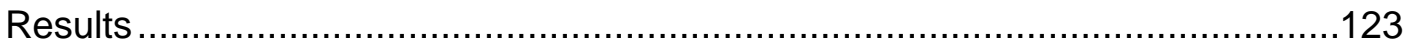

Differences between suspensory and non-suspensory groups in full extant

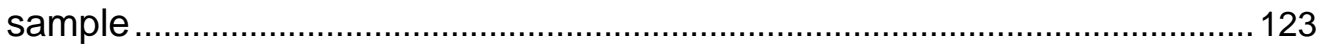

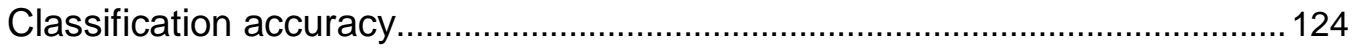

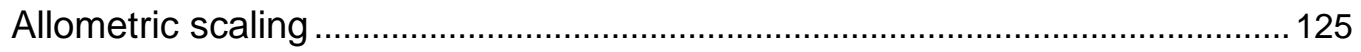

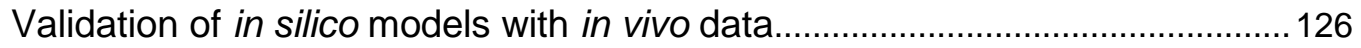

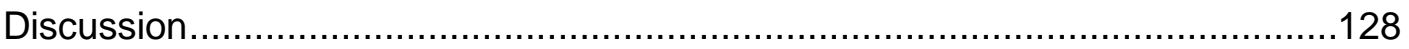

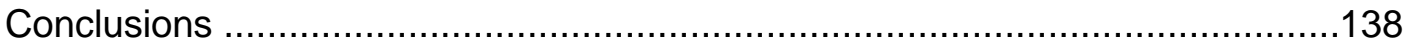

Table 4.1 Extant laser scan and microscribe landmark sample $(n=252) \ldots \ldots . .140$

Table 4.2 Microscribe landmark coordinates ..........................................141

Table 4.3 Intraobserver mean deviation ( $\mathrm{mm}$ ) and error (RMSE) across

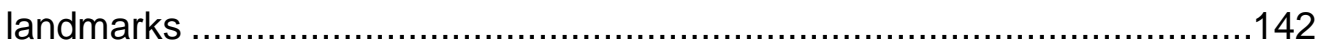

Table 4.4 Interlandmark distances used in pelvis articulation error study ......143

Table 4.5 Interlandmark distances $(\mathrm{mm})$ and mean deviations $(\mathrm{mm})$...........144 
Table 4.6 Differences in 5 repeated trials of best-fit diaphyseal vectors for

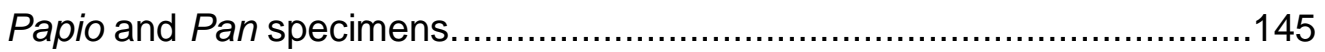

Table 4.7 Sex-specific descriptive statistics for abduction (in silico) .............146 Table 4.8 Percent correct classification in in silico models using discriminant function analysis.

Table 4.9 Sex-specific descriptive statistics for abducted knee position (in silico) 148

Table 4.10 ANOVAs for angular abduction. 149

Table 4.11 Means by locomotor group and treatment type..... 150

Table 4.12 ANOVAs for knee position. 151

Table 4.13 Percent difference between sex-specific means by data type.....152 Table 4.14 Tukey's HSD comparisons between data types (in vivo vs. in silico) for angle of abduction. .153

Table 4.15 Tukey's HSD comparisons between data types (in vivo vs. in silico) for abducted knee position .154

\section{CHAPTER 5: RECONSTRUCTING HIP JOINT FUNCTION IN FOSSIL CATARRHINES}

Fossil taxa and hypothesized locomotor reconstructions 171

Objectives .175

Sample .176

Methods .178

Fossils preserving midline morphology (Proconsul) 179

Fossils without midline morphology (Rudapithecus, Theropithecus, Paracolobus) 180

Quantitative Analyses. 184 
Results .184

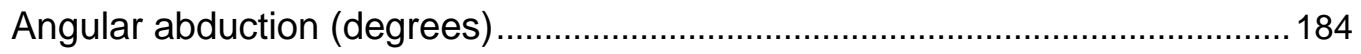

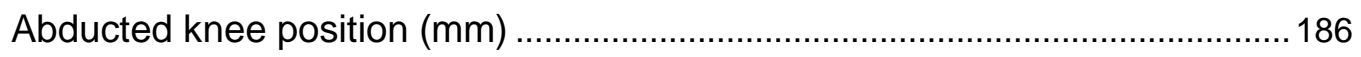

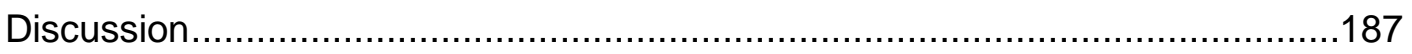

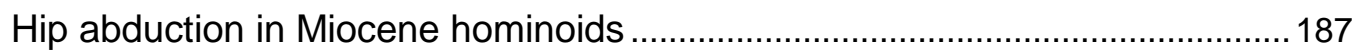

Hip abduction in Plio-Pleistocene fossil cercopithecoids.................................. 190

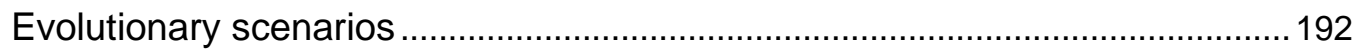

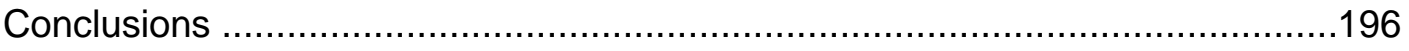

Table 5.1. Fossil sample used in simulations of abduction ........................198

Table 5.2. Procrustes size-removed coordinates from 5 landmarking trials ..199

Table 5.3. Fossil Centroid1 and Centroid2 values from 5 landmarking trials 200

Table 5.4. Extant centroid sizes and specimens used to calculate centroids 201

Table 5.5. Hip function in fossils......................................................202

Table 5.6. Discriminant function percent classification of fossil means in extant suspensory and non-suspensory locomotor groups .........................203

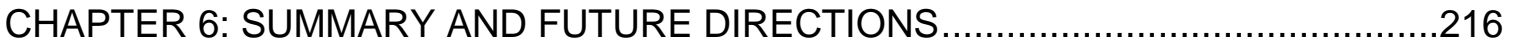

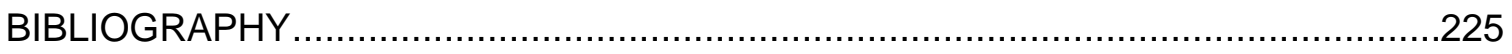

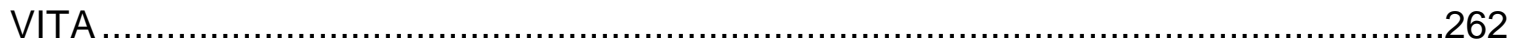

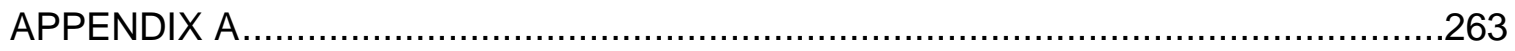

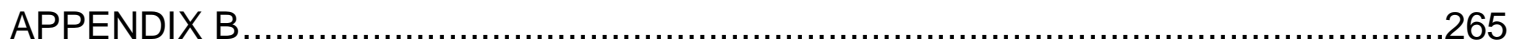

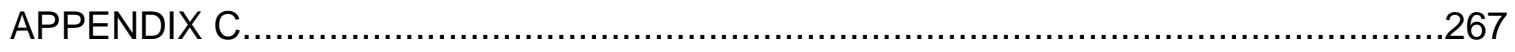




\section{TABLE OF FIGURES}

Figure 1.1. Superfamily Hominoidea with hypothesized positions of fossil apes .......... 6

Figure 2.1 Large hindlimb spatial envelope increases the range of foot positions .........57

Figure 2.2 Hindlimb postures used by anthropoids during different locomotor behaviors

Figure 2.3 Examples of arboreal behaviors used by orangutans .59

Figure 2.4 Phylogenetic relationships between extant taxa with locomotor classification

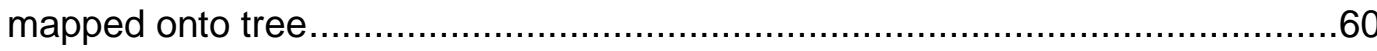

Figure 2.5 Posterior and anterior views of anthropoid femora ...............................61

Figure 2.6 Medial views of anthropoid femora, with an emphasis on femoral head

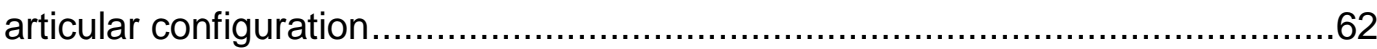

Figure 2.7 An early Miocene hominoid, Proconsul heseloni (KNM-RU 7290 skull and

KNM-RU 2036 skeleton), from Rusinga Island, Kenya

Figure 2.8 A middle Miocene hominoid, Nacholapithecus kerioi (KNM-BG 35250), from

Nachola, Kenya .64

Figure 2.9 Middle to late Miocene hominoids, Pierolapithecus catalaunicus (IPS-21350) and Hispanopithecus laietanus (IPS-18800), from the Vallès-Penedès basin in Spain

Figure 2.10 Late Miocene hominoid, Rudapithecus hungaricus, from Rudabánya,

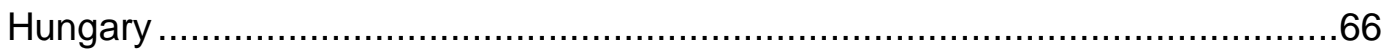

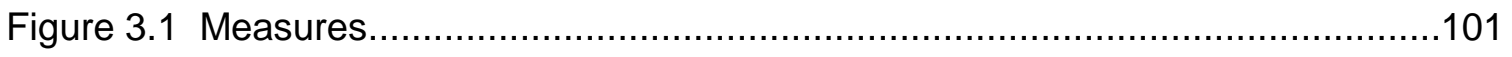

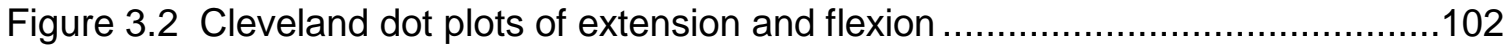

Figure 3.3 Cleveland dot plots for abduction-adduction .................................... 
Figure 3.4 Cleveland dot plots for internal-external rotation.

Figure 3.5 Cleveland dot plots for ranges of flexion-extension, adduction-abduction, and internal-external rotation 105

Figure 3.6 Abduction ability tied to lateral rotation .........................................106

Figure 4.1 Microscribe landmarks are superimposed on a pelvis ..........................155

Figure 4.2 Pelvis polygonal model alignment to landmark data is shown for Papio cynocephalus (OM7276) in the IMInspect module in PolyWorks .....................156

Figure 4.3 The fovea capitis is confined to the boundaries of the acetabular fossa.....157

Figure 4.4 The femoral diaphysis is oriented parallel to an orthogonal plane to the long axis of the ischium 158

Figure 4.5 Femoral head least squares sphere-fitting and centroid creation 159

Figure 4.6 Femoral diaphysis least squares vector-fitting 160

Figure 4.7 Measures of abduction (degrees) and abducted knee position ( $\mathrm{mm}$ ) relative to the midline .161

Figure 4.8 In silico measures of abduction (degrees) ........................................162

Figure 4.9 In silico measures of abducted knee position $(\mathrm{mm})$...............................163

Figure 4.10 Visual comparison of in silico measures of abduction (degrees) and abducted knee position $(\mathrm{mm})$ in a subset of female anthropoids. .164

Figure 4.11 Visual comparison of in silico measures of abduction (degrees) and abducted knee position ( $\mathrm{mm}$ ) in female Gorilla gorilla and Gorilla beringei ......165

Figure 4.12 In silico measures of abduction (degrees) and abducted knee position (mm) on the femoral head sphere diameter using standard major axis regression ....166

Figure 4.13 Comparison of in vivo and in silico data points for angular abduction by locomotor group. .167 
Figure 4.14 Comparison of in vivo and in silico data points for abducted knee position by

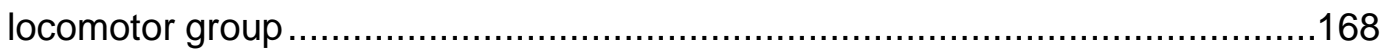

Figure 4.15 Hypothetical examples of the hindlimb spatial envelope .......................169

Figure 5.1. Polygonal models of fossil hominoid and cercopithecoid pelves used in simulations .204

Figure 5.2. Polygonal model of the femur of Proconsul nyanzae (KNM-MW 12142) ...205

Figure 5.3. Polygonal model of the femur of Rudapithecus hungaricus (RUD 184) „...206

Figure 5.4. Polygonal models of the femur of Theropithecus oswaldi (KNM-ER 866,

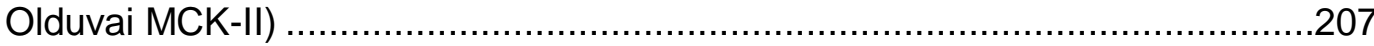

Figure 5.5. Polygonal models of the femur of Paracolobus chemeroni (KNM-BC 3) ...208

Figure 5.6. Polygonal models of the femur of Paracolobus mutiwa (KNM-WT 16827) 209

Figure 5.7. Centroid 2 (using 14 landmarks) as a linear function of Centroid 1 (using 4 landmarks) .210

Figure 5.8. The region of the Rudapithecus pelvis used for acetabular sphere-fitting .211 Figure 5.9. Mean abduction in fossil specimens plotted alongside boxplots of extant taxa

Figure 5.10. Abducted knee position of fossil specimens plotted alongside boxplots of extant taxa

Figure 5.11 Hypothesized sequence of evolution of suspensory behaviors in Miocene hominoids .214

Figure 5.12 Functional abilities within an environmental context. .215 


\title{
3D ANALYSIS OF HIP JOINT MOBILITY AND THE EVOLUTION OF LOCOMOTOR ABILITIES IN MIOCENE HOMINOIDS
}

\author{
Ashley Hammond \\ Dr. Carol V. Ward, Dissertation Supervisor
}

\begin{abstract}
The emergence of extant ape-like locomotor behaviors has become a defining issue in reconstructing ape evolution. Suspensory positional behaviors, such as antipronograde bridging, climbing, clambering and transfer, distinguish extant hominoids from Old World monkeys and most New World monkeys. It has been widely theorized that suspensory behaviors involve highly abducted hip joint postures, potentially permitting suspensory behaviors to be inferred from joint function rather than relying on isolated morphologies. This thesis tests whether adaptations for suspensory behaviors can be inferred in fossil nonhuman hominoids from the hip joint.

The first study tests the association between suspensory behaviors and hip mobility in anesthetized living anthropoids $(n=104)$. Suspensory taxa were found to have significantly higher passive ranges of abduction and external rotation compared to non-suspensory taxa.

The second study developed a digital modeling technique to estimate range of hip abduction and then tested the accuracy of the modeling approach against the live animal data. Hip joint abduction and the abducted knee position were reconstructed in a large sample of extant anthropoids $(n=252)$ and then quantitatively compared these simulations to the in vivo data for passive range of abduction. Suspensory taxa were significantly larger in both simulated abduction (degrees) and abducted knee position
\end{abstract}


(mm), although there was overlap between locomotor groups. The results provided a hypothetical framework for how to interpret abduction modeled in fossil taxa.

The final study modeled hip abduction in early Miocene hominoid Proconsul nyanzae, late Miocene crown hominoid Rudapithecus hungaricus, and several largebodied Plio-Pleistocene fossil cercopithecoids (Paracolobus mutiwa, Paracolobus chemeroni, Theropithecus oswaldi) using the validated modeling approach from the second study. Abduction simulations in Proconsul nyanzae and fossil cercopithecoids yielded abduction consistent with a non-suspensory locomotor reconstruction. Abduction in Rudapithecus hungaricus was exclusively in the range of extant suspensory anthropoids and was most similar to the values observed in spider monkeys and hylobatids. This study provides the first evidence for suspensory behavior in a Miocene ape based on joint function. 


\section{CHAPTER 1: INTRODUCTION}

This thesis addresses whether adaptations for suspensory behaviors can be inferred in fossil nonhuman hominoids (ape taxonomy in Figure 1.1) from the hip joint based on range of abduction. Suspensory positional behaviors are forelimb-dominated behaviors, and include brachiation, antipronograde bridging, vertical climbing, and orthograde clambering and transfer. These behaviors distinguish extant apes from Old World monkeys and most New World monkeys, and presumably primitive early hominoids. The emergence of these extant ape-like locomotor behaviors has become a defining issue in reconstructing ape evolution (recent review in Crompton et al. 2008).

However, we have a poor understanding of how and when the evolution of belowbranch locomotor behaviors occurred. Fossil stem hominid Pierolapithecus (or, hominine per Begun and Ward 2005) seems to lack suspensory adaptations in the hands (Moyà-Solà et al. 2004; Almécija et al. 2009; Alba et al. 2010a), suggesting that suspension evolved independently in hylobatids and great apes. The fossil ape Sivapithecus is probably closely related to the orangutan based on craniofacial similarities yet retains a primitive hominoid body plan, suggesting that great ape-like locomotor behaviors evolved at least twice within great apes (Pilbeam et al. 1990; Ward 1997; Larson 1998; Pilbeam and Young 2001). More recently, it has been argued that the lack of derived features in Ardipithecus ramidus requires that great ape-like locomotor behaviors and morphologies evolved in African apes after the Pan-human split (Lovejoy 2009; Lovejoy et al. 2009a; Lovejoy et al. 2009b). If so, human ancestors 
never passed through an extant great ape-like locomotor stage and somewhat more generalized hominoids may, in fact, be a better model of the more immediate ancestral condition. All of our scenarios about how hominoids evolved depend on an accurate reconstruction of locomotor behavior in apes that lived during the Miocene epoch ( $23-5$ Ma).

The hip joint is hypothesized to be a key site of postural and morphological change during the evolution of hominoid and hominin locomotion. Specifically, below branch behaviors are thought to require a mobile hip joint with a higher range of abduction compared to quadrupedal locomotion, due to the need to reach distantly-spaced discontinuous arboreal supports with the knee and ultimately the foot. This is particularly important for larger-bodied animals using bridging and other suspensory behaviors, rather than leaping behaviors, because large-bodied animals must more cautiously transfer weight from one support to the next (Cartmill 1985). Because these supports are often erratically positioned and oriented, lateral hip abduction abilities are emphasized over the more adducted parasagittal limb movements used when walking on the top of tree branches (Grand 1968; Rodman 1979). In theory, mobility of the hip joint should be the most important hindlimb joint for determining foot position because all movements at the hip joint direct the distal limb segments. Although limb abduction is a frequently cited behavioral component of forelimb-dominated suspensory behaviors (Grand 1972; 1984; Cartmill 1985; Isler 2005; Crompton et al. 2008), it has not been demonstrated that hip abduction abilities differ between suspensory and non-suspensory primates.

Miocene apes have a surprising mix of morphological features of the femur and the pelvis, especially when considered alongside other regions of the postcranium. The 
proximal femur is one of the most frequently preserved hominoid postcranial elements, known from Proconsul (Kenya, 17-20 Ma), Morotopithecus (Uganda, 20.6 Ma), Afropithecus (Kenya, 17 Ma), Equatorius-Kenyapithecus (Kenya, $15 \mathrm{Ma}$ ), Nacholapithecus (Kenya, 15 Ma), Sivapithecus (Pakistan, 9-12 Ma), Hispanopithecus (Spain, 9.5 Ma), c.f. Dryopithecus (Spain, 11.8 Ma), Rudapithecus (Hungary, $10 \mathrm{Ma}$ ), and Oreopithecus (Italy, 7-9 Ma). Despite the high number of Miocene ape femora preserved, we still do not know how to interpret the mosaic morphology in the hip region. For instance, Hispanopithecus has limb proportions and forelimb morphologies that suggest suspensory adaptations (Moyà-Solà and Köhler 1993; 1996; Almécija et al. 2007; Alba et al. 2012; Pina et al. 2012) and yet has a femur that is more similar in shape to other Miocene apes than extant great apes (Moyà-Solà and Köhler 1993; Pina et al. 2012; Almécija et al. 2013). This suggests that suspensory adaptations in largebodied crown hominoids do not always look exactly like the great apes, making locomotor reconstructions based on comparisons to extant bony morphology extremely challenging.

If joint mobility can be reconstructed in fossil hominoids, this would allow us to side-step the issue of how to reconstruct locomotor behavior in fossils with no extant morphological analog. As the articular morphology of the opposing pelvic and femoral joint surfaces should reflect normal limits of motion imposed by soft tissues, it should be possible to reconstruct hip abduction in fossils using just the bones (Jenkins and Camazine 1977; MacLatchy 1996; MacLatchy and Bossert 1996), providing a method for evaluating locomotor abilities in fossil hominoids.

In order to assess whether adaptations for suspensory behaviors can be inferred in fossil nonhuman hominoids, I first test whether suspensory and non-suspensory 
anthropoids actually differ in hip mobility. Chapter 3 presents baseline in vivo measures of hip joint mobility in a large sample of suspensory and non-suspensory nonhuman anthropoids and provides statistical comparisons. Since past workers have emphasized the role of abduction in suspensory behaviors, attention is given to range of abduction and the functional outcome of abduction - the position of the knee relative to the midline. The results presented in Chapter 3 provide compelling evidence that abduction is significantly greater in suspensory anthropoids.

Next, I develop a digital (in silico) model for estimating hip joint abduction using the bony morphology of the pelvis and femur. Hip abduction was reconstructed in Chapter 4 using surface scans of the pelvis aligned to the midline plane and articulating the femur in maximal hip abduction, building on digital modeling parameters outlined by MacLatchy (1995, 1996, 1998) and MacLatchy and Bossert (1996). As in the live animals reported in Chapter 3, abduction ability is significantly increased in suspensory anthropoids when modeled digitally. The in silico models are then validated (following definition from Hutchinson 2011) against live animal measures to provide an estimation of how the models deviate from empirical measures of abduction. This approach provides a model of hip abduction that reliably classifies locomotor group in extant taxa and has a known predictable relationship with empirical measures of joint mobility.

Abduction was then estimated for early Miocene hominoid Proconsul nyanzae (KNM-MW13142) from Kenya and late Miocene Rudapithecus hungaricus (unpublished) from Hungary. These are the only two fossil hominoids preserving undistorted femora and pelves from the same individuals and, more importantly, both species have distinctly different locomotor reconstructions. Proconsul is widely hypothesized to have been an above-branch quadruped (Le Gros Clark and Leakey 1951; McHenry and Corruccini 
1983; Walker and Pickford 1983; Beard et al. 1986; Ward 1993; Ward et al. 1993; Kelley 1997; Ward 2007), whereas phalangeal and wrist morphology of Rudapithecus has been related to suspensory behaviors (Begun 1992; 1993; Kivell and Begun 2009; Begun and Kordos 2011; Begun et al. 2012). The results presented in Chapter 5 are consistent with the hypothesized locomotor behaviors used by both Proconsul and Rudapithecus, and suggest that Proconsul would probably not have been capable of the range of abducted hip postures that Rudapithecus could use. This study provides the first evidence for suspensory behaviors based on hindlimb joint mobility in a Miocene ape, and provides important data about hindlimb abilities which are critical for locomotor reconstructions in Miocene apes. 


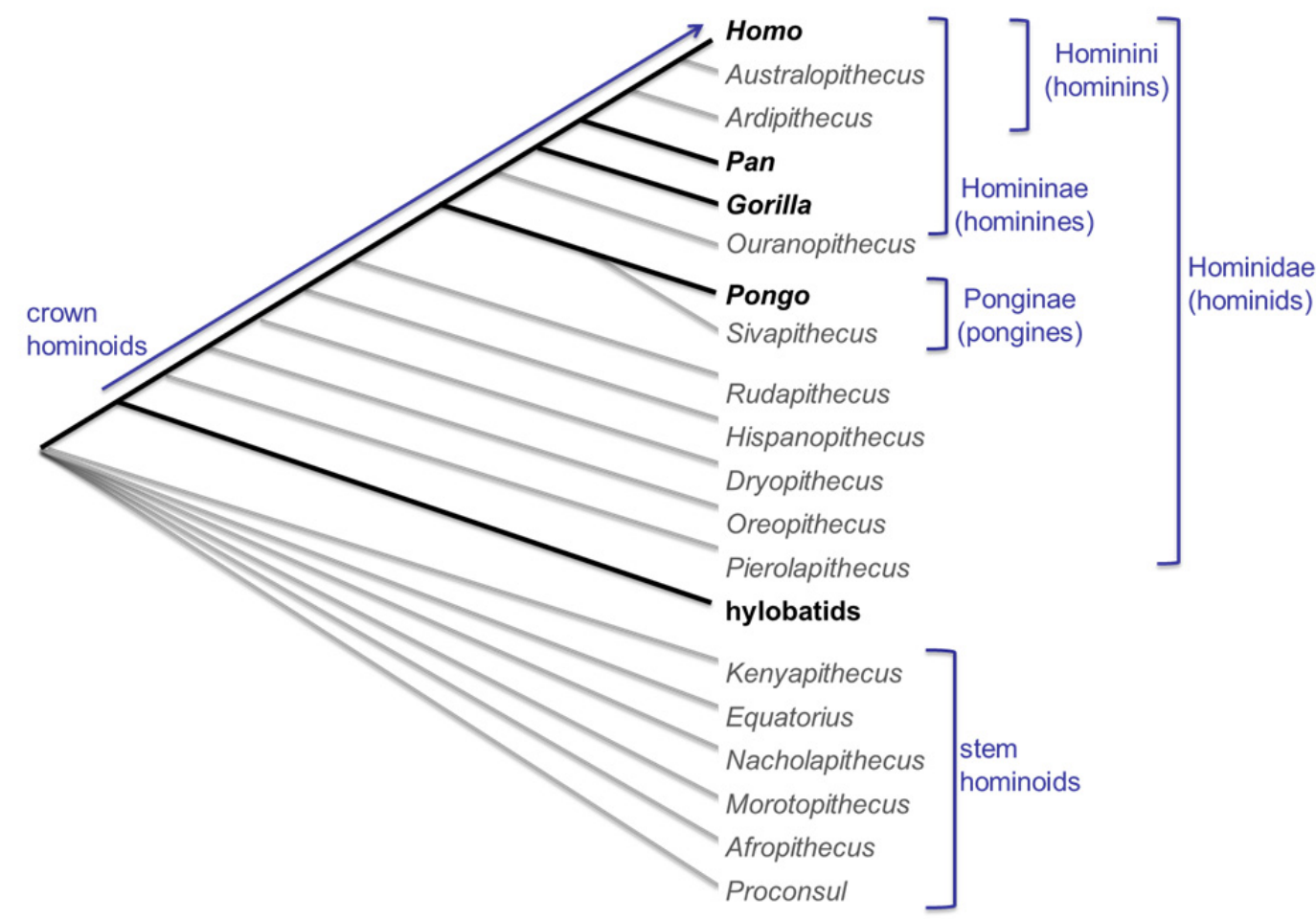

Figure 1.1. Superfamily Hominoidea with hypothesized positions of fossil apes Figure adapted from Crompton et al. (2008), with terminology following Wood and Harrison (2011). Most work supports a distinct group of stem hominoids (Begun et al. 1997a; Finarelli and Clyde 2004; Young and MacLatchy 2004), although the relationships of the fossil hominids is still highly debated among most authors. 


\section{CHAPTER 2: BACKGROUND}

This chapter summarizes previous works that detail anthropoid locomotor behaviors, hip joint soft tissues, and bony morphologies. Locomotor reconstructions for Miocene apes are reviewed within the context of existing hypotheses for locomotor behaviors in Miocene apes. The difficulties in inferring locomotor behaviors in Miocene apes are discussed and followed-up with a discussion of the analytic approach advocated here-- assessing locomotor capabilities in Miocene apes using an in vivovalidated hip joint modeling approach.

\section{RANGE OF MOTION AND ADAPTATION}

Forelimb-dominated arboreality, such as that used during anti-pronograde bridging, brachiation, and vertical climbing, distinguishes hominoids from nearly all cercopithecids, as well as from most anthropoids in general (Keith 1923; Avis 1962; Erikson 1963; Napier 1963). Suspension is a key component to forelimb-dominant locomotor behaviors and is a particularly important adaptation for larger-bodied species, conferring more positional stability by allowing the animals to distribute their weight among multiple arboreal supports rather than balancing over a single support (Grand 1972; Fleagle and Mittermeier 1980) (Figure 2.2). Even though suspensory behaviors only account for a relatively small percentage of locomotor behaviors in the great apes (see Table 2.1), the importance of suspensory behaviors is thought to explain the derived morphologies shared among suspensory anthropoids, such as large intermembral indices and relatively large shoulder breadth (review in Larson 1998). The hypothesis that arboreal versatility is associated with an increased range of hip joint mobility has influenced 
functional inferences made about hip morphology when reconstructing the behavior of fossil primates (Walker 1974; e.g., Fleagle 1983; Rose 1983; Ruff 1988; Ward 1993; Ward et al. 1993; MacLatchy 1996; MacLatchy and Bossert 1996; Köhler et al. 2002; Hogervorst et al. 2009).

Suspensory behaviors have different biomechanical requirements of both the foreand hindlimbs than does generalized quadrupedalism (Grand 1972; Nakatsukasa and Kunimatsu 2009). In particular, suspensory behaviors require reaching for discontinuous, variably-oriented arboreal supports with both the hands and feet (Nakatsukasa and Kunimatsu 2009) (Figure 2.2). Large body size is often cited as being integral to below-branch locomotion because large-bodied animals are more stable when moving below-branch rather than on top of a similarly-sized branch (Fleagle and Mittermeier 1980; Cartmill 1985; Sarmiento 1995; Almécija et al. 2007; Ward 2007), but it is also worth noting that large body size itself facilitates below-branch locomotion. Large body size, particularly when distributed in the forelimbs, increases momentum during below-branch swinging (Demes and Gunther 1989). Additionally, with increasing limb length the physical reach of the animal increases relative to the center of mass, which opens up a greater range of arboreal supports within the animal's reach (Figure 2.1). The concept of the spatial "envelope" (e.g., Stevens and Parrish 1999), whereby the range of distal segment positions show the potential for overall abilities, has been considered in a limited capacity within primates (Grand 1972) but is of extreme importance for understanding locomotor adaptation and overall abilities.

Range of hand and foot positions are influenced by angular excursions at the proximal shoulder and hip joints, making mobility at these joints important determinants of the range of supports within reach of the hand and foot. In particular, increased 
abduction ability at the shoulder and hip joint are attributed to the non-stereotyped reaching movements used during climbing, suspensory, and/or less cursorial behaviors (Jenkins 1974; Walker 1974; Fleagle 1976b; Jenkins and Camazine 1977; Grand 1984; Cartmill 1985; Larson 1993; Ward 1993; 2007; Crompton et al. 2008; Nakatsukasa and Kunimatsu 2009; Schmidt and Krause 2011). Attributions of higher abduction ability in certain locomotor groups are not informed by quantitative data, however, and instead result from qualitative observations regarding joint morphology or limb usage and/or position.

\section{EXTANT ANTHROPOID LOCOMOTOR BEHAVIORS}

Reconstructing the evolution of suspensory behaviors in fossil apes requires identifying morphological functional signals for suspension in extant taxa. Finding clear functional signals is challenging because anthropoids vary in their reliance on suspensory behaviors, and even suspensory apes and atelines, which are highly derived for forelimb-dominated behaviors, vary in their frequency and agility of suspensory behaviors. A large obstacle to all workers interested in anthropoid locomotor behaviors is that it is extraordinarily difficult to classify primates into precise locomotor categories because of their versatility(Rose 1983). All locomotor classifications and descriptive terminology are considerable simplifications of diverse behaviors exhibited by anthropoids but are necessary to test hypotheses regarding functional differences among taxa.

A summary of locomotor behaviors is provided below for the extant taxa considered in this study, and quantitative behavioral observations are provided in Table 2.1 for available taxa. All descriptive terminology for locomotor behaviors follow 
definitions provided by Thorpe and Crompton (2006), Gebo (1992), Cant (1987), and Rose (1973b).

\section{Gibbons (Hylobates) and Siamangs (Symphalangus)}

The hylobatids inhabit dense tropical and subtropical forests in Southeast Asia and travel primarily via below-branch brachiation (reviews in Napier 1967; Napier and Napier 1967; Hunt 1991). White-handed gibbons (Hylobates lar) are very versatile in their locomotor abilities and can move through the canopy via “...diving, leaping, bridging, bipedal walking, running, quadrumanous climbing, and scrambling (Vereecke et al. 2006)." Siamang (Symphalangus syndactylus) locomotion is primarily brachiation along large boughs, supplemented by climbing, leaping, and bipedalism (Fleagle 1976a) (see Table 2.1). Siamangs, larger than most gibbons, are capable of gibbon-like ricochetal brachiation and acrobatic behaviors but exhibit these behaviors with less frequency, for shorter periods of time, and across shorter distances than gibbons (Avis 1962; Chivers 1972; Stern and Oxnard 1973; Fleagle 1976a). There are no known sex differences in locomotor behaviors in gibbons or siamangs.

\section{Orangutans (Pongo pygmaeus, Pongo abelii)}

Orangutans are native to dense rainforests of Indonesia and Malaysia, but are currently restricted to areas of Sumatra and Borneo. There are no major differences known to exist between Sumatran (Pongo abelii) and Bornean (Pongo pygmaeus) orangutan locomotor repertoires, although there may be differences in frequencies of behaviors used between these two species (Cant 1987; Thorpe and Crompton 2006; Manduell et al. 2011). Orangutans use virtually all forms of arboreal travel, including quadrupedal or tripedal walking, vertical climbing, bipedal walking, orthograde (torso 
upright) suspensory clambering, and brachiation (Cant 1987; Thorpe and Crompton 2006) (Table 2.1, Figure 2.3). With the exception of brachiation, all forms of locomotion used by orangutans are slow and cautious-with three of the four extremities typically grasping a substrate at any given time (Davenport 1967). Females tend to be even more cautious in their selection of support types, preferring supports lower in the canopy and larger in diameter (Thorpe and Crompton 2006; Thorpe et al. 2007a; Thorpe et al. 2009).

\section{Western lowland gorillas (Gorilla gorilla gorilla) and Mountain gorillas (G. beringei} $\underline{\text { beringei) }}$

Gorillas inhabit tropical and subtropical forests, swamps, and marshes of Africa. Large interspecific locomotor differences exist between lowland gorillas and the highaltitude mountain gorillas. These locomotor differences are a function of habitat differences, particularly in canopy continuity and availability of foods, which necessitate divergent foraging strategies (Yamagiwa et al. 1998; Goldsmith 1999; Tocheri et al. 2011). Within gorillas, the western lowland gorilla (G. g. gorilla) is the most arboreal throughout its ontogeny and the mountain gorilla (G. b. beringei) is the least arboreal (Doran and Hunt 1994; Remis 1995; Doran 1997; Remis 1998). The western lowland gorilla uses vertical climbing for up to $48 \%$ of arboreal locomotion (Remis 1995) (Table 2.1). There is evidence that gorilla social structure and body size influence locomotor behaviors, with males less likely to use smaller supports and/or suspensory positions than females (Remis 1995). The adult mountain gorilla is almost exclusively terrestrial, although it will use cautious climbing (Schaller 1963; Reynolds 1965; Doran 1996; Doran and McNeilage 1998). Brachiation, swinging, leaping, and prolonged hanging are not observed in the adult mountain gorilla (Reynolds 1965; Doran 1997). 


\section{Chimpanzees (Pan troglodytes) and Bonobos (Pan paniscus)}

Chimpanzees (Pan troglodytes) inhabit forests and savannahs of West and Central Africa whereas the bonobo (Pan paniscus) is found exclusively in Central African forests. Chimpanzee locomotion is dominated by arboreal vertical climbing and terrestrial knuckle-walking (Susman et al. 1980; Doran 1993a; Thorpe and Crompton 2006) (Table 2.1). Male chimpanzees use more arboreal climbing and scrambling and less arboreal quadrupedalism, but are slightly less arboreal overall compared to females (Doran 1993b). The locomotor behavior of bonobos is similar to the chimpanzee, although bonobos are more arboreal than chimpanzees (Doran 1993a). Some studies suggest that bonobos engage in more arm-swinging (brachiation), leaping, diving, and bipedalism when compared to the chimpanzee (Susman et al. 1980; Doran 1993a), with hand and foot postures of captive climbing bonobos described as more variable than other African apes (D'Aout et al. 2004; Isler 2005). The two taxa may also differ subtly in substrate preference during vertical climbing, with chimpanzees preferring largediameter tree trunks and bonobos more frequently using smaller boughs and branches (Doran 1993a).

\section{Colobines (Subfamily Colobinae)}

Colobine monkeys are best described as arboreal quadrupeds (Napier 1967; Napier and Napier 1967). Six colobine taxa are considered within this study, and five of these taxa have few known differences in locomotor behavior. The black and white colobus (Colobus guereza) and western red colobus (Piliocolobus badius ${ }^{1}$ ) are moderately-sized arboreal quadrupeds that will often cover large linear distances by

\footnotetext{
${ }^{1}$ Procolobus badius is a junior synonym of Piliocolobus badius, and both names are frequently used in colobine literature.
} 
leaping (Rose 1973b; Morbeck 1977; Rose 1979; Gebo and Chapman 1995; Struhsaker 2010). Both these species of colobus monkey live in African forests and savannah woodlands. The golden snub-nosed monkey (Rhinopithecus roxellana) lives in montane, temperate forests of southern China and is a highly arboreal species, spending $>97 \%$ of their time in the trees (Li 2007). The golden snub-nosed monkey will use leaping, jumping, and only very rarely semibrachiation (Wu 1993). Proboscis monkeys (Nasalis larvatus) monkeys are large-bodied arboreal quadrupeds, found in mangrove swamps of Malaysia and Borneo, that are reported to leap and dive as frequently as smaller colobines (Kern 1964; 1965; Rose 1973b). Lutungs (e.g., Trachypithecus cristatus) are small agile arboreal quadrupeds that also frequently leap and climb (Furuya 1961; Fleagle 1998; Harding 2010). Lutungs inhabit a range of habitats in Southeast Asia but prefer rainforests.

Within the Old World Monkeys, doucs (Pygathrix) are the only group reported to use below-branch suspensory behaviors (Workman and Covert 2005; Wright et al. 2008), although the majority of reports are based on captive primates. Doucs are entirely arboreal (Lippold 1998) and live in rainforests on mainland Southeast Asia. Captive doucs travel via brachiation or suspension as often as they travel quadrupedally, with pulling-up, climbing and/or leaping comprising only a small percentage of behaviors (Wright et al. 2008). Until doucs are more extensively studied, it is difficult to identify how important below-branch behaviors are in this group.

\section{Cercopithecines (Subfamily Cercopithecinae)}

Three cercopithecine taxa, patas monkeys (Erythrocebus patas), baboons (Papio sp.), and geladas (Theropithecus gelada), are highly terrestrial (Rose 1973b; a; 1977; Hunt 1991; Fleagle 1998) and typically only use arboreal behaviors for safety from 
predators (Enstam and Isbell 2004). These species inhabit open habitats and grasslands with limited opportunities to use arboreal substrates, and are particularly limited in opportunities to leap between trees, which are distantly spaced (Hall 1966; Rose 1973b; Isbell et al. 1998). Terrestrial quadrupedalism accounts for $97 \%$ of all locomotion in baboons (Papio) (Rose 1977; Hunt 1991). The time geladas spend moving each day in search of food is the highest of any primate, although the distance traveled per day may be less than some baboon species (Iwamoto 1993). No documentation of suspensory locomotion or posturing could be found for wild baboons, geladas, or patas monkeys.

Although some cercopithecines are somewhat terrestrial, many cercopithecines use arboreal behaviors in high frequencies. The blue monkey (Cercopithecus mitis) is primarily an arboreal quadruped, whose dominant forms of locomotion are quadrupedalism and climbing, with suspensory behaviors (e.g., bridging, bimanualism) rarely observed (Gebo and Chapman 1995).

Macaques are quadrupedal monkeys, although there are interspecific differences in arboreal proclivities. Crab-eating macaques (Macaca fascicularis) are primarily arboreal quadrupeds (Rodman 1979; Cannon and Leighton 1994). Other modes of travel include scrambling (15\%), climbing (12\%), and leaping (6.4\%) (Cannon and Leighton 1994). The pigtail macaque (Macaca nemestrina) is substantially more terrestrial than the crab-eating macaque, preferring to return to the ground to travel between tree crowns rather than leaping (Rodman 1979).

\section{Spider monkeys (Ateles)}

Spider monkeys (Ateles sp.) live in tropical forests and rainforests of Central and South America. Spider monkeys are the most agile and suspensory of the New World 
monkeys (Erikson 1963; Eisenberg and Kuehn 1966; Napier and Napier 1967; Campbell 2008). Locomotor behaviors frequently include most forms of arboreal locomotion, including forelimb, hindlimb and/or tail-assisted suspensory behaviors, quadrupedalism on large-diameter supports, leaping to cross discontinuous gaps, and climbing (Mittermeier and Fleagle 1976; Mittermeier 1978; Cant 1986; Cant et al. 2001; Youlatos 2002). Quantitative data from Mittermeier (1978) shows that suspensory behaviors and climbing behaviors are preferentially used over quadrupedal behaviors in both Ateles paniscus (suspension 39\%, climbing 31\%, quadrupedalism 25\% ) and Ateles geoffroyi (suspension 26\%, climbing 40\%, quadrupedalism 22\%).

\section{Howler monkeys (Alouatta)}

The howler monkey (Alouatta caraya) lives in similar New World environments as the spider monkey, but, whereas the spider monkey is highly suspensory, the howler monkey moves by slow quadrupedal progression along relatively large horizontal arboreal supports (Grand 1968; Mendel 1976; Fleagle and Mittermeier 1980). The deliberate, non-acrobatic quadrupedalism of the howler is potentially derived within atelids (Rosenberger and Strier 1989; Jones 2008). Howlers are reported to climb frequently when on smaller-diameter supports (Fleagle and Mittermeier 1980; Gebo 1992; Bicca-Marques and Calegaro-Marques 1995). Arm-swinging and leaping behaviors are only rarely observed (Grand 1968; Fleagle and Mittermeier 1980; Ybarra 1984; Gebo 1992), although quadrumanous bridging behaviors are frequent (Prates and Bicca-Marques 2008). The howler monkey stabilizes itself with its prehensile tail (Lawler and Stamps 2002). 


\section{Capuchin monkeys (Cebus sp.)}

Capuchin monkeys (Cebus sp.) are New World monkeys that can inhabit tropical, subtropical, and dry forests, as well as sometimes more open, disturbed habitats. Capuchins are arboreal quadrupeds and occasional leapers (Fleagle and Mittermeier 1980; Fleagle et al. 1981a; Gebo 1992; Wright 2007). The small body size of capuchins allows these animals to leap more frequently than the larger atelids (Fleagle and Mittermeier 1980).

\section{$\underline{\text { Locomotor adaptation }}$}

Adaptive traits have a current functional role in an organism and are maintained in the population by means of natural selection. A species adapted for suspensory behaviors will have their survival and reproductive success increased by suspensory behaviors. Testing the importance of suspensory behaviors for an organism's survival and reproduction is virtually impossible, however. It is possible to measure an organism's frequency of suspension and qualitatively assess the agility and willingness to perform suspensory behaviors, and, although not ideal, functional morphologists interested in suspensory behaviors often base inferences about suspensory locomotor adaptation from frequency data.

It is difficult to classify extant primates into distinct locomotor groups (Erikson 1963; Napier 1967; Napier and Napier 1967) and, in fact, none of the extant primates can be considered exclusively "suspensory" or "non-suspensory". Most primates are probably capable of using suspensory behaviors. Locomotor classifications are further complicated by the fact that even within the more strictly suspensory taxa there is a spectrum of reliance on suspensory behaviors, with hylobatids using suspension for at least 50\% of locomotion (Napier 1963; Napier and Napier 1967; Fleagle 1976a; Hunt 
2004) and species such as chimpanzees using pure suspension for only $7 \%$ of locomotor bouts (Doran 1996).

The published behavioral literature has been used to classify the extant taxa considered in this study into very broad categories based on reliance on suspension (Table 2.2, Figure 2.4). Non-suspensory taxa are those that never use suspension when moving by pronograde movement is an alternative, and includes generalized arboreal and terrestrial quadrupeds such as capuchins (Cebus) and most cercopithecoids (Napier and Napier 1967; Rose 1973b; a; Morbeck 1977; Rose 1977; 1979; Fleagle and Mittermeier 1980; Fleagle et al. 1981a; Gebo and Chapman 1995; Struhsaker 2010). Species classified as suspensory include the hylobatids (Hylobates, Symphalangus), orangutans (Pongo sp.), lowland gorillas (Gorilla gorilla), chimpanzees (Pan troglodytes), bonobos (Pan paniscus), and spider monkeys (Ateles sp.). Hylobatids and orangutans prefer to move via below-branch locomotion, and are able to negotiate almost all arboreal substrate sizes, orientations, and continuities with ease (Davenport 1967; Chivers 1972; Fleagle 1974; Fleagle 1976a; Cant 1987; Thorpe and Crompton 2006; Manduell et al. 2011). Chimpanzees, bonobos, western lowland gorillas, and spider monkeys use less suspension but it is still an important part of their locomotor repertoire (see Table 2.2) (Susman et al. 1980; Cant 1986; Doran 1993a; Doran and Hunt 1994; Remis 1995; Doran 1997; Remis 1998; Cant et al. 2001; Youlatos 2002).

Additionally, there are taxa that are difficult to classify into suspensory or nonsuspensory categories and have been excluded from categorization in this study. As previously discussed, howler monkeys (Alouatta) and mountain gorillas (G. beringei) are generally quadrupedal but probably still retain some suspensory adaptations (Schaller 1963; Reynolds 1965; Fleagle and Mittermeier 1980; Bicca-Marques and Calegaro- 
Marques 1995; Doran 1996; Doran and McNeilage 1998). Doucs (Pygathrix) seem to use brachiation quite frequently in captivity but it is unclear how substantial an adaptation this might be in wild-populations (Workman and Covert 2005; Wright et al. 2008). Data for Pygathrix, Alouatta, and G. beringei are presented in this dissertation, in the hope that future behavioral work will more clearly determine whether these taxa can be assigned to a locomotor category.

\section{EXTANT ANTHROPOID HIP JOINT RANGE OF MOTION}

Behavioral data suggests that that below-branch behaviors require high joint mobility, particularly abduction, in the shoulder (Le Gros Clark 1959; Grand 1972; Rose 1973b; Jenkins 1974; Grand 1984; Larson 1993; Hunt 2004; Crompton et al. 2008) and hip joints (Cartmill 1985; MacLatchy 1996) to enable reaching for distantly spaced arboreal supports, although very little quantitative data exist. Some inferences of joint motion can be gathered from 3D kinematic studies (e.g., Isler 2005) but the majority of kinematic studies are 2D and incapable of measuring anything other than flexionextension angles. Isler (2005) measured hip abduction during vertical climbing in a small sample of captive hominoids, finding abduction to be similar across the great apes during vertical climbing tasks and slightly reduced in hylobatids. The thigh achieved maximal abduction in great apes during more flexed postures. Unfortunately, these are the only known data on hip abduction in nonhuman anthropoids and, problematically, laboratory and zoo kinematic data probably do not represent the extreme, or possibly even the typical, ranges of motion that are elicited in wild environments (Vereecke et al. 2011).

Some data exist for passive joint mobility, which is the best metric of overall range of motion but has limited inferential utility regarding specific ranges of joint motion 
used during different locomotor behaviors. DeRousseau et al. (1983) report data for passive hip joint abduction in free-ranging Macaca mulatta $(n=126)$, representing the only known passive hip joint abduction data in nonhuman anthropoids. The authors report no significant differences between right and left hip joints or age, and but some differences between sexes (see Table 2.3). Two other studies have examined range of passive hip joint motion in non-human anthropoids, although these studies focused on range of flexion-extension and did not quantify abduction. A large cross-sectional study by Turnquist and Kessler (1989) examined joint mobility in free-ranging Macaca mulatta ( $n=661$ ), finding that range of hip flexion-extension decreased with age. A related study examined caged $(n=85)$ and free-ranging $(n=64)$ Erythrocebus patas, finding that joint mobility in caged animals can be highly variable (Turnquist 1983), with the largest differences in mobility attributed to animals raised in cage confinement from a young age (Turnquist 1983). Hip extension was the only hip variable measured but was found to be particularly susceptible to reductions in mobility due to caging compared to joints such as the wrist, ankle, and shoulder (Turnquist, 1983).

There is a large amount of data available for human hip joint mobility (see Table 2.3). Professional ballet dancers, who elicit extreme hindlimb postures, have similar ranges of passive hip joint motion as the general population, but have significantly increased ranges of flexion, abduction, and external rotation (Charbonnier et al. 2011). The large amount of human data shows that there is minimal difference between goniometric and more technologically advanced (e.g., Vicom) range of motion measurement techniques (American Academy of Orthopedic Surgeons 1965; Cole 1971; Kendall et al. 1971; Esch and Lepley 1974; Hoppenfeld 1976; Cailliet 1978; Boone and Azen 1979; Roaas and Andersson 1982; Mohr 1989; Pedretti 1996; Steinberg et al. 
2006; Kubiak-Langer et al. 2007; Charbonnier et al. 2011; Kumar et al. 2011; Moreside and McGill 2011), indicating either approach will converge on similar values.

Unfortunately, most work examining hip mobility and limb postures has been restricted to a single taxon or clade, limiting the ability to extend conclusions about joint function to locomotor adaptation. Because it is untested whether hip mobility actually differs between species with different locomotor adaptations, it is unknown whether hip function actually reflects behavioral adaptation. This has been highlighted by recent work on the shoulder joint (Chan 2007b; a; 2008), which tested the assumption that suspensory primates have an increased range of motion at the shoulder joint to facilitate below-branch behaviors and whether thoracic shape actually promotes different levels of shoulder mobility. Chan's work found that the hominoid shoulder joint does not actually produce a greater range of circumduction than other primates (Chan 2007a), which would imply that non-suspensory monkeys have high shoulder mobility but do not routinely use locomotor behaviors which elicit the arm postures used by suspensory species. This scenario is difficult to reconcile with the different glenohumeral morphologies observed between apes and non-suspensory monkeys, and underscores the importance of comparing models of joint motion with empirical data.

\section{INFLUENCE OF SOFT TISSUE MORPHOLOGY ON HIP MOBILITY}

This study aims to reconstruct range of motion in fossil taxa using just bony morphology, making it necessary to consider the role of soft tissues in limiting hip mobility. Since range of hip motion is primarily determined by ligament, tendon and passive muscle tension (Kapandji 1970; Wright and Radin 1993; Levangie and Norkin 2005; Safran et al. 2012), there could be a considerable loss of information in models that do not include soft tissues (see Hutson and Hutson 2012). It is possible to build 
models from living animals with intact soft tissues (Brainerd et al. 2010; Gatesy et al. 2010) or to incorporate information about muscle dimensions and attachments into simulations of joint motion (Ogihara et al. 2009; Seth et al. 2011; O'Neill et al. 2013). However, Incorporating soft tissue constraints into models of extinct species includes substatial guesswork regarding ligament and muscle fiber orientation, size, tension, etc. (Wang et al. 2004; Hutchinson et al. 2005; Nagano et al. 2005), with each additional parameter adding another level of uncertainly (and potentially error) to the model (Hutchinson and Garcia 2002; Hutchinson 2004; 2011). In order to facilitate discussions of why the models deviate from empirical range of motion data when soft tissues are not present, the soft tissue structures of the anthropoid hip joint must be considered.

\section{$\underline{\text { Hip joint capsule and capsular ligaments }}$}

The joint capsule, particularly in reference to the three capsular ligaments, is one of the strongest soft tissue limits on femoral head movement within the acetabulum (Myers et al. 2011; Smith et al. 2011; Smith et al. 2012). The capsule surrounds most of the neck of the femur, attaching along the intertrochanteric line anteriorly and at the base of the neck posteriorly, and along the border of the acetabular labrum on the pelvis. The hip joint capsule physically limits femoral head movements via the capsular ligaments, keeps the femoral head tight in the acetabulum by creating negative intra-articular pressure, provides information about joint posture due to its high number of proprioceptive nerve endings, and even provides acute sensory (e.g., pain) information about joint position (Ralphs and Benjamin 1994; Levangie and Norkin 2005).

The three capsular ligaments are the iliofemoral (Y-ligament of Bigelow), ischiofemoral, and pubofemoral. The human ischiofemoral, pubofemoral, and the two limbs of the iliofemoral ligament, each have discrete functions to resist femoral head 
translation as the hip moves through its range of motion (Martin et al. 2008; Smith et al. 2012). Within humans, the iliofemoral ligament attaches along the anterior inferior iliac spine and iliac acetabular margin, with the superior band attaching at the proximal intertrochanteric line and base of the greater trochanter and the inferior band attaching along the distal intertrochanteric line (Martin et al. 2008; Wagner et al. 2012). The iliofemoral is the strongest of the ligaments, and functions to resist extension and external rotation in humans (Myers et al. 2011), with the superior band primarily responsible for limiting external rotation and the inferior band limiting extension (Wagner et al. 2012). The ischiofemoral ligament attaches along the caudal acetabular border and the superior aspect of the femoral neck and intertrochanteric line, limiting internal rotation (Wagner et al. 2012). The pubofemoral ligament originates along the obturator crest and superior pubic ramus and blends with the iliofemoral ligament distally to insert near the lesser trochanter, acting to limit abduction (Wagner et al. 2012).

Little information is available on the hip joint capsule in primates. The capsule in the rhesus macaque is strong, with a broad, thick iliofemoral ligament (Howell and Straus 1933). The orangutan joint capsule is lax with capsular ligaments that are not distinct (Sonntag 1924). The chimpanzee joint capsule lacks all ligaments except one band of the iliofemoral (Sonntag 1923). These limited data suggest that the great ape capsule is looser and allows more mobility than that of cercopithecids.

\section{Ligamentum teres femoris}

The human ligamentum teres femoris primarily arises from the acetabular transverse ligament, but also arises from the cranial and caudal margins of the acetabular notch (Cerezal et al. 2010). The ligament is fully intracapsular, with an external synovial layer bounding the dense, well-organized collagen fibers that compose 
the ligament proper (Chen et al. 1996). It is normally $30-35 \mathrm{~mm}$ long in humans (Chen et al. 1996; Bardakos and Villar 2009). In most mammals, the ligamentum teres transmits the artery of ligamentum teres, a branch of the obturator artery, to the capital femoral epiphysis during growth.

After the artery of ligamentum teres closes in adulthood, it is unclear what functional role of the ligamentum teres retains. Several studies have found a link between adult joint stability and ligamentum teres integrity, although whether the intraarticular ligament confers joint stability via proprioceptive feedback (Leunig et al. 2000; Sarban et al. 2007) or biomechanically limiting joint motion (Chen et al. 1996; Demange et al. 2007; Wenger et al. 2007; Dodds et al. 2008; Bardakos and Villar 2009; Martin et al. 2011) is uncertain. If the ligamentum teres is mechanically limiting joint movements, it appears to do so when the femur is in a position of combined adduction, flexion, and external rotation (Dodds et al. 2008).

The ligamentum teres configuration has become a central point in discussions of hip joint mobility and locomotor behavior (Walker 1974; Crelin 1988; MacLatchy 1995; 1996; MacLatchy and Bossert 1996; Harmon 2007), largely because the habitual use of joint positions that impinge the ligamentum teres between the femoral and lunate articular surfaces could potentially lead to cartilage degradation, osteoarthritis, and traumatic joint injury (Kapandji 1970; Jenkins and Camazine 1977; Mow et al. 1989; Ward et al. 1993; Notzli et al. 2001). The orangutan, a large-bodied and long-lived species that employs extreme hip joint postures, has a ligamentum teres configuration which theoretically minimizes the potential for degenerative hip joint diseases by positioning the ligamentum teres away from the joint loading surface. Secondary support for this being an adaptation related to minimizing joint degradation comes from 
the "sloth-like" subfossil paleopropithecids, which also lack a ligamentum teres insertion on the femoral head and are hypothesized to have been completely suspensory (Godfrey et al. 2010). The orangutan ligamentum teres insertion has been called unique among anthropoids because it occurs at the base of the posterior medial articular surface of the proximal femur, on the femoral neck rather than in the center of subchondral bone (Crelin 1988), essentially removing it from the joint loading surface. Indian elephants also lack a subchondral ligamentum teres insertion (Crelin 1988), so this morphology does not exclusively relate to joint mobility and arboreal versatility, and it is emphasized here that a repositioned ligamentum teres insertion probably relates to maximizing joint loading surface to minimize potential for joint degradation. Although the unusual ligamentum teres insertion in orangutans has received considerable attention, little information on ligamentum teres structure has been published in other nonhuman primates.

\section{Labrum}

The acetabular labrum, a fibrocartilaginous ring around the border of the acetabulum, constrains movements of the femoral head within the acetabulum by deepening the acetabular socket and by increasing the negative pressure of the joint (Crawford et al. 2007; Myers et al. 2011; Smith et al. 2011; Safran et al. 2012). A larger acetabular labrum is implicated in certain types of femoroacetabular impingement in humans, with patients having a decrease in joint mobility associated with labral overcoverage of the femoral head (Tannast et al. 2007). No data are currently available on labrum size in nonhuman anthropoids. 


\section{Muscles}

Muscle-tendon units can contribute to differences in mobility observed between species, with movements probably limited by differences in muscle extensibility and length (Gajdosik 2001; Gerling and Brown 2013). Thick muscles spanning the joint could also hypothetically limit motion at joints by physically obstructing movements at the joint, although this has not been reported in clinical literature. Most anthropoids have muscle insertion and origin patterns that broadly correspond to humans but the muscles attach on bones that may differ markedly in shape between taxa, which could result in different muscular orientations across joints. Moreover, it is known that some apes have muscle-chains (e.g., muscles that insert on other muscles) which span joints (Jungers and Stern 1980), although this is entirely unexplored in terms of how this might affect joint mobility.

The gluteus maximus muscle in apes (excluding orangutans) has 2 functional portions (Sigmon 1974). The proximal portion of the gluteus maximus inserts on the iliotibial tract and functions to abducts, laterally rotates, and tenses the iliotibial tract (Sonntag 1924; Sigmon 1974). The distal portion inserts from the gluteal tuberosity distally along the femur, and functions to extend, adduct, and laterally rotate the thigh (Sonntag 1924; Sigmon 1974). The gluteus maximus in cercopithecines is considered a single functional unit, although associated slips of muscle traveling from the base of the tail to the femur are sometimes attributed to caudofemoralis or femorcoccygeus as in other quadrupeds (Howell and Straus 1933). It should be noted that the reorientation of the iliac blades in humans results in a drastically different gluteus maximus action (primarily hip extension) than is observed in quadrupeds such as cercopithecoids. 
Only the cranial portion of the gluteus maximus is present in orangutans, which has a functionally similar yet distinct muscle called the ischiofemoralis muscle in place of the distal (caudal) portion. The orangutan ischiofemoralis originates in common with the long head of the biceps femoris muscle and inserts along the proximolateral border of the femoral shaft, almost to the lateral epicondyle. The actions of the ischiofemoralis muscle include extension, lateral rotation, and adduction of the femur. Additionally, the orangutan gluteal region can be further differentiated from other primates in that the tensor fascia lata muscle is absent (Sigmon 1974).

The iliotrochantericus muscle is a small variably present slip muscle that assists in hip flexion (Raven 1950; Satoh 1965; Sigmon 1974). It arises deep to the origin of the rectus femoris and inserts on the lesser trochanter, inferior to the iliopsoas insertion (Sigmon 1974). However, it should be noted that iliotrochantericus will often arise from the hip joint capsule itself (Raven 1950; Satoh 1965).

The adductor magnus, a single muscle with adductor and hamstring portions in humans, generally presents as two distinct portions in nonhuman anthropoids. The hamstring portion, or ischial portion, is called the long head, with the short head being the portion that attaches on the pubis (Preuschoft 1961; Sigmon 1974). Although adductor magnus is described in terms of two functional portions in platyrrhines, it is not clear if the two functional portions have two distinct heads as in catarrhines (Stern 1971).

Biceps femoris structure is highly variable in primates. In cercopithecids, the biceps femoris consists of just the long head, running from the ischial tuberosity to the tibia and fascia lata (Howell and Straus 1933). Apes and most New World monkeys have the bicipital form (Sonntag 1923; 1924; Raven 1950; Schön 1968; Stern 1971), although occasionally this muscle presents as a single head in hylobatids and 
chimpanzees (Sigmon 1974). In the extant apes, the long head inserts on the lateral prominence of the tibia, as well as potentially on the iliotibial tract. The long head of the biceps femoris in orangutans may also insert on the femoral shaft (Howell and Straus 1933). The biceps femoris short head arises on the distal femoral shaft and joins the long head to insert on the lateral tibia, or on the head of the fibula and crural fascia (Raven 1950; von Klaus Uhlmann 1968; Sigmon 1974).

The scansorius muscle is consistently found in orangutans (Sonntag 1924; Sigmon 1969) but is variably present in other great apes (Raven 1950; Payne 2001), hylobatids (Diogo 2012), and cercopithecids (Satoh 1965). The scansorius muscle assists in thigh abduction, flexion, and medial rotation (Sigmon 1969; 1974). In particular, the scansorius is thought to aid in drawing the thigh up towards the body in a scanning movement, from a position of abduction to flexion, as might be used in climbing (Sigmon 1969). Scansorius arises from the dorsolateral surface of the iliac blade and inserts anterior to the greater trochanter, just distal to the gluteus minimus insertion (Waterman 1929; von Klaus Uhlmann 1968; Sigmon 1969).

Additional muscles (i.e., iliotrochantericus, scansorius) and additional functional portions of muscles (i.e., gluteus maximus, adductor magnus, biceps femoris) in nonhuman primates likely provide finer control of thigh movements or potentially increase force in certain movements. In terms of maximum (passive) range of motion at the hip, there is no reason to expect that these muscles are positioned in a way that would obstruct joint movement. Detailed dissection and histological work would be required to assess variation in muscle elasticity, architecture, and fiber lengths and how they relate to mobility. However, should there be differences in range of motion between 
locomotor groups in vivo, differences in muscular structure should be explored as a contributing factor.

\section{OSTEOLOGICAL CORRELATES OF SUSPENSION}

Although suspensory behaviors range from high-speed richochetal brachiation in hylobatids, a more cautious climbing and clambering in great apes, and tail-assisted quadrupedal clambering in atelines, there are certain osteological features attributed to suspension across species. Osteological correlates of suspension include a high intermembral index, long curved phalanges, relatively small pollex, broad shoulders and often a broad ribcage, reduced or lost ulnocarpal articulation, laterally-facing glenoid, and a ventral displacement of the vertebral column (Erikson 1963; Tuttle 1975; Cartmill and Milton 1977; Sarmiento 1995; Larson 1998). In great apes, the reduction in the number of lumbar vertebrae, and dorsally displaced transverse processes on the vertebrae are also attributed to orthograde behaviors (Schultz 1950; 1961; Sarmiento 1995).

\section{Features of the femur and pelvis attributed to high hip mobility}

In all anthropoids, the spherical femoral head fits within the cup-shaped acetabular socket and articulates against a raised C-shaped portion of the acetabulum called the lunate surface. The lunate surface and the femoral head (not including the fovea capitis) are covered by articular hyaline cartilage. All movements of the femoral head within the acetabulum are centered at the hip joint center (Kapandji 1970; Pauwel 1980; Levangie and Norkin 2005). Movements are primarily limited by soft tissue constraints, however, as previously outlined. Of particular interest here, the ligamentum teres (and therefore, its insertion on the femur -- the fovea capitis) is necessarily 
confined to the acetabular fossa to avoid risk of potentially damaging peak pressures on articular cartilage during normal joint loading (Kapandji 1970), which can lead to cartilage degeneration (Mow et al. 1989; Notzli et al. 2001). Based on this model of joint movement outlined by (Kapandji 1970) and (Jenkins and Camazine 1977), many bony morphologies of the pelvis and femur are specifically hypothesized to relate to hip mobility and/or suspension (Waterman 1929; Napier and Walker 1967; Schultz 1969; Jenkins 1972; Walker 1974; Jenkins and Camazine 1977; Stern and Susman 1983; Fleagle and Anapol 1992; Ward 1993; Ward et al. 1993; MacLatchy 1995; 1996; MacLatchy and Bossert 1996; MacLatchy 1998). It has thus been interpreted that a correspondingly shallow acetabulum, large acetabular fossa, large femoral head, a centrally-positioned fovea capitis (when present), long and steeply-inclined femoral neck, and a short greater trochanter reflect high abduction abilities (Schultz 1969; Jenkins 1972; Walker 1974; Lovejoy 1975; Jenkins and Camazine 1977; Ruff 1988; Ward et al. 1993; MacLatchy and Bossert 1996) (see Figures 2.5-2.6).

Most workers agree that a fovea capitis that is absent or centrally-positioned on the subchondral surface indicates a mobile hip joint (Walker 1974; Jenkins and Camazine 1977; Crelin 1988; MacLatchy 1995; 1996; MacLatchy and Bossert 1996; MacLatchy et al. 2000; Harmon 2007), although there is not a consensus among authors as to how to interpret articular surface distribution in anthropoids. The articular morphology of the opposing femoral and lunate surfaces should reflect the limits of normal motion at the hip, and therefore also reflect the positions of the joint during routine loading. The extent of the acetabular fossa within the lunate surface reflects maximum normal movements of the femoral head within the acetabulum (Kapandji 1970), but the femoral head articular surface generally receives the most discussion. 
Asfaw (1985) suggests that the articular margin is variable within a single species, with the range of variation observed in humans encompassing what is described as the normal chimpanzee morphology by Jenkins (1972). Other authors argue for broad patterns in femoral head articular configuration that correspond to different locomotor modes or habitual hip postures. For instance, a relatively larger amount of subchondral bone on the anterior aspect of the femoral head has been related to a greater emphasis on adducted hip postures during locomotion (Fleagle 1976b; MacLatchy and Bossert 1996), although a distally-positioned fovea with the articular surface expansive posterosuperiorly has also been attributed to typically adducted, parasagittal limb movements (Walker 1974; Jenkins and Camazine 1977; Fleagle and Meldrum 1988) (Figure 2.6). Fleagle (1976b) relates an expansive anterior articular surface with an articular surface extending onto the femoral neck posteriorly as being related to adducted posture, although it has been observed that chimpanzees typically have a posteriorly expansive articular surface as well (Jenkins 1972; Stern and Susman 1983). Strepsirrhines have a slightly different femoral head articular structure probably related to different, highly-specialized leaping behaviors in this group (Walker 1974; Godfrey 1988; Anemone 1990; Dagosto and Schmid 1996).

A large and spherical femoral head is typically taken as an indicator of high mobility at the hip joint (Fleagle 2013). Ruff (1988) argues that high sphericity of the femoral head allows continued contact between femoral head and the acetabulum. Specifically, a large femoral head relative to the anteroposterior dimension of the femoral neck allows increased joint excursion compared to a relatively smaller femoral head (see Figure 7 in Ruff 1988). Given that Pongo has the most spherical femoral heads (77\% of a sphere), they should have increased potential for joint excursion compared to Pan and 
Gorilla ( 71\%) (Ruff 1988). Of course, joint size is a function of competing demands for mobility and weight-bearing, and a relatively large femoral head is alternatively attributed to either high levels of hip mobility or increased joint loading (Ruff 1988; Harmon 2007). When humans are excluded, hindlimb joint surface area typically scales isometrically across non-human primates (Alexander 1980; Ruff 1988; Godfrey et al. 1991; Jungers 1991). Because of the overlap between highly suspensory species and bipedal species in terms of femoral head proportions and shape (Harmon 2007), it can be challenging to identify locomotor behavior in fossil taxa for which both scenarios are plausible (e.g., Oreopithecus, Orrorin).

Moreover, how the femoral head is hafted onto the femoral neck affects the freedom of the femoral head from the neck, affecting movement within the acetabulum (Hogervorst et al. 2009). The declination of femoral head (e.g., superoinferior orientation of the femoral head articular surface on the neck) and the anteversion of the femoral head (e.g., anteroposterior orientation of the femoral head articular surface on the neck) are related to how the femoral head actually sits within the acetabulum. Orientation of the femoral head is often used interchangeably with articular surface distribution and approached as a non-metric trait (Jenkins 1972; Stern and Susman 1983; Asfaw 1985), and so there are no formalized hypotheses as to how variation in this feature might relate to hip mobility.

Depth of the acetabulum should also affect the maneuverability of the femoral head within the hip socket, and some work on humans suggests that acetabular orientation influences femoral head configuration and movement as well (D'Lima et al. 2000; Levangie and Norkin 2005; Steppacher et al. 2008). The acetabulum in suspensory species is usually described as being somewhat shallow, although there is a 
range of variation, with Pongo being extremely shallow and Gorilla being quite deep by comparison (Schultz 1969; Ward 1991; Ward et al. 1993; Hogervorst et al. 2009). The C-shaped lunate surface, which articulates with the femoral head, displays differences in width of the anterior (cranial) horn and the width of the acetabular notch (Schultz 1969; Ward 1991; Ward et al. 1993). There is limited evidence that Gorilla may also have a reduced amount of articular cartilage present on the anterior horn (i.e., the anterior horn is not fully covered by cartilage in some individuals), which would correspond to an apparently reduced articular surface area distal (inferior) to the fovea capitis (Tillmann 1978).

Although most work has focused on the hip joint articulation, bony geometry of the proximal femur is also cited as an indicator of abduction ability. A long femoral neck will theoretically increase the distance between the bony pelvis and the greater trochanter, allowing for more abduction potential (Fleagle and Meldrum 1988). Likewise, it is hypothesized that a lower greater trochanter should enable more abduction before impingement of bony or soft tissues occurs (MacLatchy 1995; 1996). A tall greater trochanter will also reduce the range of powered abduction because the gluteus medius and minimus will more quickly approach the end of their effective length-tension range (Rodman 1979; Ting 2001). A higher neck-shaft angle could mitigate some of the effects of a tall greater trochanter by positioning the femoral head above the greater trochanter (Ward 1992), especially when coupled with a long femoral neck length (Marivaux et al. 2008).

The function of the crista trochanterica, a tubercle on the posterior aspect of the femoral neck, is unknown but there are hypotheses that the crista trochanterica relates to either an increase or a decrease in hip joint mobility. It has been hypothesized to 
increase the leverage of the obturator internus muscle running over the tubercle, specifically during hip flexion in primates with a short femoral neck (see Alan Walker communication in Napier 1964). In this scenario, the crista trochanterica would confer enhanced external rotational abilities. However, the tubercle is present in many lemurs, platyrrhines, cercopithecoids, and hylobatids (Napier 1964; Rose et al. 1992; MacLatchy et al. 2000; Bacon 2001; Köhler et al. 2002), as well as in early Miocene hominoids (e.g., Equatorius BMNH 16331, Morotopithecus MUZ-M80, Nacholapithecus KNM-BG 35250, Proconsul KNM-RU 1753 and KNM-MW 13142) (Le Gros Clark and Leakey 1951; Napier 1964; Rose et al. 1992; MacLatchy et al. 2000; Nakatsukasa et al. 2012). It is unclear why cercopithecoids would require powerful external rotation and hylobatids do not fit with the short neck model. An alternate hypothesis, favored here, is that the tubercle relates to a stronger or thickened ischiofemoral ligament (Le Gros Clark and Leakey 1951), which would suggest that a large tubercle might reflect reduced joint mobility. Interestingly, the position of the crista trochanterica differs between extant taxa, with Ateles displaying a superior position and Papio being positioned more inferiorly on the posterior femoral neck (Bacon 2001). When found in Miocene femora, the crista trochanterica is described as being superiorly positioned (Bacon 2001; Nakatsukasa et al. 2012), perhaps reflecting hip joint mobility more similar to Ateles.

\section{Features of the femur and pelvis that increase span at the foot}

Ultimately, hip mobility is important for increasing the spatial envelope, or the range of positions that the distal limb segments can occupy (Figure 2.1). However, positions of the knee, and ultimately the foot, are also determined by factors other than mobility. Most notably, this includes body size. Absolutely and relatively larger femoral diaphysis lengths, femoral neck lengths, and bi-acetabular breadths can all facilitate 
positions of the distal limb segment that are further from the midline of the body simply because they are larger. A large neck-shaft angle increases the abduction and lateral position of the knee for a given position of the hip (Walker 1974; Fleagle 1977; Jenkins and Camazine 1977; Rose 1983; Harrison 1986; Fleagle and Meldrum 1988), accomplishing the opposite of a large bicondylar angle (see Figure 2 in Jenkins 1972).

\section{HOMINOID EVOLUTION}

\section{Fossil evidence of locomotor evolution in Miocene apes}

Hominoids diverged from other catarrhines more than 20 Ma (review in Stewart and Disotell 1998), and probably closer to 25 Ma based on a few recently discovered fossils (Stevens et al. 2013). Most stem catarrhines, except perhaps Apidium (Fleagle and Simons 1995), share a large number of postcranial similarities with the later Miocene hominoids (review in Simons 1995; femur specific morphology in Ankel-Simons et al. 1998). A few authors suggest that early hominoid Proconsul may be a stem catarrhine (Harrison 1987; 1993; Rossie et al. 2002), but this thesis follows the more conventional phylogenetic placement of Proconsul within Hominoidea.

Early Miocene hominoids assumed to reflect the basal condition all display some type of postcranial apomorphies, but none appear to have the forelimb or thoracic specializations that characterize extant hominoids and Late Miocene hominoids (review in Ward 2007). The best-known early hominoid is Proconsul ( $P$. africanus, $P$. nyanzae, P. heseloni, P. major; 17-20 Ma) from multiple sites in Kenya and Napak in Uganda. Proconsul species have dental morphology distinct from one another but the postcrania are quite similar and are most easily distinguished on a basis of size, as well as location of discovery. Proconsul major (19-20 Ma; Koru, Songhor, Napak) was as large as a 
gorilla, estimated to be 63-87 kg (Rafferty et al. 1995) ${ }^{2}$. Proconsul africanus (18 Ma; Koru) had a body size between a gibbon and chimpanzee, between 12-27 kg (Gingerich 1977; Gingerich et al. 1982; Conroy 1987). Proconsul nyanzae (17-18.5 Ma; Rusinga Island, Mfangano Island) was approximately the size of a chimpanzee, 18-31 kg. (Conroy 1987) or maybe even as large as 40 kg (Walker et al. 1983; Walker and Pickford 1983). Proconsul heseloni (17-18.5 Ma; Mfangano Island, Rusinga Island, Kaswanga Primate Site) was similar in size to $P$. africanus, weighing 9-20 kg (Harrison 2002).

Most regions of the skeleton are preserved for Proconsul, with multiple partial skeletons known from P. nyanzae and P. heseloni (e.g., Figure 2.7). Proconsul nyanzae hip morphology is primarily known from multiple specimens from Kenya (KNM-RU 1753, KNM-RU 5527, KNM-RU 1633, KNM-MW 13142). All specimens are characterized by a moderate to high neck-shaft angle (mean $130^{\circ}$ per MacLatchy 2000), a robust femoral shaft, a prominent crista trochanterica on the posterior aspect of the femoral neck, and the trochanteric fossa is closed off posteriorly by a ridge of bone (Rose et al. 1992). The Proconsul nyanzae (KNM-MW 13142) male femur from Mfangano Island, Kenya ( 17.8 $\mathrm{Ma}$ ) is the most discussed femur because of its completeness. The KNM-MW 13142 femur has a spherical femoral head that is large relative to the femoral neck and a high neck-shaft angle (134') (Ruff et al. 1989; Ward 1991; Ward et al. 1993). The KNM-MW 13142 fovea capitis is described as intermediate between the proximally-positioned fovea in apes and the distally-positioned fovea in monkeys (Ward et al. 1993). There is some indication that the KNM-MW 13142 articular surface does not extend as equally in the posteroinferior direction as in Pan (MacLatchy and Bossert 1996).

\footnotetext{
${ }^{2}$ Some authors consider the fossils from Napak as a separate genus, Ugandapithecus (Senut et al. 2000; Gommery et al. 2002; Senut et al. 2004).
} 
Proconsul torso and forelimb morphology suggest a pronograde above-branch quadrupedal locomotor profile (Le Gros Clark and Leakey 1951; McHenry and Corruccini 1983; Walker and Pickford 1983; Beard et al. 1986; Ward 1993; Ward et al. 1993; Kelley 1997; Ward 2007). The back of Proconsul was long, with elongate vertebral bodies, more similar to cercopithecoid monkeys than extant apes (Ward 1991; 1993; Ward et al. 1993). Likewise, the ribcage was narrow and dorsoventrally deep and the pelvis was narrow with laterally-facing ilia (Ward 1991; 1993; Ward et al. 1993). However, Proconsul had long manual and pedal phalanges with strong flexor insertions, indicating a powerful grip (Begun 1993; Begun et al. 1994), high ranges of possible forearm pronation and supination (Kelley 1997; Rose 1997), and also lacked a tail (Ward et al. 1991; Nakatsukasa et al. 2004). It has been hypothesized that tail loss in hominoids is associated with powerful manual and pedal grasping associated with deliberate, cautious arboreal behaviors, reducing the importance of the tail for balancing during rapid and/or frequent leaping (Cartmill and Milton 1977; Begun et al. 1994; Kelley 1997; Rose 1997; Ward 2007).

Other fossil Miocene apes provide an opportunity to test the hypothesis that early apes have features consistent with below-branch species. Afropithecus (A. turkanensis; 17-17.5 Ma) is a hominoid with dental features distinct from Proconsul (Ward 1998; Deane 2012), although known postcranially from only a few elements from the sites of Kalodirr and Buluk (Leakey and Walker 1985; Leakey et al. 1988). The femora of Afropithecus turkanensis (17-17.5 Ma) are known from two femoral heads from Buluk (Leakey and Walker 1985; Leakey and Leakey 1986; Leakey et al. 1988). KNM-WS 12604 is an eroded femoral head and a small portion of femoral neck, whereas KNMWS 12605 is an eroded femoral head. These femoral heads have been largely ignored 
because of the weathering of the subchondral bone, which has made functional inferences nearly impossible. Overall, the Afropithecus postcrania suggest a chimpanzee-sized body ( $-35 \mathrm{~kg}$ ) (Harrison 2002), and are described as similar to $P$. nyanzae and P. africanus (Leakey and Walker 1985; Leakey and Leakey 1986; Leakey et al. 1988).

Morotopithecus (M. bishopi; 20.6 Ma) possesses primitive cranial features similar to the younger Afropithecus but displays derived features associated with orthograde locomotion, including a dorsally-positioned lumbar transverse process, oval scapular glenoid and mobile knee joint (Ward 1993; MacLatchy et al. 2000; MacLatchy 2004). Other features such as a tall greater trochanter, short femoral neck, and small femoral head suggest more limited range of motion at the hip joint (MacLatchy et al. 2000; MacLatchy 2004), which would be inconsistent with arboreal orthogrady. The femur of Morotopithecus bishopi is known from two femora from the same individual (Gebo et al. 1997; MacLatchy et al. 2000), with the right femur being relatively complete and preserving the greater and lesser trochanters, femoral neck, a separate but adjoining femoral head, three distal diaphyseal sections, and a condylar portion (MacLatchy et al. 2000). Since the original description in 2000, the adjoining pieces of the right proximal femoral shaft have been discovered (MacLatchy and Kingston 2013). The proximal femoral morphology has been interpreted as favoring fore and aft movements and little abduction (MacLatchy et al. 2000). The MUZ-M80 right femoral neck shaft angle is high (traditional NSA $135^{\circ}$ ) (MacLatchy et al. 2000) but this is seemingly countered by a small femoral head relative to neck dimensions, a short femoral neck, and a greater trochanter that approximates the superior aspect of the femoral head. In addition, the fovea capitis is not centrally located and is instead more 
posteroinferiorly positioned on the subchondral bone as in monkeys (MacLatchy and Bossert 1996; MacLatchy et al. 2000). The Morotopithecus right femur is unusual among African Miocene hominoids in that the femoral head is not anteriorly displaced or anteverted (MacLatchy et al. 2000; Nakatsukasa et al. 2012).

If the Morotopithecus postcranials are indeed 20.6 Ma and derived for orthograde behaviors, this presents several intriguing scenarios. The first scenario is that Morotopithecus is a crown hominid (i.e., great apes, see Figure 1.1), which would mean that the divergence date of hylobatids has been underestimated and that hominids evolved earlier than originally thought (Young and MacLatchy 2004). The second possibility is that similarities between extant nonhuman hominids and Morotopithecus are the result of homoplasy. Neither scenario can currently be falsified with the fossil data available (MacLatchy et al. 2000; MacLatchy 2004; Young and MacLatchy 2004).

Nacholapithecus kerioi ( $15 \mathrm{Ma})$ is a particularly enigmatic ape, differing from all other hominoids in having a narrow trunk coupled with relatively small hindlimbs and strikingly large forelimbs (Ishida et al. 2004; Nakatsukasa et al. 2007; Nakatsukasa and Kunimatsu 2009) (Figure 2.8). Prior to the discovery of Nacholapithecus, all known hominoids with enlarged forelimbs also had the characteristic torso rearrangement associated with orthogrady. However, the small monkey-like vertebral bodies, humeral trochlea, and unspecialized phalanges of Nacholapithecus seem to indicate pronogrady (Ishida et al. 2004; Nakatsukasa et al. 2007; Nakatsukasa and Kunimatsu 2009).

Nacholapithecus kerioi hip morphology is known from a few isolated femoral head fragments and a partial skeleton (KNM-BG 35250), which preserves portions of the femora and a partial ischium (Ishida et al. 1999; Ishida et al. 2004). The KNM-BG 35250 right femur is the most complete of the Nacholapithecus femora, although suffering from 
some plastic deformation distally. This femur has a short femoral neck and high neckshaft angle $\left(140^{\circ}\right)$, with a femoral head that extends just proximally to the greater trochanter (Ishida et al. 2004; Nakatsukasa et al. 2012). The femoral head is strongly anteverted, to such an extent that it is attributed to deformation (Nakatsukasa et al. 2012). The articular surface contours are well preserved, missing only a small portion posteriorly, and the articular surface appears to "mushroom" over the neck (Nakatsukasa et al. 2012). The fovea capitis is positioned posteroinferiorly to the equator of the femoral head (Nakatsukasa et al. 2012), in a similar position as Proconsul nyanzae (KNM-MW 13142). The hip joint thus has a combination of morphologies which are hypothesized to both enhance (i.e., high neck-shaft angle, femoral head projects above trochanter, expansive articular surface) and limit (i.e., short femoral neck, non-centrally positioned fovea) hip joint mobility.

Equatorius africanus ${ }^{3}$ is a large hominoid species with postcrania known from the Maboko Formation, the Nachola Formation, and the Tugen Hills of Kenya (14-15.5 Ma) (Ward et al. 1999; Ward and Duren 2002). This species appears to have been similar to early arboreal hominoids, having a long flexible back, ulnar styloid contact with the wrist, and a retroflexed humerus (Ward et al. 1999). Equatorius was probably semi-terrestrial based on limited shoulder mobility, a retroflexed olecranon, terrestrial hand proportions, and relatively straight phalanges that are robust (Ruff et al. 1989; Benefit and McCrossin 1995; McCrossin and Benefit 1997; Sherwood et al. 2002; Patel et al. 2009). A femur attributed to Equatorius africanus (BMNH 16331, previously attributed to Proconsul and Kenyapithecus) is known from Maboko Island, Kenya (14-15.5 Ma) (Le Gros Clark and Leakey 1951; Rose et al. 1996). The femur preserves the femoral head and neck,

\footnotetext{
3 Junior synonyms include Kenyapithecus africanus Leakey 1967and Griphopithecus africanus Begun 1987.
} 
lesser trochanter and much of the anterior aspect of the greater trochanter, and the most proximal portion of the diaphysis. The neck-shaft angle is moderate (125) (MacLatchy et al. 2000) to high $\left(130^{\circ}\right)$ (Bacon 2001). The Equatorius femur is described as having a long femoral neck, high greater trochanter, and a femoral head to neck ratio that is smaller than Proconsul (Benefit and McCrossin 1995). The articular surface is highly spherical and extends anteriorly and posteriorly to smoothly transition into the femoral neck (Rose 1983), although there is some damage to the anterosuperior aspect of the articular surface. The articular profile is similar to Sivapithecus and Proconsul (MacLatchy and Bossert 1996), and the fovea capitis is relatively centrally-positioned. The Maboko Island femur has been likened to ambling, palmigrade arboreal quadrupeds (McCrossin and Benefit 1994), which would suggest a locomotor repertoire with no extant homolog if paired with terrestrial knuckle-walking.

Kenyapithecus wickeri is also a large hominoid species, known from specimens recovered at Fort Ternan, Kenya (14 Ma) (Simons 1969; Andrews and Walker 1976). Extensive dental and some mandibular materials are known for Kenyapithecus, but the morphology is more derived than specimens attributed to Equatorius (Pickford 1985; Harrison 1992; Ward et al. 1999; Sherwood et al. 2002). The only postcranial element referred to Kenyapithecus is a distal unassociated humerus, characterized by a wide trochlea relative to capitulum breadth, pronounced lateral trochlear keel, prominent supracondylar crest, deep olecranon fossa, small and posteriorly oriented medial epicondyle, and a shaft that is oval is cross-section (Simons 1969; Andrews and Walker 1976; Morbeck 1983; Ward and Duren 2002). Little can be inferred from this humerus other than that it was probably capable of powerful flexion-extension movements. 
Several middle Miocene taxa are found outside Africa, the earliest being Griphopithecus. Griphopithecus, known primarily from chimpanzee-sized thicklyenameled molars discovered in Slovakia, Austria, Germany, and Turkey (Abel 1902; Heizmann and Begun 2001; Holec and Emry 2003), is hypothesized to have been closely related to Equatorius based on the dental morphology (Begun 2000; 2002) ${ }^{4}$. The Griphopithecus material is potentially as old 16.5 Ma (Heizmann and Begun 2001; Begun et al. 2003a; Begun et al. 2003b), although there is an argument for a later appearance for European hominoids at $14 \mathrm{Ma}$ (Böhme et al. 2011; Casanovas-Vilar et al. 2011), which seems in better accord with timing of Equatorius-Kenyapithecus in Africa (Feibel and Brown 1991; Pickford et al. 2006). Although little is known of Griphopithecus, the association with Kenyapithecus could indicate that this taxon was either not a great ape at all (Begun 2002) or might be a plausible hominid ancestor (Begun 2007). Postcrania have been recovered at Paşalar in Turkey, although the only material published are phalanges consistent with mixed semi-terrestrial and semiarboreal locomotor behaviors (Ersoy et al. 2008). A partial ulna and robust humerus from Klein Hadersdorf, Austria (Ehrensberg 1938) have been attributed to Griphopithecus based on similarities with Kenyapithecus-Equatorius (Begun 2002), and these forelimb elements are compatible with generalized quadrupedalism (Begun 2002; Alba et al. 2010b). However, it will remain unclear what locomotor behaviors characterized the earliest expansion of apes into Eurasia until associated dental and postcranial materials are recovered.

\footnotetext{
${ }^{4}$ Begun (2000) hypothesizes that there is also a Eurasian connection to Kenyapithecus in addition to Equatorius. Griphopithecus alpani (Devínska Nová Ves in Slovakia, Paşalar and Çandır in Turkey) is linked to Equatorius africanus (Kipsaramon, Maboko, Nachola, and Tugen Hills, Kenya), with Kenyapithecus wickeri (Fort Ternan, Kenya) linked to a putative second hominoid species (c.f. Kenyapithecus kizili) at Paşalar, Turkey.
} 
Sivapithecus (S. parvada, S. indicus, S. sivalensis; 9-12 Ma) from the Siwaliks formation in India and Pakistan is a putative fossil pongine based on facial morphology (Ward and Kimbel 1983; Ward and Pilbeam 1983). Sivapithecus parvada is thought to have a monkey-like torso and humerus and has been interpreted on this basis to not have had forelimb-dominant locomotion like Pongo, possibly implying independent evolution of great ape-like below branch arboreality in pongines and hominines (the "Sivapithecus dilemma"-- Pilbeam et al. 1990; Ward 1997; Larson 1998; Pilbeam and Young 2001; Madar et al. 2002; Young 2003).

Sivapithecus sivalensis proximal femur and pelvic morphology is known from isolated remains found in the Siwalik exposures. Little can be said about the Sivapithecus pelvis until the specimen is fully described, but early reports suggest it is "least unlike Proconsul nyanzae"(Morgan et al. 2011). Specimen YPM-GSP 12654 is a femoral neck and intertrochanteric region of a left femur. It has a steeply inclined femoral neck that is extremely anteroposteriorly narrow, a characteristic of all Sivapithecus specimens preserving a femoral neck (Pilbeam et al. 1980). Three femoral heads (YPM-GSP 13929, YPM-GSP 11867, YPM-GSP 15782) are attributed to S. sivalensis, although little can be said about these. Specimen YPM-GSP 11867 is a left proximal femoral head with a portion of the femoral neck, preserving a minimally abraded articular surface with distinct articular borders. The YPM-GSP 11867 fovea capitis is extremely shallow but expansive, and somewhat posteroinferiorly displaced on the subchondral surface, and the femoral neck is very anteroposteriorly narrow. Specimen YPM-GSP 13929 and YPM-GSP 15782 preserve foveal morphology but it is difficult to estimate whether the fovea was centrally-positioned. The YPM-GSP 15782 fovea capitis is deeper than YPM-GSP 11867 and similar to that observed in all non- 
pongine anthropoid primates. Overall, the Sivapithecus femoral morphology has been taken to indicate a high amount of hip abduction and lateral rotation abilities(Pilbeam et al. 1980; MacLatchy and Bossert 1996), which is somewhat inconsistent with pronogrady. The disjunct between pongine forelimb and hindlimb morphology might indicate that the hindlimb was the initial target of selection in pongine orthograde behaviors, contrary to Morotopithecus and Nacholapithecus.

Pierolapithecus catalaunicus (11.9 Ma; Spain) is a stem great ape with adaptations in the vertebrae and ribcage that suggest more frequent use of orthograde behaviors (Moyà-Solà et al. 2004; Susanna et al. 2010) (Figure 2.9). It has been inferred that Pierolapithecus was not suspensory based on phalanges that are relatively short and not strongly curved (Almécija et al. 2009; Alba et al. 2010a; but see Deane and Begun 2008, 2010). A recent study of the Pierolapithecus pelvis fragments suggests that this species maintained a pelvis structure generally similar to the early hominoid Proconsul nyanzae (Hammond et al. 2013a), and not unlike the morphology described for Sivapithecus (Morgan et al. 2011).

Dryopithecus fontani $^{5}$ (11-12 Ma) is primarily known from three partial mandibles and two isolated teeth from France (Lartet 1856) and a maxilla from Spain (Moyà-Solà et al. 2009), although other isolated skeletal and dental elements are attributed to this taxon. The Dryopithecus hypodigm once included most hominoid material in Europe but has recently undergone substantial revision, with most postcranial remains re-assigned

\footnotetext{
${ }^{5}$ The type-specimen of a second species, Dryopithecus brancoi, was named after a single molar from Salmendigen, Germany (Schlosser 1901; Abel 1931; Szalay and Delson 1979). Molars from Rudabánya, Hungary share characters with the Salmendigen molar and thus the Rudabánya hominoid species was referred to as Dryopithecus brancoi (Begun and Kordos 1993). However, the discovery of several new European middle and late Miocene crania led to generic revisions (Begun et al. 2008). The genus Rudapithecus (Kretzoi 1969) has been resurrected for the hominoid material from Rudabánya, Hungary (Begun 2006; Begun et al. 2008), with Hispanopithecus (Villalta Comella and Crusafont Pairó 1944) formally resurrected for Spanish materials (Moyà-Solà et al. 2009). In addition, the taxonomic status of the Dryopithecus brancoi type specimen is unresolved (Begun et al. 2006), with some concern that the Salmendigen molar may not belong to a hominoid (Andrews et al. 1996).
} 
to Hispanopithecus-Rudapithecus (Begun 2006; Almécija et al. 2007; Begun et al. 2008; Ward et al. 2008; Moyà-Solà et al. 2009; Casanovas-Vilar et al. 2011; Begun et al. 2012). Pollical phalanges probably belonging to $D$. fontani discovered in Castell de Barberà (Spain) are broadly similar to all other Miocene apes and suggest powerful grasping abilities (Almécija et al. 2011). A partial femur (IPS-41724) from Abocador de Can Mata (Spain) tentatively attributed to Dryopithecus fontani has features typically associated with pronograde quadrupedalism, including a posteriorly directed femoral head, tall greater trochanter, and a low femoral neck shaft angle (Moyà-Solà et al. 2009; Alba et al. 2010b; Pina 2011). The IPS-41724 femur is attributed to Dryopithecus because it is unlike the femora of Hispanopithecus (see below) and generated body size estimates (44kg) that are larger than the body mass estimated for the other Miocene ape from this region, Pierolapithecus catalaunicus. The IPS-41724 femur has a neck-shaft angle estimated to be somewhat low $\left(123^{\circ}\right)$ (Moyà-Solà et al. 2009), with a spherical femoral head is large relative to the femoral neck (Pina 2011) and positioned low relative to the greater trochanter (Moyà-Solà et al. 2009). Taken together, these features suggest more limited hip mobility than some of the other Miocene taxa from Europe. Likewise, a humerus from the Saint Gaudens type-site in France (Lartet 1856; Le Gros Clark and Leakey 1951; Pilbeam and Simons 1971) and a humerus from Castell de Barberà in Spain (Alba et al. 2010b; Alba 2012) have both been tentatively attributed to D. fontani. These humeri are consistent with quadrupedal behaviors, and seem to differ from the Griphopithecus humerus in having a less triangular cross section, a more developed lateral supracondylar ridge, and a medial and anterior concavity to the shaft (Alba et al. 2010b). Although the postcranial evidence is limited, Dryopithecus may have been less orthograde than the late European fossil apes. 
Hispanopithecus (H. laietanus, $H$. crusafonti) (9.5 Ma; Spain) is a late Miocene European ape but is argued to have highly derived forelimbs and hands, suggesting a strong suspensory component to its locomotor repertoire (Moyà-Solà and Köhler 1996; Almécija et al. 2007; Almécija et al. 2009; Alba et al. 2012). The hands are long (Figure 2.9), with long curved phalanges with strong flexor insertions and robust metacarpals built for powerful grasping (Moyà-Solà and Köhler 1996; Almécija et al. 2007; Almécija et al. 2009). The lumbar vertebrae have transverse processes originating on the pedicle and the spinous process is caudally oriented, indicating reduced mobility (Moyà-Solà and Köhler 1996). The forelimb bones are elongate and provide extensive areas for forelimb muscular attachment, with the ulna having reduced contact with the wrist and allowing for more mobility at this joint (Moyà-Solà and Köhler 1996; Alba et al. 2012). Although pelvic material is unknown for Hispanopithecus, two femora, both nearly $2 / 3$ complete, are preserved (Moyà-Solà and Köhler 1996). The femoral head extends above the greater trochanter, the neck-shaft angle is high $\left(132^{\circ}\right)$, and the breadth of the femoral head is large relative to the neck (Moyà-Solà and Köhler 1996; Köhler et al. 2002; Moyà-Solà et al. 2009). The femora are argued to have a homogeneous cortical distribution in the neck that is most similar to suspensory apes (Pina et al. 2012), with a high neck-shaft angle $\left(132^{\circ}\right)$ and a proportionately large femoral head that should promote hip mobility (Moyà-Solà and Köhler 1996).

Rudapithecus hungaricus (formerly D. brancoi) from Rudabánya, Hungary (10 Ma) preserves postcranial elements from the elbow, hip, hand, knee, ankle, and foot (Kretzoi 1975; Morbeck 1983; Begun 1993; Begun et al. 2012). Like other middle and late Miocene taxa, this species was highly dimorphic postcranially, with males approximately twice as large as females (Begun and Kordos 2011). Rudapithecus 
hungaricus displays similar features as the contemporaneous Hispanopithecus but differs in having a less concave frontal bone (Begun 1994), less developed temporal lines, and less robust manual proximal phalanges (Begun et al. 2012). Because Hispanopithecus and Rudapithecus only differ by a few characters, they have been suggested to be sister taxa with similar locomotor adaptations (Begun et al. 2012) or the same genus (Casanovas-Vilar et al. 2011; Alba 2012), but the debate will not be resolved until additional material common to both taxa are recovered.

Rudapithecus hungaricus appears to have been suspensory. Rudapithecus hungaricus has long and curved phalanges (Figure 2.10), features attributed to high elbow mobility, proximal femoral morphology described as similar to Hispanopithecus, and scaphoid and capitate morphology most similar to Pongo and Hispanopithecus (Begun 1992; 1993; Kivell and Begun 2009; Begun and Kordos 2011; Begun et al. 2012). The forelimb bones are large relative to femoral head diameter (Morbeck 1983). The pelvis and femur of Rudapithecus have not been formally described (but see Begun and Kordos 2011; Begun et al. 2012) but initial work suggests that the pelvis displays transverse flaring as in extant suspensory apes (Ward et al. 2008). Several hominoid femora are known from Rudabánya, and these femora are characterized by "a large head, low neck-shaft angle, and a robust, curved and platymeric shaft" (Begun and Kordos 2011). A right (RUD 184) and a left femur (RUD183) (Kordos and Begun 2001) of similar size are likely from the same individual, and are associated with an undescribed pelvis (see Chapter 5). The right femur (RUD 184) is mostly complete proximally but missing the distal end and the greater trochanter, and suffering from some crushing in the diaphysis (Begun et al. 2012). The left femur (RUD 183) is a partial femoral head and proximal portion of the femoral neck. A large femur, probably from a 
male, is roughly twice as large as the other femora (Begun and Kordos 2011). Additional morphological assessments cannot be made until these femora are formally described, but the morphologies attributed to these specimens are consistent with morphologies attributed to high joint mobility.

Oreopithecus bambolii (6.7-8.3 Ma) was the latest known ape in Europe, surviving past the Vallesian extinction event ( 9.6 Ma) responsible for eliminating the other hominoids in Europe (Rook et al. 2011). The majority of specimens come from Baccinello and Monte Bamboli in Tuscany (Gervais 1872; Hürzeler 1949; 1958), Sardinia (Cordy and Ginesu 1994), and potentially from Moldova as well (see discussion in Delson 1986 and references therein). It is one of the most well-represented fossil apes, with materials from multiple individuals and most regions of the body. A remarkably complete, although distorted, skeleton (IGF 11778) has allowed estimations of body mass (32 kg.) and intermembral index (Jungers 1987; 1990).

Historically, locomotor behaviors used by Oreopithecus have been highly disputed although there is a growing consensus that Oreopithecus was an orthograde suspensory ape (Schultz 1960; Straus 1963; Jungers 1987; Sarmiento 1987; Harrison 1991; Harrison and Rook 1997; Rose 1997; Begun 2002; Susman 2004; Ward 2007; Russo and Shapiro 2013) or perhaps had evolved from a suspensory species (Köhler and Moyà-Solà 1997; Rook et al. 1999). Support for Oreopithecus using forelimb dominant locomotion comes from its high intermembral indices matched only by orangutans (Jungers 1987) with large articular dimensions of the limb joints (Schultz 1960; Harrison 1986). However, Oreopithecus has shorter hands (Jungers, 1987; MoyàSolà et al., 1999; Rook et al., 1999; but see Susman, 2004) and less specialized wrist bones (Harrison 1986) than living suspensory apes and Hispanopithecus (Almécija et al. 
2007). The Oreopithecus femur has been suggested to have a human-like bicondylar angle (Kummer 1965; Köhler and Moyà-Solà 1997), although the specific femur in question (IGF 11778) suffers from severe diagenetic compression throughout except for the well-preserved femoral head. Because both bipedal and suspensory species use hindlimb extension and display a large femoral head on a steeply inclined femoral neck (e.g., Harmon 2009), and because Oreopithecus lacks adaptations for suspension in the phalanges, there are still some lingering questions about bipedalism in Oreopithecus (Harrison 1991; Köhler and Moyà-Solà 1997; Rook et al. 1999).

Several specimens from the hip are preserved for Oreopithecus bambolii, with multiple partial femora and a deformed pelvis known(Harrison 1986; 1991). Unfortunately, these specimens are typically too fragmentary or distorted to be highly informative regarding hip function. The most well-known Oreopithecus specimen is IGF 11778 , which displays features thought to facilitate a wide range of rotation and full extension at the hip joint, indicated by a large, globular femoral head positioned on a long and steeply-inclined neck, a centrally-positioned fovea capitis, and a well-developed iliofemoral ligament insertion site on the pelvis (Harrison 1991). Another Oreopithecus femur, IGF $2011 \mathrm{~V}$, is a left proximal femur preserving a greater trochanter, femoral neck, and femoral head. The IGF $2011 \mathrm{~V}$ articular surface is beautifully preserved and complete, with a deep fovea capitis centrally located on the subchondral bone. Additionally, there are Oreopithecus specimens (BA74, BA75, BA86, BA128) from the Baccinello lignite mines of Tuscany housed in Basel that are in the process of being described. These specimens are just femoral heads, all of which are characterized by an extremely deep fovea capitis that is centrally located on the subchondral surface. All 
of the features described for Oreopithecus femora are compatible with high levels of mobility at the hip joint.

\section{Problems in understanding evolution of fossil apes}

One of the biggest obstacles to reconstructing locomotor abilities in Miocene apes is that there is no extant analog for them, and it is difficult to characterize their

anatomy as either primitive or derived. All later Miocene forms (e.g., Hispanopithecus, Rudapithecus, Oreopithecus) appear to have suspensory adaptations, although not as highly derived as the extant apes. This has led some to speculate that the morphological and behavioral adaptations of the extant hominoids, particularly the hominids, are specialized patterns that have evolved somewhat recently (Harrison 1991; Larson 1998; Moyà-Solà et al. 2004; Lovejoy 2009; Lovejoy et al. 2009a; Lovejoy et al. 2009b). As Miocene hominoids are a surprising mixture of monkey-like and extant apelike features (e.g., Harrison 1991; MacLatchy and Bossert 1996; Moyà-Solà and Köhler 1996; McCrossin and Benefit 1997; Ward et al. 1999; MacLatchy et al. 2000; Ishida et al. 2004; Moyà-Solà et al. 2009), functional analyses that have attempted to elucidate locomotor abilities based on comparisons to highly derived extant ape and cercopithecid morphologies are almost certainly lacking the resolution necessary to identify what kinds of behaviors these animals were actually capable of using. Proconsul nyanzae, for instance, has features assumed to both limit (e.g., small femoral head, tall greater trochanter, non-centrally positioned fovea capitis) and reflect high hip abduction abilities (e.g., a large acetabular fossa, large neck shaft angle) (Ward et al. 1993), and has been interpreted as an ambling, clambering above-branch quadruped (Kelley 1995). It is unclear how different morphological combinations work together to produce, or reflect, functional abilities. 
Moreover, the work by Chan (Chan 2007b; a; 2008) has posed difficult questions for the trait-function association in extant species (for broader scope, see also Hickman 1988; Van Valkenburgh 1994; Lauder 1995; Koehl 1996). When testing the assumption that suspensory primates have an increased range of motion at the shoulder joint to facilitate below-branch behaviors, Chan found that the hominoid glenohumeral joint does not actually produce a greater range of circumduction than other primates (Chan 2007a), nor does the shape of the shoulder joint and thorax necessarily promote increased mobility (Chan 2007b). Additionally, Chan found that the same level of mobility was achieved via different combinations of morphologies in different species (e.g., hominoids vs. lorines) and that different morphologies can even "cancel each other out" (Chan 2008). Chan's findings reaffirm that joint function, and the relationship between mobility and anatomical form, must be approached as a system.

\section{MODELING JOINT FUNCTION}

The only method for evaluating hip joint function as a system in fossil hominoids is by modeling hip joint movement using both the acetabulum and femur. The theory for modeling hip joint abduction was outlined by MacLatchy (MacLatchy 1995; 1996; MacLatchy and Bossert 1996; MacLatchy 1998). Although MacLatchy's digital sample was small by today's standards, her 3D techniques were extremely sophisticated and show much foresight. The methodologies for hip joint modeling described later in this thesis derive primarily from MacLatchy's (1995) thesis work, from which her subsequent publications are based (1996, 1998; and Bossert, 1996).

MacLatchy summarized the limits of how the hip joint should be positioned during loading based on joint functional biology (Kapandji 1970; Jenkins 1972; Jenkins and Camazine 1977; Latimer et al. 1987; Ward 1991; 1993; Ward et al. 1993) and used this 
framework to conduct a study of hip joint abduction in primates. MacLatchy's digital modeling technique aligned the femoral head within the acetabulum using centroids of best-fit spheres fit to the femoral head and lunate surface within a sample of anthropoids (Pan, Pongo, Homo, Mandrillus, Macaca) and prosimians (Galago, Perodicticus, Lepilemur, Cheirogaleus, Microcebus). She maximally abducted the femur in positions of flexion and extension following 3 boundary constraints (1995, p. 116). First, during simulations, the fovea capitis was limited to the confines of the acetabular fossa. Second, the greater trochanter was not allowed to intersect the pelvis. Third, MacLatchy allowed the subchondral borders of the femoral head and lunate surface to only "slightly" overlap, using Jenkins and Camazine (1977) as a guide for normal joint boundary positions.

MacLatchy's study found a spectrum of abduction capabilities in anthropoids corresponding to what is known about their locomotor behaviors. As would be predicted, abduction was consistently highest in Pongo, followed by Pan. In the flexed position, humans have the most limited range of abduction, followed by the monkeys and chimpanzees. During extension, range of abduction separates humans and monkeys from the chimpanzees and orangutans. These differences were related to different hindlimb postures required during suspensory behaviors versus more generalized arboreality. When abduction was measured in Miocene hominoid Proconsul nyanzae, Proconsul appears similar to the monkeys during flexion but closer to chimpanzees during the extended posture, which suggests that Proconsul was probably capable of climbing postures that would be difficult for most cercopithecids. These results provided more specificity about hip use and locomotor abilities in Proconsul than was previously possible. 


\section{Model validation}

Models, such as those derived from imaging techniques (e.g., MRI, CT, optical and laser scanning), are anatomically realistic and complex 3D representations of a real structure. However, even biologically complex, high-fidelity models are simplifications of real-world systems. The utility of modeling as a tool thus depends on how well the model replicates reality. The only way to know how closely a model matches reality is by comparing it to empirical data, a procedure often called model validation (see reviews in Richmond et al. 2005; Hutchinson 2011). When modeling joint positions using just the bones, abstraction of the system is introduced by not including the overlying soft tissues (e.g., articular cartilage, ligaments, labrum, muscles). It is therefore imperative to assess the limiting influence that the absent soft tissues might have on the results (Hutchinson and Garcia 2002; Gatesy et al. 2009; Hutchinson 2011; Hutson and Hutson 2012; Pierce et al. 2012). This is particularly true for the hip joint, where mobility is determined primarily by soft tissues (Kapandji 1970; Wright and Radin 1993; Levangie and Norkin 2005; Safran et al. 2012).

The regulations associated with living nonhuman primates makes in vivo validation of primate models difficult. Not surprisingly, MacLatchy was limited in her ability to collect live animal measures to test the accuracy of her models. MacLatchy $(1995 ; 1998)$ gathered in vivo abduction data for 4 prosimian species ( $n=8 ; 2$ individuals per species) that she modeled abduction in, and was unable to collect in vivo data on anthropoids. MacLatchy's in vivo data points are within a single standard deviation of the mean abduction during flexion, but abduction during extension does not as closely correspond to the mean values. Although a relationship can be tentatively inferred from these 
limited data points, the small in vivo sample size and taxonomic composition prevent confirmation that MacLatchy's models are representative of her in vivo data. 
Table 2.1 Comparison of percentages of adult locomotion in extant anthropoids

\begin{tabular}{|c|c|c|c|c|c|c|}
\hline Species & $\begin{array}{c}\text { Arboreal } \\
\text { quadrupedal }\end{array}$ & $\begin{array}{c}\text { Terrestrial } \\
\text { quadrupedal }\end{array}$ & Climbing & $\begin{array}{c}\text { Suspension } \\
\text { (includes } \\
\text { brachiation) }\end{array}$ & Leap & Other \\
\hline Hylobates $^{1}$ & & & $21 \%$ & $56 \%$ & $15 \%$ & $8 \%$ bipedalism \\
\hline $\begin{array}{l}\text { Symphalangus } \\
\text { syndactylus }^{2}\end{array}$ & & & $37 \%$ & $51 \%$ & $6 \%$ & $6 \%$ bipedalism \\
\hline Pongo abelii ${ }^{3}$ & $18 \%$ & & $26 \%$ & $16 \%$ & & $\begin{array}{c}22 \% \text { clambering, } \\
7 \% \text { tree sway }\end{array}$ \\
\hline Pongo pygmaeus ${ }^{4}$ & $12 \%$ & & $18 \%$ & $12 \%$ & & $\begin{array}{c}51 \% \text { clambering, } \\
7 \% \text { tree sway }\end{array}$ \\
\hline Gorilla beringei $^{5}$ & & $97 \%$ & $<2 \%$ & $<1 \%$ & & \\
\hline Gorilla gorilla $^{6}$ & \multicolumn{2}{|c|}{$64 \%$} & $20 \%$ & $5 \%$ & & $\begin{array}{c}3 \% \text { transfer, } 6 \% \\
\text { bipedalism }\end{array}$ \\
\hline Pan troglodytes ${ }^{7}$ & \multicolumn{2}{|c|}{$22 \%$} & $68 \%$ & $7 \%$ & & $3 \%$ bipedalism \\
\hline Pan paniscus ${ }^{8}$ & \multicolumn{2}{|c|}{$35 \%$} & $50 \%$ & $9 \%$ & $3 \%$ & \\
\hline Pygathrix nemaeus ${ }^{9}$ & $54 \%$ & & & $46 \%$ & & \\
\hline Nasalis larvatus & * & & * & & * & \\
\hline $\begin{array}{l}\text { Rhinopithecus } \\
\text { roxellana }\end{array}$ & * & & * & & * & \\
\hline Trachypithecus sp. ${ }^{10}$ & $\sim 50 \%$ & & $\sim 10 \%$ & & $\sim 40 \%$ & \\
\hline Colobus guereza $^{11}$ & $39 \%$ & & $11 \%$ & & $44 \%$ & \\
\hline Piliocolobus badius & * & & & & * & \\
\hline Macaca fascicularis ${ }^{12}$ & $59 \%$ & $<2 \%$ & $12 \%$ & & $6 \%$ & $15 \%$ scrambling \\
\hline Macaca nemestrina & * & * & & & & \\
\hline Theropithecus gelada & & * & & & & \\
\hline Papio sp. ${ }^{13}$ & & $97 \%$ & $<1 \%$ & & & \\
\hline Cercopithecus mitis ${ }^{11}$ & \multicolumn{2}{|c|}{$54 \%$} & $37 \%$ & & $11 \%$ & \\
\hline Erythrocebus patas & & * & & & & \\
\hline Ateles paniscus ${ }^{14}$ & $25 \%$ & & $39 \%$ & $31 \%$ & $4 \%$ & \\
\hline Alouatta caraya ${ }^{15}$ & $69 \%$ & & $19 \%$ & & $3 \%$ & $10 \%$ bridging \\
\hline Cebus capucinus ${ }^{16}$ & $54 \%$ & & $26 \%$ & & $15 \%$ & \\
\hline
\end{tabular}

* indicates a known significant component of locomotor repertoire but unknown percentage values.

${ }^{1}$ Hunt (2004) calculated from Gittins (1982), Fleagle (1980), and Srikosamatara (1984), ${ }^{2}$ Hunt (2004) calculated from Fleagle (1980), ${ }^{3}$ Thorpe and Crompton (2006), ${ }^{4}$ Cant (1987) using data only for females, ${ }^{5}$ Doran (1996), ${ }^{6}$ Hunt (2004) calculated from Remis (1995), Doran (1996), ${ }^{8}$ Hunt (2004) calculated from arboreal locomotor bouts from Susman et al. (1980), Doran (1996), and Susman (1984), ${ }^{9}$ Byron and Covert (2004), ${ }^{10}$ Fleagle (1998) for Trachypithecus obscura, ${ }^{11}$ Gebo and Chapman (1995), ${ }^{12}$ Cannon and Leighton (1994), ${ }^{13}$ Hunt (1991), ${ }^{14}$ Mittermeier (1978) and Fleagle and Mittermeier (1980), ${ }^{15}$ Prates and Bicca-Marques (2008), ${ }^{16}$ Gebo (1992) . 
Table 2.2 Broad locomotor categorization of extant anthropoids considered in this study

\begin{tabular}{|l|l|l|}
\hline Category & $\begin{array}{l}\text { Reliance on suspensory } \\
\text { behaviors }\end{array}$ & Taxa \\
\hline Suspensory & $\begin{array}{l}\text { suspensory behaviors } \\
\text { dominant } \\
\text { suspensory behaviors } \\
\text { very important }\end{array}$ & Pan, G. gorilla, Ateles \\
\hline Unclassified & indeterminate & G. beringei, Alouatta, Pygathrix \\
\hline suspensory & $\begin{array}{l}\text { suspensory behaviors } \\
\text { rarely observed }\end{array}$ & $\begin{array}{l}\text { Rhinopithecus, Colobus, } \\
\text { Piliocolobus, Nasalis, } \\
\text { Trachypithecus, Cercopithecus, } \\
\text { Macaca, Cebus }\end{array}$ \\
& $\begin{array}{l}\text { suspensory behavior } \\
\text { extremely rare }\end{array}$ & $\begin{array}{l}\text { Papio, Erythrocebus, } \\
\text { Theropithecus }\end{array}$ \\
\hline
\end{tabular}




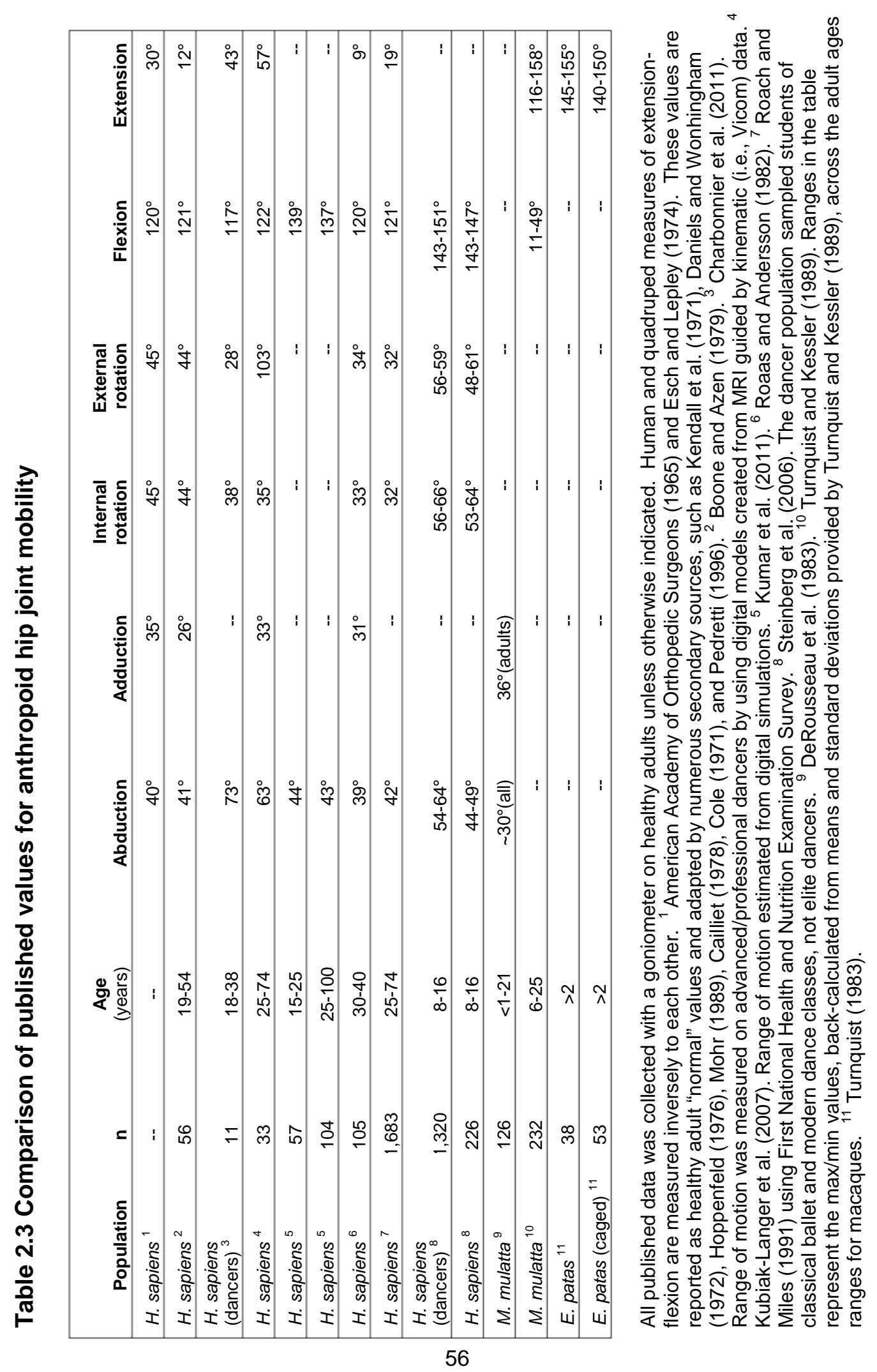




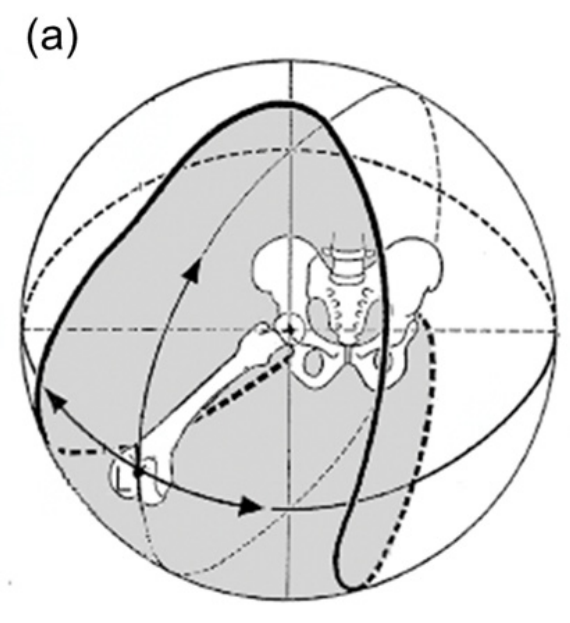

(b)

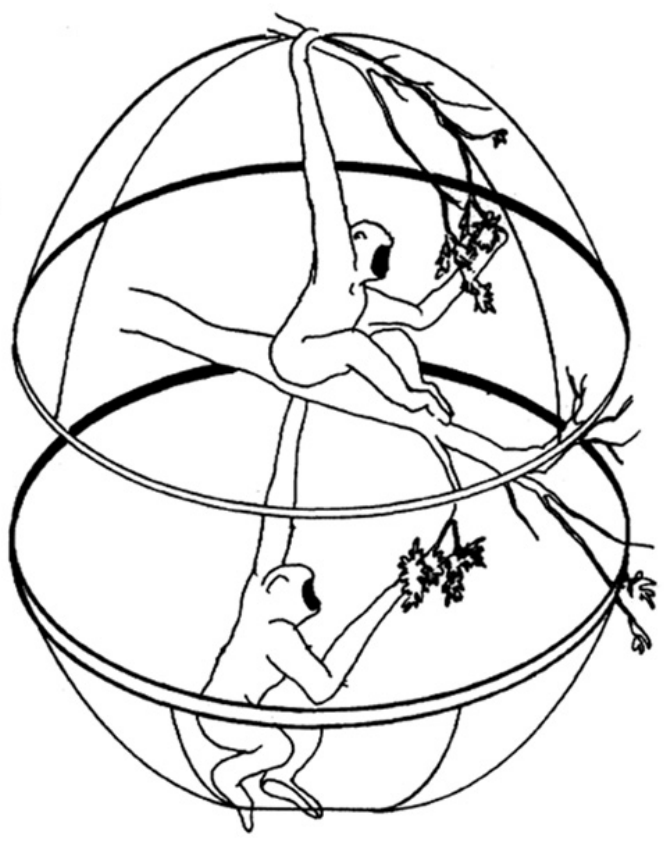

Figure 2.1 Large hindlimb spatial envelope increases the range of foot positions (a) The range of positions of the knee is a proxy for the hindlimb spatial envelope, or the overall range of foot positions. An increase in joint mobility and/or body size will increase the hindlimb spatial envelope. Image modified from Kapandji (1970). (b) An increase in the spatial envelope of the limbs will increase the range of supports, as well as the feeding space, available to an animal. Image modified from Grand (1972). 


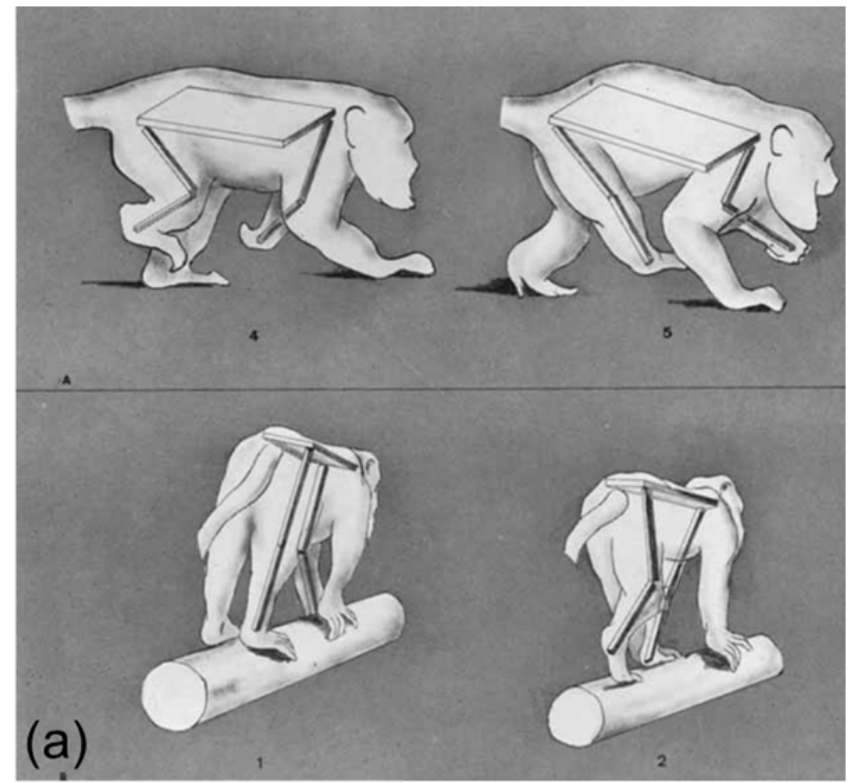

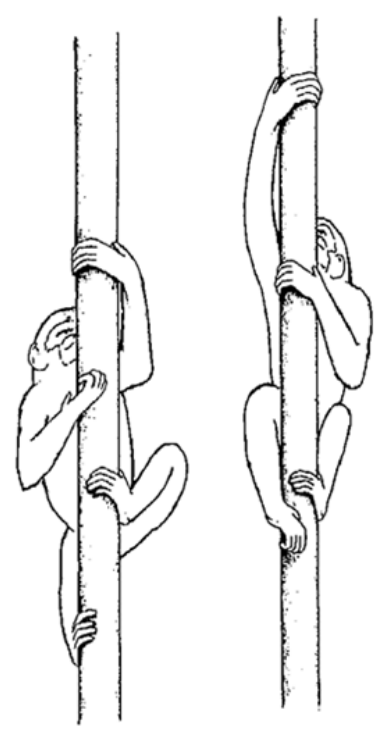

(b)

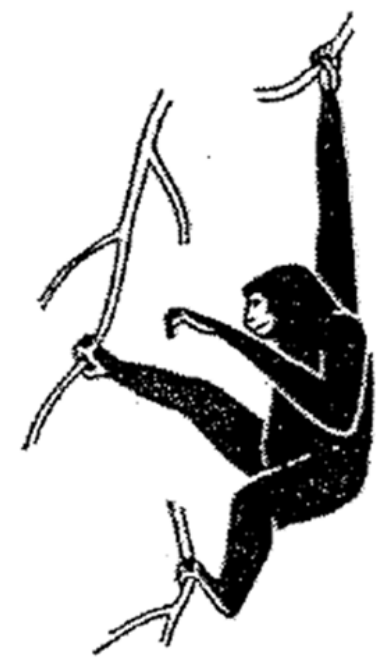

(c)

Figure 2.2 Hindlimb postures used by anthropoids during different locomotor behaviors

(a) Quadrupedal arboreal behaviors in Alouatta emphasize adducted and flexed thigh postures. Image modified from Grand (1968). (b) Vertical climbing in Pan elicits abducted and laterally rotated positions of the thigh. Image modified from Stern and Susman (1981). (c) Suspensory bridging and transfer behaviors in Symphalangus elicit thigh postures throughout the ranges of flexion-extension, adduction-abduction, and rotation. Image modified from Fleagle (1976a). 

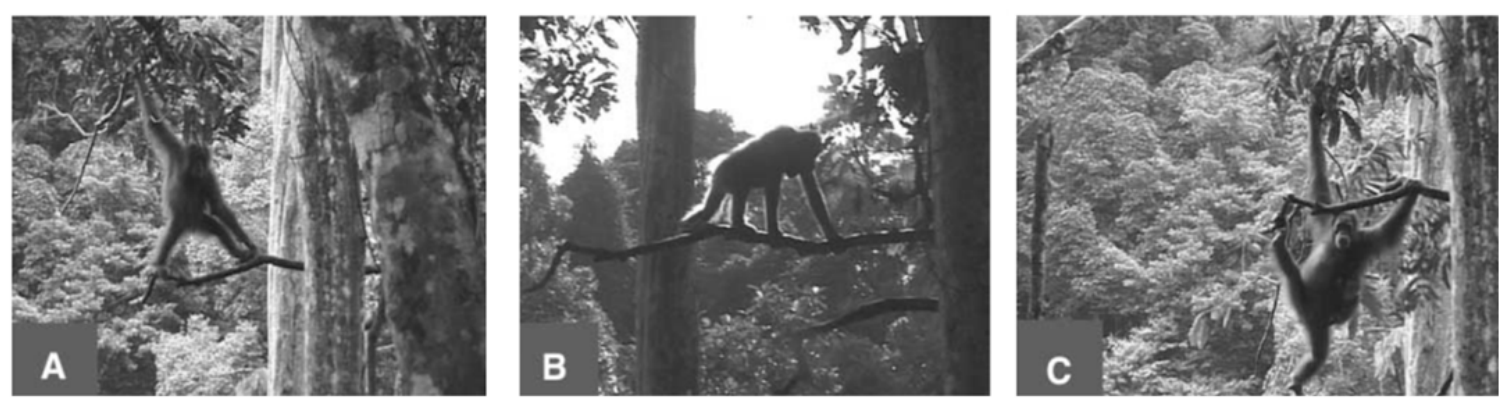

Figure 2.3 Examples of arboreal behaviors used by orangutans

Orangutans are the most acrobatic of the hominids and used virtually all forms of arboreal locomotion, including (a) assisted bipedalism, (b) palmigrade quadrupedalism, and (c) suspension with weight-transfer through both forelimbs and hindlimbs. Image modified from Thorpe et al. (2007b). 


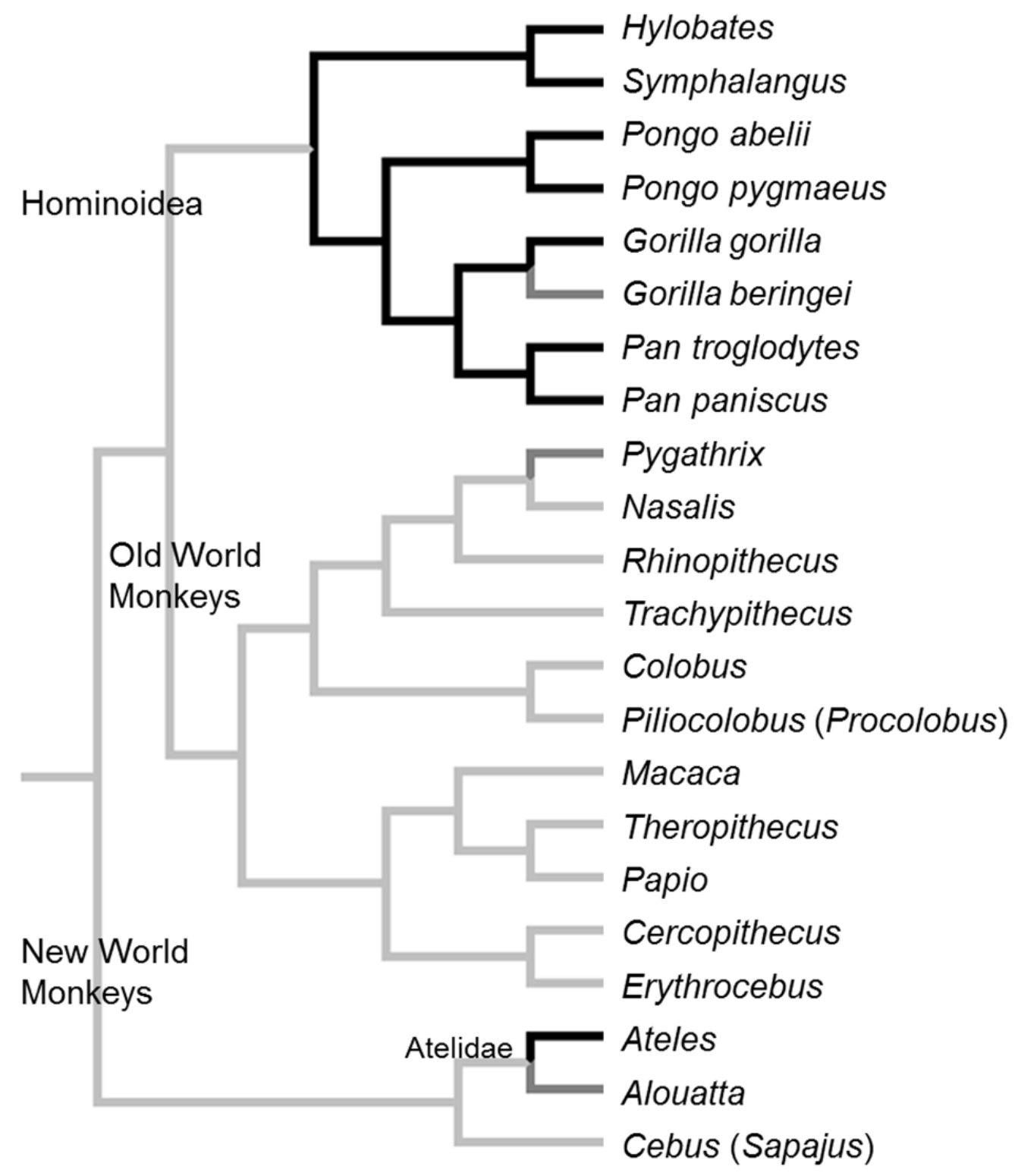

Figure 2.4 Phylogenetic relationships between extant taxa with locomotor classification mapped onto tree

Consensus tree for extant primates based on the 10k Trees Website (Arnold et al. 2010). Locomotor categories from Table 2.2 are mapped onto the taxa (black=suspensory, light grey=non-suspensory, dark gray=indeterminate/unclassified). Extant suspensory primates have evolved from at least two evolutionary events (i.e., hominoids, atelids) and potentially a third (i.e., Pygathrix), providing multiple independent instances in which to evaluate the relationship between suspensory behaviors and morphology. 


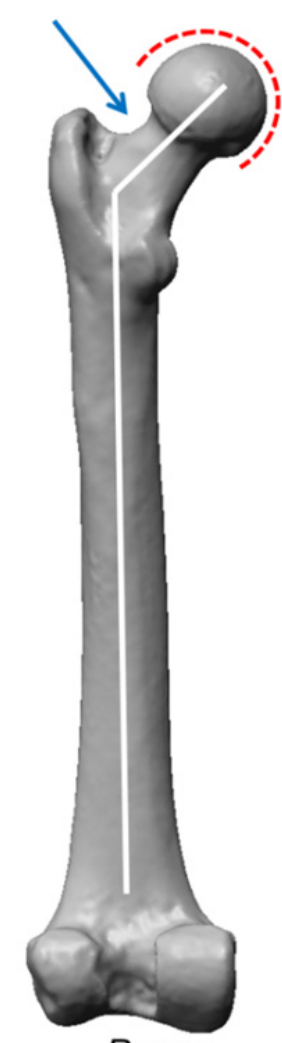

Pongo

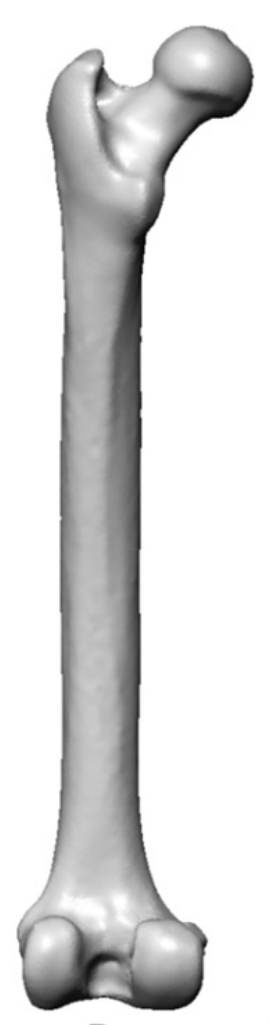

Pan

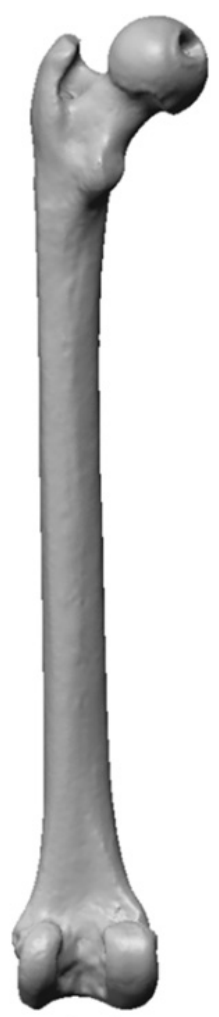

Symphalangus

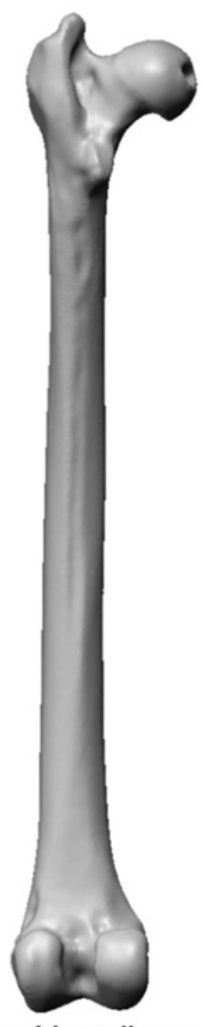

Nasalis

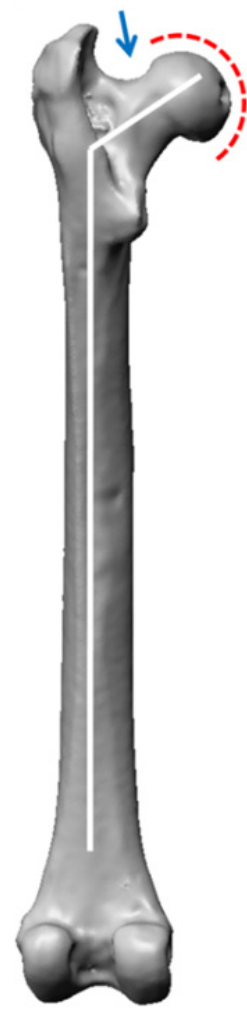

Papio

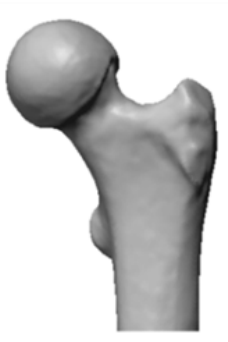

Pongo

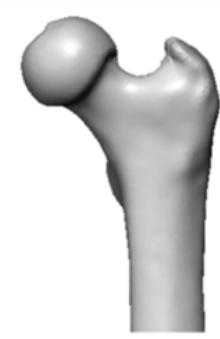

Pan

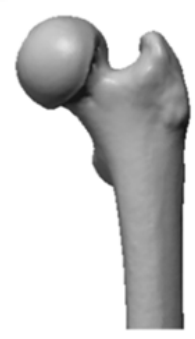

Symphalangus

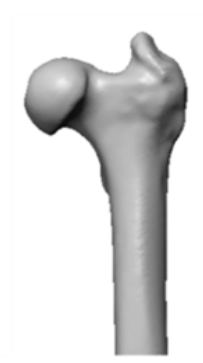

Nasalis

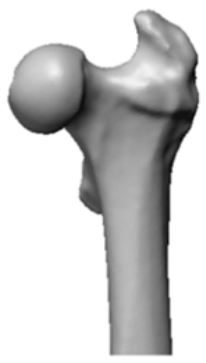

Papio

Figure 2.5 Posterior and anterior views of anthropoid femora

Posterior views of femora (above) and anterior views (below) show variation in femoral morphology. All femora are oriented based on a horizontal orientation of the femoral condyles. Suspensory taxa are described as having a relatively large, spherical femoral head (indicated by dashed line) on a long (arrows) and steeply inclined femoral neck (angle indicated by white lines), freeing the femoral head from the femoral neck in suspensory anthropoids. Additionally, the femoral head is positioned proximal to the greater trochanter in suspensory species whereas the greater trochanter is positioned above the femoral head in non-suspensory taxa. Pongo pygmaeus (USNM 49768 \%), Pan troglodytes (CMNH B1755 +)), Symphalangus syndactylus (ZSM 1905.108 + ), Nasalis larvatus (MCZ $37328 \AA$, mirrored), Papio anubis (USNM 384228 으) are shown. Femora are not to scale. 


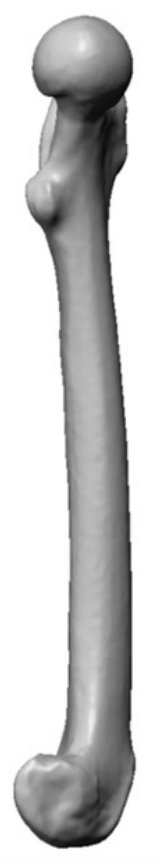

Pongo

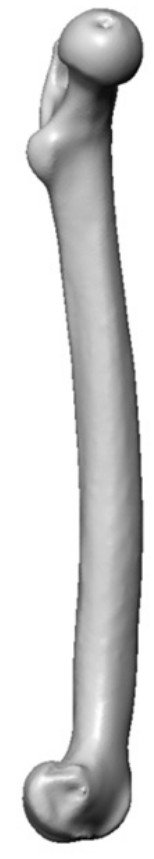

Pan

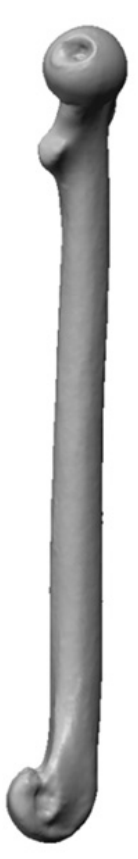

Symphalangus

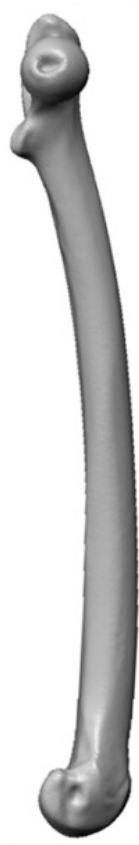

Nasalis
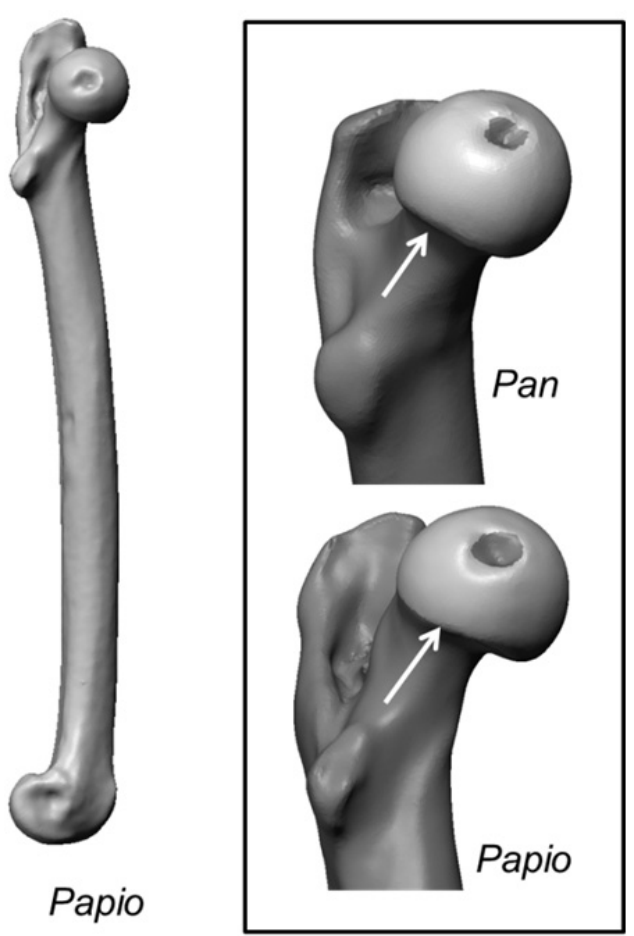

Figure 2.6 Medial views of anthropoid femora, with an emphasis on femoral head articular configuration

The most obvious interspecific differences from medial view are differences in how the femoral head is hafted onto the femoral neck. The femoral head is oriented superiorly in Pongo, anterosuperiorly oriented in Pan, somewhat posteriorly in Symphlanagus, and both cercopithecids have a more medially directed femoral head. The positions of the foveae are also distinct. Pongo lacks a fovea, but Pan and Symphalangus have foveae that are centrally poisoned on the subchondral bone, whereas the two cercopithecids have fovea that are more posteroinferiorly displaced. Because the femoral heads of hominoids are a more complete sphere, the centrally-positioned fovea contributes to a more expansive posteroinferior articular surface. The inset photos of Pan and Papio highlight these differences, with the posteroinferior aspect of the femoral head indicated by a white arrow. Pongo pygmaeus (USNM 49768 q), Pan troglodytes (CMNH B1755 )), Symphalangus syndactylus (ZSM 1905.108 +), Nasalis larvatus (MCZ 37328 ○,, mirrored), Papio anubis (USNM 384228 q) are shown. Femora are not to scale. 


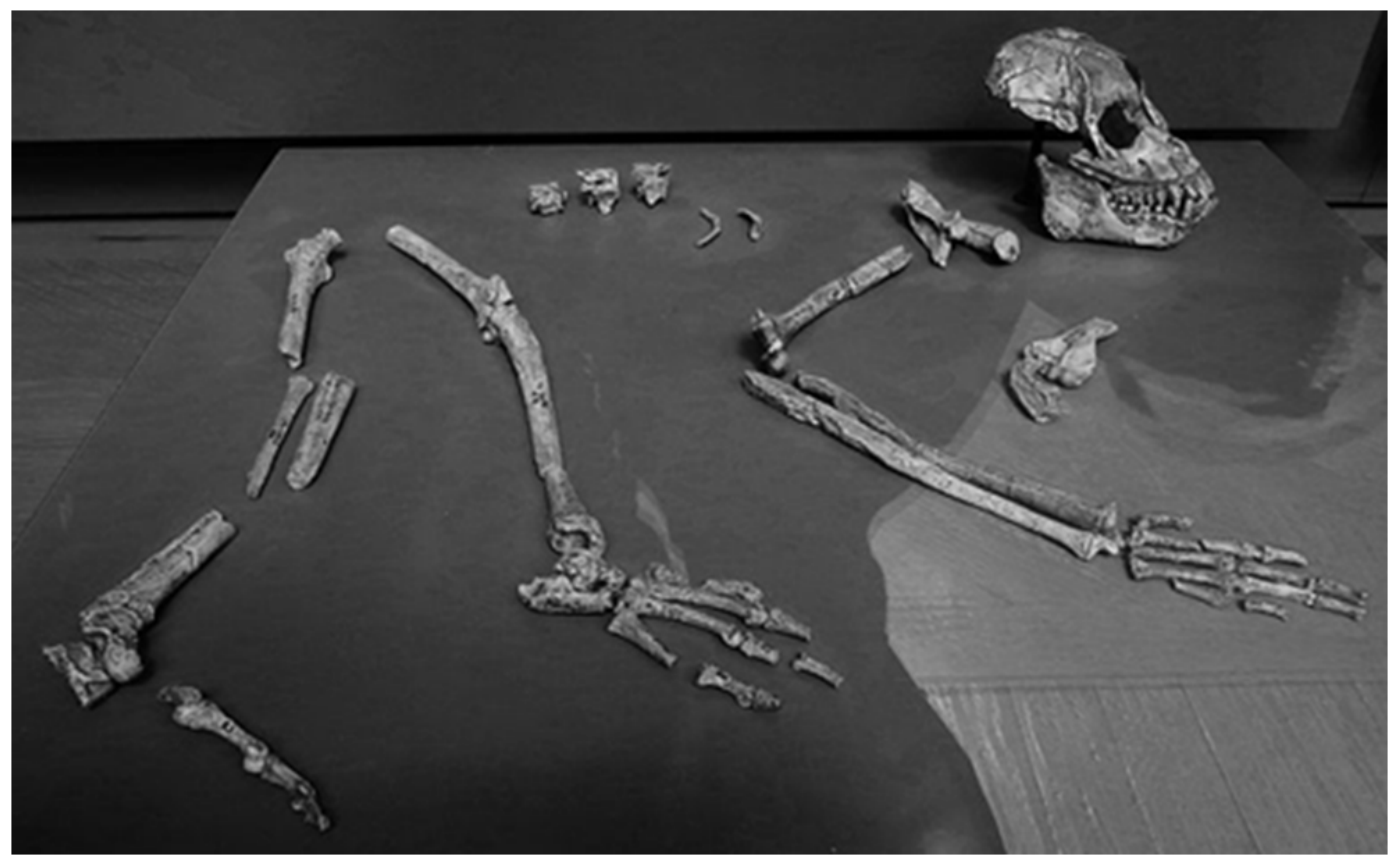

Figure 2.7 An early Miocene hominoid, Proconsul heseloni (KNM-RU 7290 skull and KNM-RU 2036 skeleton), from Rusinga Island, Kenya

Photo from Wikimedia Commons, allowed for reproduction with citation of online location (http://commons.wikimedia.org/wiki/File:Proconsul_nyanzae_skeleton_7.JPG). 


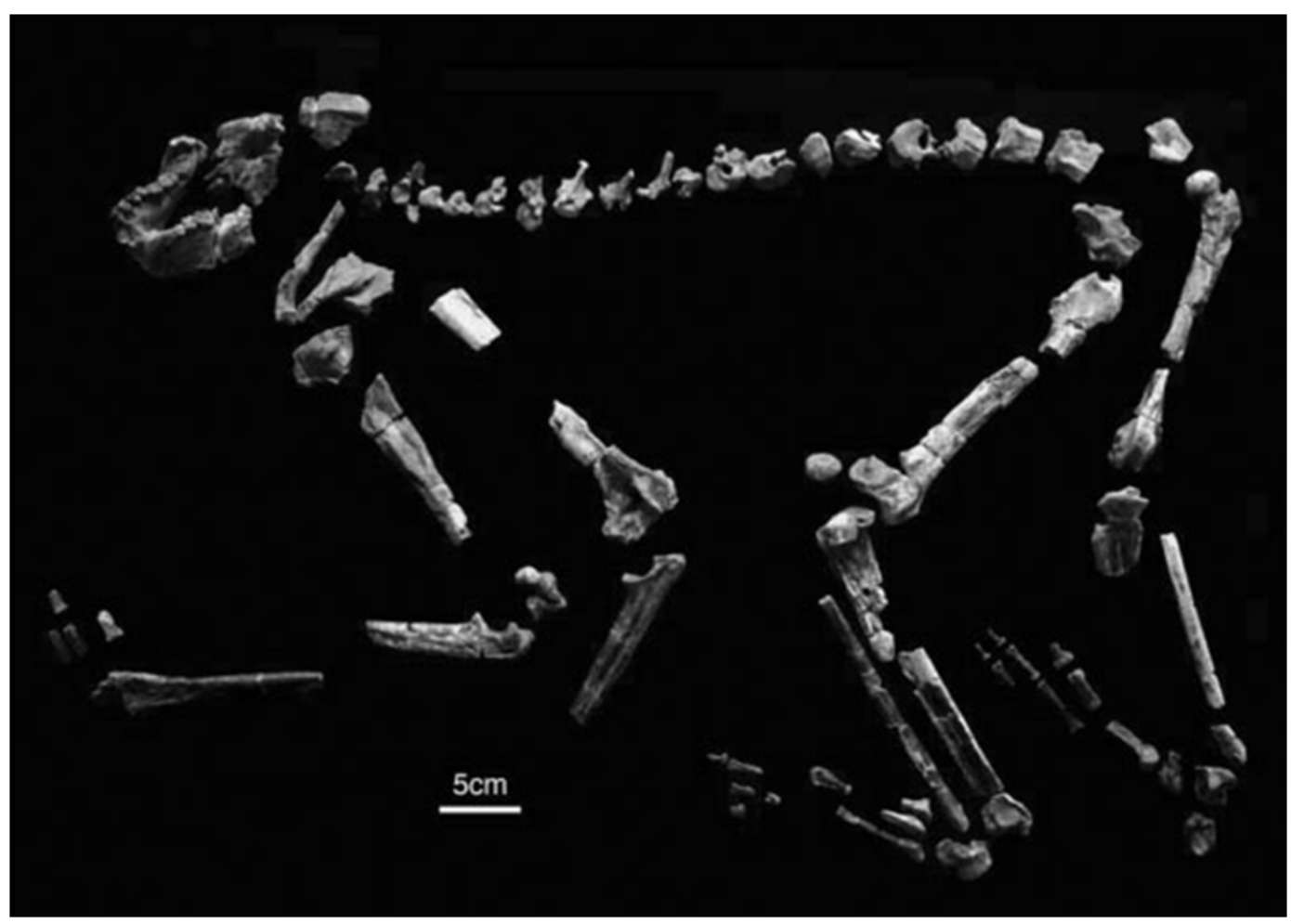

Figure 2.8 A middle Miocene hominoid, Nacholapithecus kerioi (KNM-BG 35250), from Nachola, Kenya

Image modified from Nakatsukasa and Kunimatsu (2009). 

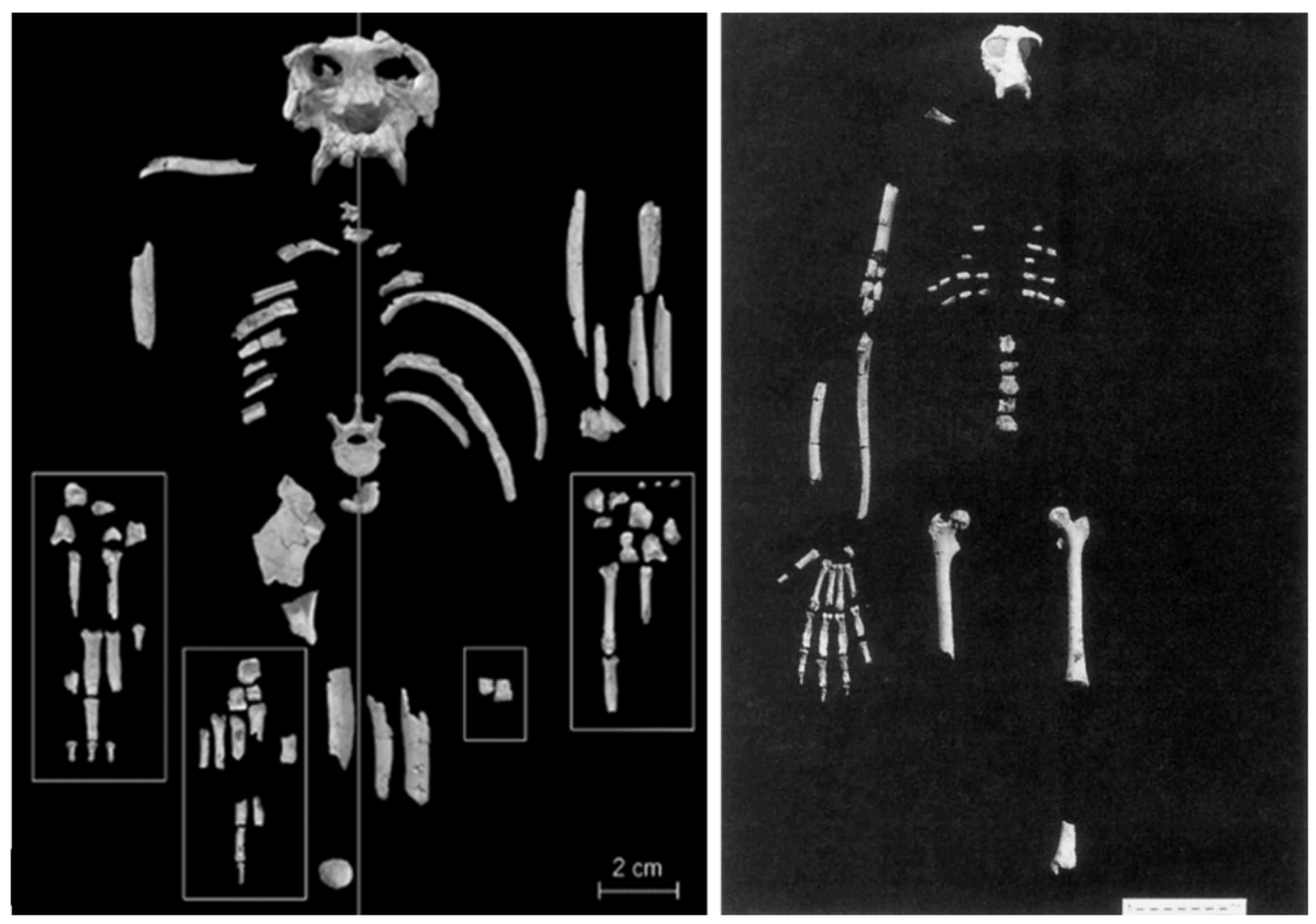

Figure 2.9 Middle to late Miocene hominoids, Pierolapithecus catalaunicus (IPS21350) and Hispanopithecus laietanus (IPS-18800), from the Vallès-Penedès basin in Spain

Pierolapithecus (left) and Hispanopithecus (right) are notably different in their phalangeal morphology and manual proportions, with Hispanopithecus having long and curved proximal phalanges only matched by Pongo and potentially fossil hominoid Rudapithecus. Images modified from Moyà-Solà et al. (2004) and Moyà-Solà and Köhler (1996). 


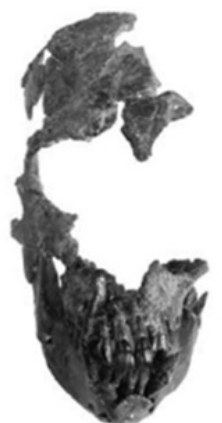

RUD 200 우

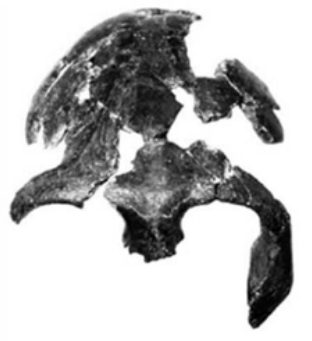

RUD 77 ㅇ

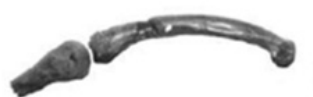

phalanges are long and show

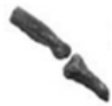

extreme curvature, particularly in the proximal phalanx

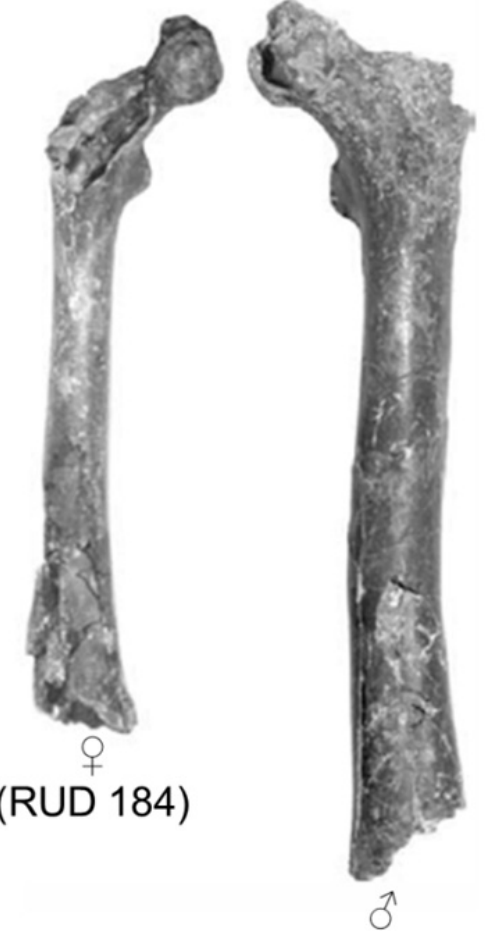

(unassigned specimen)

Figure 2.10 Late Miocene hominoid, Rudapithecus hungaricus, from Rudabánya, Hungary

Rudapithecus hungaricus images are from Begun et al. (2012). 
The hypothesis that arboreal versatility is associated with an increased range of hip joint mobility has influenced functional inferences made about hip morphology when reconstructing the behavior of fossil primates (Walker 1974; e.g., Fleagle 1983; Rose 1983; Ruff 1988; Ward 1993; Ward et al. 1993; MacLatchy 1996; MacLatchy and Bossert 1996; Köhler et al. 2002; Hogervorst et al. 2009). To date, it has never been shown empirically that suspensory anthropoids have higher levels of hip joint mobility than in non-suspensory taxa. Limited data on passive hip joint mobility are available for cercopithecids (DeRousseau et al. 1983; Turnquist 1983; 1985; Turnquist and Kessler 1989) but there are no data from suspensory taxa for comparison. Quantifying range of possible hip joint motion in suspensory and non-suspensory anthropoids would provide empirical data with which to test the hypothesis that suspensory primates have higher levels of hip joint mobility. The goal of this chapter is to evaluate whether suspensory primates actually do have a greater range of motion in the hip joint than non-suspensory primates.

\section{OBJECTIVE}

The hypothesis being tested that there are significant differences in hip joint mobility between suspensory and non-suspensory taxa is tested. Accordingly, the null hypothesis is that there are not significant differences in hip joint mobility between suspensory and nonsuspensory taxa. 


\section{SAMPLE}

The handling and use of animals described herein was approved by the University of Missouri Animal Care and Use Committee (protocol \#6862) and by the animal care committees of the participating zoos and research centers (listed in Table 3.1). In vivo measurements were collected during primate immobilizations for routine veterinary procedures. The use of captive caged animals is not ideal but, because captive animals can be measured in a controlled setting with experienced veterinary professionals in attendance, the use of captive animals was the only realistic and safe method of collecting ROM data. Measurements were collected on live animals rather than cadaveric specimens to avoid changes in soft tissue compliance from decay (e.g., rigor mortis) or chemical embalming (Viidik and Lewin 1966; Tolhurst and Hart 1990; Wilke et al. 1996).

All institutions visited during this study are fully-accredited with the Aquarium and Zoological Association (AZA), the Zoological Association of America (ZAA), and/or the Council on Accreditation of the Assessment and Accreditation of Laboratory Animal Care International (AAALAC). Animal housing was determined by each research location and follow the animal husbandry guidelines for each species. All animals had access to climbing structures within their enclosures, except Macaca individuals. Macaca were housed in cages at the Wisconsin National Primate Research Center.

The subjects used in this study are listed in Table 3.1. Primates categorized as "suspensory" are those species reported to use suspensory behaviors in the wild (Hylobates, Symphalangus, Pongo, Gorilla, Pan, Ateles), whereas "non-suspensory" taxa are those with no reported use of suspension (Colobus, Trachypithecus, Macaca, Papio, Cebus) (see references in Table 1). All animals were weaned and considered 
adults by veterinary staff. However, based on the age of some of the animals (sexspecific means presented in Table 3.2), it is unlikely that they were all skeletally mature at the time of study. Any age effects are considered to be unlikely, as range of motion in cercopithecoids has been shown to be relatively stable and only changing significantly during young (first 18 months) and advanced ages (Turnquist 1983; Turnquist and Kessler 1989). Veterinary records indicate that four of the Ateles included in this study are hybrids of Ateles fusciceps-geoffroyi-robustus and that all Papio are hybrids of Papio hamadryas-anubis-cynocephalus. All animals were selected in consultation with veterinary staff, and animals with a known history of arthritis, lameness, or hindlimb injury were excluded from the study.

\section{METHODS}

\section{Measurements}

The handling and sedation of all subjects was done by licensed veterinarians or veterinary technicians in compliance with the Institutional Animal Care and Use Committee protocols for each research location. Most institutions use an anestheticsedative combination during animal immobilizations. Excluding Cebus apella, all animals were in a deep anesthetic plane at time of study and muscle tone was fully passive. Cebus apella individuals were immobilized using only an oral sedative and ketamine, resulting in some muscle tension during manipulation. Muscle resistance only noticeably occurred in Cebus during measures of hip extension.

Direct measures of (1) flexion, (2) extension, (3) adduction, (4) abduction, (5) internal rotation, and (6) external rotation were measured with each subject laying supine on an examination table. All measurements were collected by the author. In the 
case of male great apes, it became necessary to recruit the assistance of the veterinary staff during some manipulations of the hindlimb. Data were collected using disposable double-armed goniometers and measuring tapes. Measures are based on standard goniometric techniques used in humans (Cole 1971; Norkin and White 1995).

Hip extension and flexion were measured in a parasagittal plane from a lateral view, measuring the angle formed between the femoral diaphysis and the iliac blade (Figure 3.1). Total range of movement from flexion to extension was calculated for each individual by subtracting the value for flexion from the extension value.

A "horizontal" femur posture was then identified for each individual, defined as when the femur approximated a $90^{\circ}$ orientation (perpendicular) to the horizontal surface of the examination table. Although defined based on perpendicular thigh orientation relative to the examination table, this position is termed "horizontal" here because abduction from the horizontal posture matches the "horizontal abduction" described in Stern and Larson (1993) ${ }^{6}$. Measures of adduction, abduction, internal rotation, and external rotation were all measured starting at the "horizontal" posture, which acted as a neutral position to begin measurements across taxa.

The femoral diaphysis was positioned in the horizontal posture and subsequently moved into the maximum positions of adduction and abduction. Abduction and adduction were calculated by measuring the angle formed between the femoral diaphysis and a horizontal plane parallel to the examination table (Figure 3.1) and then adjusting the measures to reflect angles from the midline. In a few overweight apes, it was necessary to measure and convert the inverse angle due to the large amount of

\footnotetext{
6 "Horizontal abduction" of the femur requires additional explanation here. The origins for the term used by Stern and Larson (1993) come from horizontal abduction of the humerus, which brings the arms and forearms horizontal relative to the ground.
} 
abdominal fat that obstructed the placement of the goniometer over the abdomen. Total range of movement from adduction to abduction was calculated for each individual by adding the adduction and abduction values.

Internal and external rotation were measured following the technique used in humans (Cole 1971; Norkin and White 1995), where femoral rotation is calculated based on tibial movement relative to the observer (Figure 3.1). Accordingly, hip rotation measures could be partially influenced by interspecific differences in mediolateral rotation of the tibia on the femoral condyles. Published data on primate range of motion at the knee are lacking, and so this must remain an untested possibility. Internal and external rotation of the femur were measured with the femur oriented in the "horizontal" posture. Total range of movement from internal to external rotation was calculated for each individual by adding the two values together.

Mass $(\mathrm{kg})$ and four linear measures of body size were collected using a digital scale and soft tape measure. All linear measures were rounded to the nearest centimeter $(\mathrm{cm})$ and include overlying soft tissues, making these measures less precise. Linear measures were (1) pelvic breadth measured across the pelvis at the fold between thigh and abdomen, which was identified by flexing the legs, (2) thigh circumference at the level of mid-thigh, (3) thigh length approximated from the anterior hip joint to the anterior center of the patella, and (4) femoral bi-epicondylar breadth measured across the posterior aspect of the knee.

Several additional measures were collected, including (1) maximum hip abduction during flexion, (2) maximum hip abduction during extension, and the (3) distance spanned between the knees during abduction ("abducted knee position”, cm). Abduction during flexion was measured by positioning the leg into the maximally flexed 
position and then maximally abducting the thigh. Likewise, abduction during extension was measured by positioning the leg into the maximally extended position and then maximally abducting the thigh. The abducted knee position was measured by positioning both the thighs into the abducted posture and then measuring the distances between the centers of the palpable patellae. Because the animals sampled in this study were not anesthetized specifically for this project and there were strict time limits for data collection, these three additional measurements could not be collected for all animals and were only measured on a subset of the animals (Pan, Gorilla, Pongo, Symphalangus, Macaca).

\section{Quantitative Analyses}

Quantitative analyses were performed in R (version 3.0.1, R Core Team 2012). Cleveland dot plots were used to visually inspect for differences between groups (Figure 3.2-3.4). Descriptive statistics (mean, standard deviation, and maximum and minimum values) were calculated for both ROM variables and body size variables. Significant differences in ROM between suspensory and non-suspensory anthropoids were tested by means of one-way analysis of variance (ANOVA), with significance assessed at $p \leq 0.05$.

In certain measures (e.g., maximum flexion), ANOVA detected significant differences between locomotor groups when visual inspection of the descriptive plots suggested substantial overlap between the broad locomotor groups. In order to provide a finer resolution of the significant ANOVA results, post-hoc Tukey's honestly significant difference test (Tukey's HSD) was used as conservative method of multiple comparisons between species. Tukey's HSD is appropriate for testing groups of unequal sample 
sizes. Male and female data were grouped for ANOVA and Tukey's HSD comparisons due to the small sample sizes for some species.

\section{$\underline{\text { Phylogenetic regression and ordinary least squares regression }}$}

Range of motion variables were tested for a significant relationship with logtransformed body mass $(\mathrm{kg})$ using non-parametric Spearman's rank correlations. A significant correlation $(p \leq 0.05)$ indicates a relationship with body mass.

The ROM variables were then examined versus log-transformed body mass between locomotor groups, with slopes and intercepts of the locomotor-specific regressions tested for significant differences. Allometric slopes were estimated using a phylogenetic generalized least squares (PGLS) and an ordinary least squares (OLS) ${ }^{7}$ approach. PGLS models are included in this study because comparative data violate the assumption of non-independence of data points in OLS (Felsenstein 1985; Grafen 1989). PGLS and independent contrasts are statistically equivalent techniques to detect correlated evolution (Garland Jr and Ives 2000; Rohlf 2001) but PGLS has more flexibility in modeling the process of evolution (i.e., independent contrasts assumes a genetic drift model) and uses raw data rather than contrasts, allowing a more intuitive assessment of the results (e.g., intercepts and standard errors can be directly extracted) (review in Nunn 2011). Some workers also argue that PGLS is more reliable than independent contrasts (Martins et al. 2002; Rohlf 2006).

Within the caper package of $\mathrm{R}$ (Orme et al. 2012), regressions are weighted by incorporating phylogenetic distances between taxa, calculated from branch lengths of a phylogeny. Phylogenetic distances are introduced as the error term via the variance-

\footnotetext{
${ }^{7}$ OLS was appropriate over standardized major axis regression (SMA) because ROM values were regressed on body size, which was measured precisely using a digital scale and should therefore introduce virtually no error on the $x$-axis.
} 
covariance matrix. The variance-covariance matrix represents tree structure with the matrix diagonal representing the distance (i.e., length in time) from root to tip for each species, with the off-diagonals containing the distances between pairs of species, as measured from the root of the last common ancestor to the tip (Freckleton et al. 2002). The variance-covariance matrix is then scaled by Pagel's lambda $(\lambda)$ parameter (Pagel 1999; Freckleton et al. 2002), a measure of the dependence of a trait on a specified tree. Pagel's $\lambda$ was estimated from the data via a maximum likelihood approach. Pagel's $\lambda$ typically ranges from $0-1$, with data showing increasing phylogenetic dependence as $\lambda$ approaches 1 . When $\lambda$ is estimated to be equal to 0 , the PGLS regression should converge on the OLS model.

The phylogenetic tree used for PGLS analyses (Appendix A) was a consensus tree downloaded from the 10k Tree Project (Arnold et al. 2010). Papio cynocephalus and Ateles geoffroyi were used for Papio hamadryas-anubis-cynocephalus and Ateles fusciceps-geoffroyi-robustus hybrids. PGLS requires a single value for each tip in the phylogeny and, because averaging male and female data can result in unrealistic values for sexually dimorphic species, only female data points were used. It should therefore be emphasized that the PGLS linear modeling used here differs from traditional OLS in two key ways. First, OLS regression by default assumes no phylogenetic relationship between the data points (e.g., independent data points) whereas PGLS incorporates information about phylogenetic distances. Second, because PGLS regression requires that a single value is assigned to each terminal tip in the tree, the PGLS (female means only) regressions have half the number of data points of OLS (male and female means). 


\section{RESULTS}

\section{ANOVA results}

Sex-specific descriptive statistics for range of motion are provided in Tables 3.3-3.5, with additional size measures from the sample reported in Table 3.6. Data are also shown in descriptive plots in Figures 3.2-3.5. This study found significantly larger ranges of motion in suspensory taxa for maximum measures extension $(p<0.001)$, abduction $(p<0.001)$, external rotation $(p<0.001)$, range of abduction-adduction $(p<0.001)$, and range of internal-external rotation $(p<0.001)$. Non-suspensory taxa had significantly higher mobility in flexion $(p<0.001)$ and internal rotation $(p=0.03)$. Adduction $(p=0.73)$ and range of flexion-extension $(p=0.63)$ were not significantly different between suspensory and non-suspensory taxa. Suspensory anthropoids also have significantly larger abduction during flexion $(p<0.001)$, abduction during extension $(p<0.001)$, and abducted knee positions ( $p<0.001$ ) (descriptive statistics presented in Table 3.10).

\section{Tukey's HSD pairwise comparisons}

Although there are statistically significant differences by locomotor group, there is substantial overlap between the two groups in degree of flexion, extension, and internal rotation (Figure 3.2 and 3.4). Tukey's HSD pairwise comparisons provide a finer resolution of the significant differences detected with ANOVA by identifying which pairs of species are significantly different from one another (Tables 3.7-3.9, 3.11). Tukey's HSD comparisons found more comparisons between suspensory and non-suspensory taxa to be significant at $(p \leq 0.05)$ than within a single locomotor group (e.g., suspensory vs. suspensory, non-suspensory vs. non-suspensory). However, many comparisons within the same locomotor group were also significant. 


\section{$\underline{\text { Spearman correlations and regressions }}$}

Flexion, range of flexion-extension, and external rotation were found to have significant Spearman rank correlations with body mass (Table 3.12). External rotation increases with increasing body size, although flexion and range of flexion-extension decrease with increasing body size. It is important to note that, although flexion has a significant positive Spearman rank correlation value $(r h o=0.68, p=0.02)$ indicates a positive scaling relationship between numerical values for flexion and body size, higher numerical values for flexion indicate a reduced range of motion (refer to Figure 3.1). The result therefore indicates that range of motion in flexion is decreasing with body size, which is clarified by the significant negative relationship between range of flexionextension and body mass.

Female suspensory and non-suspensory ROM data were regressed against body size using phylogenetic regression (Table 3.13). The slopes and intercepts were not significantly different between locomotor groups for any variable except internal rotation ( $\mathrm{p}_{\text {slope }}$ and $\mathrm{p}_{\text {intercept }}$, Table 3.13). Flexion and range of flexion-extension, had non-significant differences in slopes and intercepts between the two locomotor groups. This result suggests that, although flexion and range of flexion-extension have a significant correlation with body size, the scaling properties are not different between locomotor groups. Interestingly, the slopes for suspensory and non-suspensory primates denotes opposite scaling relationships for internal and external rotation. With increasing mass in suspensory primates, internal rotation decreases and external rotation increases, whereas internal rotation increases and external rotation decreases with increasing mass in non-suspensory primates. However, the differences in external rotation are not statistically different between locomotor groups. The lambda values 
used in the phylogenetic regressions were estimated to be zero using a maximumlikelihood approach, suggesting little phylogenetic signal in the data. The allometric patterns found using female-specific data in phylogenetic regression are similar to those produced using male and female data in OLS (see Table 3.12), which is not surprising given that there is not phylogenetic signal present in the data.

\section{DISCUSSION}

This study finds significantly larger abduction and external rotation in suspensory taxa. It has previously been hypothesized that limb abduction is a critical movement during climbing and suspensory behaviors (Grand 1972; Jenkins 1974; Grand 1984; Cartmill 1985; Larson 1993; Crompton et al. 2008; Schmidt and Krause 2011), and these results support an association between hip abduction and suspensory behaviors. However, not all measures of joint mobility are necessarily increased in suspensory species relative to the non-suspensory species. Levels of adduction are not significantly different, and non-suspensory primates are actually capable of larger amounts of flexion and internal rotation.

Because hip mobility shows differences in mobility that track locomotor behavior, hip joint mobility seems to be a better indicator of habitual limb postures used during locomotion than glenohumeral joint mobility. Recent work has shown that the hominoid glenohumeral joint does not actually produce a greater range of circumduction than other primates (Chan 2007a; b), with few differences in passive range of motion observed between suspensory hominoids and non-suspensory cercopithecids (Chan 2008). One potential reason that Chan's work may not have detected significant differences between locomotor groups relates to the structure of the anthropoid shoulder itself. The humeral head is surrounded by soft tissues with only a small receiving 
articular glenoid on the scapula, whereas the femoral head is more deeply received by the cup-like acetabulum and surrounding soft tissues. Moreover, the humerus is indirectly attached to the trunk by the scapula via the clavicle, with movements possible at the acromioclavicular and sternoclavicular articulations. These secondary articulations might provide some additional shoulder mobility that cannot be strictly differentiated from glenohumeral joint movements in a goniometric study. In contrast, the femoral head directly articulates with the trunk (i.e., in the acetabulum of the bony pelvis) and femoral movements are therefore easier to isolate.

Some differences detected between suspensory and non-suspensory taxa using ANOVA are potentially influenced by the values for Pan and Macaca, necessitating pairwise comparisons between all species. Overall, pairwise comparisons between suspensory and non-suspensory taxa yielded a higher number of significant differences than within a single locomotor group. Not surprisingly, highly suspensory Pongo was found to have significantly larger abduction and external rotation than all non-suspensory taxa. Because males and females were grouped together for the (species-specific) pairwise comparisons due to small sample sizes, some male and female differences within a species might be obscured by these comparisons. In particular, the Gorilla intraspecific means for maximum flexion and ranges of flexion-extension differ by more than $30^{\circ}$ (Tables 3.3-3.5). Therefore some caution should be used when interpreting the results for Gorilla, as there may actually be significant sex-specific differences in degree of flexion or range of flexion-extension that are not detected when using the species mean value. A larger sample of gorillas might clarify whether significant inter- and intraspecific differences exist, but this cannot currently be determined with the sample size in this study. 
Body size does not influence range of hip mobility overall (Table 3.13, supplemented by OLS in Table 3.12). There is a significant correlation $\left(p_{\text {rho }}\right)$ between mass and flexion but the PGLS slopes and intercepts for flexion do not differ significantly by locomotor group. Descriptive plots of the data (Figure 3.2) show that all taxa except Pongo and some Gorilla have similar ranges of flexion. Thus, for flexion, it seems that grouping taxa into "suspensory" and "non-suspensory" categories for regression might mask some of the allometric differences. Moreover, although there is a non-significant correlation $\left(\mathrm{p}_{\mathrm{rho}}\right)$ between body mass and internal rotation, the suspensory and nonsuspensory locomotor groups have significantly different scaling relationships. However, when the raw values for internal rotation are examined in the descriptive plots (Figure 3.4), it is apparent that there is substantial overlap between locomotor groups. Because there is little evidence for a relationship between range of motion variables and body mass, this supports the hypothesis that differences in mobility are related to locomotor behavior and are not strictly a consequence of allometry.

Distance between the knees during abduction (Table 3.10), a proxy for the hindlimb spatial envelope (see Chapter 2), was evaluated as a potential metric of locomotor adaptation within anthropoids. Although the abducted knee position is largely a consequence of body size, it is also a factor of hip mobility. For instance, Symphalangus syndactylus has a span at the knee that is approximately double similarly-sized male Macaca mulatta. Interestingly, Gorilla has a significantly smaller range of angular abduction than Pan and Pongo yet has an equivalent distance between the knees during abduction (Tables 3.10-3.11), suggesting that the functional outcome of hip mobility (abducted knee position) is influenced by both body size and hip 
morphology. The relationship between range of motion, morphological variation in the hip, and the spatial envelope warrants further consideration.

Additionally, the influence of external rotation in locomotor abilities should be considered. According to Stern and Larson (1993), when the hip is flexed, external rotation of the hip simultaneously occurs in order to achieve an abducted thigh posture. Specifically, Stern and Larson (1993) state that when the femur is flexed to $90^{\circ}$ and then abducted (as in this study), $90^{\circ}$ of lateral femoral rotation simultaneously occurs. The abducted position measured here, which begins at the "horizontal" posture (defined in Materials and Methods), is near the middle of the flexion-extension range for most species and some conjunct external rotation would therefore be expected to accompany abduction. Because external rotation occurred in unison with abduction, it was not possible here to quantify how much external rotation contributed to abduction ability. However, this study confirmed that non-suspensory taxa achieved abducted femur positions primarily by lateral excursions relative to the midline, whereas abducted femoral postures in suspensory taxa were accompanied by a high level of conjunct external rotation (Figure 3.6). Thus, lateral rotational ability at the hip appears to be a key determinant of abduction ability which has been largely neglected. Because this study suggests that an ability to highly abduct the thigh in suspensory primates is conferred with a concomitant ability to externally rotate the femur, hypotheses regarding the evolution of hominoid locomotor behaviors should consider the use of laterally rotated hip postures in addition to abduction.

The joint modeling work by MacLatchy $(1995 ; 1996)$ predicted that range of abduction would discriminate locomotor adaptation, specifically that "differences in abduction during extension may be an indicator of differential ability to assume versatile 
climbing postures, while abduction during flexion may be a more general indicator of arboreality" (MacLatchy 1996, p. 471-472). Mean abduction in flexed and extended postures was found to be within $10^{\circ}$ of the mean for abduction from the horizontal posture in all hominoids except male gorillas (Tables 3.4 and 3.10). With the exception of male gorillas, which displayed a substantially reduced degree of abduction in an extended posture (mean $48^{\circ}$ from horizontal vs. $9^{\circ}$ in extension), range of abduction in hominoids is similar throughout the flexion-extension range. As hypothesized by MacLatchy (1996), the ability to abduct the thigh in extended positions is associated with high levels of arboreal versatility (e.g., suspension), although large abduction ability in any position appears to be associated with arboreal versatility. However, these data show that hip abduction during flexion is probably not a strong indicator of arboreality in general, as Macaca fascicularis is a skilled arboreal quadruped (Rodman 1979) with a low range of abduction possible during flexed hip postures.

There are certain considerations for the baseline values reported in this study. First, this study relies on an opportunistic sample of captive primates. The animals sampled almost certainly did not use their hindlimbs exactly as they would in the wild, and may potentially have ranges of motion, morphologies, and body masses that are different from wild counterparts. Past studies have typically found captive animals to have a greater range of motion rather than free-ranging individuals (Turnquist 1983; 1985). Relying on captive animals may increase the variation of the sample, potentially reducing the likelihood of detecting significant differences between groups. However, significant differences were detected between locomotor groups, and the range of motion data collected for cercopithecids is consistent with all other published ROM data (DeRousseau et al. 1983; Turnquist 1983; 1985; Turnquist and Kessler 1989). The degree of hip abduction in this study is consistent with DeRousseau et al. (1983)'s 
findings for Macaca mulatta (mean $\sim 30^{\circ}$ to midline, versus $25^{\circ}$ here), and the mean values for adduction are identical in both studies (mean $36^{\circ}$ ). The range of hip flexion documented here in cercopithecids is also consistent with previous measures of hip flexion provided by Turnquist and Kessler (1989), who reported low maximum hip flexion values in $M$. mulatta (approximately $11-49^{\circ}$ versus $28-41^{\circ}$ here).

Second, passive range of motion is the maximum range of motion allowed at the joint, and it is worth noting that a passive range might differ substantially from the range normally employed by a living animal. Unfortunately, there are few data available to compare the key measures of passive abduction and external rotation due to difficulties measuring these with traditional kinematic techniques. The passive data presented here are consistent with Isler's (2005) data on hominoid active hip abduction and range of adduction-abduction during vertical climbing. Isler's (2005) values for Hylobates indicate that they use a substantially lower range of abducted hip postures during vertical climbing than they are capable of passively. Isler (2005) also finds a single male gorilla showing an increased ROM relative to a single female gorilla, which is a pattern opposite of what was observed in this study. It remains unknown whether the Hylobates and Gorilla values differ due to sampling or perhaps real differences in active ROM.

Third, questions remain as to what determines hip joint range of motion. Multiple morphologies of the pelvis and femur have been related to hip mobility (e.g., Napier and Walker 1967; Schultz 1969; Walker 1974; Stern and Susman 1983; Fleagle and Anapol 1992; Ward 1993; Ward et al. 1993; MacLatchy 1995; 1996; MacLatchy and Bossert 1996; MacLatchy 1998). For instance, a longer femoral neck length has been related to mobility at the primate hip joint by increasing the distance between the bony pelvis and the greater trochanter, allowing for more abduction potential (Fleagle and Meldrum 
1988). A lower greater trochanter should enable more abduction before impingement of bony or soft tissues occurs (MacLatchy 1995; 1996), and this effect could even be amplified by having a high neck-shaft angle, which would further position the femoral head above the greater trochanter. The distribution of the subchondral bone on the femoral head (Jenkins 1972; Fleagle 1976b; Jenkins and Camazine 1977; Ward et al. 1993; MacLatchy 1996; MacLatchy and Bossert 1996) and potentially femoral head size (Ruff 1988) are hypothesized to relate to hip mobility. The geometry of the femur itself might be especially influential in measuring abduction-adduction, as higher neck-shaft angles will increase the angle of the thigh relative to the midline for any given hip position. In order to test the relationship between morphologies and ROM, future studies should attempt to directly quantify morphologies using x-ray or CT data from the same individuals that ROM data is collected from.

Joint pathologies such as osteoarthritis reduce joint mobility (e.g., Steultjens et al. 2000), limiting normal joint gliding movements by atypical bone growth, fusions, or joint erosions. Naturally-occurring osteoarthritis and other inflammatory diseases of the joints are observed in catarrhines (DeRousseau 1988; Rothschild and Woods 1989; 1992b; 1996; Rothschild 2005; Rothschild and Ruhli 2005), although incidences of arthritis specifically at the anthropoid hip joint are either rare or under-reported (Schultz 1944; Bramblett 1967; Rothschild and Woods 1992a; 1996; DeGusta and Milton 1998; Nakai 2003). In healthy joints, such as those sampled in this study, species-specific differences in observed ranges of motion are likely to originate from bony or soft tissue features.

However, in normally functioning joints, range of motion is determined primarily by soft tissue structures surrounding the joint itself, such as ligament, tendon and 
passive muscle tension (Kapandji 1970; Wright and Radin 1993; Levangie and Norkin 2005; McGinnis 2005; Standing and Gray 2008; Safran et al. 2012). A number of soft tissues probably influence the movements at the hip joint, including the ligamentum teres femoris, the acetabular labrum, the hip joint capsule and associated ligaments, and surrounding (passive) hip musculature. Interspecific variation in soft tissue attachments and/or size can contribute to differences in mobility observed between species, limiting movement by differences in length, orientation, and even by physical obstruction.

Little is known about the composition, material properties, variation, size/strength of the deep hip joint soft tissue structures in nonhuman anthropoids (Sonntag 1923; 1924; Howell and Straus 1933; Raven 1950; von Klaus Uhlmann 1968; Sigmon 1969; 1974; Payne 2001; Payne et al. 2006a; b) and how these factors might relate to hip mobility. The joint capsule, particularly in reference to the three capsular ligaments, is thought to be one of the strongest soft tissue limits on femoral head movement within the acetabulum (Myers et al. 2011; Smith et al. 2011; Smith et al. 2012). The limited data on the non-human primate hip capsule suggest that the chimpanzee and orangutan capsule is loose with indistinct capsular ligaments (Sonntag 1923; 1924), allowing more mobility than that of cercopithecids, which have a broad, thick iliofemoral ligament (Keith 1894; Howell and Straus 1933; Walji 1988). Several studies have found a link between hip stability and ligamentum teres integrity (Chen et al. 1996; Demange et al. 2007; Wenger et al. 2007; Dodds et al. 2008; Bardakos and Villar 2009; Martin et al. 2011). If the ligamentum teres actually is mechanically limiting joint movements, it appears to do so when the femur is in a position of combined adduction, flexion, and external rotation (Dodds et al. 2008), and so it is not necessarily an explanation for the limited abduction in cercopithecoids here. Likewise, the acetabular labrum limits movements of the 
femoral head within the acetabulum by deepening the acetabular socket and by increasing the negative intra-articular joint pressure (Crawford et al. 2007; Myers et al. 2011; Smith et al. 2011; Safran et al. 2012), but no data are published on labrum size or structure in nonhuman anthropoids.

Muscular structure can also limit active (voluntary) range of motion through mechanoreceptor feedback, as well as limit motion passively by physically obstructing movement at the joint or through tension in the antagonistic muscles and associated tendons (Brinckmann et al. 2002b; Levangie and Norkin 2005). Although anthropoid hip muscle attachments are generally similar (Anapol and Barry 1996; Thorpe et al. 1999; Payne et al. 2006a; b; Myatt et al. 2011), the mechanical arrangement of the muscles around the joint differs due to skeletal structure. For instance, great ape and hylobatid gluteal, hip adductor, quadricep, and hamstring muscles have smaller physiological cross section areas and longer fascicles compared to Homo sapiens (Payne 2001; Payne et al. 2006b; a; Channon et al. 2009), which would allow a higher range of motion at the hip joint (Payne 2001). Interspecific differences in muscle attachments, orientations, size, extensibility/stretch and tone could therefore affect range of passive movement at the hip joint.

It is difficult to speculate as to what soft tissue structures specifically restricted or enabled the different ranges of motion observed in this study. As was previously noted, hip rotation as measured here could potentially be increased by a more mobile knee joint, and so this may have factored into the results. However, manual manipulations of the hip joint through its range of circumduction implicated strong, deep ligamentous hip structures in limiting both abduction and external rotation in the non-suspensory taxa. This inference is made by the author because the limits were not bony and there was no 
observable muscle tension during maximum positions of the limb, except perhaps in hip extension. As previously mentioned (Chapter 2), there is some evidence that cercopithecids have stronger, more distinct ligaments in the hip joint capsule compared to orangutans and chimpanzees (Sonntag 1923; 1924; Howell and Straus 1933). Given that the superior band of the iliofemoral on the anterior aspect of the capsule limits external rotation in humans, it is possible that the iliofemoral ligament plays an important role in limiting external rotation (and in turn, abduction) in cercopithecids. This is supported by the histological findings on the vervet hip joint capsule by Walji (1988) described the anterior and superior portions of the capsule as having a strong collagenous structure which would limit excessive range of motion. Detailed comparative dissection and histological work is necessary to evaluate how interspecific differences in size, strength, and/or configuration of soft tissue structures relate to hip joint mobility.

\section{CONCLUSIONS}

For the first time, in vivo data on hip joint range of motion in hominoids and Ateles are presented and quantitatively compared to cercopithecids and Cebus. The data for cercopithecids is comparable to other studies. The null hypothesis that there are no significant differences in hip joint mobility between suspensory and nonsuspensory taxa can be rejected, with significant increases in suspensory anthropoids in range of external hip rotation and hip abduction. It is hypothesized here that the ability to differentially abduct the thigh is facilitated by an increased range of external rotation at the hip joint. Moreover, the analyses show that range of motion is not strictly a consequence of body size but does indicate that higher mobility increases the "spatial envelope" for a given body size. Limits on external rotation and abduction in non- 
suspensory taxa are soft-tissue constraints and it is proposed here that range of motion was probably primarily constrained by strong capsular ligaments of the hip joint. Formal characterization of bony and soft tissue structures in anthropoids is needed to clarify why non-suspensory species have a reduced range of abduction and external rotation. 


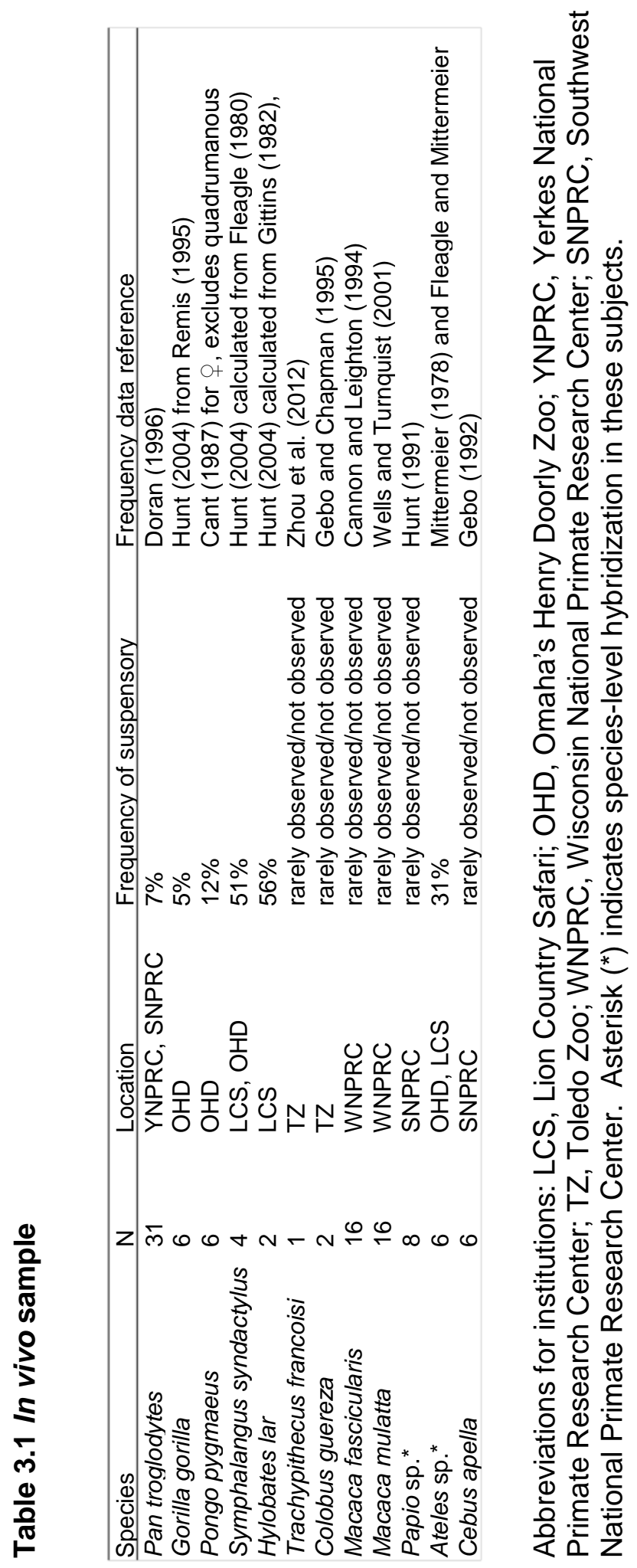


Table 3.2 Sex-specific descriptive statistics for age and body size

\begin{tabular}{|c|c|c|c|c|c|c|}
\hline \multirow[b]{2}{*}{ Species } & \multirow[b]{2}{*}{ Sex } & \multirow[b]{2}{*}{$\mathrm{n}$} & \multicolumn{2}{|l|}{ Age (years) } & \multicolumn{2}{|l|}{ Mass $(\mathrm{kg})$} \\
\hline & & & Mean \pm SD & Max, Min & Mean \pm SD & Max, Min \\
\hline \multirow{2}{*}{ Pan troglodytes } & $F$ & 13 & $20.2 \pm 8.4$ & $31.0,5.6$ & $54.1 \pm 14.3$ & $79.5,30.6$ \\
\hline & $M$ & 18 & $21.1 \pm 5.3$ & $31.9,14.0$ & $67.4 \pm 7.3$ & $79.3,56.8$ \\
\hline \multirow[t]{2}{*}{ Gorilla gorilla } & $\mathrm{F}$ & 2 & $13.3 \pm 5.6$ & $17.2,9.4$ & $70.8 \pm 5.3$ & $74.5,67.0$ \\
\hline & $M$ & 4 & $18.3 \pm 6.6$ & $28.0,13.2$ & $198.8 \pm 19.2$ & $225.0,179.0$ \\
\hline \multirow[t]{2}{*}{ Pongo pygmaeus } & $\mathrm{F}$ & 3 & $10.5 \pm 2.2$ & $12.0,7.9$ & $47.6 \pm 14.5$ & $62.1,33.1$ \\
\hline & $M$ & 3 & $12.8 \pm 7.5$ & $20.2,5.1$ & $74.4 \pm 64.9$ & $148.3,27.2$ \\
\hline \multirow[t]{2}{*}{ Symphalangus syndactylus } & $\mathrm{F}$ & 2 & $17.4 \pm 12.1$ & $25.9,8.8$ & $13.6 \pm 6.2$ & $17.9,9.2$ \\
\hline & $M$ & 2 & $17.6 \pm 8.1$ & $23.3,11.9$ & $14.3 \pm 4.8$ & $17.7,10.9$ \\
\hline \multirow[t]{2}{*}{ Hylobates lar } & $\mathrm{F}$ & 1 & 23.5 & N/A & 6.5 & $\mathrm{~N} / \mathrm{A}$ \\
\hline & M & 1 & 23.9 & $\mathrm{~N} / \mathrm{A}$ & 10.7 & N/A \\
\hline \multirow[t]{2}{*}{ Colobus guereza } & $\mathrm{F}$ & 1 & 2.9 & $\mathrm{~N} / \mathrm{A}$ & 7.0 & $N / A$ \\
\hline & $M$ & 1 & 4.5 & $N / A$ & 13.2 & $N / A$ \\
\hline Trachypithecus francoisi & M & 1 & 3.0 & $N / A$ & 7.0 & $N / A$ \\
\hline \multirow[t]{2}{*}{ Macaca fascicularis } & $\mathrm{F}$ & 8 & $9.4 \pm 1.4$ & $12.1,7.7$ & $4.8 \pm 0.9$ & $6.0,3.5$ \\
\hline & M & 8 & $7.7 \pm 0.5$ & $8.6,7.0$ & $6.9 \pm 1.1$ & $9.2,6.1$ \\
\hline \multirow[t]{2}{*}{ Macaca mulatta } & $\mathrm{F}$ & 8 & $9.1 \pm 0.3$ & $9.5,8.6$ & $8.3 \pm 1.3$ & $9.8,7.0$ \\
\hline & M & 8 & $9.0 \pm 0.2$ & $9.3,8.8$ & $12.1 \pm 1.9$ & $14.1,9.1$ \\
\hline \multirow[t]{2}{*}{ Papio sp. } & $\mathrm{F}$ & 3 & $9.6 \pm 0.8$ & $10.4,8.9$ & $17.7 \pm 1.1$ & $18.8,16.6$ \\
\hline & M & 5 & $10.5 \pm 0.5$ & $11.4,10.1$ & $28.4 \pm 1.7$ & $31.0,26.5$ \\
\hline \multirow[t]{2}{*}{ Ateles sp. } & $\mathrm{F}$ & 3 & $18.0 \pm 10.5$ & $27.9,7.0$ & $9.3 \pm 1.8$ & $11.2,7.7$ \\
\hline & M & 3 & $18.7 \pm 15.8$ & $37.0,9.2$ & $8.2 \pm 0.2$ & $8.3,7.9$ \\
\hline \multirow[t]{2}{*}{ Cebus apella } & $\mathrm{F}$ & 4 & $14.7 \pm 12.5$ & $28.3,3.0$ & $2.6 \pm 0.1$ & $2.7,2.5$ \\
\hline & $M$ & 2 & $4.4 \pm 1.3$ & $5.3,3.5$ & $3.2 \pm 0.9$ & $3.8,2.6$ \\
\hline
\end{tabular}

Sex-specific samples consisting of $n=1$ individual are not mean values. *mean, standard deviation, maximum, and minimum values were calculated with one less animal than the total $\mathrm{n}$ listed. $\mathrm{SD}=$ standard deviation, N/A= not applicable. 


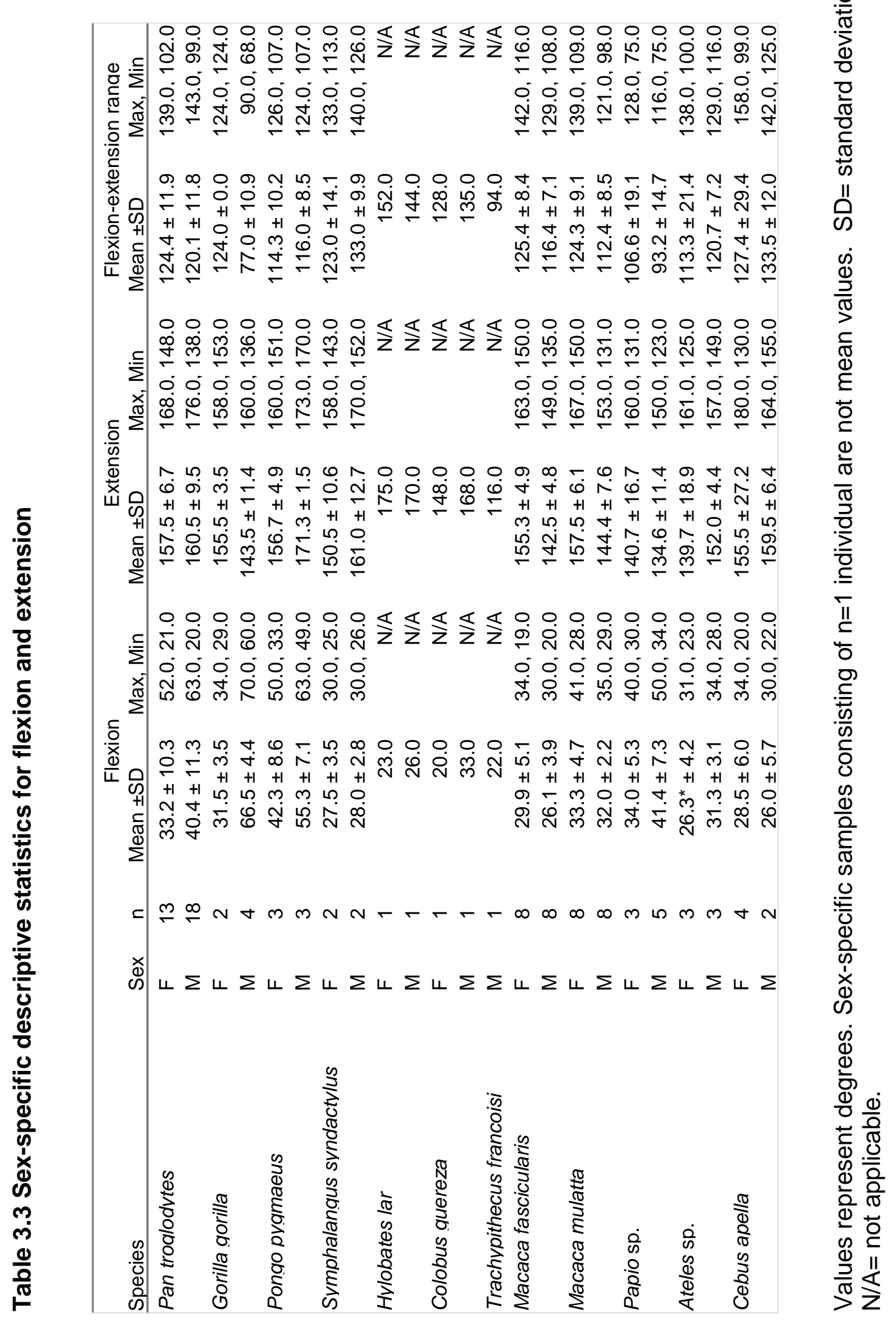




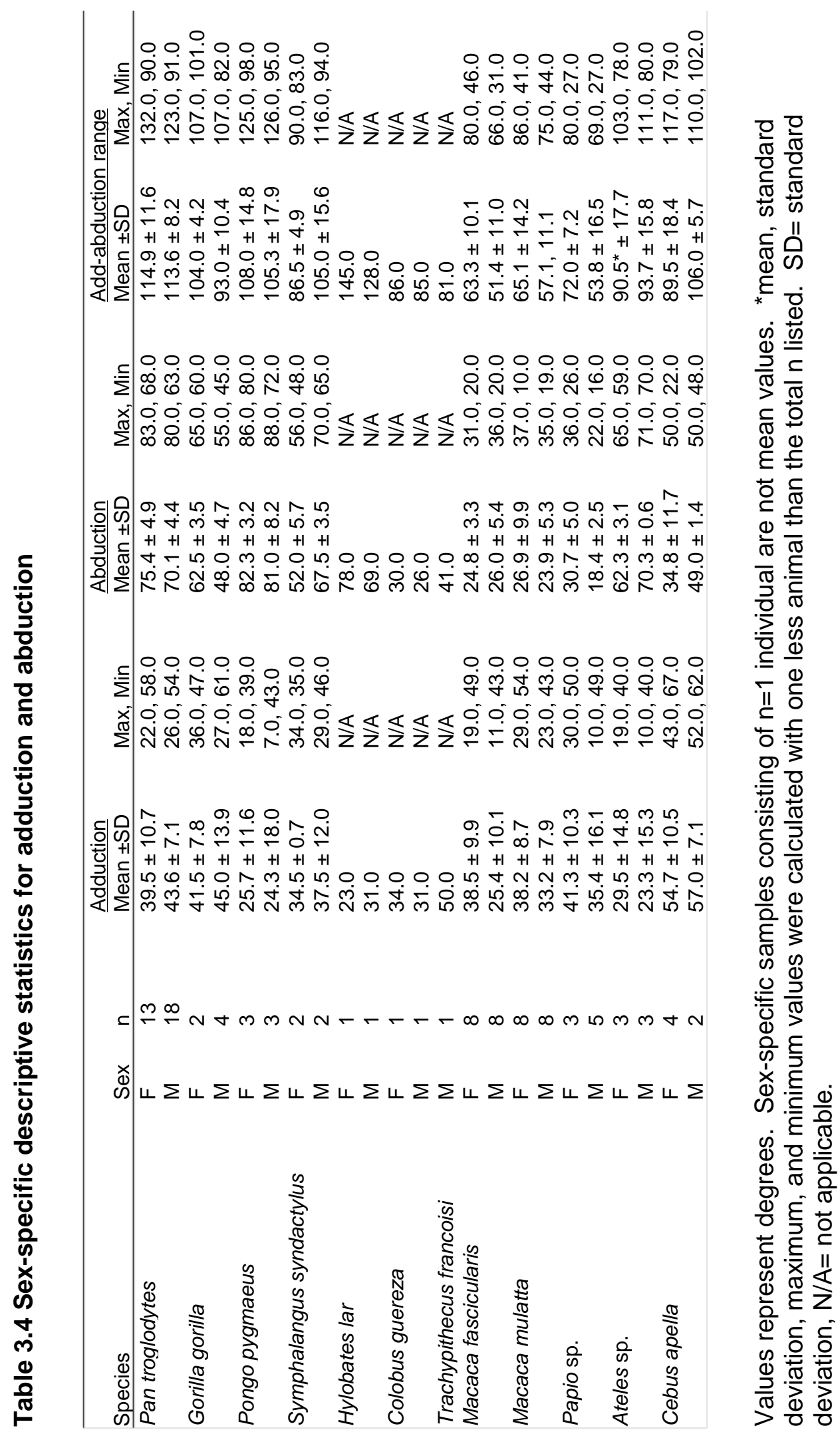

91 


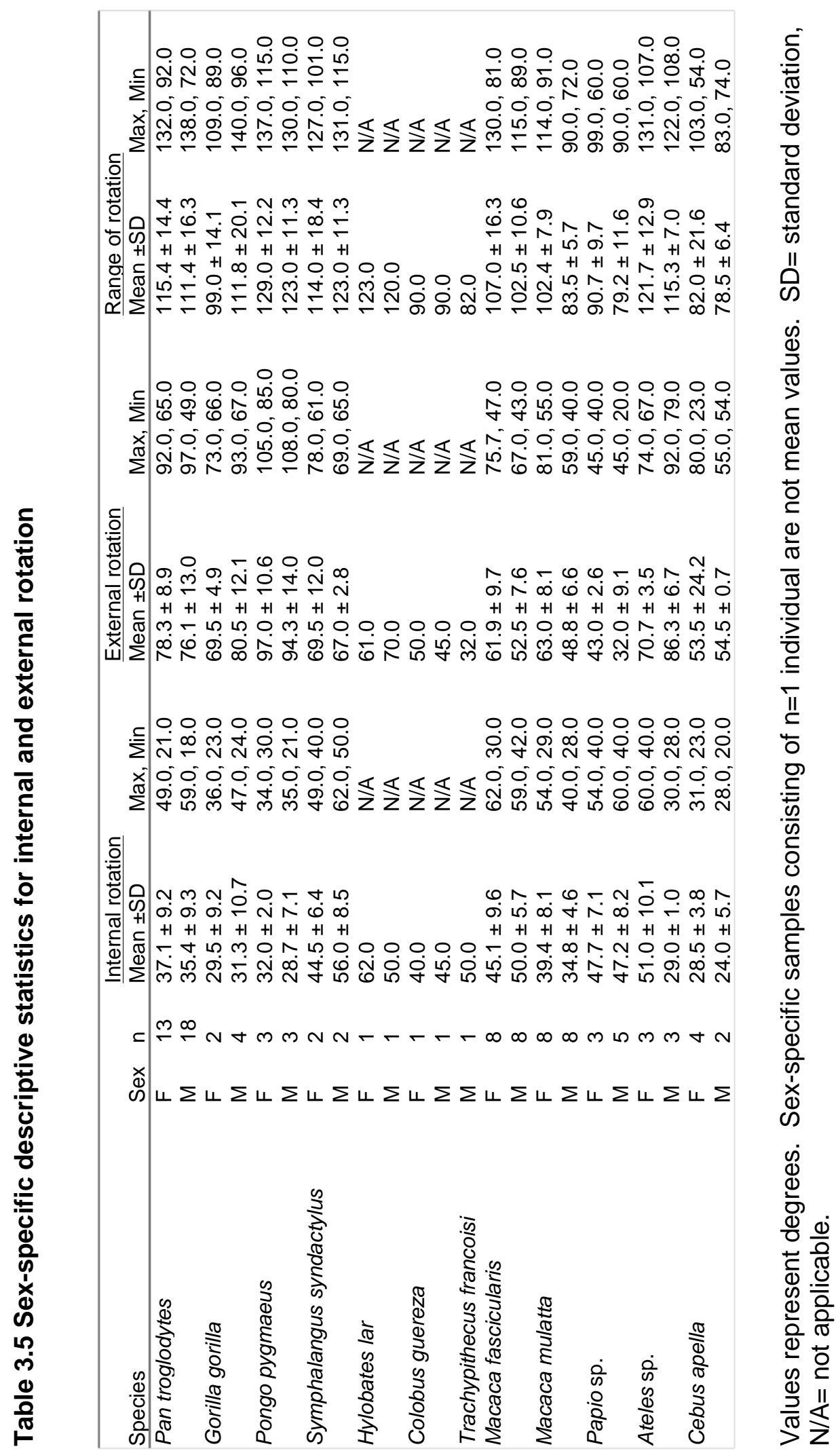




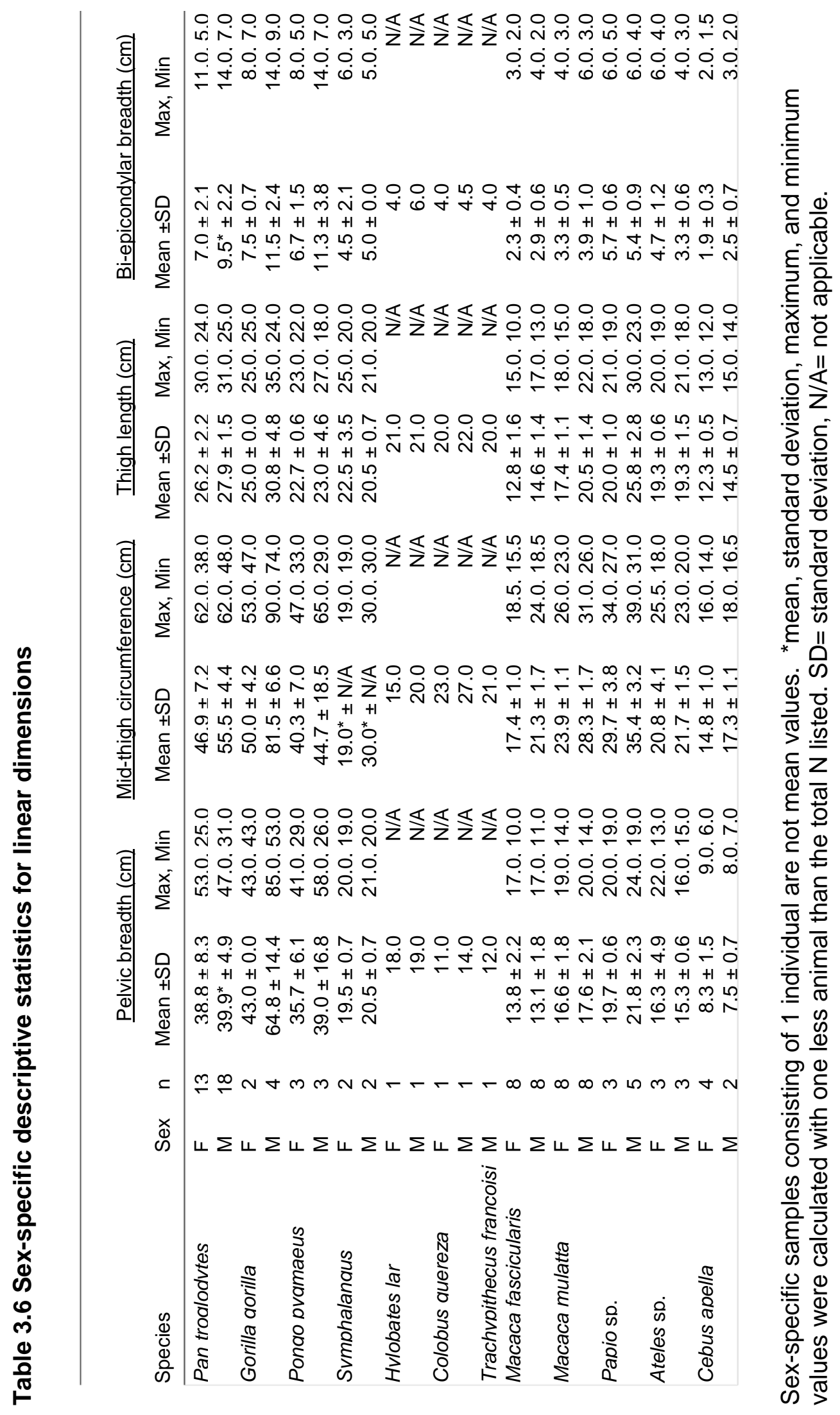




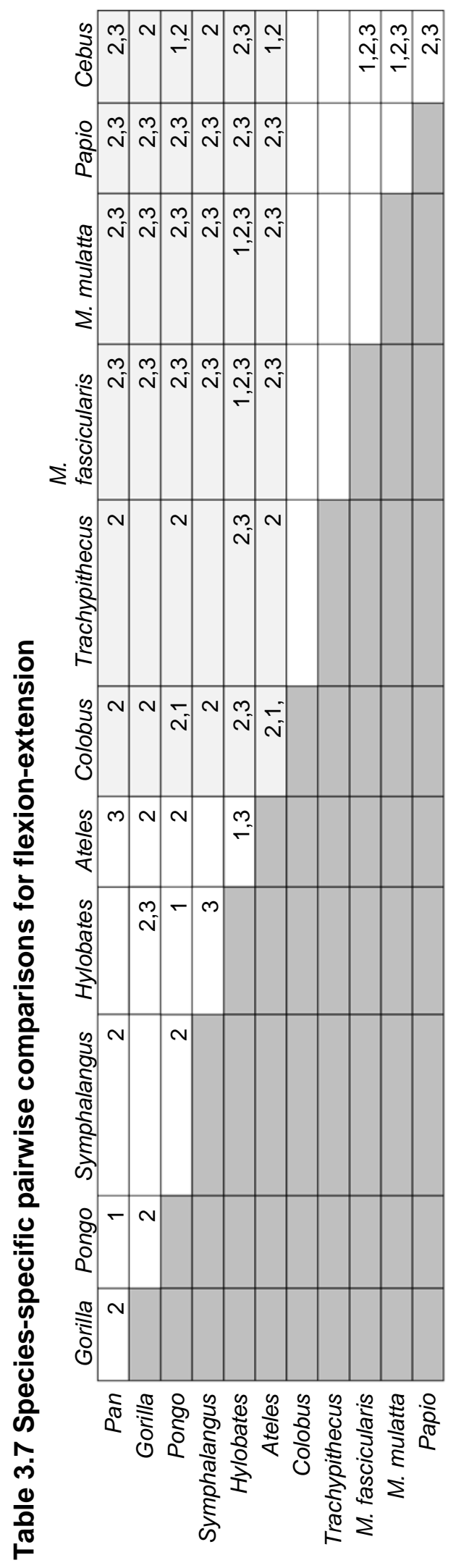

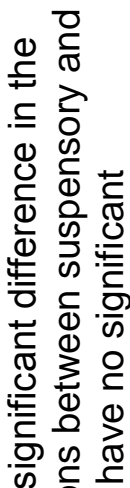

तర 애

임

Ф ․ㅡ

¿ 응

인 눙뭉

Ф

है 흠

है ญ

등 $\frac{\omega}{\overline{0}} \cdot \frac{0}{\pi}$

过

Ш खे 웡

훙 约음

응 능

的可 d

(). 은

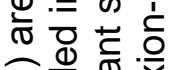

눙웡 중

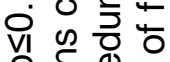

잉 ฏ

o

ธุ ส

을 을

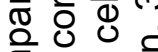

잉ㅎㅀ 흔

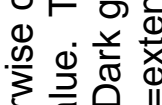

高

을 홍

I

d음 का

吉总

능

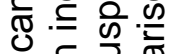

은 은 을

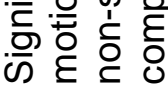




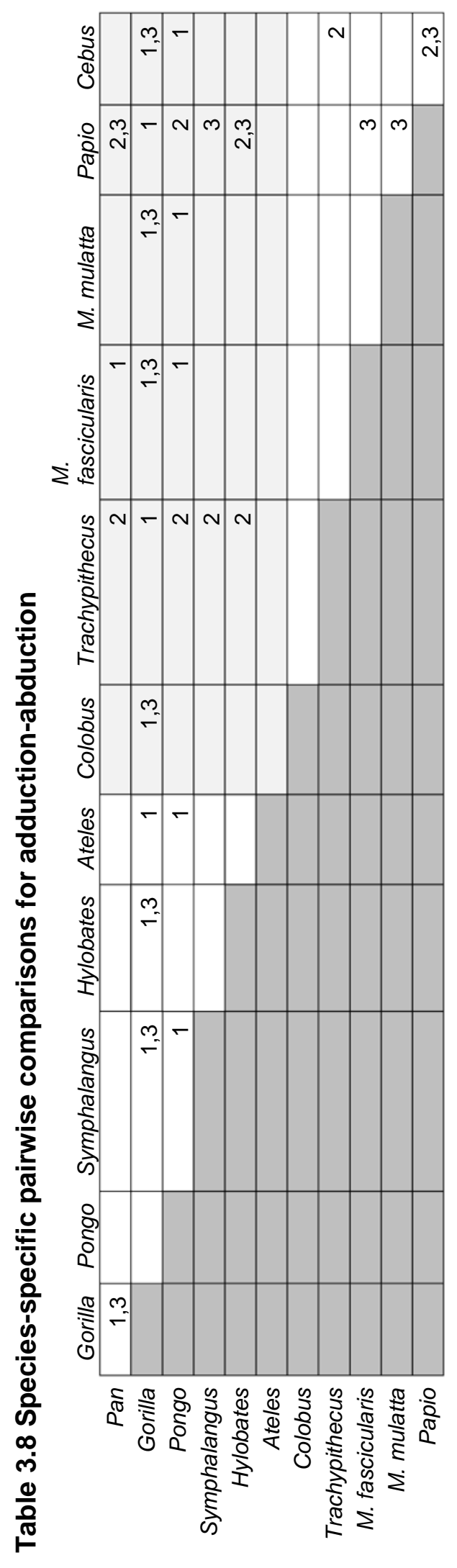

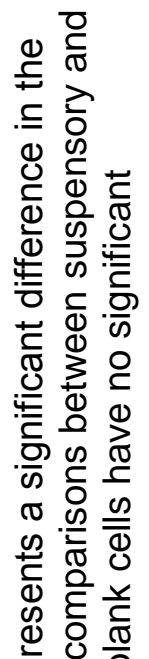

은 등응 음

ஸे

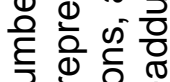

ऽ ब

등 응 응 응

Ш

ட் 느

ว드응

क ․ㅡ응

ญ.

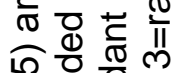

ㄷㅇㅇ응

ำ 을 응

응워원

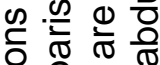

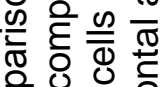

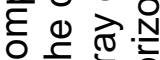

ठ등 흠

.

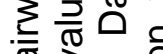

윰ำ 원

ด 든

증유

d음 की

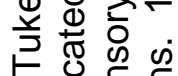

픙

तु $\subseteq \frac{2}{2}$ 은

는 은 옹

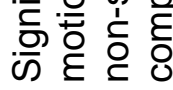




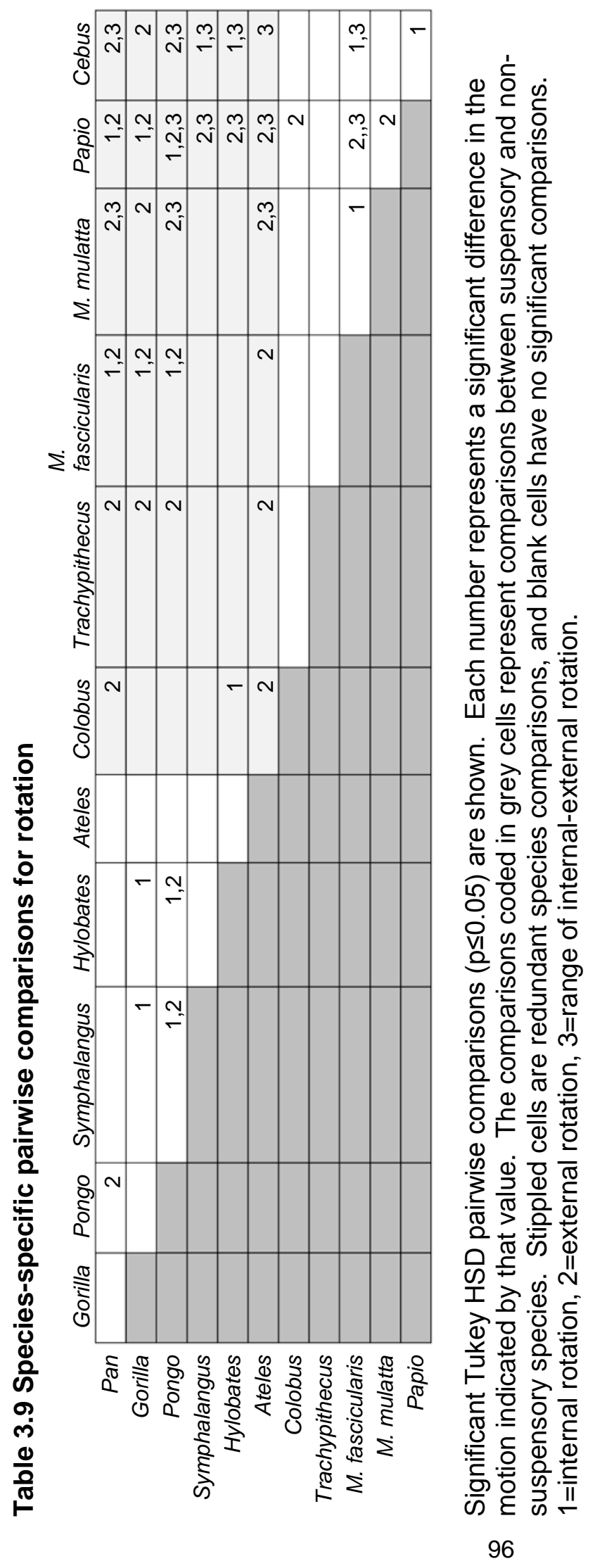




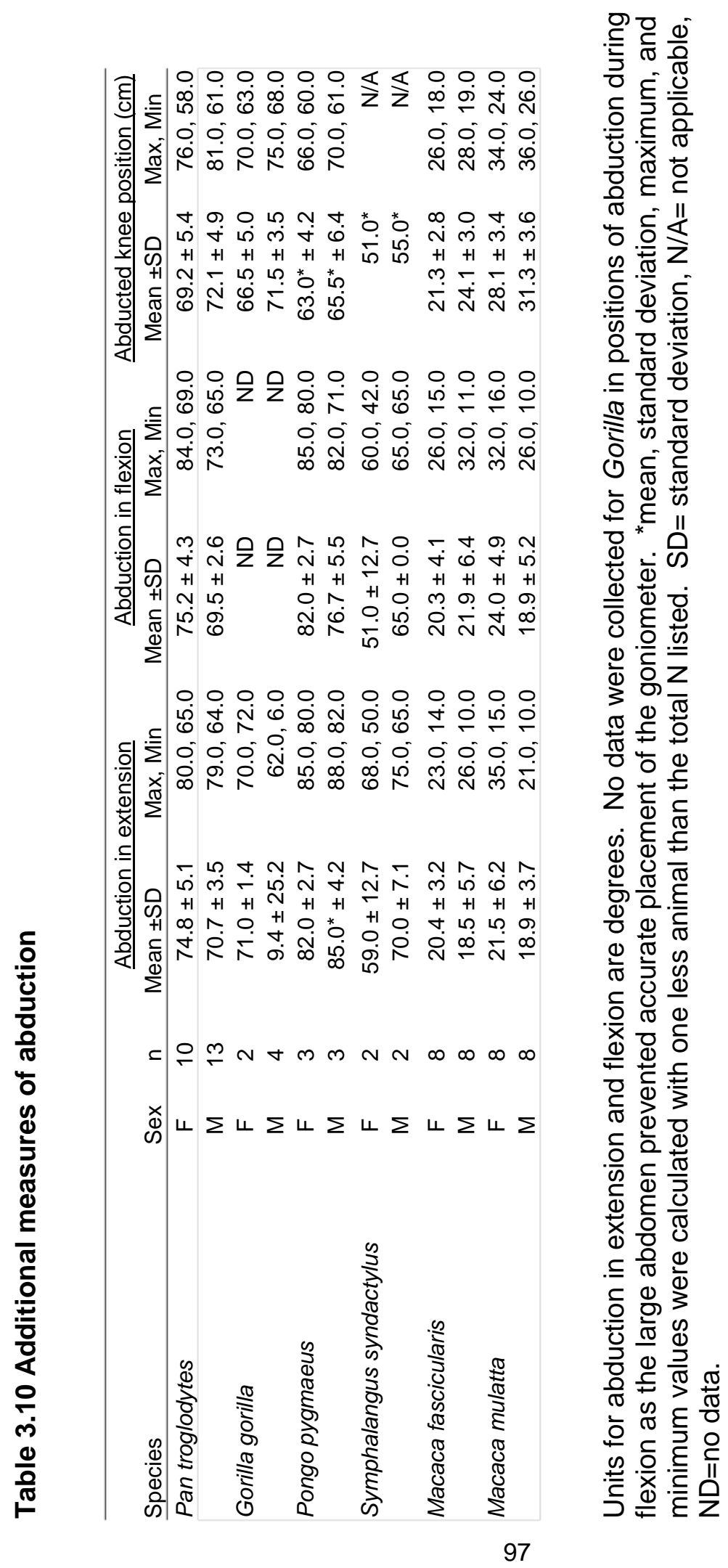


Table 3.11 Species-specific pairwise comparisons for additional measures

\begin{tabular}{|c|c|c|c|c|c|}
\hline & Gorilla & Pongo & Symphalangus & M.fascicularis & M.mulatta \\
\hline Pan & 1 & & 2,3 & $1,2,3$ & $1,2,3$ \\
\hline Gorilla & & 1 & 3 & $1,2,3$ & $1,2,3$ \\
\hline Pongo & & & $1,2,3$ & $1,2,3$ & $1,2,3$ \\
\hline Symphalangus & & & & $1,2,3$ & $1,2,3$ \\
\hline M.fascicularis & & & & & 3 \\
\hline
\end{tabular}

Significant Tukey HSD pairwise comparisons $(p \leq 0.05)$ are shown. Each number represents a significant difference in the motion indicated by that value. The comparisons coded in light grey cells represent comparisons between suspensory and non-suspensory species. Dark gray cells are redundant species comparisons, and blank cells have no significant comparisons. 1=range of abduction in flexion, 2=range of abduction in extension, 3=abducted knee position. 


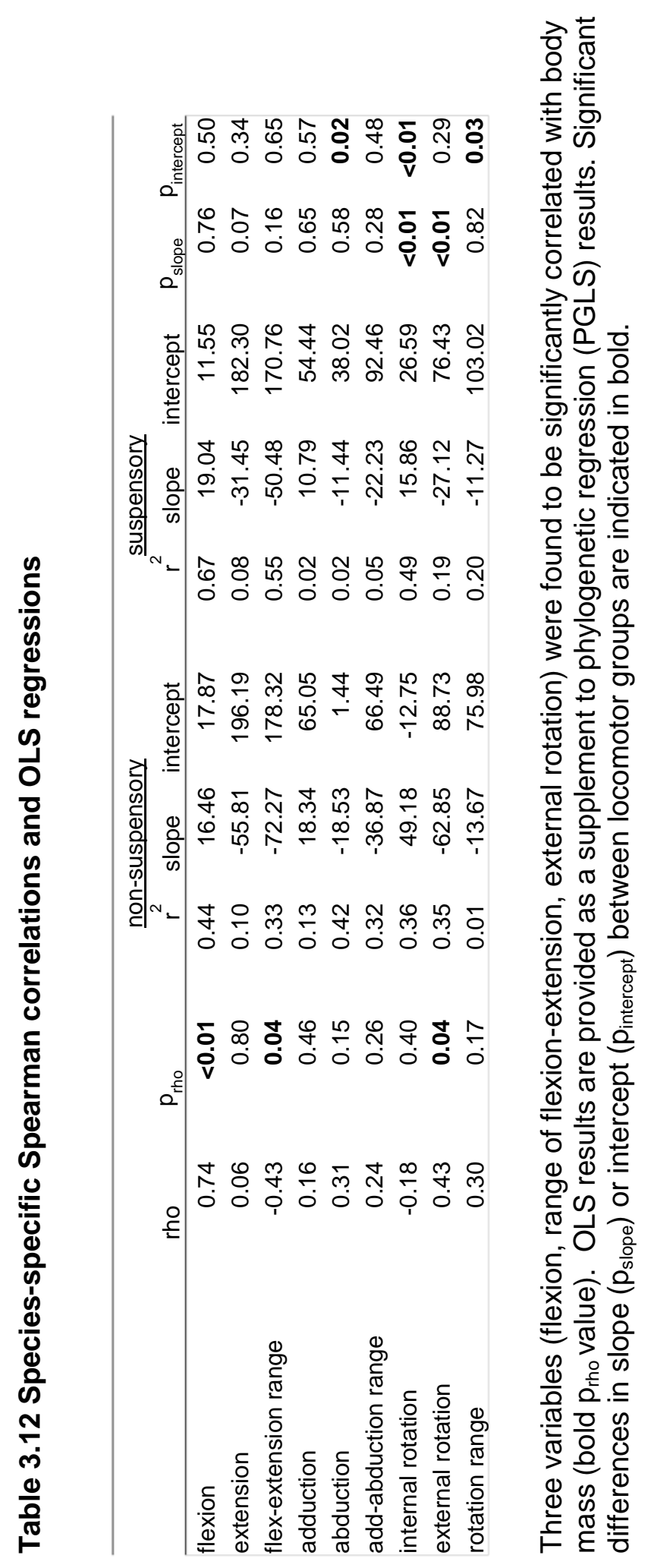




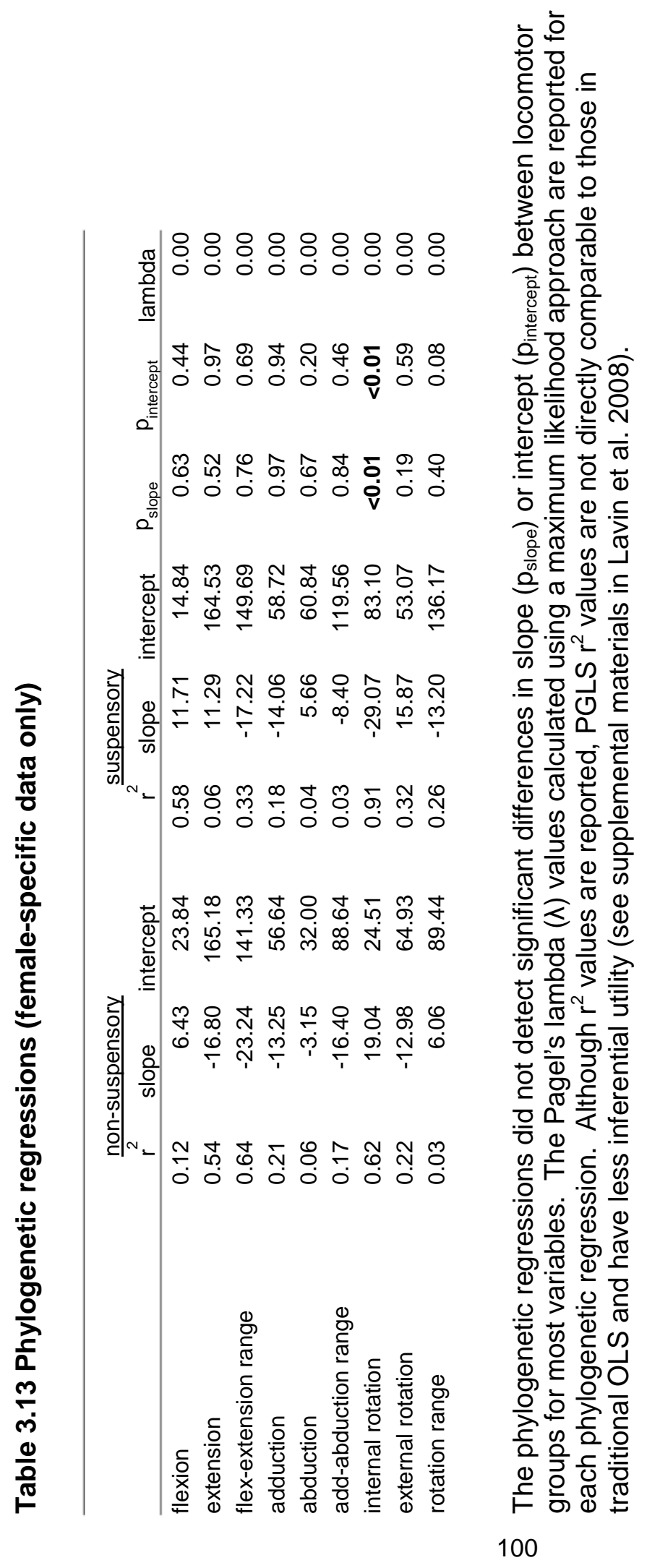



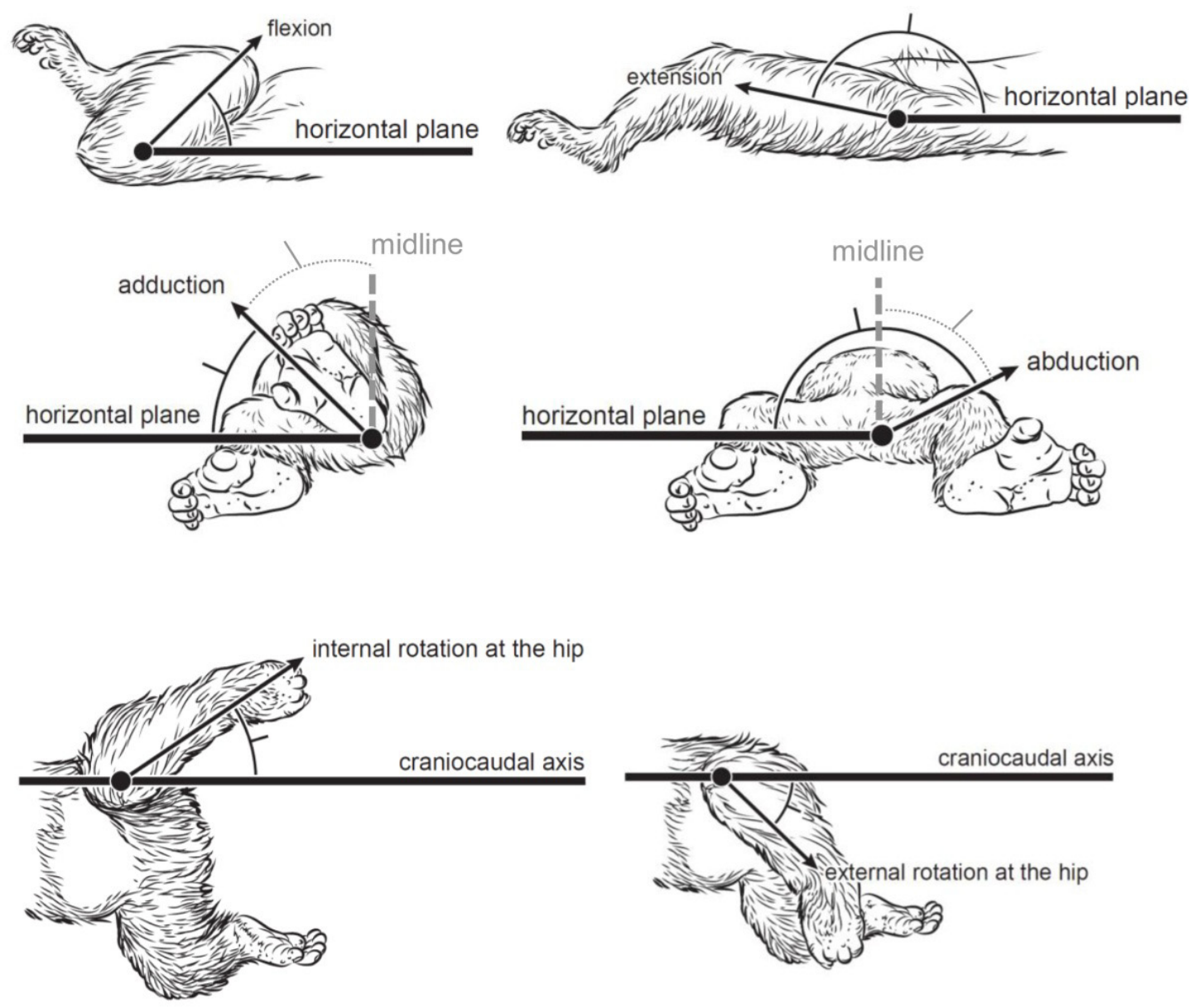

Figure 3.1 Measures

Adduction, abduction, internal rotation and external rotation were measured from the "horizontal" posture, which was defined as when the femur was $90^{\circ}$ (perpendicular) to the examination table. Adduction and abduction were measured relative to the horizontal surface of the examination table for accuracy but the angles were then adjusted to the midline (indicated in grey). Although best illustrated from a caudal view (shown above), adduction and abduction were measured cranially between the abdomen and thigh. 

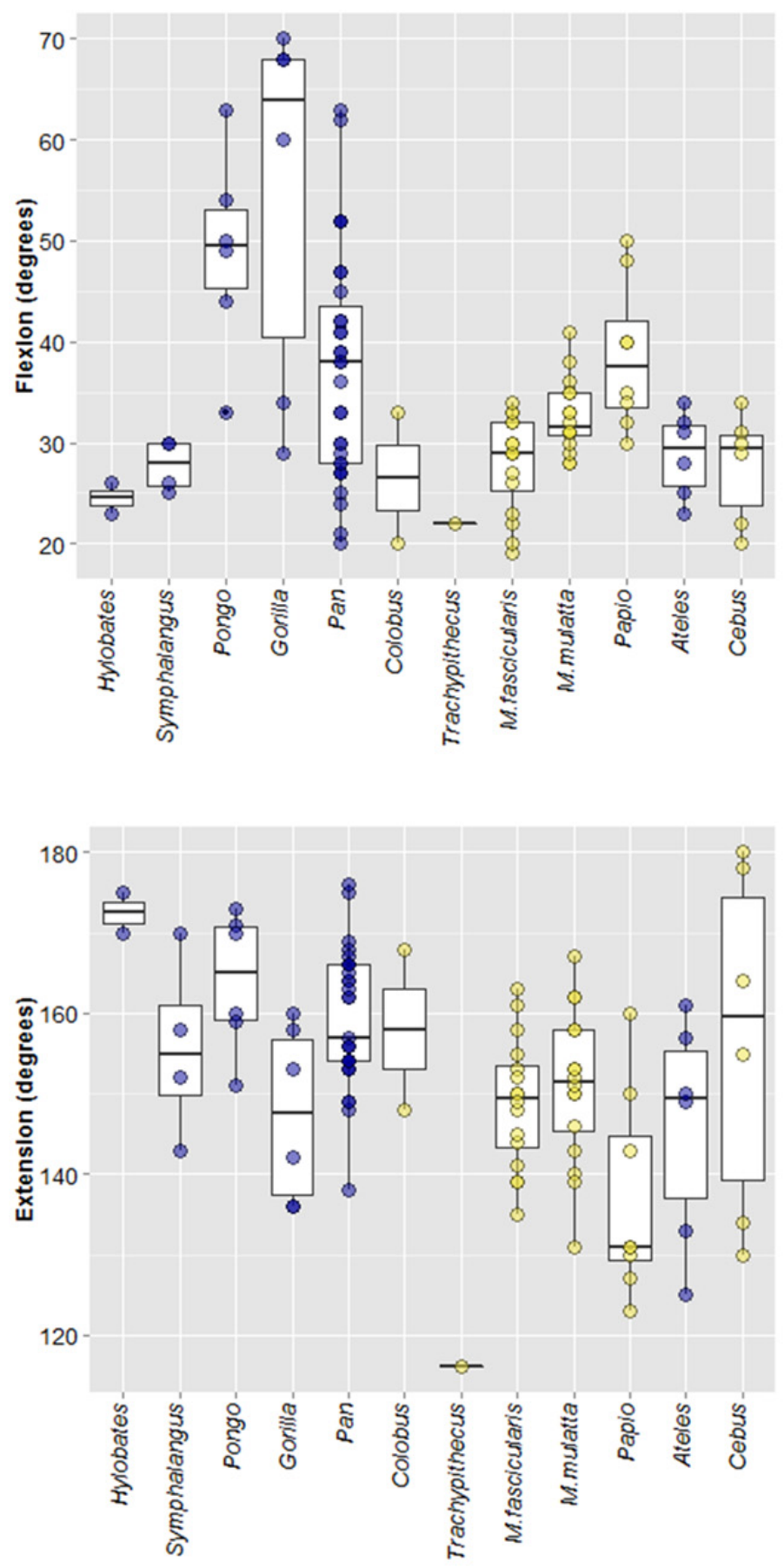

Figure 3.2 Cleveland dot plots of extension and flexion

Mean and interquartile ranges are indicated by the boxplots. Angles are significantly different between locomotor groups $(p<0.05)$. Note that smaller values for flexion reflect a higher range of motion (see Figure 3.1). Dark dots indicate suspensory data points and light dots indicate non-suspensory data points. 

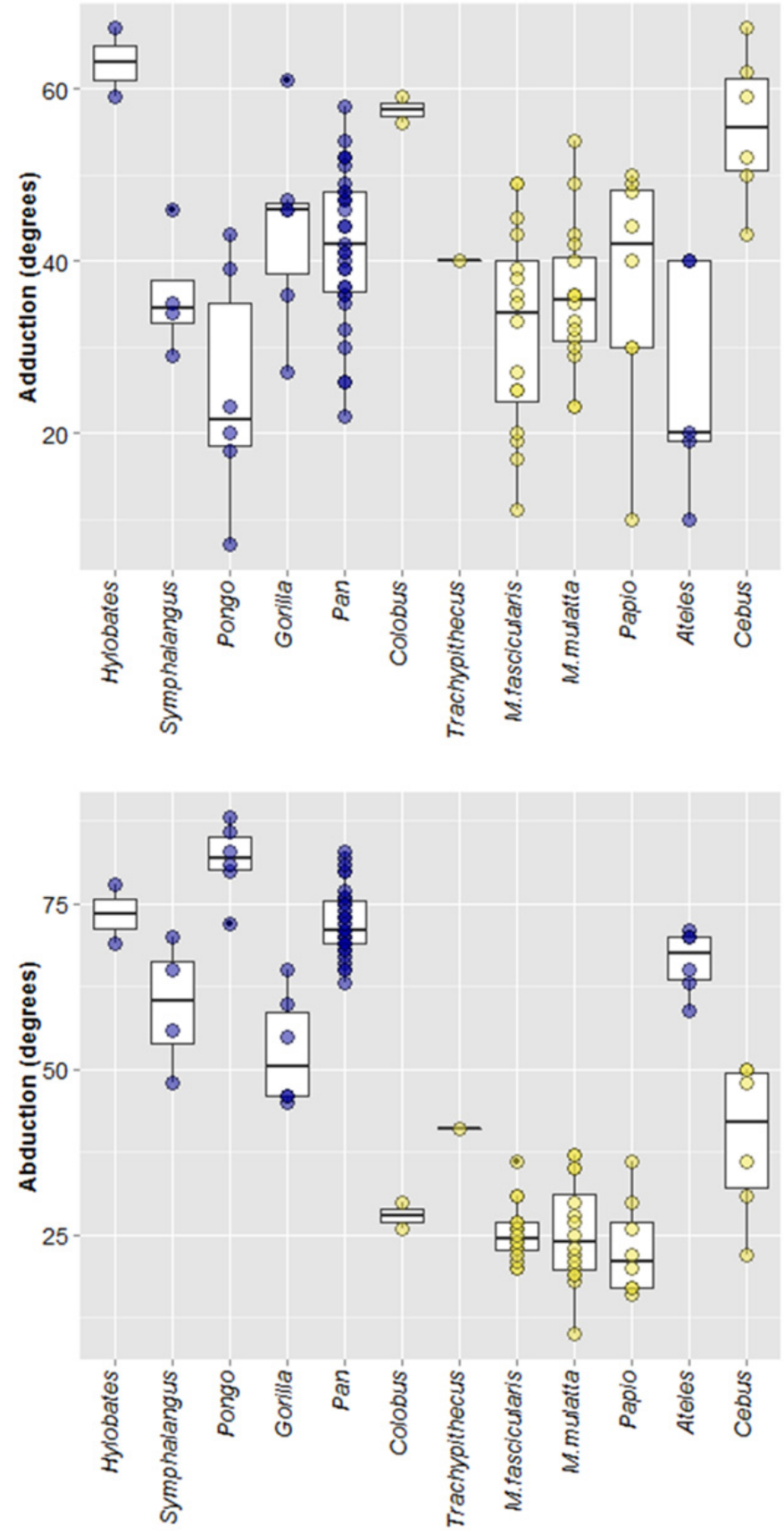

Figure 3.3 Cleveland dot plots for abduction-adduction

Mean and interquartile ranges are indicated by the boxplots. Abduction, but not adduction, is significantly different between locomotor groups $(p<0.05)$. Dark dots indicate suspensory data points and light dots indicate non-suspensory data points. 

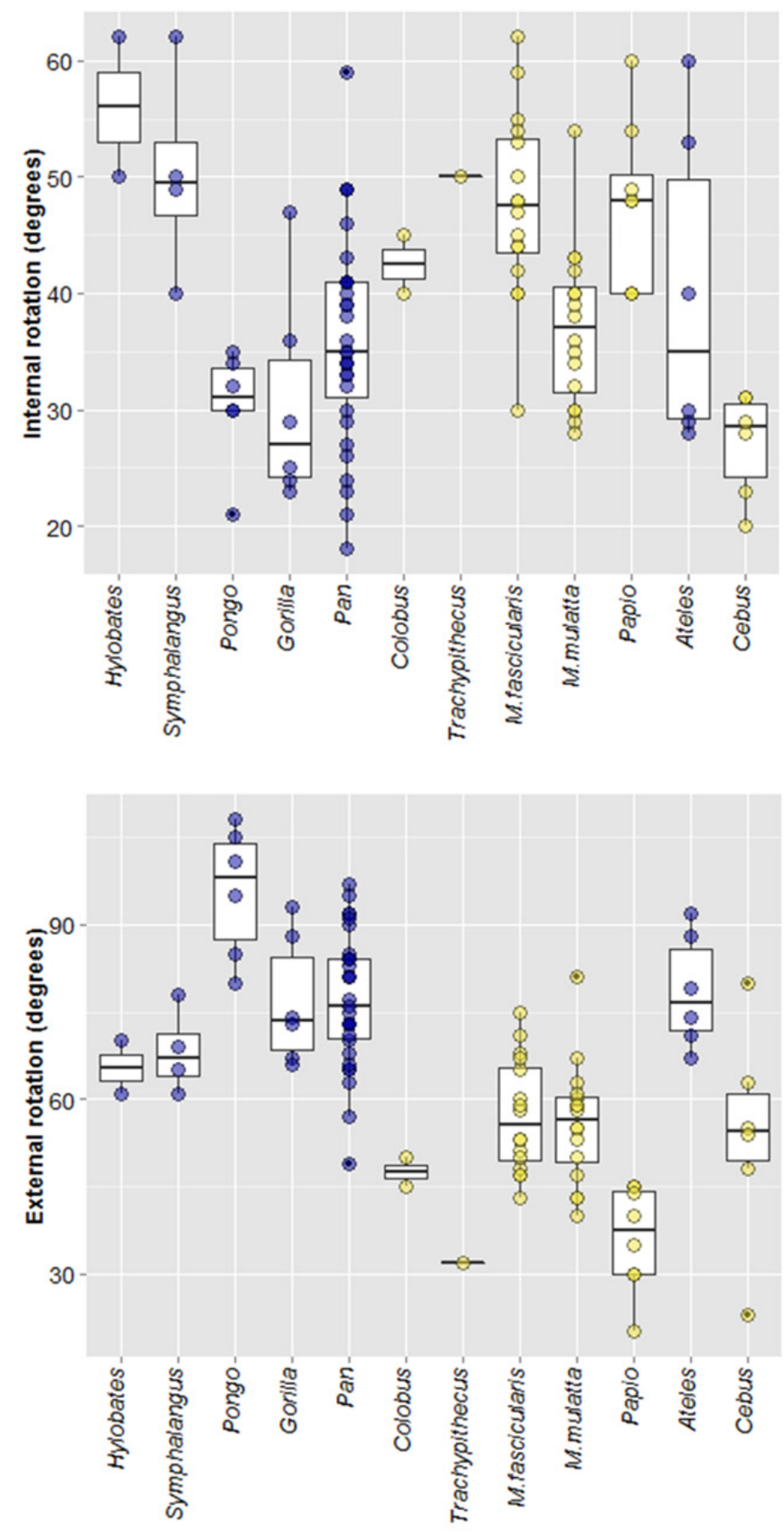

Figure 3.4 Cleveland dot plots for internal-external rotation

Mean and interquartile ranges are indicated by the boxplots. Angles are significantly different between locomotor groups $(p<0.05)$. Dark dots indicate suspensory data points and light dots indicate non-suspensory data points. 

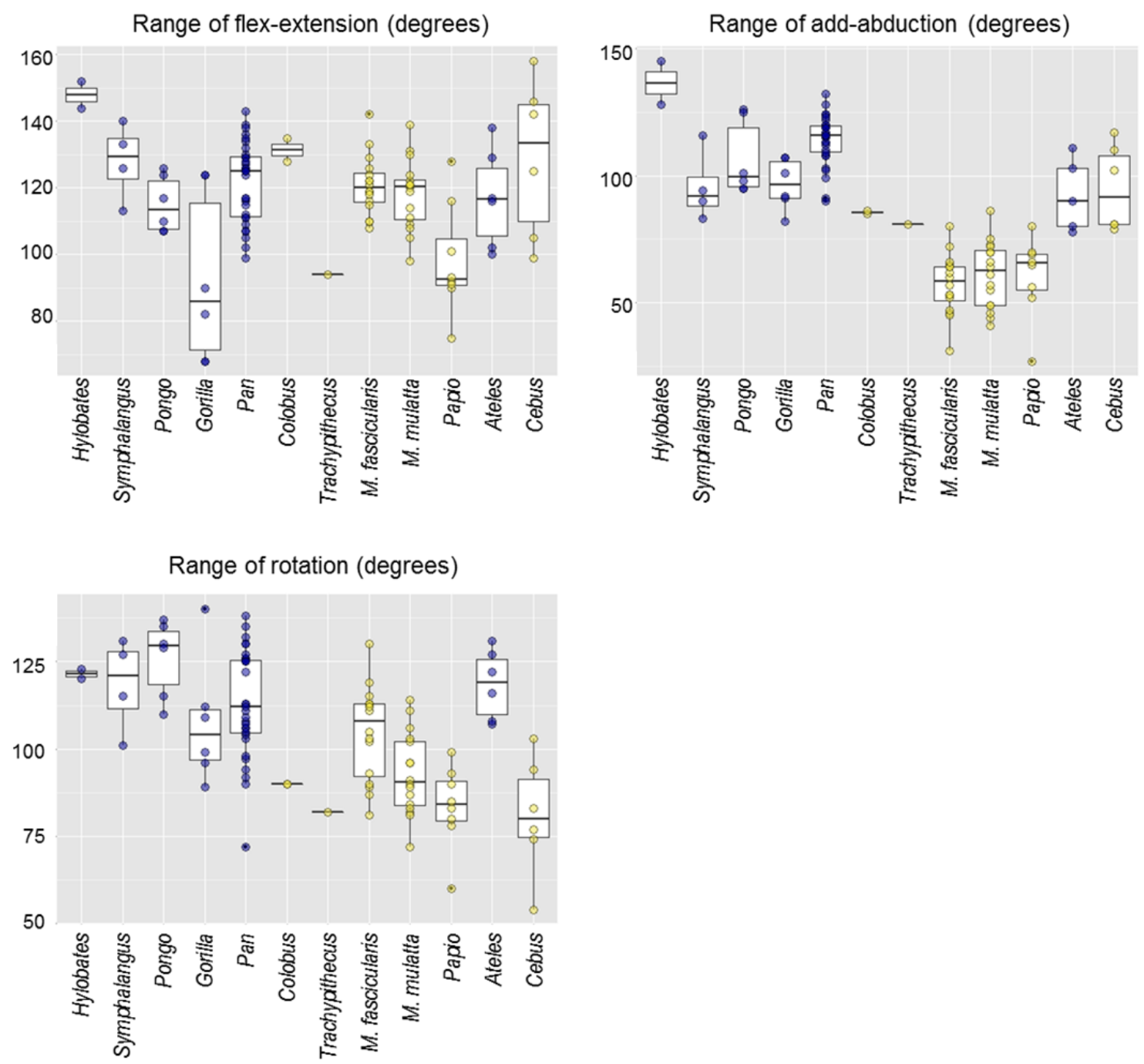

Figure 3.5 Cleveland dot plots for ranges of flexion-extension, adductionabduction, and internal-external rotation

Mean and interquartile ranges are indicated by the boxplots. Dark dots indicate suspensory data points and light dots indicate non-suspensory data points. 

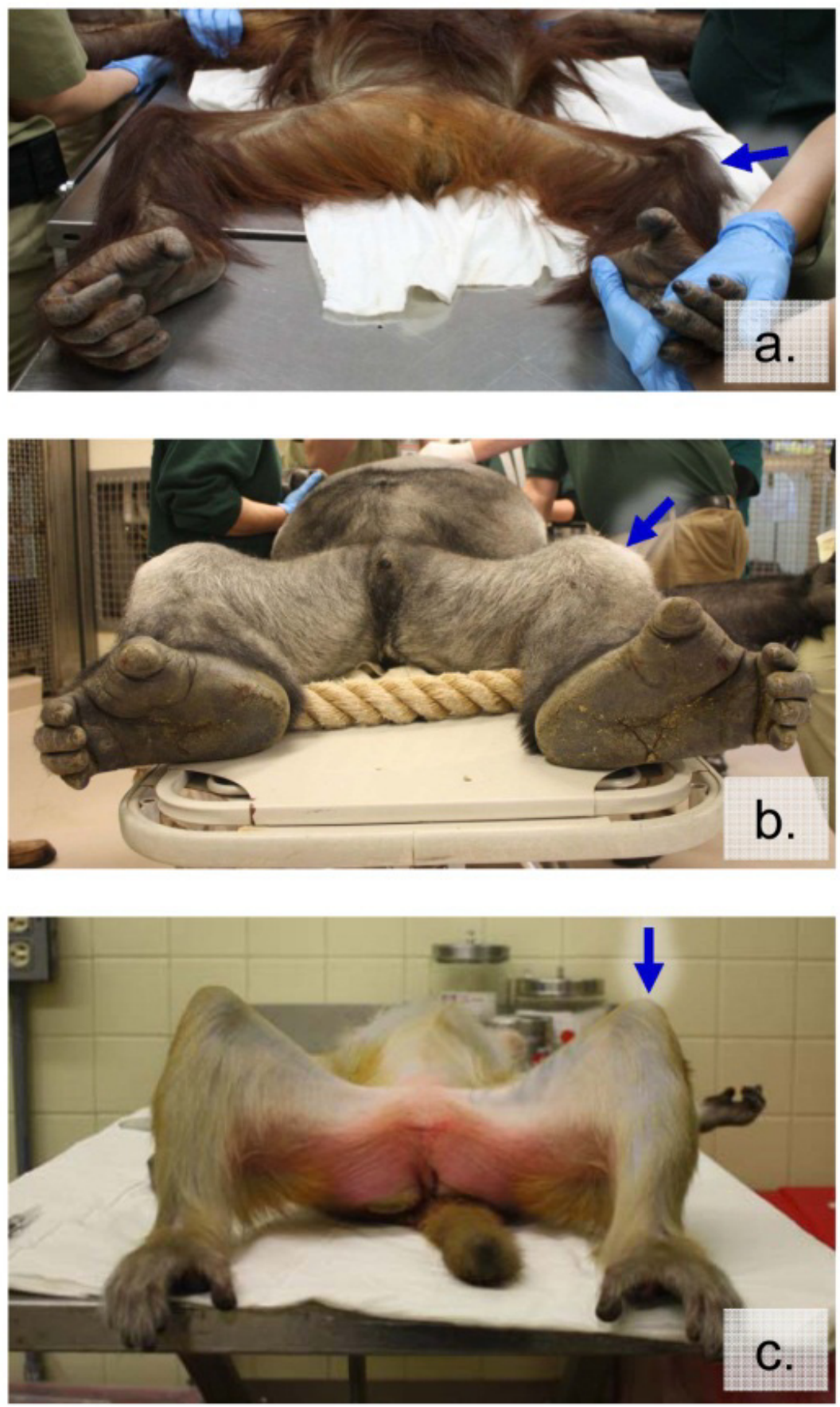

Figure 3.6 Abduction ability tied to lateral rotation

Examples of passive positions of abduction for (a) Pongo pygmaeus, (b) Gorilla gorilla, and (c) Macaca mulatta. The patella is indicated by blue arrows to highlight the external rotation that accompanies abduction in suspensory species. Note the lateral knee orientation (externally rotated thigh) in Pongo and Gorilla and the anterior orientation (non-rotated thigh) in Macaca. 


\section{CHAPTER 4: IN SILICO MODELS OF HIP JOINT ABDUCTION ABILITY}

Abduction was found to discriminate suspensory and non-suspensory taxa more than any other movement at the hip in a sample of live animals (Chapter 3 ). This finding, using empirical data, provides a basis for reconstructing anthropoid range of femoral abduction.

A model for quantifying hip abduction was developed following work of MacLatchy (1995, 1996, 1998), MacLatchy and Bossert (1996), Jenkins and Camazine (1977), and Kapandji (1970). Simulations of hip abduction were performed here on a large sample of anthropoids, following the principles outlined in these previous works for how a joint should be positioned during normal loading. The novel modeling approach integrates laser scans, microscribe landmark data, and shape analysis software to digitally articulate the hip joint. Anthropoid range of femoral abduction and the functional outcome of hip abduction, the abducted knee position, were reconstructed using 3D polygonal models of primate pelves and femora.

The in silico models were validated in a subset of taxa for which in vivo data were also available (Chapter 3), allowing quantification of how far models of joint movement deviate from empirical measurements collected on primates in vivo. This research design makes it possible to assess how closely digitally articulated hip joints reflect the limits imposed by surrounding soft tissues and provides a validated method of inferring functional abilities when only the bones are present (as in fossil hominoids). 


\section{OBJECTIVES}

First, extant suspensory anthropoids were tested for significantly increased ranges of abduction in silico compared to non-suspensory anthropoids. The ability to discriminate between locomotor groups by measures of abduction was tested by means of discriminant function analysis, and allometric scaling of hip joint abduction was assessed by standard major axis regression. Second, the hypothesis that there is a predictable relationship between hip abduction modeled in silico and that measured in vivo is tested. Analysis of variance (ANOVA) and pairwise multiple comparisons measure the relationship between data types at the locomotor and species levels.

\section{SAMPLE}

The in silico sample described here was used in analyses in both Chapter 4 and Chapter 5. Microscribe landmark data and laser scan data were collected for the pelves and femora of 252 extant anthropoid primates (Table 4.1). All individuals were judged to be adults based on femoral epiphyseal fusion. All individuals were wild shot specimens with the exception of 22 individuals (see specimens listed in Appendix B), which were included to supplement the sample sizes for high priority taxa for which wild specimens were unavailable. This total sample used in this project is comparable in size and diversity to other laser scan projects (Tocheri et al. 2005; Marzke et al. 2010). An ideal sample would include 20 sex-matched individuals per species but sample sizes are constrained by the known postcranial skeletal samples available in domestic and international museums. However, the sample included an independent test of the morphology-behavior association proposed for hominoids by including Ateles-Cebus. All taxa in the sample have locomotor behavioral data (see Chapter 2). 
The laser scan and landmark coordinate data used in this study were collected by multiple researchers (discussion of intra- and inter-observer error below). The author collected most of the data from specimens at the United States National Museum (USNM), Museum of Comparative Zoology at Harvard (MCZ), Cleveland Museum of Natural History $(\mathrm{CMNH})$, American Museum of Natural History (AMNH), Naturalis Leiden (ZMA), Bavarian State Zoological Collections (ZSM), and the National Museums of Kenya (KNM). To increase the sample size, laser scan data collected by Dr. J. Michael Plavcan (Department of Anthropology, University of Arkansas) were included in the sample. In addition, some microscribe landmark data collected by Dr. Carol Ward (Pathology and Anatomical Sciences, University of Missouri) were used (data from Ward 2009). The data collected by Plavcan and Ward were collected at locations listed above, as well as at the Royal Museum for Central Africa (MRAC), University of Zurich (UZIA), Powell Cotton Museum (PCM), and Field Museum of Natural History (FMNH).

\section{LASER SCAN METHODS}

\section{Scanning and Polygonal Model Rendering}

The left hipbone, or os coxa, and femur were scanned for each specimen. Right hipbones and femora were substituted when the left was damaged, as there was no systematic asymmetry in hipbone shape when the original specimens were examined. Scan data collected by the author and Carol Ward were collected with a Next Engine Desktop 3D Scanner (NextEngine, Malibu, CA, USA) and scan data collected by J. Michael Plavcan were collected with a Konica-Minolta Vivid 9i (Konica-Minolta, Tokyo, Japan). Laser scan data collected using different scanners, scan protocols, and even data point density are equivalent in volume and surface area (Guidi et al. 2008; Aguilar et al. 2009; Sholts et al. 2010; Slizewski et al. 2010). The low level of difference 
observed between Konica-Minolta and Next Engine scanners is comparable to differences achieved within scanning trials of the same scanner (Sholts et al. 2011).

Multiple NextEngine scan protocols were necessary for this project because NextEngine scanners have optimal settings for objects of different sizes, opacities, colors, and densities. The scan protocol was determined on a case by case basis, with priority given to scan mode determined by object size. Large bones $(>12 \mathrm{~cm}$ in any dimension), which comprised the majority of the sample, were scanned in the Wide Mode. Clean, non-greasy bones were usually scanned in a single $360^{\circ}$ iteration followed by single-shots of the ends of the bone. Greasy specimens, such as many platyrrhine skeletons, required additional $360^{\circ}$ scans to compensate for the laser diffraction that occurs as a result of the grease. In some cases, it was necessary to scan very large bones (>25 cm, such as Gorilla pelves) in Extended Mode. Small bones $(<12$ $\mathrm{cm}$ in all dimensions, such as Cebus pelves) were scanned in the Macro Mode. However, these small bones were often partially transparent due to their thinness, requiring multiple $360^{\circ}$ iterations to capture the object and possibly talc coating to increase opacity of the specimen (if permitted by curatorial staff). Prior to scanning, all pelves were oriented on the NextEngine turntable in a safe, steady position using casting putty and the assistance of the NextEngine part gripper. Unless it was considered unstable, the preferred position for $360^{\circ}$ iterations was with the iliac blade down for pelves and with the femoral head oriented up for femora. All scans were collected using high-definition settings (HD). Additional detail can be found on NextEngine scan setting options from the manufacturer (NextEngine 2012) and scan methodology publications (Tocheri 2009; Friess 2012). 
NextEngine laser scan point cloud data for each hipbone and femur were aligned in the Next Engine ScanStudio HD Pro proprietary software, and exported as a polygonal model (wavefront or .obj format). Scan processing was done during downtime at museums to ensure quality data were collected and to allow re-scanning of specimens, if necessary. Further scan processing, or "cleaning" to remove irregular topographies produced during scanning, was completed using the IMEdit module of PolyWorks V 11.0 software (InnovMetric, Québec, Canada). As in other studies using NextEngine data (e.g., Tocheri et al. 2011; Garvin and Ruff 2012), the laser scan data were edited in an external software program due to the limited options for cleaning the polygonal model in Scan Studio.

The following Konica-Minolta scan protocol has been provided by J. Michael Plavcan. Most specimens were scanned with the telephoto lens, although larger specimens were scanned with the medium lens. Most specimens were positioned between $600-900 \mathrm{~mm}$ from the lens and scanned in two overlapping halves, which could then be aligned. Konica-Minolta point cloud data collected by Plavcan were first manually assembled using the Konica-Minolta Polygon Editing Tool software. Alignments were then fine-tuned to produce maximum convergence and 1:1 point sampling between scans using the IMAlign module of PolyWorks. Overlap was reduced and point clouds merged with medium smoothing to produce a 3D polygonal model using the IMMerge module. The polygonal models of each pelvis were imported into the IMEdit module of PolyWorks for final processing and cleaning. 


\section{MICROSCRIBE LANDMARK METHODS}

\section{$\underline{\text { Pelvis articulation methods and landmarks }}$}

Microscribe landmark data eliminated the need to laser scan both halves of the pelvis and sacrum by providing 3D landmarks that can be used to align the hipbone in the PolyWorks virtual environment. This step substantially reduced the time and costs of this project, and accuracy can be quantified in this method of digital articulation, unlike most other methods of digital articulation.

First, the left and right hipbones and sacrum were articulated using putty and rubberbands. The thickness of the putty at the pubic symphysis and sacroiliac joints is approximately $3 \mathrm{~mm}$, following Li (2002) and Ward (2009). The 14 landmarks used in this analysis (Table 4.2) were indicated on the pelvis by a single pencil point. The microscribe data supplies 5 landmark coordinates of the left os coxa (ASIS, PIIS, dorsal acetabulum, center of acetabular fossa, and ischial spine) and 9 points used to establish the lumbosacral, midline, and sacral planes (Figure 4.1). These landmarks were selected because they provide information about anatomical planes and because they are easily observed on most pelves. The pelvis was then propped up by foam blocks and/or putty so that the landmarks were fully accessible from the ventral surface. Microscribe landmark data were then collected on the articulated pelvis using an Immersion (San Jose, California) MicroScribe 2GX. After completion of microscribe data collection, landmarks were removed using a white eraser.

\section{$\underline{\text { Pelvis landmarking error study }}$}

The author conducted a repeatability study of 10 landmarking trials to calculate (intraobserver) precision in measuring 3D landmark location. A chimpanzee pelvis 
housed in the Ward Lab (University of Missouri) was articulated following methods described above. The landmarks (Table 4.2) used in this study were digitized 10 times on August 2, 2011. Following the methods described in Singleton (2002), the distance of each landmark from the landmark centroid was calculated for each trial. The average distance for all trials for each landmark was also calculated. Mean deviations for each landmarking trial were calculated and the root mean square error (RMSE) was calculated for each landmark (Table 4.3). Singleton (2002) uses the quadratic mean, or root mean square error, to represent the intraobserver error. The author's mean RMSE is 0.89 , which is considered acceptable given the large size of the primate pelves. The author's microscribe precision averaged $0.35 \mathrm{~mm}$ across landmarks, and did not exceed $1.67 \mathrm{~mm}$ deviation from the centroids, a level of precision similar to other landmarkbased studies (Singleton 2002; Frost et al. 2003). The values reported in Table 4.3 suggest that the author has a very high level of repeatability in selection of landmarks ${ }^{8}$.

\section{$\underline{\text { Pelvis articulation error study }}$}

The author conducted a similar repeatability study to calculate (intraobserver) precision in articulating pelves. The biggest source of error in articulating pelves is almost certainly variation in the putty thickness, which is necessary to hold the two hipbones and sacrum together. The previously described chimpanzee pelvis housed in the Ward Lab was articulated 5 times during a 12 hour period on August 2, 2011. After each articulation session, the 14 landmarks used in this analysis were digitized and recorded. Because it was necessary to move the pelvis between articulations, the percent difference between 4 interlandmark distances were examined (Table 4.4). The

\footnotetext{
${ }^{8}$ Some landmark data used in this study were provided by Ward (2009), who has also verified her high levels of precision in landmark selection in an independent error study (personal communication).
} 
distances were selected because they span both the sacrum and pelvis, which is necessary to gauge the precision with which the bones were aligned to each other. A comparison of the interlandmark distances (Table 4.5) found less than $1.2 \%$ difference between all maximum and minimum interlandmark distances in the 5 articulation trials. These values suggest a very high level of intraobserver repeatability in articulation technique.

\section{DIGITAL ALIGNMENT METHODS}

\section{$\underline{\text { Pelvis alignment to landmark coordinate data }}$}

For each specimen, the laser scanned os coxa was aligned to its matching microscribe landmark coordinates in the PolyWorks IMInspect module. First, the microscribe $x, y, z$ landmark coordinate data were saved as tabular data in text files (.txt) and imported into the IMInspect workspace. The PolyWorks virtual environment also works within an $x, y, z$ system and can recognize the numeric data as coordinates within its environment. The landmarks were manually identified as point objects, one of the PolyWorks geometrical primitives, giving the landmarks a three-dimensional anchor in the virtual environment. Midline and lumbosacral planes were then established using these point objects (Figure 4.2). A best-fit plane was established to point objects created from landmarks 6-10 (Table 4.2), corresponding to the pubic symphysis and sacral midline. A best-fit plane was also established from point objects created from landmarks $11-14$, collected on the cranial surface of the first sacral vertebra, and corresponding to the lumbosacral plane. A third plane was from landmarks 10, 13 and 14 , roughly corresponding to the ventral surface of the sacrum, to facilitate viewer orientation in images but is not used in this study. 
The hipbone polygonal model belonging to the landmarks was then imported into the IMInspect workspace. The model loads with 3D coordinates assigned by the scanner and so it must be aligned to the landmark coordinate data manually. Points were anchored on the pelvis model at the ASIS, PIIS, center of the acetabular fossa, ischial spine, and dorsal border of the acetabulum, in the same locations as microscribe landmarks 1-5 collected on the original specimen. The IMInspect center point alignment operation was then used to align the 5 points on the polygonal model to the points established from the landmark data, dragging the polygonal model into alignment along the midline plane in the process. It is worth noting that the midline landmarks collected on the pubic symphysis were collected with putty (approximately $3 \mathrm{~mm}$ thick) in between the two innominates, and so the midline plane should be just slightly medially off-set from the innominate.

\section{Femoral abduction constraints}

Maximum abduction was determined by digitally manipulating the femur within the acetabulum following a set of boundary conditions, which are largely based on biological and theoretical constraints outlined by MacLatchy (1995).

\section{Center of movement}

All movements of the femur were constrained around the hip joint center. For a ball and socket joint like the hip, the center of rotation of the hip joint is the center of the femoral head, which is the same as the center of the acetabulum (Pauwel 1980;

Brinckmann et al. 2002a). The hip joint center was calculated numerically using a digital sphere-fitting approach (described in "femoral abduction methods" below).

\section{Boundary constraints on mobility}


The fovea capitis was restricted to the confines of the acetabular fossa. Since the fovea is hypothesized to be confined to the acetabular fossa during normal joint loading (see extensive discussion in Chapter 2, as well as Kapandji 1970; Jenkins and Camazine 1977), the simulated movements of the femoral head within the acetabulum are limited by keeping the fovea capitis within the internal boundaries of the lunate surface (Figure 4.3). This constraint did not apply to Pongo, which does not typically have a subchondral ligamentum teres insertion.

In addition, the subchondral borders of the acetabular margin and femoral head were kept relatively congruent. Attention to the subchondral borders was the only subjective constraint but is an extremely important one because it was the primary method for assessing positions of the femoral head within the acetabulum in Pongo. This limit on movement can be influenced by joint geometry (personal observation), with more shallow or loose-packed joints being more difficult to estimate when the subchondral borders were not congruent. MacLatchy (1995) allowed "slight overlap" of non-articular surfaces with subchondral surfaces until one of the other constraints on movement had been met, although MacLatchy's study limited femoral movement before the acetabular margin would have impinged the femoral neck. In this study, this constraint was rarely met, as the maximum fovea position within the acetabular fossa was typically met before the subchondral borders overlapped.

A final boundary constraint on mobility was that bony elements cannot intersect other bony elements. Special attention was paid to the femoral neck and greater trochanter, which cannot intersect the bony pelvis during movements of the hip in vivo. This constraint was never met within this study or MacLatchy's $(1995 ; 1996 ; 1998)$ study. 


\section{Femoral abduction methods}

After aligning each pelvic polygonal model to its corresponding landmarks, the matching femur model was imported into the IMInspect workspace. IMInspect geometrical objects, or shape primitives, were necessary to establish certain landmarks for abducting the femur. All shape objects were established by a single observer (the author), reducing the potential for error. The following eight objects were created in the IMInspect workspace in order to align the femur within the acetabulum and to measure femoral abduction:

(1) A best-fit sphere was fit to the acetabular lunate surface by selecting the surface of the entire C-shaped subchondral lunate surface, making sure not to select surface points in the acetabular fossa or beyond the external acetabular borders, using the least squares sphere-fitting option. Data points that were more than 2.5 standard deviations from the average used to compute the sphere were rejected, and the sphere was recomputed without outlier points. This option was selected for all sphere-fitting procedures used in this thesis. Best-fit spheres following a pure least squares approach using PolyWorks operations can be fit with less than 1\% error within and between observers to both the acetabular lunate surface and the femoral head articular surface (Hammond et al. 2012; Plavcan et al. 2012; Hammond et al. 2013b).

(2) A point object was created at the numerical center of the acetabular sphere, representing the center of the hip joint.

(3) A best-fit sphere was fit to the femoral head by selecting the entire subchondral surface, making sure not to select surface points in the fovea capitis or beyond the external borders, using the least squares sphere-fitting option (Figure 4.5). 
(4) A point object was created at the numerical center of the femoral head sphere (Figure 4.5).

(5) A point object was anchored at the center of the fovea capitis, based on visual inspection. Because foveae are irregular and not necessarily deepest at their central point, it was not possible to use automated methods to identify the central point.

(6) A point object was anchored at the posterior intercondylar notch of the distal femur.

(7) A vector was fit to the femoral shaft by selecting the entire cylindrical diaphysis, beginning below the lesser trochanter and ending above initial flaring for the femoral condyles, using the least squares vector-fitting option (Figure 4.6). Data points that were more than 2.5 standard deviations from the average used to compute the vector were rejected, and the vector was recomputed without outlier points. Best-fit vectors can be fit with a high level of precision in PolyWorks, with a maximum difference $<2^{\circ}$ in orientation between vectors in an error study conducted for this thesis (Table 4.8).

(8) A plane was set perpendicular to the long axis of the ischium manually. It was not possible to identify a long axis using automated methods in species with very short ischia (e.g., Hylobates) and in most fossil specimens which have damage to the caudal ischium, and so it was necessary to use a manual technique for setting the plane through the ischium. From a lateral view, the long axis of the ischium was positioned left to right and then set an orthogonal plane through the ischium. The angle formed between the diaphyseal vector and the ischial plane was monitored using IMInspect measurement features.

After the creation of the shape primitives, the femur and pelvis contained all necessary components to begin abduction simulations. First, the best-fit spheres for the femoral head and lunate articular surfaces were made coincident via the IMInspect point 
alignment operation, positioning the femoral head within the acetabulum. The fovea point on the femoral head was then aligned to the point anchored to the center of the acetabular fossa to ensure the femoral head was positioned medially, and the point alignment operation was repeated for the femoral head and acetabular sphere center points. The femoral head was then rotated from the center of movement in the center of the hip joint to a position of maximum abduction where all other boundary constraints had been met.

During abduction simulations, the femoral shaft was kept in a neutral "horizontal" posture in an attempt to match the in vivo and in silico measures as closely as possible. The horizontal posture is intermediate in the flexion-extension range for quadrupeds ${ }^{9}$ (Figure 4.4), and was achieved by keeping the least squares best-fit vector of the diaphysis parallel to a transverse plane set through the ischium. More specifically, the horizontal posture is $90^{\circ}$ (varying by less than $+/-1^{\circ}$ ) to the long axis of the ischium (e.g., perpendicular). Thus, the horizontal posture is based on the position described in Chapter 3 but is measured relative to the long axis of the ischium rather than a horizontal examination Tabletop. The ischium was selected as the best approximation of the horizontal because it usually runs cranio-caudally and because it is present in multiple fossil primates. It is worth noting that there is variation in the dorsal flexion of the ischium, with colobine monkeys and hylobatids having a moderately flexed ischium and (non-human) hominids and atelines having straight ischia (Fleagle and Simons 1979; Fleagle and Anapol 1992; McCrossin and Benefit 1992). There could be some interspecific influence on the model from dorsal flexion of the ischium but it is not

\footnotetext{
${ }^{9}$ MacLatchy's (1995) study examined 2 femoral postures, highly extended $\left(0^{\circ}\right.$, or parallel, to the long axis of the ischium) and flexed (135 $5^{\circ}$ to the long axis of the ischium). According to MacLatchy (1996), however, she also examined a position $90^{\circ}$ and $45^{\circ}$ to the long axis of the ischium, finding the same results in both these positions.
} 
expected to be greater than that resulting from comparisons between in vivo and in silico data.

\section{Femoral abduction measures}

Maximum abduction was measured by (a) angular abduction of the femur relative to the midline plane and (b) by the physical distance between the knee and the midline plane (Figure 4.7). The angle of abduction was measured by calculating the angle formed between the diaphyseal vector (primitive \#7) and the midline plane. The physical distance spanned by the knee was measured as the shortest distance from the intercondylar notch point (primitive \#6) to the midline plane.

\section{QUANTITATIVE METHODS}

\section{Differences between suspensory and non-suspensory primates}

Angular abduction and knee position determined in silico were compared between suspensory and non-suspensory primates (full sample in Table 4.1) by locomotor group. Significant differences in abduction and abducted knee position were tested between suspensory and non-suspensory species by means of one-way analysis of variance (ANOVA). Boxplots were used to visualize differences between taxa. Gorilla beringei, Alouatta caraya, and Pygathrix nemaeus were plotted alongside extant taxa in figures but are not included in ANOVAs where suspensory and non-suspensory locomotor categories were used. Quantitative analyses were performed in R (version 3.0.1, R Core Team 2012). 


\section{Classification accuracy}

Linear discriminant function analyses (DFA) were used to find the accuracy in classifying in silico data by locomotor group (suspensory vs. non-suspensory). DFA is important for later analyses because it measures the strength of predictions for fossil group membership. First, the percentage of correct classifications by locomotor group for abduction (degrees) and abducted knee position $(\mathrm{mm})$ were calculated when sampling all individuals in both locomotor groups. Second, discriminant function analysis was then used to find the accuracy in classifying in silico data into a refined category (i.e., hylobatids, Gorilla gorilla, Pan, Pongo, Ateles, Cebus, cercopithecoids), in the case that this might provide additional specificity for fossil predictions. These refined categories were selected because they broadly correspond to taxonomic groups of more refined locomotor categories. That is, hylobatids are specialized brachiators, Gorilla gorilla and Pan frequently use terrestrial knuckle-walking, Pongo uses slow quadrumanous clambering, Ateles uses clambering and semi-brachiation, with Cebus and cercopithecoids using quadrupedalism. Gorilla beringei, Alouatta caraya, and Pygathrix nemaeus were excluded from DFA analyses, as in other analyses requiring extant species to be a priori assigned to a specific locomotor category. The DFAs included iterative jackknife reclassification techniques, and were performed in $\mathrm{R}$ using the MASS package (Venables and Ripley 2002).

\section{Allometric Scaling}

The influence of size on measures of abduction and abducted knee position was assessed using standard major axis (SMA) regression. Log-transformed femoral head diameter was selected as the size proxy because MacLatchy (1995) found that joint diameter is the functional scaling unit of the hip joint and a more appropriate size 
surrogate than other measures, particularly areal measures. In addition, males and females have equivalent scaling relationships between femoral head size and mass (Jungers 1991), minimizing the potential for estimation error. Angular data were not transformed but the femoral head sphere diameter was log-transformed prior to regression. Regression models were calculated using species sex-specific means, excluding Gorilla beringei, Alouatta caraya, and Pygathrix nemaeus due to the difficulties in assigning these taxa to a locomotor group.

SMA models were implemented in the smatr package of $R$ (Warton et al. 2012). SMA is preferable to least squares methods when testing the similarity of regression slopes between groups (Aiello 1992; Smith 2009). The suspensory and non-suspensory locomotor groups were tested for common slopes using a likelihood ratio test compared to a chi square distribution (Warton et al. 2006). If the slope tests were significantly different $(p \leq 0.05)$, individual regressions were fitted for the suspensory and nonsuspensory groups. A slope of 1 for linear and angular measures is considered isometric. If the isometric slope falls within the $95 \%$ confidence limits of the predicted slope, the two are not statistically different $(p \leq 0.05)$ (Goldman et al. 1990). Positive and negative allometry are indicated when the isometric slope falls beyond the $95 \%$ confidence intervals.

\section{Model validation}

In silico models of abduction and abducted knee position were compared to in vivo data in order to quantify how well the models represent the values from live animals. In vivo and in silico angle of abduction and abducted knee position were compared by percent difference, two-way ANOVA, and Tukey's post-hoc pairwise comparisons within taxa. 
In silico data were statistically compared to in vivo measures of angular abduction (Chapter 3) in order to evaluate the level of error in the modeling process, which is typically referred to as model validation (see review in Hutchinson 2011). In comparing in vivo and in silico data for angular abduction, taxa were compared at the genus level because taxa in the same genus are assumed to generally have the same locomotor adaptation (validation sample included in Table 4.13). The one exception to this was the mountain gorilla (G. beringei), which may differ substantially from the western lowland gorilla (G. gorilla) (see Chapter 2) in locomotor behaviors. In this case, comparisons between in vivo and in silico measures of abduction were restricted to $G$. gorilla.

In silico and in vivo data for abducted knee position were also compared. Whereas abduction is not expected to vary significantly within a genus (except perhaps in Gorilla), the abducted knee position could vary substantially when genera have different body masses. Because the abducted knee position has the potential to be greatly influenced by body size, validation statistics were considered at the species level instead.

\section{RESULTS}

\section{Differences between suspensory and non-suspensory groups in full extant sample}

\section{Femoral angular abduction}

Sex-specific species means for abduction are reported in Table 4.7. Suspensory taxa have significantly larger abduction (one-way ANOVA $p<0.01$ ) in silico than nonsuspensory taxa. Boxplots show that the two locomotor groups have substantial overlap between $35-55^{\circ}$ abduction (Figure 4.8). In particular, the low abduction ability of Gorilla 
gorilla results in suspensory taxa encompassing much of the range of non-suspensory taxa, although the suspensory taxa have increased abduction ability as a group compared to non-suspensory taxa (see also Figure 4.11).

\section{Knee position}

Sex-specific species means for abducted knee position are reported in Table 4.9. Suspensory taxa have significantly increased abducted knee positions (one-way ANOVA $\mathrm{p}<0.01)$ in silico than non-suspensory taxa. Boxplots show substantial overlap in abducted knee position between the locomotor groups, particularly between 175-225 $\mathrm{mm}$ from the midline (Figure 4.9).

\section{Classification accuracy}

\section{Femoral angular abduction}

Linear discriminant function analysis (DFA) was used to evaluate how well in silico abduction classifies group membership and at what level of specificity. This step is important for showing the reliability of locomotor predictions generated from models of abduction. DFA first compared locomotor groups (suspensory, non-suspensory), followed by very broad taxonomic groups with similar locomotor behaviors (hylobatids, Pongo, Gorilla gorilla, Pan, Ateles, Cebus, cercopithecoid). The DFA results show that broad locomotor categorization is a better classifier than the refined categories (Table 4.8). Overall, abduction ability cannot accurately predict taxonomic membership (59\% overall correct classification) but does accurately predict locomotor membership (80\% overall correct classification). The DFA results suggest that degree of abduction cannot classify an anthropoid beyond broad locomotor group (i.e., suspensory vs. nonsuspensory), and that different locomotor groups are more disparate in their abduction ability than the taxonomic groups. In addition to showing that locomotor categories are 
more accurate classifiers, the DFA results show that non-suspensory taxa are correctly classified with slightly higher frequency (83\%) than suspensory species (78\%), which is probably due to the low abduction values for G. gorilla.

\section{Knee position}

Discriminant function analysis (Table 4.8) shows that data for abducted knee position can be reasonably classified using broad locomotor categories (73\% correct classification in suspensory, $91 \%$ non-suspensory). As in abduction ability, sorting the in silico data by taxonomic unit returned low correct classification values (68\% correct).

\section{Allometric Scaling}

\section{Femoral angular abduction}

There is not a significant relationship between abduction and femoral head diameter ( $p>0.05$; see Figure 4.12). This is consistent with the live animal measures of abduction regressed on body size in Chapter 3.

\section{Knee position}

There is a significant relationship between the abducted knee position and femoral head diameter (Figure 4.12). Significant differences in slope were detected between locomotor groups $(p=0.025)$. The isometric slope is contained within the $95 \%$ confidence intervals for the predicted slope in non-suspensory primates (95\% Cl: $0.671-$ 1.080). The slope for non-suspensory primates is therefore not significantly different from isometry, and so it can be inferred that abducted knee position scales isometrically in this group. However, the isometric slope is not contained within the $95 \%$ confidence intervals for suspensory primates (95\% Cl: 0.508-0.728). The predicted slope for abducted knee position scales with negative allometry in suspensory taxa. 
It is important to note that hylobatids and Ateles have an abducted knee position that is larger than non-suspensory taxa of equivalent femoral head size (Figure 4.12). Although there are no extant large-bodied non-suspensory taxa to compare to the great apes, the negatively allometric slope in suspensory taxa indicates that great apes and similarly-sized non-suspensory taxa would converge on similar abducted knee positions. Why suspensory taxa scale with negative allometry in this measure has yet to be explored but probably relates to great apes having relatively shorter femora for their body size compared to cercopithecoids (see Discussion).

\section{Validation of in silico models with in vivo data}

\section{Femoral angular abduction}

In vivo and in silico measures of abduction were compared broadly and then compared at the taxonomic level. First, the two kinds of data were compared to each other, finding that the means of the in silico and in vivo data were not significantly different (ANOVA p=0.43, Table 4.10; see also Table 4.11 for means). This nonsignificant difference appeared to be a function of the in silico and in vivo data being differently distributed by locomotor category in vivo (e.g., in silico data was generally intermediate between the significantly different suspensory and non-suspensory in vivo data). Specifically, the in vivo data showed higher ranges of abduction for suspensory taxa and lower ranges of abduction for the non-suspensory taxa than was detected in silico. A two-way ANOVA comparing the interaction of locomotor group (suspensory versus non-suspensory) and data type (in silico versus in vivo) confirmed this observation, finding a significant interaction between these variables $(p<0.01$, Table 4.10). Examining the means of the data show that the in silico data overestimate range 
of in vivo abduction in non-suspensory primates, whereas the in silico data conservatively estimate range of in vivo abduction in suspensory primates (Table 4.11).

Comparisons were also made at the genus level. In vivo and in silico measures of abduction were found to overlap within each taxon (Figure 4.13), although there was only a small amount of overlap between in vivo and in silico data for Pan. Percent differences between data types by genus confirms this pattern on a finer scale (Table 4.13), although it is worth noting that percent differences maximize the appearance of differences between means with smaller values. For instance, for species with absolutely smaller angular abduction, a difference as small as $1^{\circ}$ will be a relatively larger percent difference compared to a species with an absolutely larger amount of angular abduction.

In addition, Tukey's honest significance test (Tukey's HSD) multiple comparisons between in silico and in vivo data types were performed between taxa (Table 4.14). Significant differences between means of data types were found in Pan, Macaca, Papio, and Ateles, consistent with the observations regarding percent differences.

\section{Knee position validation}

First, abducted knee position was broadly compared between in vivo and in silico data types. Means of the in silico and in vivo data were significantly different $(p<0.01$ in Table 4.12, see also Table 4.11 for means). Next, in vivo and in silico data were divided into their locomotor groups and a two-way ANOVA was used to test for a significant interaction between locomotor group and data type. The two-way ANOVA found a significant interaction between these variables $(p<0.01$, Table 4.12). A comparison of the mean values shows that in silico data for suspensory primates conservatively 
estimates knee position, although in vivo and in silico means for non-suspensory taxa are virtually the same (Table 4.11).

The abducted knee position was also compared at the species level. The in vivo and in silico data for knee position were found to overlap when visually examined (Figure 4.14). The species-specific means between the two data types differ in some species, however. Percent differences in mean knee position were relatively large in Pan (female $=15 \%$, male=21\%), Symphalangus (female=20\%, male=23\%), and Cebus (male $=20 \%$ ) (Table 4.13). However, as previously noted, there are certain limitations to examining differences by percentage. A more specific comparison between the two data types are through Tukey's HSD pairwise comparisons between data types for each species (Table 4.15). All taxa were found to have means that were not statistically different between in silico and in vivo data for knee position, except Pan troglodytes. Pan troglodytes was statistically significant $(p<0.01$, Table 4.15$)$. It is probable that this difference in P. troglodytes is responsible for the difference between in silico and in vivo suspensory means in Table 4.11. However, in silico and in vivo knee position show a very strong relationship in the other species, indicating a high correspondence between in vivo and in silico data for knee position.

\section{DISCUSSION}

As anticipated, the in silico models indicate that there are significant differences between suspensory and non-suspensory species, particularly in regards to the more highly suspensory species (Pongo, Pan, hylobatids) (see Figure 4.10). Interestingly, Gorilla has a lower simulated range of angular abduction than other suspensory species and overlaps with non-suspensory species in this measure. In particular, Gorilla beringei has a range of abduction between $21-39^{\circ}$ (Table 4.7, Figure 4.11), which is at the low 
range even for non-suspensory monkeys. Of particular relevance here is that gorillas are the largest of the extant primates, with male G. gorilla and G. beringei averaging 170 $\mathrm{kg}$ and $163 \mathrm{~kg}$, respectively (Smith and Jungers 1997). It is suggested here, as suggested by others (Rubin and Lanyon 1982; Biewener 1983; Rubin and Lanyon 1984; Alexander 1985; Polk et al. 2009; Zihlman et al. 2011), that very large-bodied species such as Gorilla may operate their joints in a narrower range of positions and/or at slower speeds in order to maintain acceptable joint safety factors. However, both species are similarly large and so this does not fully explain why $G$. beringei has an even lower range of abduction than G. gorilla. These differences probably relate to different locomotor behaviors and associated habitual limb postures. As discussed in Chapter 2, Gorilla beringei is substantially more terrestrial than any of the suspensory species (Schaller 1963; Reynolds 1965; Doran 1996; Doran and McNeilage 1998), including its co-generer Gorilla gorilla (Doran and Hunt 1994; Remis 1995; Doran 1997; Remis 1998). Gorilla beringei uses terrestrial quadrupedalism for $97 \%$ of locomotor behaviors (Doran 1996) whereas Gorilla gorilla is only quadrupedal 64\% of the time (Hunt 2004) (see Table 2.1).

Abducted knee posture is a proxy for the hindlimb spatial envelope, or range of foot positions relative to the body (Figure 4.15), and is the additive result of hip mobility, body size, and morphology. Large animals have absolutely long thighs and so, not surprisingly, the in silico models show that range of abducted knee positions are significantly larger in suspensory primates, especially in the large-bodied great apes. This finding was anticipated based on results in Chapter 3, but this chapter shows that the abducted knee position is influenced by morphology in addition to body size and range of motion. The importance of morphology is inferred because the abducted knee 
position does not scale isometrically in suspensory taxa, with great apes having a lower value than would be predicted based on size alone. This almost certainly results from the proportions of the hindlimbs in great apes, specifically that great apes have relatively short femora for their size (Jungers and Susman 1984; Jungers 1985).

This chapter also confirms that certain taxa have similar abducted knee positions but different ranges of abduction, indicating body size is an important factor in abducted knee position. A clear example of this is for instance Pongo and Gorilla (Figure 4.15). Although Gorilla and Pongo have the ability to position their knee equally far lateral relative to the midline of the body (Figure 4.9), the angle at which they do this is different (Figure 4.8), resulting in somewhat different functional abilities. Although the range of knee positions possible conveys basic information about locomotor behavior (e.g., "suspensory" vs. "non-suspensory") and what range of supports are directly available to an animal, abducted knee position cannot provide information about the more subtle aspects of locomotor behavior that distinguish different taxa. That is, Gorilla and Pongo are quite different in their specific locomotor adaptations and hindlimb capabilities but this is not detected from abducted knee position alone. In short, the spectrum of locomotor behaviors known to exist in anthropoids (see Chapter 2) is not distinguished in this metric without additional information about range of abduction.

The findings in this study are compatible with those reported by MacLatchy (MacLatchy 1995; 1996; MacLatchy and Bossert 1996), who modeled joint mobility in Pongo pygmaeus, Pan troglodytes, Mandrillus sphinx, and Macaca fascularis. In abduction during extension ${ }^{10}$, MacLatchy found significantly different abduction in $M$.

\footnotetext{
${ }^{10}$ MacLatchy's (1995) dissertation simulated abduction from an extended posture (parallel or $0^{\circ}$ to the ischial ramus) and a flexed posture $\left(135^{\circ}\right.$ to the ischial ramus). Her subsequent works $(1996,1998)$ also reference a "neutral posture", when the fovea capitis is centered in the acetabular fossa and the shaft of the femur is flexed $90^{\circ}$ to the ischial ramus. The neutral posture used in MacLatchy's study is the most comparable to
} 
fascicularis and M. sphinx with abduction in $P$. troglodytes and $P$. pygmaeus (1995; 1996). In abduction during flexion, MacLatchy was unable to detect significant differences between $M$. fascicularis, $M$. sphinx, and $P$. troglodytes but did find $P$. pygmaeus to have a significantly greater range of motion $(1995 ; 1996)$. Overall, her work indicated there is a spectrum of mobility, with Old World monkeys having the lowest range of motion and $P$. pygmaeus having the greatest, with $P$. troglodytes being intermediate between the two. It was previously unclear whether the patterns observed in MacLatchy's study were related to a limited taxonomic sample, phylogenetic influences, and/or allometric effects, but this work demonstrates that hip abduction varies with locomotor behavior. The spectrum of hip mobility evinced in MacLatchy's work is present here, with Pongo having the greatest range of abduction in the hominoids, followed by hylobatids and Pan, and Gorilla gorilla being lower and overlapping with some non-suspensory monkeys (Figure 4.13).

This study can be more specific than MacLatchy's study regarding hip mobility in cercopithecoids and clarifies some of her findings. This study sampled a much larger number of cercopithecoids and found that most colobine monkeys and macaques generally have increased range of abduction than terrestrial cercopithecines (Papio, Theropithecus, Erythrocebus). This finding presumably reflects the increased arboreality of colobines and macaques. Macaca fascicularis is unusual among cercopithecine monkeys in more frequently being arboreal (Rodman 1979; Cannon and Leighton 1994) and is apparently capable of more mobility than many other cercopithecine monkeys, explaining why MacLatchy's study did not detect significant differences between Pan and Macaca when abducting from flexed hip postures.

the shaft orientation in this study. Unfortunately, very limited data are provided on the neutral posture in anthropoids (see MacLatchy 1996) but it is said to yield the same results as the flexed posture. 
Cebus, Alouatta, and Ateles show that increased arboreal versatility is also associated with an increased range of abduction in the platyrrhines. Cebus has the smallest range of abduction, followed by Alouatta, and with Ateles having the largest range of the three platyrrhines (see Figure 4.8). The ranges for Cebus and Ateles are consistent with the behavioral data for these taxa (see Chapter 2). The behavioral data for Alouatta emphasized arboreal quadrupedalism (Grand 1968; Mendel 1976; Fleagle and Mittermeier 1980) but there are reports that Alouatta occasionally uses prehensile tail-assisted suspensory behaviors (Grand 1968; Fleagle and Mittermeier 1980; Ybarra 1984; Gebo 1992; Prates and Bicca-Marques 2008), making a discrete locomotor classification difficult for this genus. However, range of abduction for Alouatta falls between quadrupedal Cebus and frequently suspensory Ateles, which seems to fit with the intermediate behavioral data for Alouatta. Not surprisingly, the influence of higher abduction ability in Ateles is reflected in the abducted knee position. Ateles has a larger span at the knee than Alouatta (see Figure 4.9), despite both taxa having similar reported body masses ( $\sim 6 \mathrm{~kg}$, Smith and Jungers 1997), and both Ateles and Alouatta have a larger span at the knee than Cebus.

The results for cercopithecoids show a few unexpected results. Asian colobine Pygathrix nemaeus has been reported to use some suspensory behaviors in captive settings (Workman and Covert 2005; Wright et al. 2008) but the hip morphology does not necessarily promote high levels of hip abduction as would be expected in a suspensory species. Instead, $P$. nemaeus has hip abduction consistent with most other non-suspensory arboreal cercopithecoids. This would suggest that either additional behavioral data is needed on free-ranging $P$. nemaeus, or that the moderate level of hip mobility is adequate and/or necessary for locomotor behaviors used by $P$. nemaeus. Colobus guereza, however, has somewhat low range of abduction compared to the 
arboreal cercopithecoids (e.g., Nasalis, Rhinopithecus, Trachypithecus, Piliocolobus) and falls within the range of the more terrestrial cercopithecoids (e.g., Papio, Erythrocebus). Although there is a spectrum of abduction within the cercopithecoids, one possibility raised by these taxa is that monkeys, perhaps even arboreal ones, need a more limited range of abduction for their quadrupedal locomotor behaviors. Lateral joint excursions might be detrimental to powerful flexion-extension movements at the hip, and so taxa that do propulsive leaping (e.g., Colobus) or cover large distances by moving terrestrially (e.g., Papio, Theropithecus) might be especially likely to have less abduction possible at their hip joints. Additional work evaluating morphological and biomechanical trade-offs associated with different hindlimb usage are necessary to test this hypothesis.

This study found that in silico measures of abduction and abducted knee position can be used to predict locomotor category with some accuracy, which is an important criterion for reconstructing locomotor behaviors in fossil taxa. Discriminant function analyses were used to predict categorical group membership (suspensory vs. nonsuspensory) from the measures of abduction and abducted knee position. In silico measures of abduction correctly predicted locomotor category $80 \%$ of the time in DFA, and knee position correctly predicted locomotor category $81 \%$ of the time. However, the models of abduction and abducted knee position cannot correctly predict taxonomic groups that broadly correspond to more refined locomotor classifications (i.e., specialized brachiation in hylobatids, terrestrial knuckle-walking in Gorilla and Pan, quadrumanous anti-pronograde clambering in Pongo, clambering and semi-brachiation in Ateles, and quadrupedalism in Cebus). The DFA results found a low overall prediction accuracy for abduction (59\% correct classification) and abducted knee position (68\% correct classification) when divided into smaller groups. These results 
make it seem unlikely that the in silico models can be used to achieve higher levels of specificity beyond the broad locomotor categories (i.e., suspensory vs. non-suspensory).

The validity of the interpretations garnered from this model entirely depends on how well the model matches some form of empirical data. Quantifying how closely a model matches empirical data is referred to as model "validation" (Hutchinson 2011), and this study has given particular attention to quantifying the relationship between models of hip abduction and abduction in living anthropoids. This work does not intend to prove that the modeling approach is exact, as models can only approximate reality and many validation tests produce extremely high error rates (review in Hutchinson 2011). The comparisons between the two kinds of data highlight certain differences that exist between the two samples and it is imperative to be aware of how the two kinds of data differ (discussed below). Fortunately, the in silico and live animal data quantified for this study have a predictable relationship, suggesting that the modeling approach outlined here has utility for paleobiological reconstructions of hip joint abduction in extinct anthropoids.

Angular abduction displays differences between the in silico and in vivo approaches in certain taxa. The two forms of data are congruent in Symphalangus, Pongo, Gorilla, and Cebus in abduction, with substantial overlap between in vivo and in silico data (Figure 4.13) and non-significant species-specific pairwise comparisons between data (Table 4.14). It is more difficult to evaluate Trachypithecus, Colobus and Hylobates due to sample sizes, but the limited in silico and in vivo data also overlap (Figure 4.13). The in vivo and in silico data for Macaca, Papio, Ateles, and Pan display little overlap (Figure 4.13) and are significantly different in pairwise comparisons (Table 4.14). This shows that there is some variability in which species will deviate in silico 
from the values expected based on the live animal data; differences between the two forms of data are not limited to a single locomotor group or a single species.

Despite some of these observed differences between the in silico and in vivo data for angular abduction, the way the data differ are consistent. Modeled abduction is underestimated in suspensory species compared to live animal data, and overestimated in the non-suspensory species. Because the models of abduction are based on positions assumed during loading, it might be expected that the models would underestimate passive range of motion, although this does not explain why nonsuspensory models overestimate abduction. Differences between the two locomotor groups are diminished in silico but predictions that fall in the suspensory range are likely to conservatively estimate the true range of abduction whereas predictions that are in the range of non-suspensory taxa are likely to be an overestimate of abduction ability.

The abducted knee position in silico closely tracks the in vivo data at the species level. This result indicates that the functional outcome of hip mobility-the abducted knee position-is approximated with a high degree of accuracy in the modeling approach, despite some differences between the two data types observed in angle of abduction. Pairwise comparisons show that only Pan troglodytes is significantly different in abducted knee position between data types (Table 4.15). However, none of the other comparisons between in silico and in vivo data were significantly different and there is nearly complete overlap between the two data for the other species (Figure 4.14). Because the models of hip abduction during loading closely approximate the abducted knee position measured in anesthetized primates, this suggests that the modeling approach developed here actually is converging on the overall hindlimb capabilities of the animals. 
Of course, it is important to keep in mind that the models of hip abduction are meant to represent the most abducted position of the joint under loading, whereas the live animal data are the maximum positions allowed by the joint when overlying soft tissues are present. This is a fundamental distinction to be made between these two kinds of data. Models of hip abduction are limited by the bony morphology, and so the bony morphology clearly determines the range of abduction in these models. Hip mobility is not primarily determined by bony morphology in living animals, however, and is instead limited by the surrounding soft tissue structures. When soft tissues are not present, the bones of the non-suspensory taxa overestimate the range of abduction at the joint, indicating that the overlying soft tissues structures are important for limiting joint mobility in these taxa. In suspensory taxa, the models of maximum hip abduction underestimate the range of abduction possible.

The differences between live animal and digital measures of abduction in this study are clarified by MacLatchy's results for strepsirrhines, which suggest that models of maximum hip abduction usually underestimate passive range of abduction. Although limited to strepsirrhines, MacLatchy (1995) also found that angle of abduction in digital models was typically less than passive abduction measured in vivo. MacLatchy (1995) measured a small number of strepsirrhines both in silico (Microcebus $n=8$, Galago $n=8$, Cheirogaleus n=3, Periodicticus $\mathrm{n}=5$ ) and in vivo (Microcebus $\mathrm{n}=1$, Galago $\mathrm{n}=2$, Cheirogaleus $n=2$, Periodicticus $n=2$ ). Maximum angle of abduction during flexion and extension was compared between digital and live animal data, although the small sample limited the statistical tests that could be performed. All in vivo data were within a single standard deviation of the mean for maximum angles of abduction during flexion, and the in vivo angles were usually larger than the mean in the digital models (see 
MacLatchy 1995, p. 122). Similarly, maximum angles of abduction during extension were usually within a single standard deviation of the in silico means, although one Galago and one Cheirogaleus individual were closer to two standard deviations above the in silico means (see MacLatchy 1995, p. 123). MacLatchy's results suggest that passive abduction measured in strepsirrhines slightly over-estimates abduction measured in silico, similar to what was observed in suspensory anthropoids in this study. In short, the digital modeling technique used by MacLatchy (1995) and further developed in this study conservatively estimates the total range of femoral abduction in strepsirrhines, hominoids, and Ateles. What remains unexplained is why this study overestimates abduction in non-suspensory cercopithecoids, particularly for Macaca.

There are several possibilities for why live animal abduction measurements for Macaca are lower than predicted by the models. An untested possibility which cannot be currently excluded is that abduction measured in the in vivo macaque sample does not accurately reflect wild-shot range of motion. The macaques sampled in this study (Chapter 3) were housed in cages and not allowed to roam like the Papio sample, which was housed in a large outdoor colony. However, the possibility that the in vivo measures are under-estimates is somewhat unlikely given the other similarly low values found in the other cercopithecoids. In addition, past studies on anthropoid joint mobility have found captive animals to have larger range of motion than wild counterparts (Turnquist 1983; 1985). Although it seems unlikely that captive macaques would have a substantially reduced range of abduction, the influence of caging could be tested by digitally modeling range of abduction using models derived from captive macaques.

A more likely possibility proposed here is that external (lateral) rotation is limited in vivo by soft tissues in non-suspensory species but these limits on movement are 
absent from the digital model. The work in Chapter 3 supported previous inferences made by Stern and Larson (1993) that highly abducted hip postures in primates involve concomitant external rotation. High levels of external rotation were found to be an important factor in passive range of hip abduction in suspensory taxa, and it was hypothesized that external rotation in vivo was limited by soft tissue structures surrounding the hip joint in non-suspensory taxa. In particular, interspecific differences in the strength of hip joint capsule ligaments, the size of the acetabular labrum, and differences in muscle extensibility, tone, and attachments were hypothesized to affect passive range of external rotation (Chapter 3). Because the models here cannot incorporate soft-tissue limits on external rotation, abduction simulations in the nonsuspensory taxa may have had a higher level of external rotation occurring in silico than would have occurred in vivo. If this occurred, the increased external rotation could have facilitated an increased level of abduction in the non-suspensory models.

\section{CONCLUSIONS}

Extant suspensory taxa have a significantly greater range of hip joint abduction in silico than non-suspensory taxa. Importantly, the inclusion of Ateles and Cebus shows that the patterns observed within suspensory hominoids and non-suspensory cercopithecoids are not just related to phylogeny. Discriminant function analysis can classify taxa as suspensory or non-suspensory using in silico estimates of abduction, although given the overlap in degrees of abduction between suspensory and nonsuspensory taxa there must be some caution when estimates are intermediate between the two groups. Only fossil taxa that are found well within the suspensory $\left(>55^{\circ}\right)$ or nonsuspensory $\left(<35^{\circ}\right)$ ranges can be easily attributed to one of these locomotor groups. The abducted knee position is significantly different as well but, as in degrees of 
abduction, there is some overlap between locomotor groups. The abducted knee position is related to body size and so this metric is most appropriately considered along with hip abduction (degrees) in order to make inferences about arboreal versatility in fossil anthropoids.

There is a predictable relationship between hip joint abduction and knee position modeled in silico and that measured in vivo. The in silico abduction simulations behave in a predictable way relative to the in vivo range of abduction, and there is an even stronger relationship between data in reference to knee position. It should be emphasized that in vivo angular abduction is the maximum range allowed by the joint when overlying soft tissues are present, whereas the models are built on criteria about how joints are expected to operate during conditions of loading. Given the inherent difference in what is being measured, we would not expect one-to-one correspondence between the two kinds of data. However, the results present a clear hypothetical framework for interpreting in silico abduction estimates derived for fossil taxa: fossil estimates that are consistent with extant suspensory taxa are conservative estimates of actual range of abduction allowed by the joint, whereas fossil estimates consistent with non-suspensory taxa are probably high estimates of possible range of angular abduction. 
Table 4.1 Extant laser scan and microscribe landmark sample $(n=252)$

\begin{tabular}{|c|c|c|c|}
\hline Taxon & $\hat{o}(q)$ & Locomotion & Museum \\
\hline \multicolumn{4}{|l|}{ platyrrhines (n=33) } \\
\hline Ateles belzebuth & $0(2)$ & S & AMNH \\
\hline Ateles fusciceps & $2(4)$ & S & USNM \\
\hline Ateles geoffroyi & $0(3)$ & $S$ & USNM \\
\hline Ateles paniscus & $1(1)$ & S & ZMA, AMNH \\
\hline Alouatta caraya & $5(5)$ & -- & AMNH \\
\hline $\begin{array}{l}\text { Cebus apella } \\
\text { colobines }(n=52)\end{array}$ & $3(6)$ & $\mathrm{N}$ & AMNH, USNM, ZMA \\
\hline$\overline{\text { Colobus guereza }}$ & $2(3)$ & $\mathrm{N}$ & USNM, KNM \\
\hline Nasalis larvatus & $8(9)$ & $\mathrm{N}$ & MCZ, USNM, ZSM \\
\hline Pilocolobus badius & $0(4)$ & $\mathrm{N}$ & PCM \\
\hline Rhinopithecus roxellana & $1(2)$ & $\mathrm{N}$ & USNM, AMNH \\
\hline Trachypithecus cristatus & $8(8)$ & $\mathrm{N}$ & MCZ, ZSM, USNM, ZMA \\
\hline $\begin{array}{l}\text { Pygathrix nemaeus } \\
\text { cercopithecines }(n=55)\end{array}$ & $5(2)$ & -- & USNM, MCZ, AMNH \\
\hline Cercopithecus mitis & $4(4)$ & $N$ & USNM \\
\hline Erythrocebus patas & $3(2)$ & N & AMNH, USNM, ZSM \\
\hline Macaca fascicularis & $4(6)$ & $\mathrm{N}$ & MCZ, USNM \\
\hline Macaca nemestrina & $1(5)$ & $\mathrm{N}$ & MCZ \\
\hline Papio cynocephalus & $1(7)$ & & KNM, USNM \\
\hline Papio anubis & $4(5)$ & $\mathrm{N}$ & USNM \\
\hline $\begin{array}{l}\text { Theropithecus gelada } \\
\text { hominoids ( } \mathrm{n=112} \text { ) }\end{array}$ & $3(6)$ & N & UZIA, USNM, ZMA \\
\hline Hylobates lar & $7(10)$ & S & ZSM, MCZ \\
\hline Symphalangus syndactylus & $10(11)$ & $\mathrm{S}$ & ZSM, USNM, ZMA, AMNH \\
\hline Pongo pygmaeus & $5(10)$ & S & CMNH, USNM, MCZ, ZMA \\
\hline Pongo abelii & $3(3)$ & S & USNM, MCZ \\
\hline Gorilla gorilla & $10(8)$ & $\mathrm{S}$ & CMNH, USNM \\
\hline Gorilla beringei & $3(3)$ & -- & USNM \\
\hline Pan troglodytes & 8 (10) & $S$ & CMNH, USNM \\
\hline Pan paniscus & $5(6)$ & $\mathrm{S}$ & MRAC \\
\hline
\end{tabular}

Locomotor abbreviations: S, suspensory; N, non-suspensory; dashes (--), unassigned locomotor category. Institutional abbreviations are listed in text and Appendix $\mathrm{C}$. 
Table 4.2 Microscribe landmark coordinates

\begin{tabular}{lll}
\hline Landmark & Location & Used for \\
\hline 1 & Left posterior & virtual alignment of scanned \\
2 & Left anterior & virtual alignment of scanned \\
3 & Left dorsal & virtual alignment of scanned \\
4 & Left acetabular & virtual alignment of scanned \\
5 & Left ischial spine & virtual alignment of scanned \\
6 & Caudal pubic & midline plane \\
7 & Cranial pubic & midline plane \\
8 & Caudal S1 & midline plane \\
9 & Caudal S2 & midline plane \\
10 & Caudal S3 & midline plane \\
11 & Dorsal cranial & lumbosacral plane \\
12 & Ventral cranial & lumbosacral plane \\
13 & Right cranial border & lumbosacral plane \\
14 & Left cranial border & lumbosacral plane
\end{tabular}


Table 4.3 Intraobserver mean deviation $(\mathrm{mm})$ and error (RMSE) across landmarks

\begin{tabular}{ccc}
\hline & Mean Deviations & RMSE \\
\hline Mean & 0.35 & 0.89 \\
Max & 1.67 & 1.30 \\
Min & 0.00 & 0.56
\end{tabular}


Table 4.4 Interlandmark distances used in pelvis articulation error study

\begin{tabular}{ll}
\hline \multicolumn{2}{c}{ Interlandmark Distances } \\
\hline Distance 1 & Left ASIS to dorsal S1 \\
Distance 2 & Left ASIS to inferior S1 \\
Distance 3 & Left acetabular center to dorsal S1 \\
Distance 4 & Left acetabular center to inferior S1
\end{tabular}


Table 4.5 Interlandmark distances $(\mathrm{mm})$ and mean deviations $(\mathrm{mm})$

\begin{tabular}{crrrr}
\hline & Distance 1 & Distance 2 & Distance 3 & Distance 4 \\
\hline Interlandmark Distances & & & & \\
Max & 141.9 & 136.73 & 151.68 & 127.62 \\
Min & 141.01 & 135.45 & 150.52 & 126.16 \\
\%Difference* & $0.63 \%$ & $0.94 \%$ & $0.77 \%$ & $1.15 \%$ \\
Mean Deviations & & & & \\
Mean & 0.26 & 0.4 & 0.4 & 0.4 \\
Max & 0.52 & 0.64 & 0.72 & 0.78 \\
Min & 0.09 & 0.15 & 0.21 & 0.08
\end{tabular}

*Percent difference represents the difference between the maximum and minimum interlandmark distances. 
Table 4.6 Differences in 5 repeated trials of best-fit diaphyseal vectors for Papio and Pan specimens.

\begin{tabular}{|c|c|c|c|}
\hline \multirow[t]{2}{*}{ Specimen } & \multicolumn{3}{|c|}{ Difference in orientation between vectors } \\
\hline & Maximum & Minimum & Mean \\
\hline Pan troglodytes (CMNH B1056 О人) & 0.52 & 0.07 & 0.26 \\
\hline Pan troglodytes (CMNH B1758 ડ̄) & 0.47 & 0.04 & 0.25 \\
\hline Pan troglodytes (CMNH B1770 q) & 0.45 & 0.11 & 0.26 \\
\hline Pan troglodytes (CMNH B1723 ó) & 1.16 & 0.03 & 0.52 \\
\hline Pan troglodytes (CMNH B1755 o) & 0.34 & 0.01 & 0.18 \\
\hline Papio anubis (NMNH 483234 ठ઼) & 0.43 & 0.10 & 0.24 \\
\hline Papio anubis (NMNH 394989 ठో) & 0.96 & 0.16 & 0.53 \\
\hline Papio anubis (NMNH 395441 ㅇ) & 0.39 & 0.03 & 0.19 \\
\hline Papio anubis (NMNH 397476 ó) & 0.35 & 0.08 & 0.18 \\
\hline Papio anubis (NMNH 395438 of) & 0.48 & 0.02 & 0.29 \\
\hline
\end{tabular}

Vectors from 5 repeated trials per individual compared equaling 10 comparisons per specimen. 
Table 4.7 Sex-specific descriptive statistics for abduction (in silico)

\begin{tabular}{|c|c|c|c|c|}
\hline & \multirow[b]{2}{*}{ Sex } & \multirow[b]{2}{*}{$\mathrm{n}$} & \multicolumn{2}{|c|}{ Abduction $\left({ }^{\circ}\right)$} \\
\hline & & & Mean \pm SD & Max, Min \\
\hline \multirow[t]{2}{*}{ Pan troglodytes } & $\mathrm{F}$ & 10 & $54.96 \pm 5.25$ & $64.70,47.55$ \\
\hline & $M$ & 8 & $49.70 \pm 6.71$ & $61.99,42.06$ \\
\hline \multirow[t]{2}{*}{ Pan paniscus } & $\mathrm{F}$ & 6 & $60.35 \pm 2.10$ & $62.67,57.29$ \\
\hline & $M$ & 5 & $62.89 \pm 3.31$ & $66.76,59.56$ \\
\hline \multirow[t]{2}{*}{ Gorilla gorilla } & $\mathrm{F}$ & 8 & $50.02 \pm 4.61$ & $55.90,41.57$ \\
\hline & $M$ & 10 & $39.69 \pm 6.40$ & $48.73,29.83$ \\
\hline \multirow[t]{2}{*}{ Gorilla beringei } & $\mathrm{F}$ & 3 & $33.74 \pm 4.63$ & $38.81,29.73$ \\
\hline & $M$ & 3 & $26.76 \pm 7.38$ & $35.12,21.17$ \\
\hline \multirow[t]{2}{*}{ Pongo pygmaeus } & $\mathrm{F}$ & 10 & $75.66 \pm 8.83$ & $86.24,60.34$ \\
\hline & $M$ & 5 & $71.44 \pm 1.68$ & $73.30,69.00$ \\
\hline \multirow[t]{2}{*}{ Pongo abelii } & $\mathrm{F}$ & 3 & $75.30 \pm 5.36$ & $81.49,72.04$ \\
\hline & $M$ & 3 & $75.11 \pm 4.40$ & $80.15,72.06$ \\
\hline \multirow[t]{2}{*}{ Symphalangus syndactylus } & $\mathrm{F}$ & 11 & $57.12 \pm 5.57$ & $63.80,45.50$ \\
\hline & $M$ & 10 & $56.45 \pm 7.56$ & $66.70,43.82$ \\
\hline \multirow[t]{2}{*}{ Hylobates lar } & $\mathrm{F}$ & 10 & $60.16 \pm 7.26$ & $68.00,47.38$ \\
\hline & $M$ & 7 & $55.50 \pm 8.50$ & $67.27,44.58$ \\
\hline \multirow[t]{2}{*}{ Colobus guereza } & $\mathrm{F}$ & 3 & $34.16 \pm 7.19$ & $38.77,25.87$ \\
\hline & $M$ & 2 & $30.02 \pm 4.44$ & $33.16,26.88$ \\
\hline \multirow{2}{*}{ Nasalis larvatus } & $\mathrm{F}$ & 9 & $44.76 \pm 5.75$ & $55.47,35.59$ \\
\hline & $M$ & 8 & $46.12 \pm 5.71$ & $52.59,36.74$ \\
\hline Piliocolobus badius & $\mathrm{F}$ & 4 & $39.86 \pm 6.18$ & $48.58,34.49$ \\
\hline \multirow[t]{2}{*}{ Rhinopithecus roxellana } & $\mathrm{F}$ & 2 & $40.45 \pm 0.08$ & $40.51,40.40$ \\
\hline & M & 1 & 42.87 & NA \\
\hline \multirow[t]{2}{*}{ Trachypithecus cristatus } & $\mathrm{F}$ & 8 & $46.38 \pm 4.89$ & $51.81,39.97$ \\
\hline & $M$ & 8 & $44.07 \pm 5.59$ & $53.43,37.89$ \\
\hline \multirow[t]{2}{*}{ Pygathrix nemaeus } & $\mathrm{F}$ & 2 & $46.65 \pm 4.11$ & $49.56,43.75$ \\
\hline & $M$ & 5 & $52.09 \pm 6.16$ & $62.56,47.63$ \\
\hline \multirow[t]{2}{*}{ Cercopithecus mitis } & $\mathrm{F}$ & 4 & $37.97 \pm 6.60$ & $46.91,32.01$ \\
\hline & M & 4 & $38.81 \pm 6.79$ & $48.90,34.19$ \\
\hline \multirow{2}{*}{ Erythrocebus patas } & $\mathrm{F}$ & 2 & $36.27 \pm 3.42$ & $38.69,33.85$ \\
\hline & M & 3 & $22.14 \pm 6.49$ & $29.57,17.64$ \\
\hline \multirow[t]{2}{*}{ Macaca fascicularis } & $\mathrm{F}$ & 6 & $45.95 \pm 5.58$ & $52.51,39.05$ \\
\hline & M & 4 & $44.22 \pm 1.83$ & $46.52,42.34$ \\
\hline \multirow[t]{2}{*}{ Macaca nemestrina } & $\mathrm{F}$ & 5 & $48.68 \pm 5.58$ & $53.70,40.77$ \\
\hline & $M$ & 1 & 56.52 & NA \\
\hline \multirow[t]{2}{*}{ Papio cynocephalus } & $\mathrm{F}$ & 7 & $37.23 \pm 6.54$ & $48.11,29.71$ \\
\hline & M & 1 & 36.07 & NA \\
\hline Papio anubis & $\mathrm{F}$ & 5 & $32.26 \pm 8.04$ & $43.53,25.52$ \\
\hline & $M$ & 4 & $37.99 \pm 2.51$ & $41.46,35.77$ \\
\hline Theropithecus gelada & $\mathrm{F}$ & 6 & $39.38 \pm 7.52$ & $49.87,28.31$ \\
\hline & $M$ & 3 & $38.48 \pm 6.80$ & $45.15,31.55$ \\
\hline Ateles belzebuth & $\mathrm{F}$ & 2 & $60.55 \pm 0.63$ & $60.99,60.11$ \\
\hline Ateles fusciceps & $\mathrm{F}$ & 4 & $50.12 \pm 8.79$ & $62.49,42.88$ \\
\hline & $M$ & 2 & $45.14 \pm 4.11$ & $48.04,42.24$ \\
\hline Ateles geoffroyi & $\mathrm{F}$ & 3 & $52.71 \pm 0.92$ & $53.41,51.66$ \\
\hline Ateles paniscus & M & 3 & $54.95 \pm 10.48$ & $62.27,42.95$ \\
\hline Alouatta caraya & $\mathrm{F}$ & 5 & $53.23 \pm 7.53$ & $60.98,42.16$ \\
\hline & $M$ & 5 & $48.46 \pm 1.93$ & $50.38,45.63$ \\
\hline Cebus apella & $\mathrm{F}$ & 6 & $42.48 \pm 5.31$ & $48.19,34.08$ \\
\hline & $M$ & 3 & $41.18 \pm 4.87$ & $46.63,37.24$ \\
\hline
\end{tabular}


Table 4.8 Percent correct classification in in silico models using discriminant function analysis

\begin{tabular}{lcr}
\hline Locomotor Group & $\begin{array}{c}\text { Abduction } \\
\text { (degrees) }\end{array}$ & $\begin{array}{r}\text { Abducted knee position } \\
(\mathrm{mm})\end{array}$ \\
\hline Suspensory $(\mathrm{n}=120)$ & $78.33 \%$ & $72.50 \%$ \\
Non-suspensory $(\mathrm{n}=109)$ & $82.57 \%$ & $90.83 \%$ \\
Total $(\mathrm{n}=229)$ & $80.35 \%$ & $81.22 \%$ \\
\hline & Abduction & Abducted knee position \\
Taxonomic Group & (degrees) & $84.21 \%$ \\
\hline hylobatid $(\mathrm{n}=38)$ & $63.16 \%$ & $0.00 \%$ \\
Pongo $(\mathrm{n}=21)$ & $90.48 \%$ & $61.11 \%$ \\
G. gorilla $(\mathrm{n}=18)$ & $0.00 \%$ & $93.10 \%$ \\
Pan $(\mathrm{n}=29)$ & $0.00 \%$ & $0.00 \%$ \\
Ateles $(\mathrm{n}=14)$ & $0.00 \%$ & $33.33 \%$ \\
Cebus $(\mathrm{n}=9)$ & $0.00 \%$ & $82.00 \%$ \\
cercopithecoid $(\mathrm{n}=100)$ & $93.00 \%$ & $67.69 \%$ \\
Total $(\mathrm{n}=229)$ & $59.39 \%$ &
\end{tabular}

Alouatta caraya, Gorilla beringei, and Pygathrix nemaeus were excluded from the comparisons due to uncertain locomotor classification. 
Table 4.9 Sex-specific descriptive statistics for abducted knee position (in silico)

\begin{tabular}{|c|c|c|c|c|}
\hline & \multirow[b]{2}{*}{ Sex } & \multirow[b]{2}{*}{$\mathrm{n}$} & \multicolumn{2}{|c|}{ Abducted knee position (mm) } \\
\hline & & & Mean \pm SD & Max, Min \\
\hline \multirow[t]{2}{*}{ Pan troglodytes } & $\mathrm{F}$ & 10 & $301.59 \pm 19.77$ & $337.35,272.70$ \\
\hline & M & 8 & $298.29 \pm 20.16$ & $335.03,277.31$ \\
\hline \multirow[t]{2}{*}{ Pan paniscus } & $\mathrm{F}$ & 6 & $318.77 \pm 11.07$ & 330.83, 305.09 \\
\hline & M & 5 & $310.23 \pm 10.32$ & $322.84,294.51$ \\
\hline \multirow[t]{2}{*}{ Gorilla gorilla } & $\mathrm{F}$ & 8 & $324.02 \pm 19.69$ & $358.09,298.97$ \\
\hline & M & 10 & $353.31 \pm 24.97$ & $393.74,310.32$ \\
\hline \multirow[t]{2}{*}{ Gorilla beringei } & $\mathrm{F}$ & 3 & $274.18 \pm 10.85$ & $280.75,261.66$ \\
\hline & M & 3 & $304.34 \pm 33.53$ & $336.06,269.25$ \\
\hline \multirow[t]{2}{*}{ Pongo pygmaeus } & $\mathrm{F}$ & 10 & $293.87 \pm 17.41$ & $329.04,267.54$ \\
\hline & M & 5 & $332.15 \pm 10.54$ & $345.61,319.46$ \\
\hline \multirow[t]{2}{*}{ Pongo abelii } & $\mathrm{F}$ & 3 & $285.63 \pm 4.02$ & $288.49,281.04$ \\
\hline & M & 3 & $353.84 \pm 19.95$ & $376.66,339.71$ \\
\hline \multirow[t]{2}{*}{ Symphalangus syndactylus } & $\mathrm{F}$ & 11 & $212.20 \pm 13.95$ & $238.90,196.42$ \\
\hline & M & 10 & $223.45 \pm 16.88$ & $259.41,202.34$ \\
\hline \multirow[t]{2}{*}{ Hylobates lar } & $\mathrm{F}$ & 10 & $215.86 \pm 13.51$ & $240.29,199.39$ \\
\hline & M & 7 & $200.80 \pm 13.64$ & $218.11,176.516$ \\
\hline \multirow[t]{2}{*}{ Colobus guereza } & $\mathrm{F}$ & 3 & $148.56 \pm 12.70$ & $159.65,134.70$ \\
\hline & M & 2 & $142.63 \pm 13.42$ & $152.12,133.15$ \\
\hline \multirow[t]{2}{*}{ Nasalis larvatus } & $\mathrm{F}$ & 9 & $188.74 \pm 20.44$ & $217.45,161.69$ \\
\hline & M & 8 & $216.37 \pm 15.57$ & $237.27,189.26$ \\
\hline Piliocolobus badius & $\mathrm{F}$ & 4 & $159.00 \pm 12.13$ & $175.08,147.63$ \\
\hline \multirow{2}{*}{ Rhinopithecus roxellana } & $\mathrm{F}$ & 2 & $126.76 \pm 57.48$ & $167.40,86.12$ \\
\hline & M & 1 & 200.43 & NA \\
\hline \multirow[t]{2}{*}{ Trachypithecus cristatus } & $\mathrm{F}$ & 8 & $152.88 \pm 10.26$ & $166.53,134.22$ \\
\hline & M & 8 & $154.74 \pm 12.52$ & $173.76,133.47$ \\
\hline \multirow[t]{2}{*}{ Pygathrix nemaeus } & $\mathrm{F}$ & 2 & $178.42 \pm 7.97$ & $184.06,172.79$ \\
\hline & M & 5 & $218.27 \pm 15.72$ & $243.47,202.74$ \\
\hline \multirow{2}{*}{ Cercopithecus mitis } & $\mathrm{F}$ & 4 & $120.73 \pm 15.78$ & $142.29,107.86$ \\
\hline & M & 4 & $143.42 \pm 14.18$ & $164.02,131.57$ \\
\hline \multirow[t]{2}{*}{ Erythrocebus patas } & $\mathrm{F}$ & 2 & $131.30 \pm 1.47$ & $132.34,130.26$ \\
\hline & M & 3 & $131.98 \pm 25.74$ & $161.70,117.11$ \\
\hline \multirow[t]{2}{*}{ Macaca fascicularis } & $\mathrm{F}$ & 6 & $113.93 \pm 10.88$ & $128.53,100.00$ \\
\hline & M & 4 & $124.06 \pm 1.02$ & $124.77,122.58$ \\
\hline \multirow[t]{2}{*}{ Macaca nemestrina } & $\mathrm{F}$ & 5 & $156.16 \pm 14.30$ & $173.18,139.24$ \\
\hline & M & 1 & 200.53 & NA \\
\hline \multirow[t]{2}{*}{ Papio cynocephalus } & $\mathrm{F}$ & 7 & $171.36 \pm 19.18$ & $201.86,145.44$ \\
\hline & M & 1 & 196.98 & NA \\
\hline Papio anubis & $\mathrm{F}$ & 5 & $175.28 \pm 30.69$ & $215.16,151.13$ \\
\hline & M & 4 & $210.50 \pm 5.84$ & $218.61,205.45$ \\
\hline Theropithecus gelada & $\mathrm{F}$ & 6 & $156.97 \pm 18.15$ & $177.08,127.42$ \\
\hline & M & 3 & $172.71 \pm 15.94$ & $186.62,155.33$ \\
\hline Ateles belzebuth & $\mathrm{F}$ & 2 & $203.07 \pm 8.91$ & $209.37,196.77$ \\
\hline Ateles fusciceps & $\mathrm{F}$ & 4 & $195.42 \pm 11.97$ & $212.54,184.70$ \\
\hline & M & 2 & $176.85 \pm 3.17$ & $179.10,174.61$ \\
\hline Ateles geoffroyi & $\mathrm{F}$ & 3 & $188.16 \pm 3.35$ & $190.81,184.39$ \\
\hline Ateles paniscus & M & 3 & $210.60 \pm 25.03$ & $239.47,194.94$ \\
\hline Alouatta caraya & $\mathrm{F}$ & 5 & $151.00 \pm 12.12$ & $162.65,137.10$ \\
\hline & M & 5 & $158.75 \pm 4.07$ & $162.26,152.44$ \\
\hline Cebus apella & $\mathrm{F}$ & 6 & $107.06 \pm 12.73$ & $120.55,93.09$ \\
\hline & $M$ & 3 & $112.37 \pm 7.47$ & $119.90,104.96$ \\
\hline
\end{tabular}


Table 4.10 ANOVAs for angular abduction

\begin{tabular}{lrr}
\hline Source & $\mathrm{F}$ & Sig. \\
\hline Locomotor group (suspensory or non-suspensory) & 526.76 & $<0.01$ \\
Data type (in vivo or in silico) & 0.63 & 0.43 \\
Locomotor group * Data type & 115.14 & $<0.01$
\end{tabular}

This comparison excludes Trachypithecus due to the in vivo sample size for this genus. 
Table 4.11 Means by locomotor group and treatment type

\begin{tabular}{llrr}
\hline Locomotor group & Data type & $\begin{array}{r}\text { Mean } \\
\text { Abduction } \\
\text { (degrees) }\end{array}$ & $\begin{array}{r}\text { Abducted knee } \\
\text { position }(\mathrm{mm})\end{array}$ \\
\hline non-suspensory & in silico & 40.41 & 115.36 \\
& in vivo & 25.43 & 115.54 \\
suspensory & in silico & 56.94 & 285.91 \\
& in vivo & 69.67 & 321.25
\end{tabular}

This comparison excludes Trachypithecus due to the in vivo sample size for this genus. Tables 4.8 and 4.11 provide statistical tests for these data. 
Table 4.12 ANOVAs for knee position

\begin{tabular}{lrr}
\hline Source & $\mathrm{F}$ & Sig. \\
\hline Locomotor group (suspensory vs. non-suspensory) & 754.85 & $<0.01$ \\
Data type (in vivo vs. in silico) & 14.09 & $<0.01$ \\
Locomotor group * Data type & 17.57 & $<0.01$
\end{tabular}


Table 4.13 Percent difference between sex-specific means by data type

\begin{tabular}{|c|c|c|c|c|c|c|}
\hline \multirow[b]{2}{*}{ Taxon } & \multirow[b]{2}{*}{ sex } & \multirow{2}{*}{$\begin{array}{c}\mathrm{n} \\
\text { in silico }\end{array}$} & \multirow{2}{*}{$\begin{array}{c}\mathrm{n} \\
\text { in vivo }\end{array}$} & \multicolumn{2}{|c|}{ mean } & \multirow{2}{*}{$\begin{array}{r}\% \text { difference } \\
\text { between } \\
\text { means }\end{array}$} \\
\hline & & & & in silico & in vivo & \\
\hline \multicolumn{7}{|c|}{ Angular abduction (degrees) } \\
\hline \multirow[t]{2}{*}{ Pongo } & $f$ & 13 & 3 & 75.58 & 82.33 & -8.94 \\
\hline & $\mathrm{m}$ & 8 & 3 & 72.82 & 81.00 & -11.23 \\
\hline \multirow[t]{2}{*}{ Gorilla gorilla } & $f$ & 8 & 2 & 50.02 & 62.50 & -24.95 \\
\hline & $\mathrm{m}$ & 10 & 4 & 39.69 & 48.00 & -20.94 \\
\hline \multirow[t]{2}{*}{ Pan } & $f$ & 16 & 13 & 56.98 & 75.38 & -32.30 \\
\hline & $\mathrm{m}$ & 13 & 18 & 54.77 & 70.06 & -27.91 \\
\hline \multirow[t]{2}{*}{ Hylobates } & $f$ & 10 & 1 & 60.16 & 69.00 & -14.69 \\
\hline & $\mathrm{m}$ & 7 & 1 & 55.50 & 69.00 & -24.32 \\
\hline \multirow[t]{2}{*}{ Symphalangus } & $f$ & 11 & 2 & 57.12 & 52.00 & 8.96 \\
\hline & $\mathrm{m}$ & 10 & 2 & 56.45 & 67.50 & -19.57 \\
\hline \multirow[t]{2}{*}{ Colobus } & $f$ & 3 & 1 & 34.16 & 30.00 & 12.18 \\
\hline & $\mathrm{m}$ & 2 & 1 & 29.55 & 26.00 & 12.01 \\
\hline Trachypithecus & $\mathrm{m}$ & 8 & 1 & 47.16 & 41.00 & 13.06 \\
\hline \multirow[t]{2}{*}{ Macaca } & $f$ & 11 & 16 & 47.19 & 25.83 & 45.27 \\
\hline & $\mathrm{m}$ & 5 & 16 & 61.89 & 24.94 & 59.71 \\
\hline \multirow[t]{2}{*}{ Papio } & $f$ & 12 & 3 & 51.52 & 30.67 & 40.48 \\
\hline & $\mathrm{m}$ & 5 & 5 & 37.60 & 18.40 & 51.06 \\
\hline \multirow[t]{2}{*}{ Ateles } & $f$ & 9 & 3 & 53.30 & 62.33 & -16.95 \\
\hline & $\mathrm{m}$ & 5 & 3 & 51.03 & 70.33 & -37.83 \\
\hline \multirow[t]{2}{*}{ Cebus } & $f$ & 6 & 4 & 42.65 & 34.75 & 18.52 \\
\hline & $\mathrm{m}$ & 3 & 2 & 41.18 & 49.00 & -18.99 \\
\hline \multicolumn{7}{|l|}{ Knee position (mm) } \\
\hline \multirow[t]{2}{*}{ Pongo } & $f$ & 10 & 2 & 293.87 & 315.00 & -7.19 \\
\hline & $\mathrm{m}$ & 5 & 2 & 332.15 & 327.50 & 1.40 \\
\hline \multirow[t]{2}{*}{ Gorilla } & $f$ & 8 & 2 & 324.02 & 332.50 & -2.62 \\
\hline & $\mathrm{m}$ & 10 & 4 & 353.31 & 357.50 & -1.19 \\
\hline \multirow[t]{2}{*}{ Pan } & $f$ & 10 & 10 & 301.59 & 346.00 & -14.73 \\
\hline & $\mathrm{m}$ & 8 & 13 & 298.29 & 360.00 & -20.69 \\
\hline \multirow[t]{2}{*}{ Symphalangus } & $f$ & 11 & 1 & 212.2 & 255.00 & -20.17 \\
\hline & $\mathrm{m}$ & 10 & 1 & 223.95 & 275.00 & -22.80 \\
\hline Colobus & $f$ & 3 & 1 & 148.56 & 145.00 & 2.40 \\
\hline \multirow[t]{2}{*}{ Macaca } & $f$ & 6 & 8 & 113.93 & 106.56 & 6.47 \\
\hline & $\mathrm{m}$ & 4 & 8 & 124.06 & 120.63 & 2.76 \\
\hline \multirow[t]{2}{*}{ Cebus } & $f$ & 6 & 4 & 107.06 & 116.25 & -8.58 \\
\hline & $\mathrm{m}$ & 3 & 2 & 112.37 & 135.00 & -20.14 \\
\hline
\end{tabular}

Percent difference was calculated here as ((in silico mean-in vivo mean)/in silico)^100. 
Table 4.14 Tukey's HSD comparisons between data types (in vivo vs. in silico) for angle of abduction

\begin{tabular}{lr}
\hline & in vivo vs. in silico p-value \\
\hline Pongo & 0.74 \\
G. gorilla & 0.43 \\
Pan & $<0.01$ \\
Hylobates & 0.21 \\
Symphalangus & 1.00 \\
Colobus & 1.00 \\
Macaca & $<0.01$ \\
Papio & $<0.01$ \\
Ateles & $<0.01$ \\
Cebus & 1.00
\end{tabular}

Significance was assessed at $p \leq 0.05$. 
Table 4.15 Tukey's HSD comparisons between data types (in vivo vs. in silico) for abducted knee position

\begin{tabular}{lr}
\hline & in vivo vs. in silico p-value \\
\hline$P$. pygmaeus & 0.99 \\
G. gorilla & 1.00 \\
P. troglodytes & $<0.01$ \\
S. syndactylus & 0.10 \\
M. fascicularis & 1.00 \\
C. apella & 0.99
\end{tabular}

Significance was assessed at $p \leq 0.05$. 


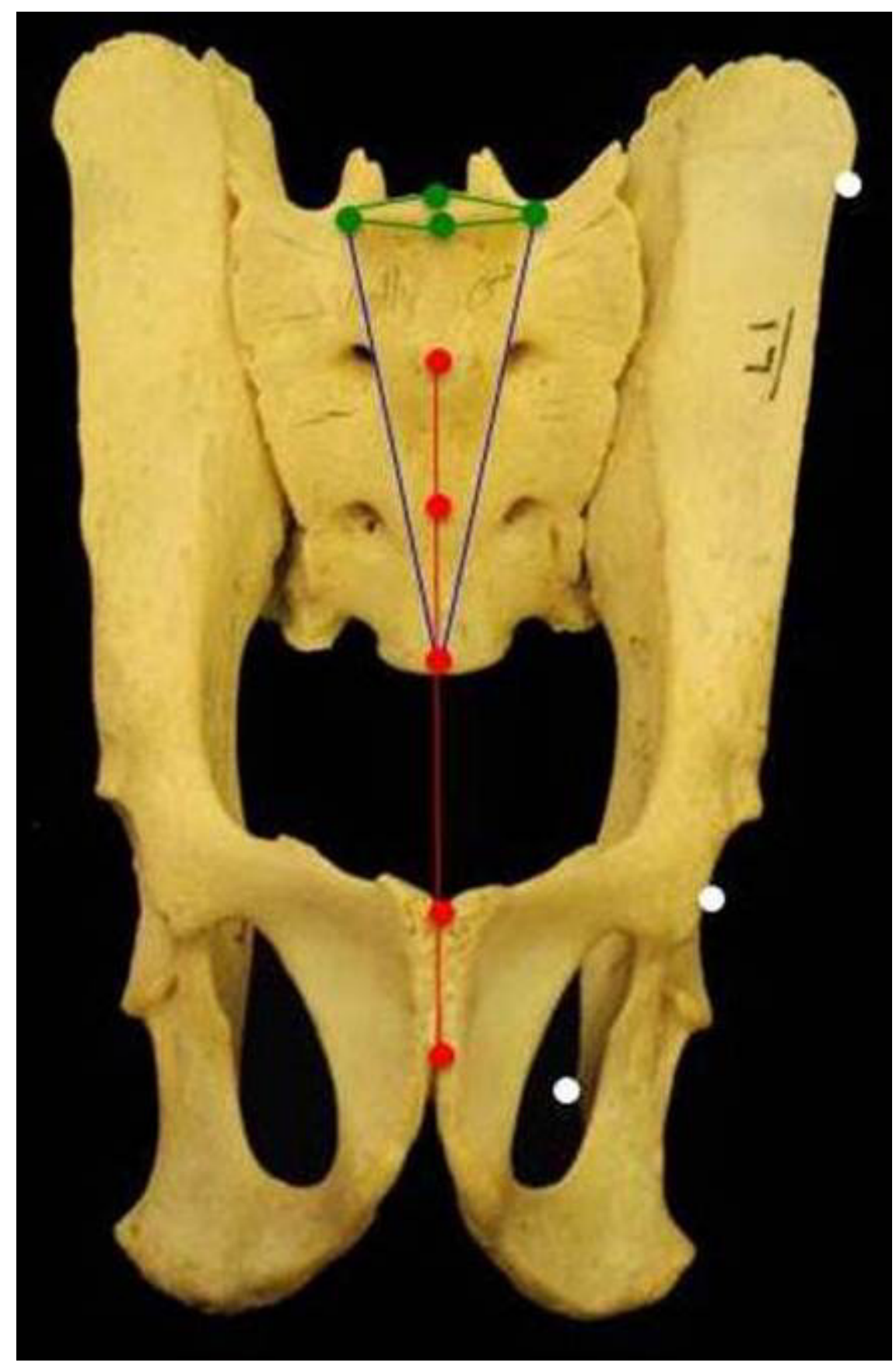

Figure 4.1 Microscribe landmarks are superimposed on a pelvis

PIIS (landmark 1) and the center of the acetabular fossa (landmark 4) are not visible due to the orientation of the pelvis. 

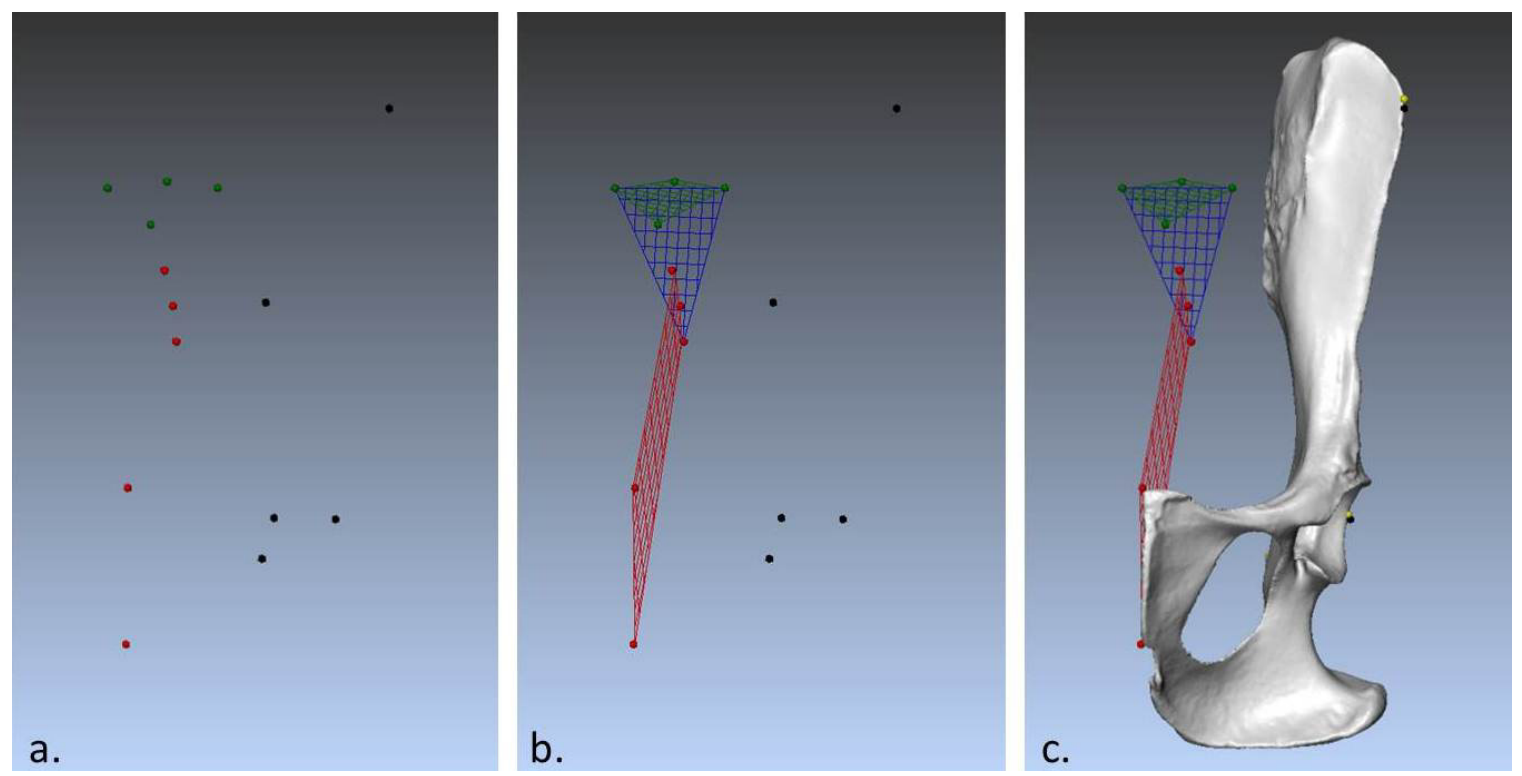

Figure 4.2 Pelvis polygonal model alignment to landmark data is shown for Papio cynocephalus (OM7276) in the IMInspect module in PolyWorks

Points are created for the landmark coordinate data imported into IMInspect (a). Planes can then be established to the points (b). Corresponding points anchored to the pelvis are then aligned to the points for the ASIS, PIIS, center of the acetabular fossa, dorsal acetabular border, and ischial spine. 

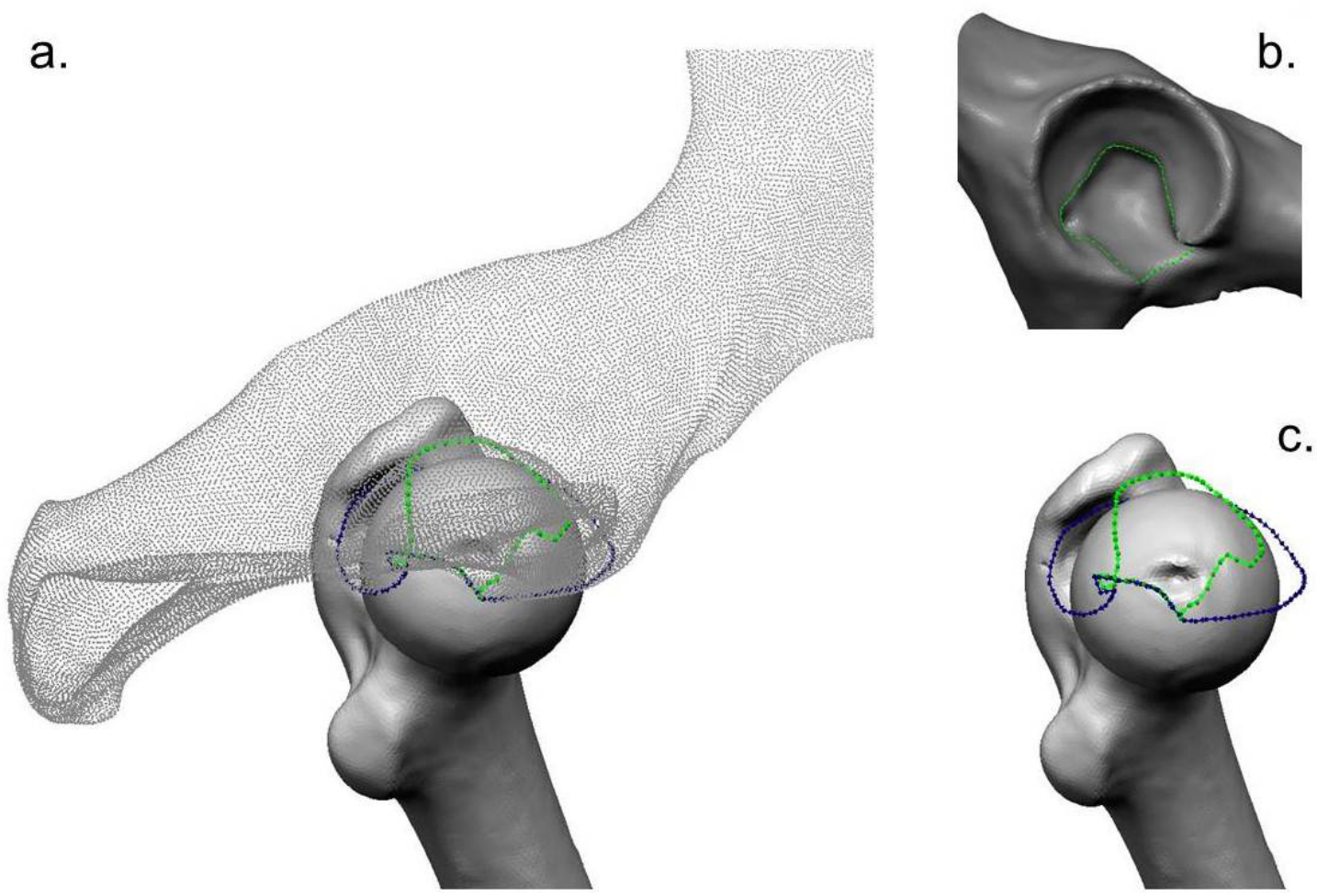

Figure 4.3 The fovea capitis is confined to the boundaries of the acetabular fossa An articulated model of Pan troglodytes (CMNH B1708 ${ }^{\lambda}$ ), with transparent pelvis is shown (a). The acetabular fossa is outlined in green on the acetabulum (b) and superimposed over the corresponding femoral head (c). 


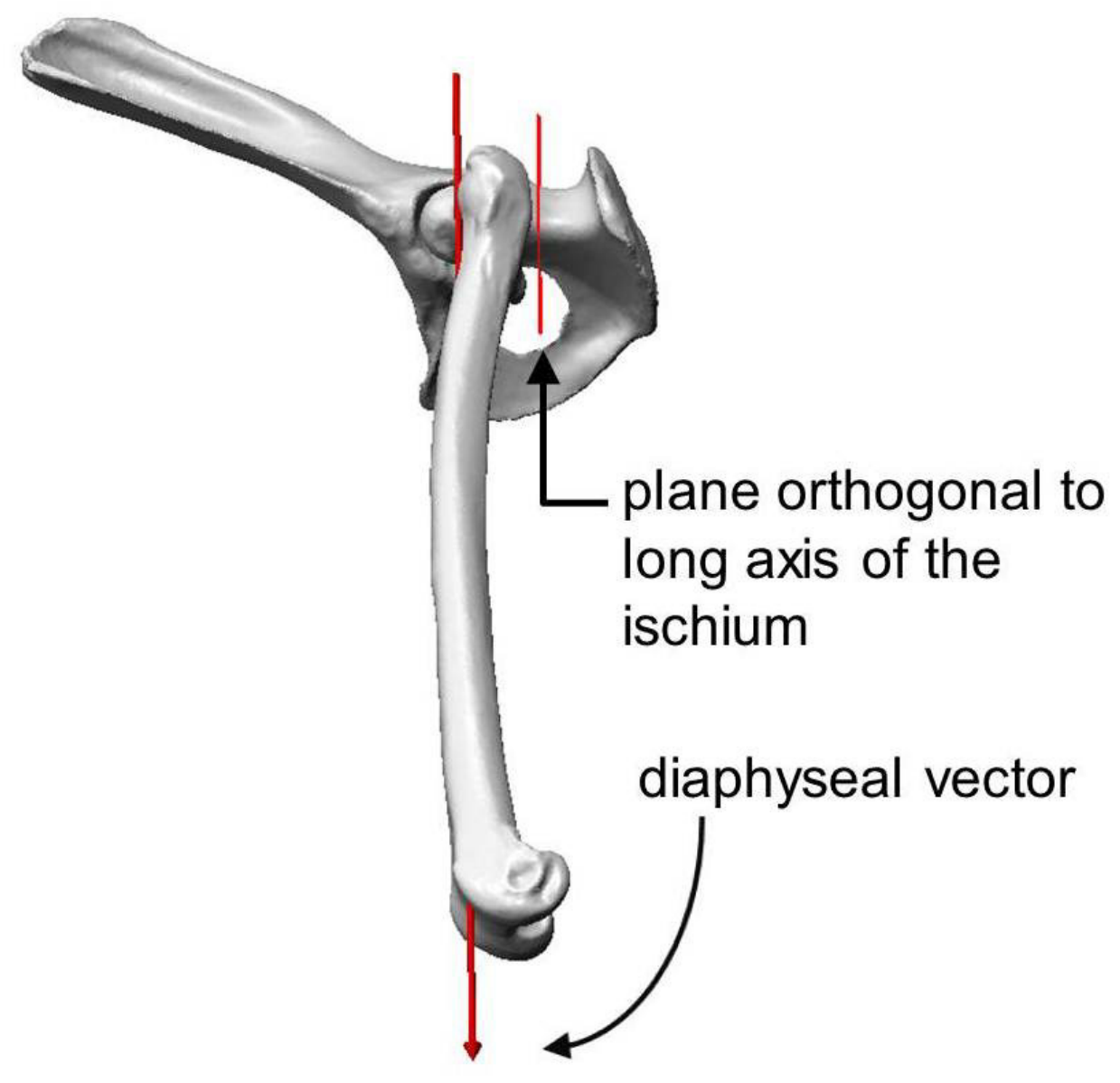

Figure 4.4 The femoral diaphysis is oriented parallel to an orthogonal plane to the long axis of the ischium 

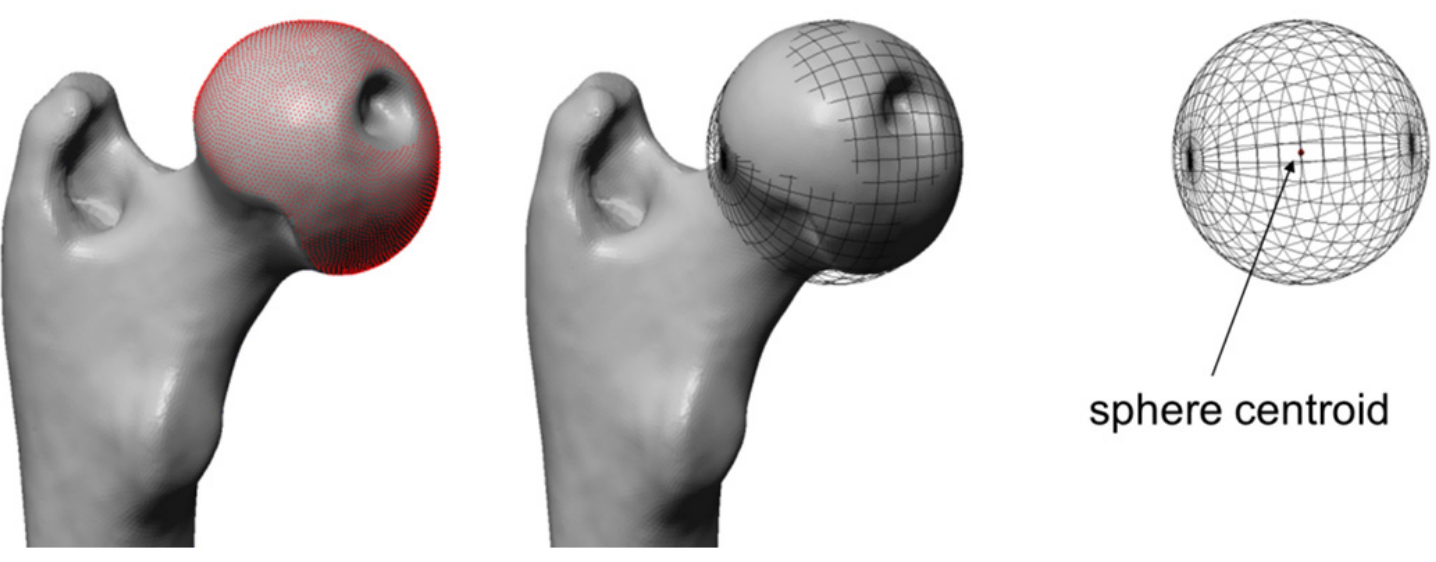

Figure 4.5 Femoral head least squares sphere-fitting and centroid creation

The femoral head subchondral surface is selected, a least squares best-fit sphere is fit to the selected data points, and a sphere centroid is identified. Symphalangus syndactylus (ZSM 1905.60 \%) is shown. 


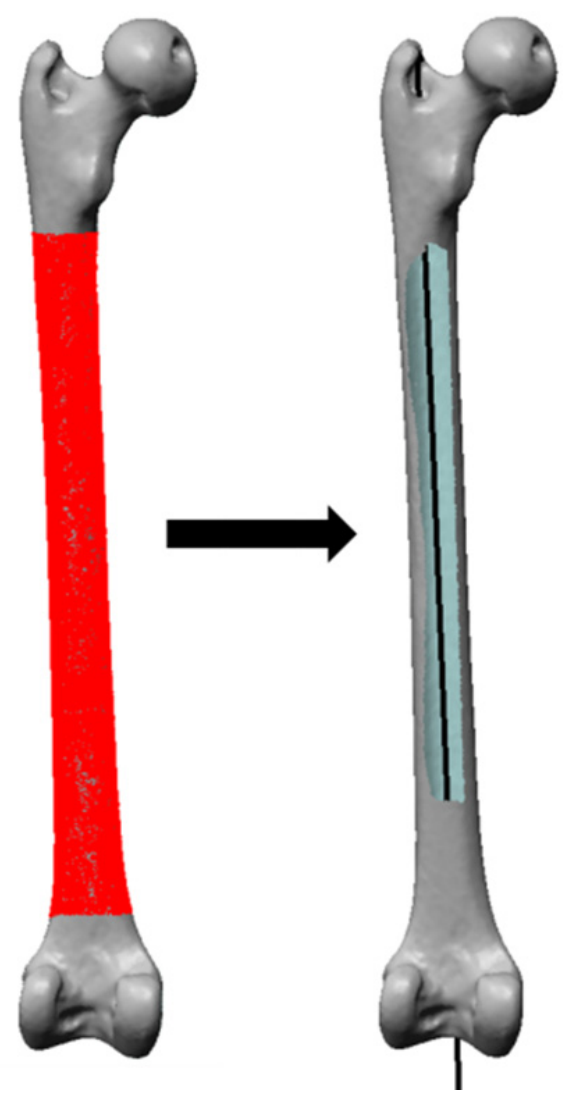

Figure 4.6 Femoral diaphysis least squares vector-fitting

The femoral diaphysis was selected beginning just inferior to the lesser trochanter and superior to the initial flaring for the femoral condyles. A least squares best-fit vector is then fit to the selected data points. The femur on the right has a section of the diaphysis removed to show the vector running inside the polygonal model. Symphalangus syndactylus (ZSM 1905.60 o) is shown. 
a.

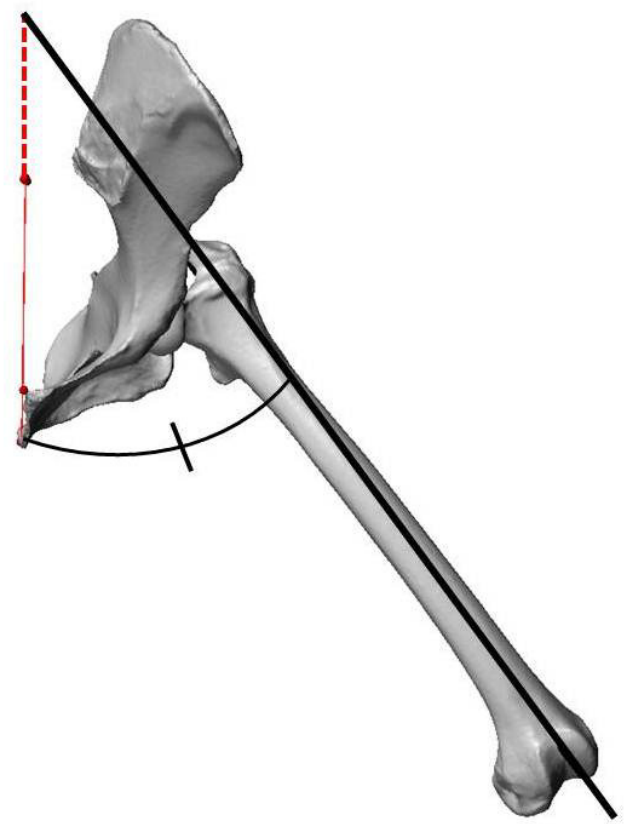

b.

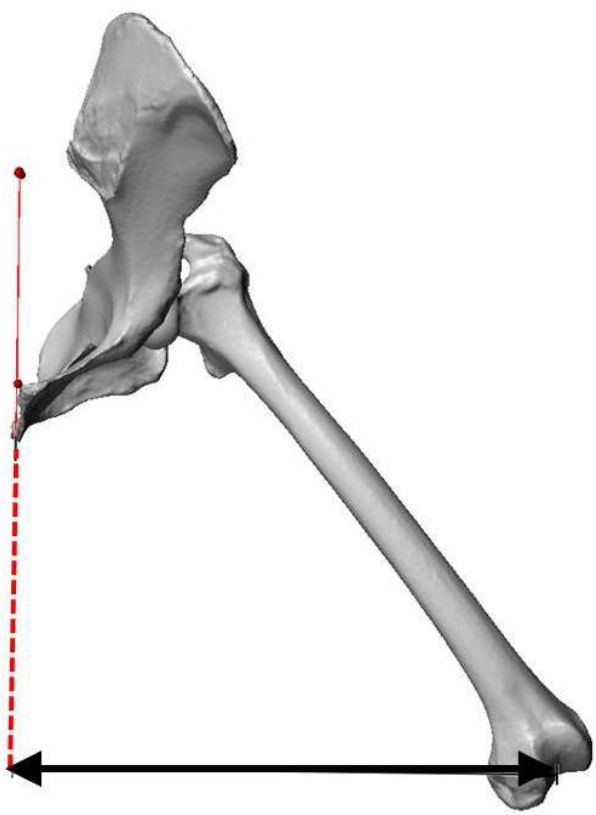

Figure 4.7 Measures of abduction (degrees) and abducted knee position ( $\mathrm{mm}$ ) relative to the midline

(a) Angular abduction and (b) the span at the knee, a proxy for the hindlimb spatial envelope. The red dashed line indicates where the midline plane is when extended from its vertices. Papio anubis (NMNH 236976 +) shown. 


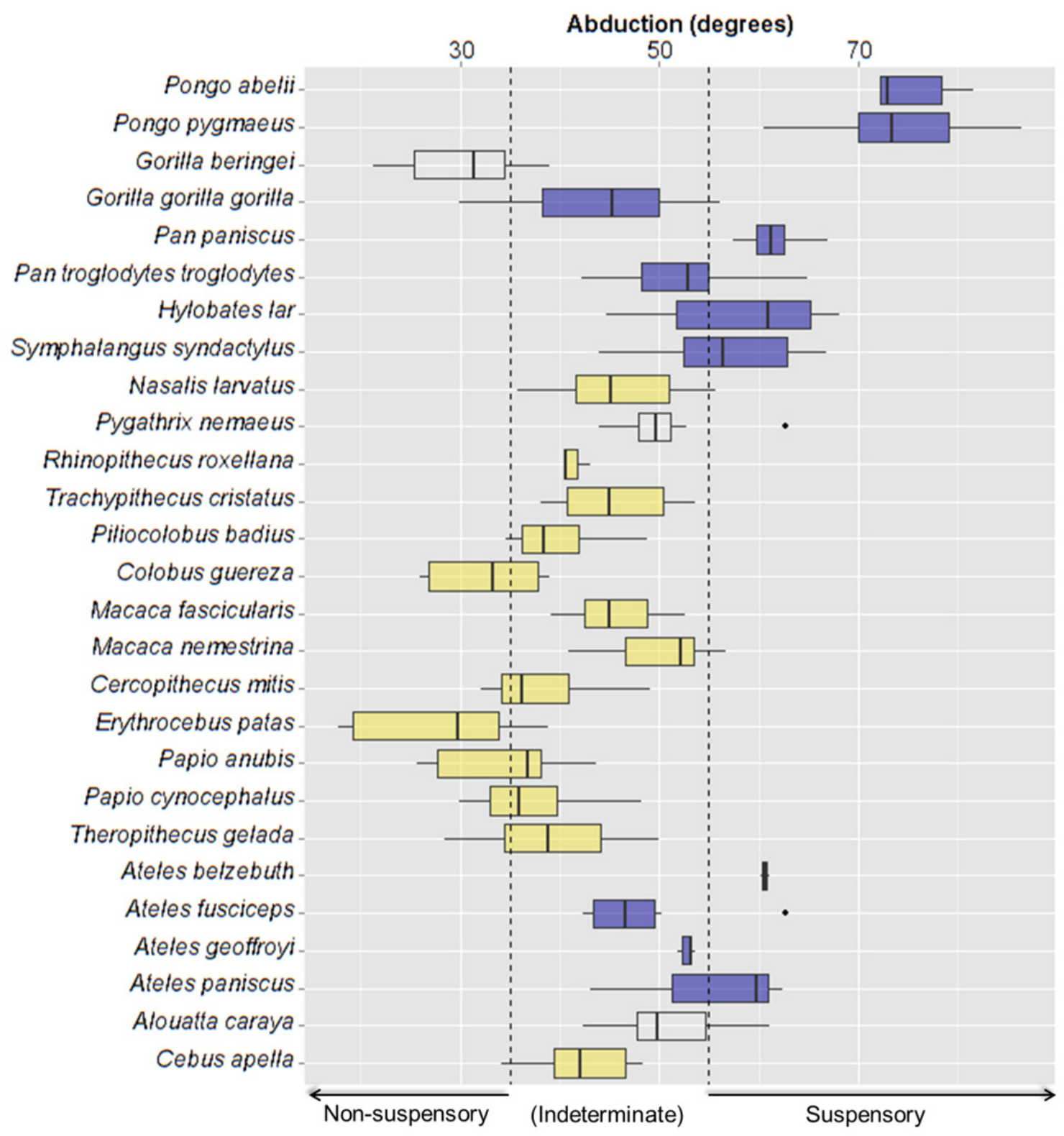

Figure 4.8 In silico measures of abduction (degrees)

Suspensory taxa are indicated by the darker blue shaded boxplots and non-suspensory taxa are indicated by yellow boxplots. No shading indicates an unassigned locomotor category in fossils and certain extant taxa (G. beringei, A. caraya, P. nemaeus). Suspensory and non-suspensory taxa are significantly different in abducted knee position (one-way ANOVA, $p<0.01$ ), but there is substantial overlap between locomotor groups between $35-55^{\circ}$ abduction. 


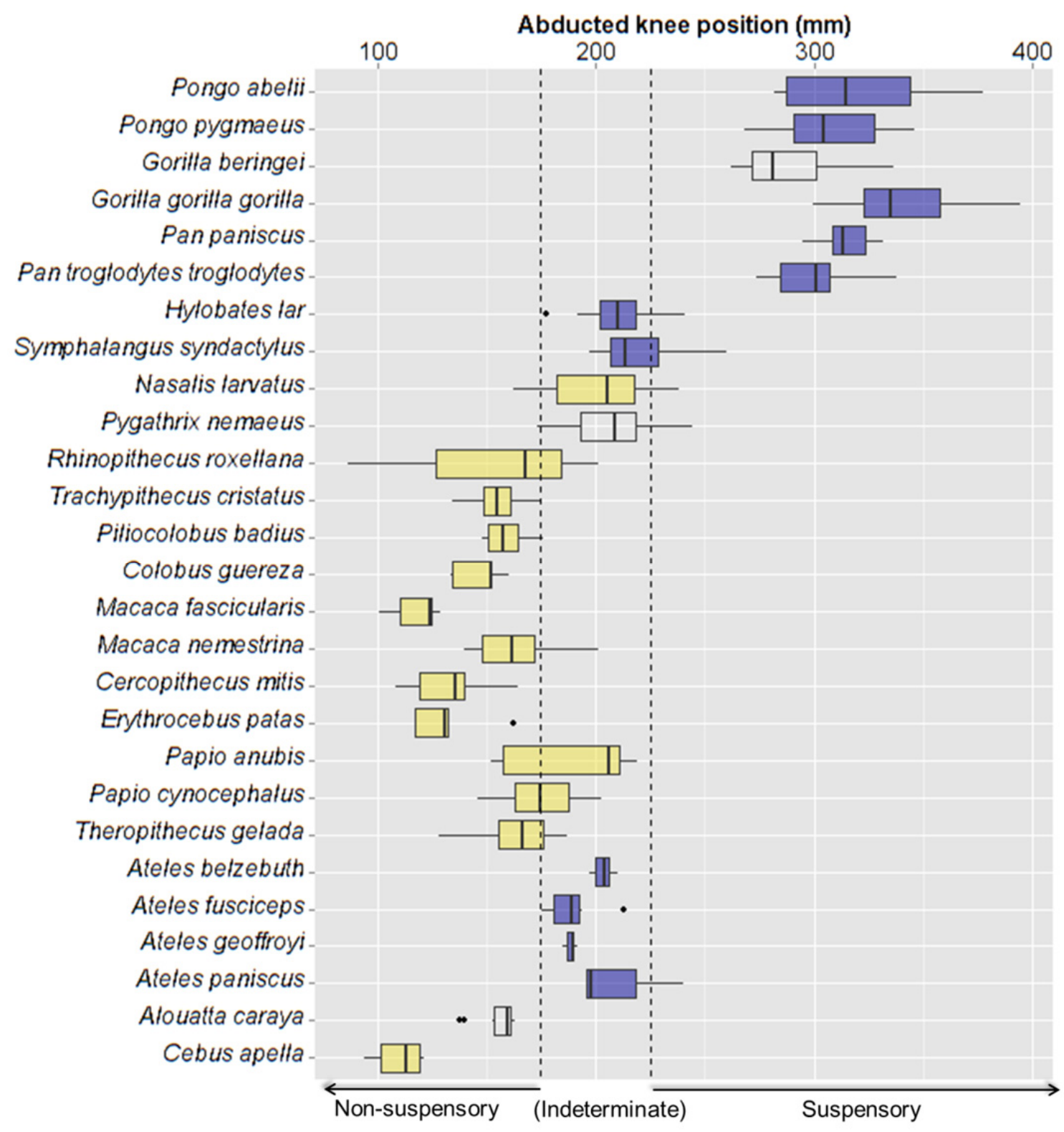

Figure 4.9 In silico measures of abducted knee position $(\mathrm{mm})$

Suspensory taxa are indicated by the darker blue shaded boxplots and non-suspensory taxa are indicated by yellow boxplots. No shading indicates an unassigned locomotor category in fossils and certain extant taxa (G. beringei, A. caraya, $P$. nemaeus).

Although suspensory and non-suspensory taxa are significantly different in abducted knee position relative to the midline (one-way ANOVA, $p<0.01$ ), there is some overlap between locomotor groups in between $175-225 \mathrm{~mm}$. 


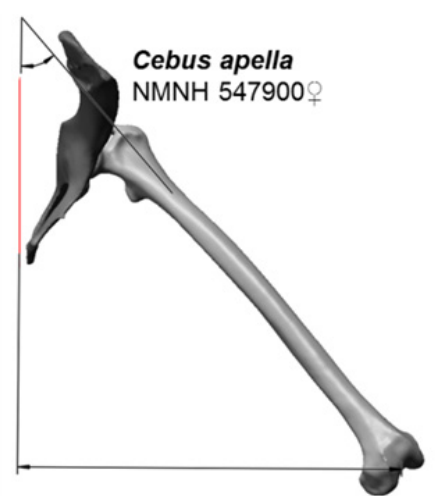

C. apella mean:

$42^{\circ}$ abduction

$107 \mathrm{~mm}$ abducted knee position

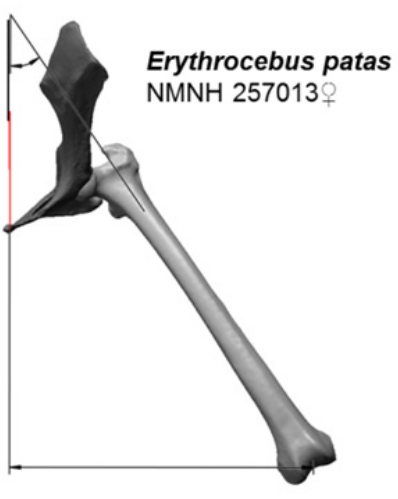

E. patas $\&$ mean:

$36^{\circ}$ mean abduction

$131 \mathrm{~mm}$ abducted knee position

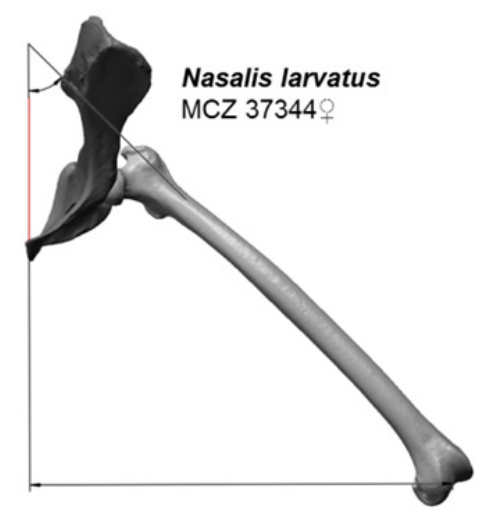

$N$. larvatus $\&$ mean:

$45^{\circ}$ abduction

$189 \mathrm{~mm}$ abducted knee position

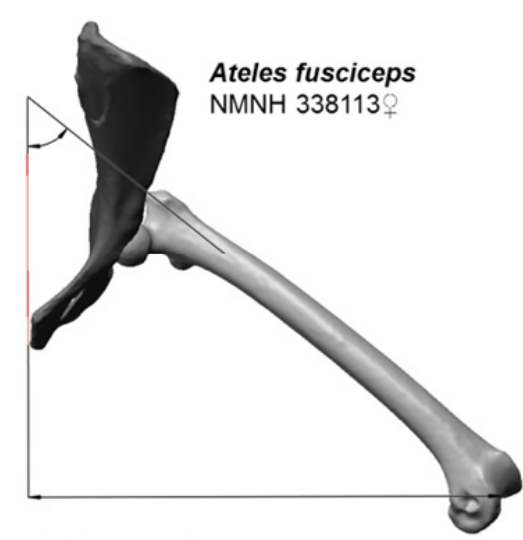

A. fusciceps mean:

$50^{\circ}$ abduction

$195 \mathrm{~mm}$ abducted knee position

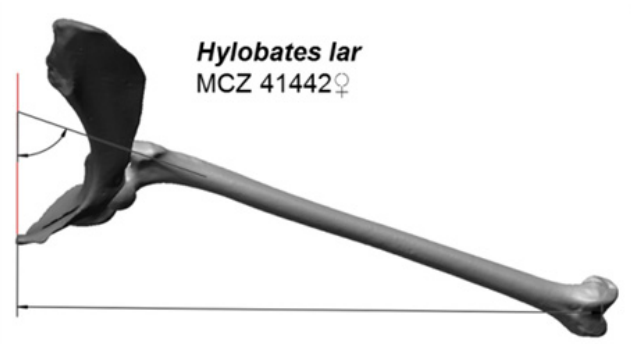

H. lar $q$ mean:

$60^{\circ}$ abduction

$216 \mathrm{~mm}$ abducted knee position

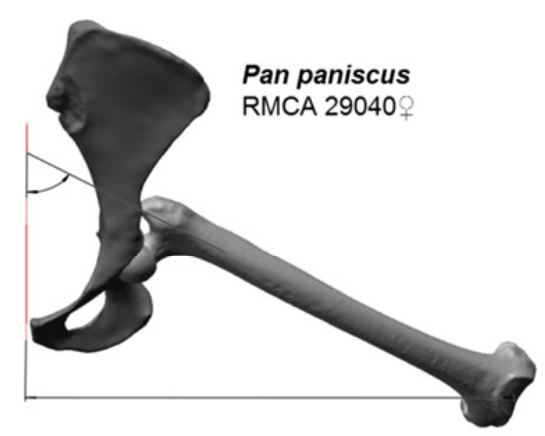

$P$. paniscus + mean:

$60^{\circ}$ abduction

$318 \mathrm{~mm}$ mean abducted knee position

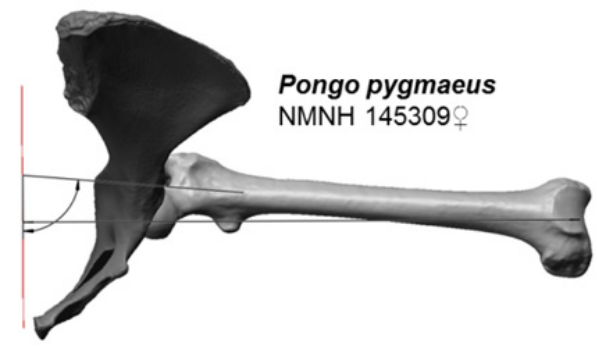

$P$. pygmaeus + mean:

$76^{\circ}$ abduction

$294 \mathrm{~mm}$ mean abducted knee position

Figure 4.10 Visual comparison of in silico measures of abduction (degrees) and abducted knee position $(\mathrm{mm})$ in a subset of female anthropoids

Specimen numbers are provided for the individuals figured. Different taxa are not shown to the same scale. 


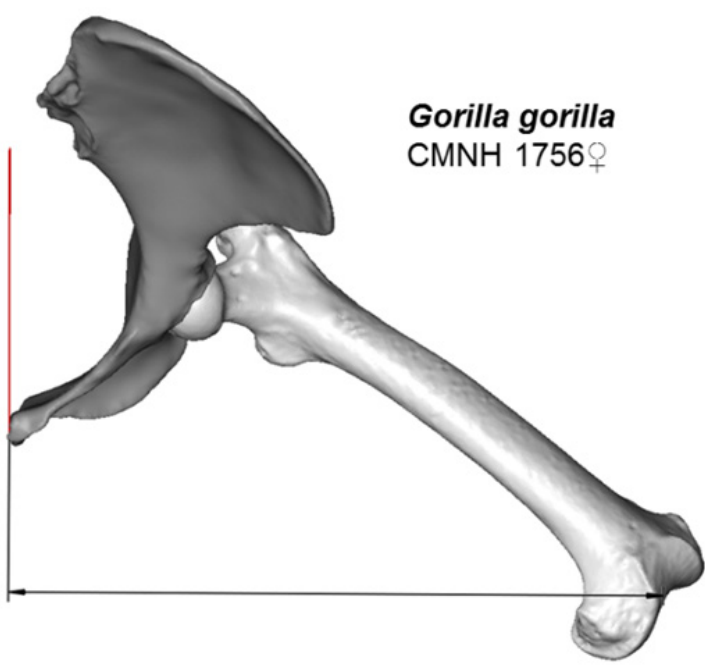

G. gorilla $\odot$ mean:

$50^{\circ}$ abduction

$324 \mathrm{~mm}$ abducted knee position

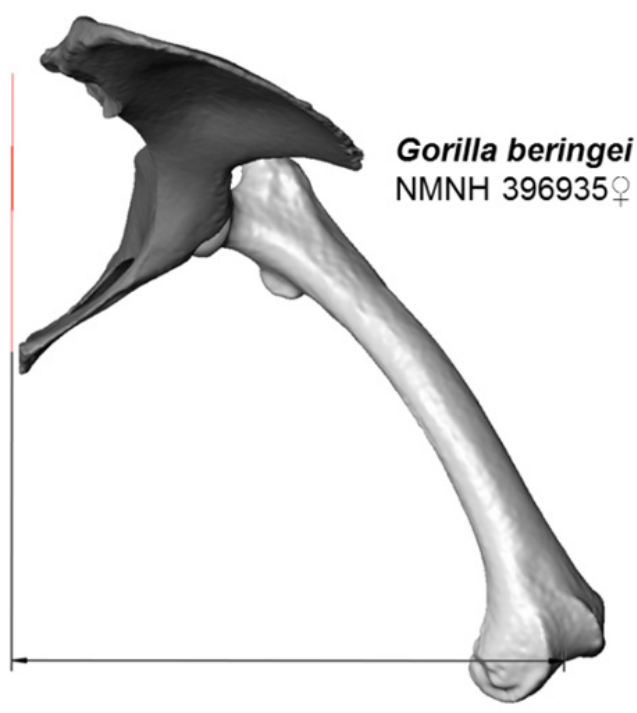

G. beringei + mean:

$34^{\circ}$ abduction

$274 \mathrm{~mm}$ abducted knee position

Figure 4.11 Visual comparison of in silico measures of abduction (degrees) and abducted knee position ( $\mathrm{mm}$ ) in female Gorilla gorilla and Gorilla beringei

Specimen numbers are provided for the individuals figured. Individuals are not shown to the same scale. 

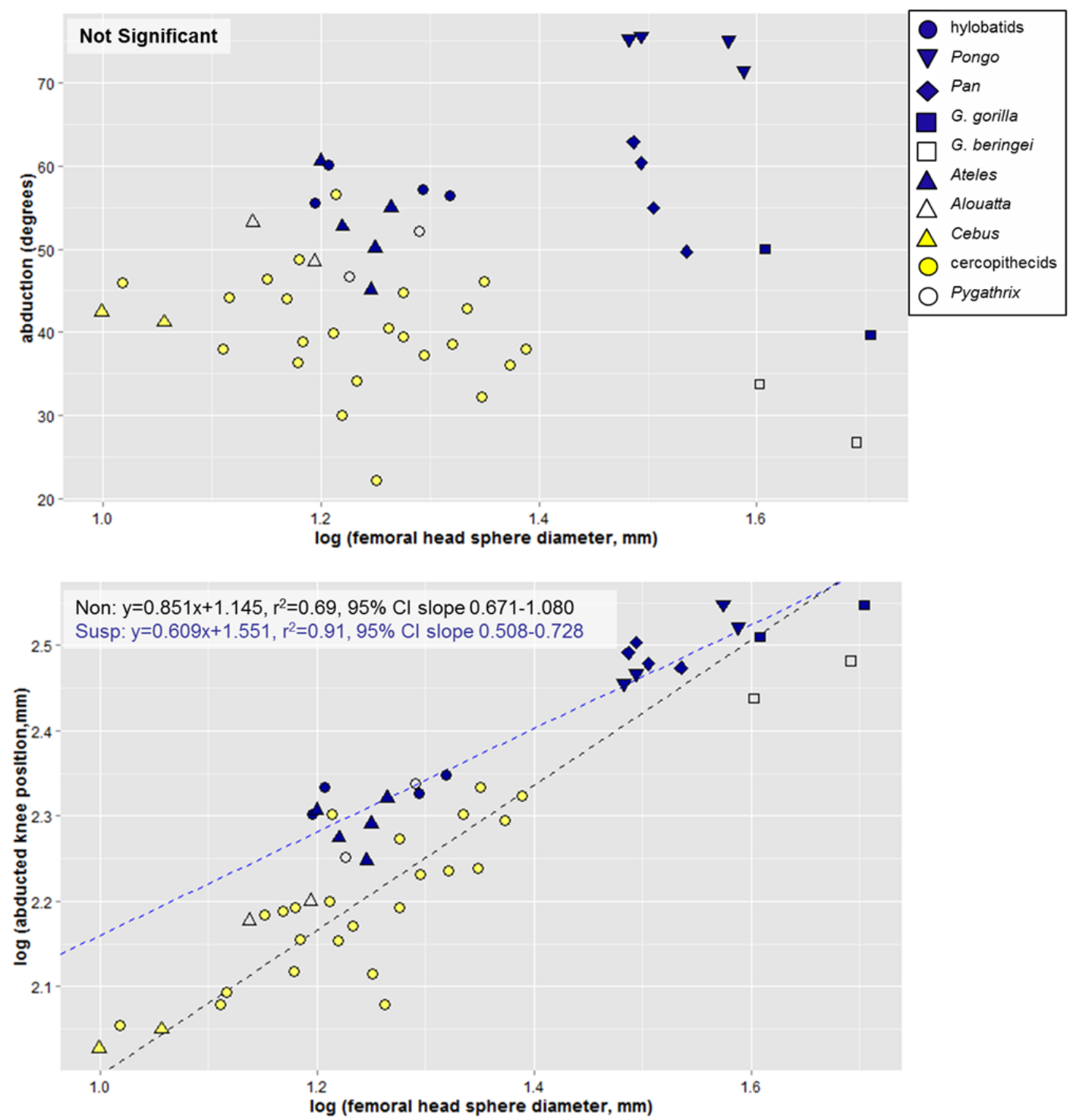

Figure 4.12 In silico measures of abduction (degrees) and abducted knee position $(\mathrm{mm})$ on the femoral head sphere diameter using standard major axis regression The relationship between abduction and the body size surrogate is not significant across anthropoids or within locomotor groups. The relationship between body size and abducted knee position is significant, with significant differences in slope $(p=0.025)$ detected between locomotor groups. Regressions for suspensory (blue dashed line) and non-suspensory taxa (black dashed line) are indicated by dashed lines. Gorilla beringei, Alouatta caraya, and Pygathrix nemaeus were not included in the regression calculations but are also plotted. NS=non-significant. 


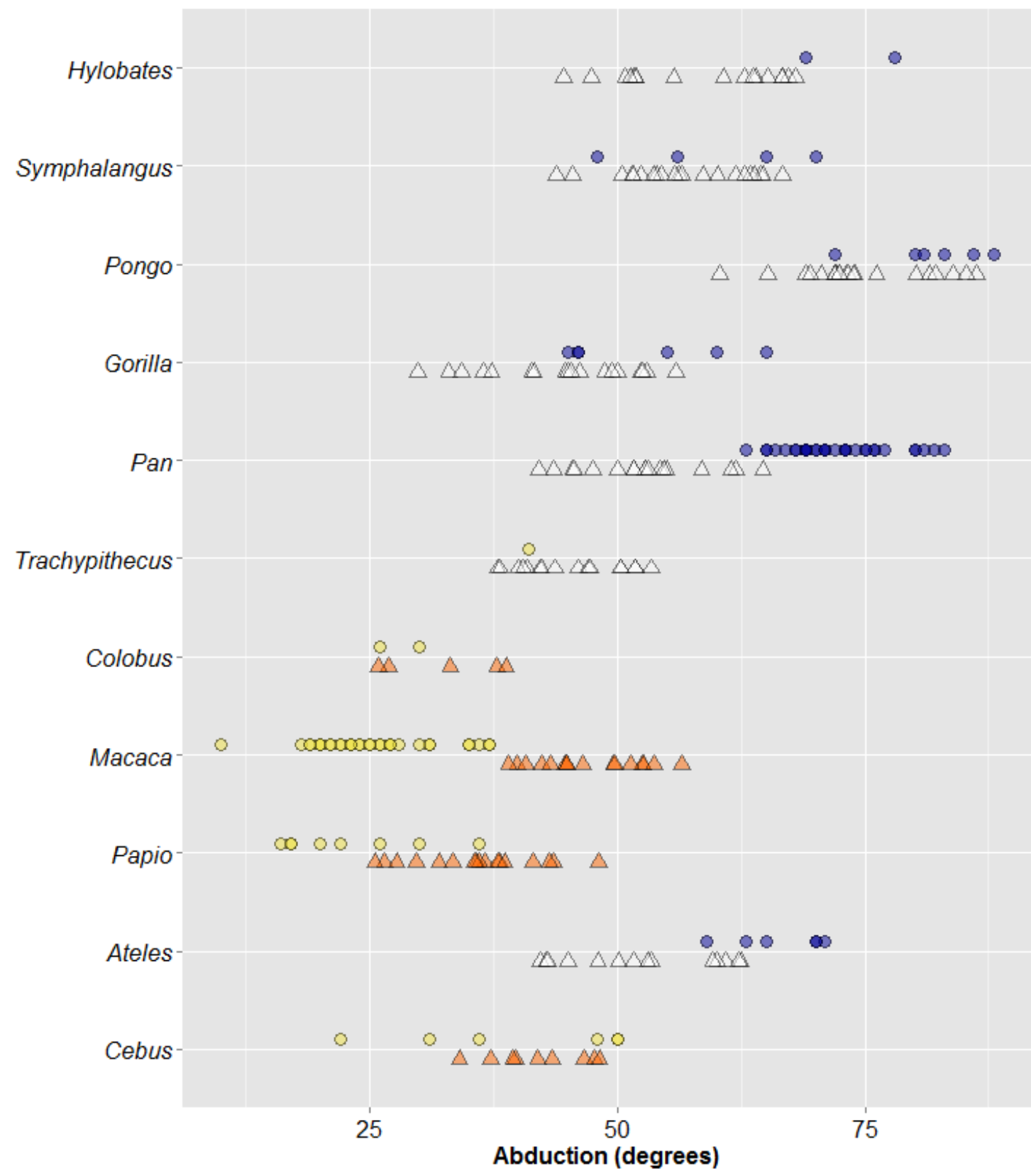

Figure 4.13 Comparison of in vivo and in silico data points for angular abduction by locomotor group

Triangles represent in silico data, with circles positioned above indicating in vivo data points. Data have been color coded by data type and locomotor group to maximize visualization of the distribution. Data (both in vivo and in silico) for male Trachypithecus are shown in this figure but Trachypithecus was not included in the two-way ANOVA (Table 4.10) and descriptive statistics (Table 4.9) due to the small in vivo sample $(n=1)$. 


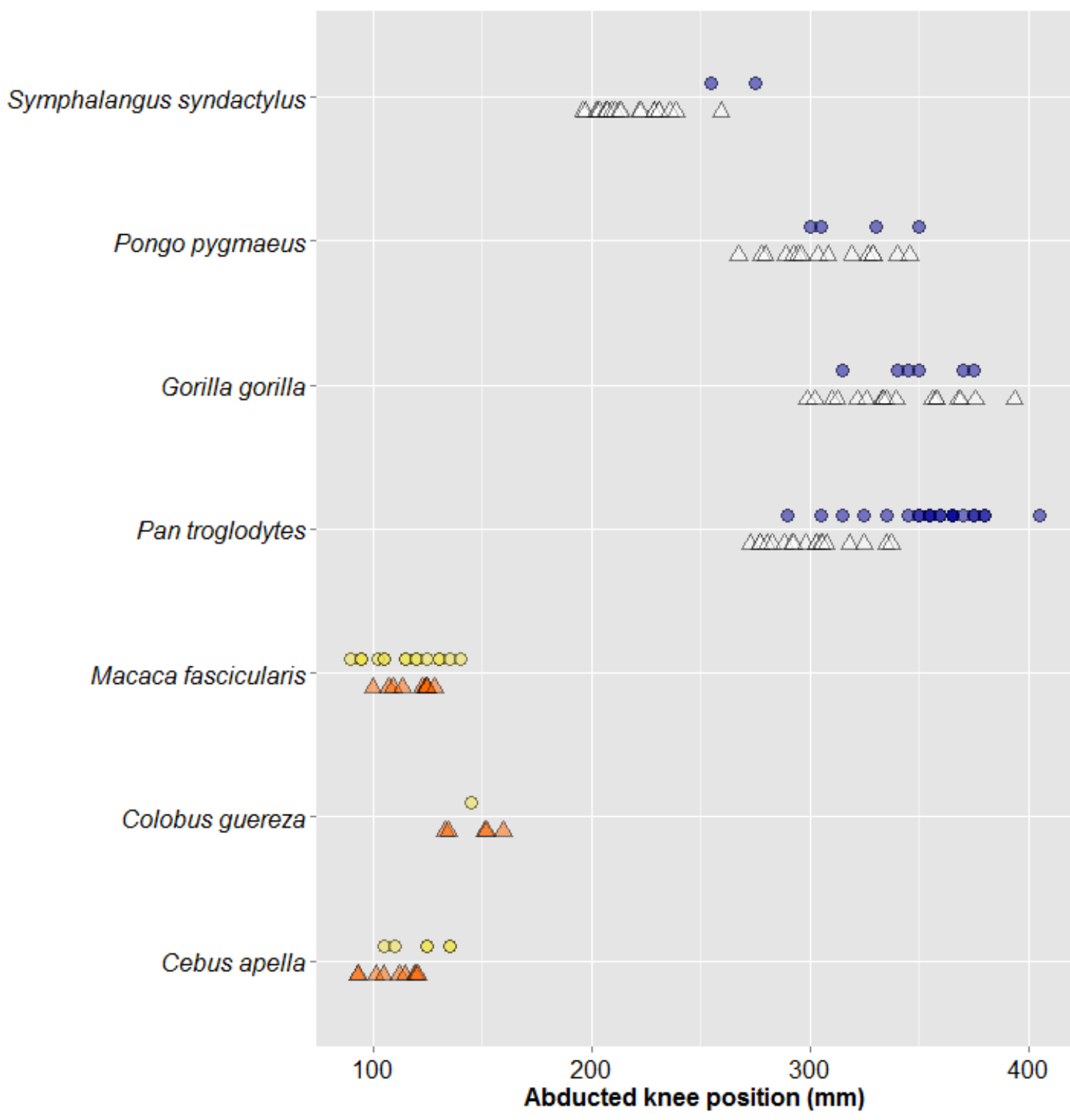

Figure 4.14 Comparison of in vivo and in silico data points for abducted knee position by locomotor group

Triangles represent in silico data, with circles positioned above indicating in vivo data points. Data have been color coded by data type and locomotor group to maximize visualization of the distribution. In vivo data for knee position were adjusted by dividing the gross span at the knee in half, which adjusts the values to be comparable to a midline plane. 

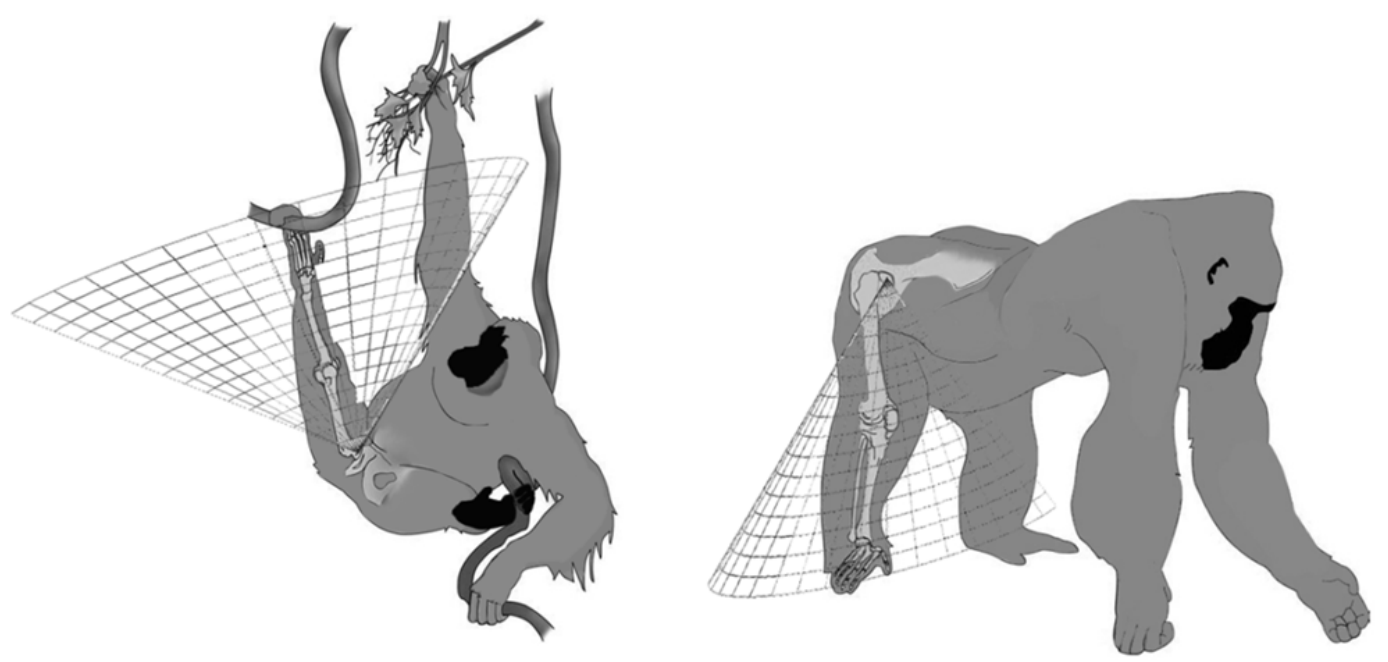

Figure 4.15 Hypothetical examples of the hindlimb spatial envelope

The hindlimb spatial envelope is determined by, but not limited to, body size, hindlimb and pelvic morphology, as well as range of motion allowed at the hindlimb joints.

Orangutans and gorillas have the same abducted knee position (distance from the midline), but the greater joint mobility in orangutans allows them to abduct their hindlimb laterally to the body rather than tucked under it, increasing the range of possible foot postures. Figure modified from Zihlman et al. (2011). 
CHAPTER 5: RECONSTRUCTING HIP JOINT FUNCTION IN FOSSIL CATARRHINES

The goal of this chapter is to use the validated digital modeling approach developed in Chapter 4 to estimate hip abduction ability in fossil catarrhines. In doing so, this chapter will address the overarching goal of this dissertation, which is to test whether suspensory behaviors can be inferred in fossil nonhuman hominoids based on range of hip abduction modeled in silico. Taxa chosen for this analysis are fossil hominoids Rudapithecus hungaricus and Proconsul nyanzae, and fossil cercopithecoids Paracolobus chemeroni, Paracolobus mutiwa, and Theropithecus oswaldi.

The most critical taxon for this study is Rudapithecus hungaricus, which has been interpreted to be a highly suspensory Miocene hominoid. Because Rudapithecus hungaricus preserves a relatively complete femur (RUD 184) and pelvic innominate (undescribed) from the same individual, it provides an opportunity to test whether adaptations for suspensory behaviors can be inferred in fossil nonhuman hominoids based on range of hip abduction. If hip abduction in Rudapithecus is found to be exclusively within the range of extant suspensory species, this will provide corroborative evidence that at least one late Miocene crown hominoid used suspensory behaviors.

Although early Miocene hominoid Proconsul nyanzae (KNM-MW 13142) is one of the most well-studied fossil hominoids, hip morphology in $P$. nyanzae has been highly influential in the locomotor reconstruction for this taxon and all other early hominoids. Proconsul nyanzae displays many postcranial features consistent with arboreal quadrupedalism but has a hip joint that appears to confer high levels of mobility, leading to interpretations of clambering above-branch behaviors (see discussion and references 
below). A re-examination of hip abduction ability in $P$. nyanzae will clarify whether the hindlimb postures in this species are consistent with its locomotor reconstruction or whether the abduction ability is consistent with generalized arboreal quadrupedalism.

In addition, abduction ability is estimated in three large fossil cercopithecoids. These fossil monkeys provide an opportunity to test whether cercopithecoids with a large body size also display more limited hip abduction abilities than suspensory taxa. In doing so, this will also allow us to test the published locomotor reconstructions for these fossil cercopithecoids. The locomotor reconstructions for these large fossil cercopithecoids typically emphasize a significant reliance on terrestrial behaviors, which contrasts with the locomotor reconstructions of Rudapithecus hungaricus, Proconsul nyanzae, and the majority of extant anthropoids.

\section{Fossil taxa and hypothesized locomotor reconstructions}

The locomotor reconstruction for late Miocene Rudapithecus hungaricus (10 Ma, Rudabánya, Hungary) involves a significant reliance on suspensory behaviors (Morbeck 1983; Begun 1992; 1993; Kivell and Begun 2009; Begun and Kordos 2011; Begun et al. 2012). Specifically, Rudapithecus hungaricus has been described as a "generalized suspensory arboreal quadruped" (Begun 1993, p. 373) based on forelimb morphology (Begun 1993; Kivell and Begun 2009; Begun and Kordos 2011; Begun et al. 2012). The distal humerus is described as promoting high elbow mobility (Morbeck 1983), and the scaphoid and capitate are qualitatively similar to Pongo and suspensory taxa (Kivell and Begun 2009). Manual proximal phalanges from Rudabánya also have features found in suspensory anthropoids, including a ventral median groove, well-developed flexor sheath ridges, highly curved shafts, and large round distal articular surfaces (Begun 1988; 1993). The manual proximal phalanges are described as most similar to 
Symphalangus, although hylobatids tend to display higher curvature and more defined flexor ridges than is observed in Rudapithecus (Begun 1988). Little has been published about the Rudabánya pelvic innominate (Ward et al. 2008; Ward et al. 2010; Ward 2013) and femora (Begun and Kordos 2011; Begun et al. 2012), although they are described as having morphology similar to suspensory taxa (see also Chapter 2).

The only other Miocene ape with a relatively complete, undistorted pelvis and femur is Proconsul nyanzae (KNM-MW 13142) from Mfangano Island, Kenya. Early Miocene Proconsul ( $18 \mathrm{Ma}$ ) has primitive torso morphology (Ward 1991; 1993; Ward et al. 1993) and lacked a tail (Ward et al. 1991; Nakatsukasa et al. 2004), but had long and powerful manual and pedal phalanges (Begun 1993; Begun et al. 1994) (see Chapter 2 for review). At odds with most aspects of the locomotor reconstruction is the hip joint, which has features thought to increase range of motion at the hip, including a shallow acetabulum, a wide acetabular notch, a spherical femoral head with even articular surface, and a high neck-shaft angle (Ward 1991; 1993; Ward et al. 1993). The combination of a cercopithecoid-like torso, mobile hip joint, and powerful grasping hands and feet has led to a reconstruction as a clambering quadruped using deliberate, cautious arboreal behaviors (Le Gros Clark and Leakey 1951; McHenry and Corruccini 1983; Walker and Pickford 1983; Beard et al. 1986; Ward 1993; Ward et al. 1993; Kelley 1997; Ward 2007). Early Miocene Proconsul and late Miocene Rudapithecus currently represent the extremes for primitive and derived locomotor reconstructions in Miocene hominoids.

Given that a relatively large femoral head might potentially increase the range of hip excursions (Ruff 1988), it is possible that Proconsul might have a high range of abduction in silico because it had a large femoral head. Because there are no extant 
cercopithecoids that are as large as the great apes, incorporating large-bodied fossil cercopithecoids (27-58 kg, Table 5.1) controls for the possibility that abduction ability in extant non-suspensory species is related to their size rather than locomotor behaviors. Indeed, fossil Theropithecus and Paracolobus have been included as a comparative outgroup in past morphological analyses of Proconsul because Proconsul nyanzae (KNM-MW 13142) body mass estimates fall outside the range of extant monkeys (Ward 1991; 1993). Pelves and femora are preserved for large-bodied Plio-Pleistocene fossil cercopithecoid specimens of Theropithecus oswaldi (Olduvai-MCKII and KNM-ER 866), Paracolobus chemeroni (KNM-BC 3), and Paracolobus mutiwa (KNM-WT 16827). Theropithecus oswaldi, Paracolobus chemeroni, and Paracolobus mutiwa are all reconstructed as quadrupedal with little or no use of suspension, but perhaps using some arboreal behaviors (Table 5.1). Moreover, these large fossil cercopithecoids are hypothesized to use at least some terrestrial behaviors (see discussions below) since it can be difficult for large-bodied animals without suspensory adaptations to move in the canopy.

Theropithecus oswaldi is hypothesized to be a predominantly terrestrial cercopithecine associated with open habitats (Eck 1987; Krentz 1993; Elton 2002). This species has multiple features of the postcrania that indicate terrestrial quadrupedalism, including a dorsally-flexed olecranon process, projecting greater trochanter and tubercles of the humerus, a low neck-shaft angle of the femur, and a short femoral neck (Krentz 1993). Most of the forelimb and hindlimb anatomy is interpreted as aiding powerful protraction-retraction movements of the limbs, suggesting a highly terrestrial and stereotyped locomotor profile (Krentz 1993). Theropithecus oswaldi is known from sites throughout Africa and southern Europe, and is found with high frequency beginning 
at the end of the Pliocene (Jolly 1972; Gibert et al. 1995; Elton 2002; Frost and Delson 2002; Rook et al. 2004). This species is the most widely-represented monkey in the late Pliocene-early Pleistocene fossil record and is usually considered the most terrestrial of the fossil cercopithecoids.

Paracolobus chemeroni is one of the better sampled colobines in East Africa but is primarily known from the KNM-BC 3 skeleton from Baringo, Kenya (Leakey 1969; Birchette 1982; Leakey 1982). Paracolobus chemeroni has been described as a "Iocomotory chimera" because it displays postcranial features common to both terrestrial and arboreal cercopithecines (Hlusko 2007, p. 73). The locomotor reconstruction for Paracolobus chemeroni includes both arboreal and terrestrial behaviors to accommodate the combination of terrestrially-adapted and arboreally-adapted morphologies (Birchette 1982; Leakey 1982; Ting 2001).

The closely related species Paracolobus mutiwa ${ }^{11}$ is best known from the KNMWT 16827 partial skeleton from Lomekwi, Kenya (Leakey 1982). Paracolobus mutiwa has been described as slightly more terrestrial in comparison to $P$. chemeroni, although not to the extreme observed in Theropithecus oswaldi (Harris et al. 1988; Ting 2001; Hlusko 2007). This derives from subtle postcranial differences between $P$. chemeroni and $P$. mutiwa, with $P$. mutiwa displaying slightly more cercopithecine-like features of the tarsus, pelvis, femur, glenoid fossa, and humerus (Harris et al. 1988; Ting 2001). Overall, the locomotor reconstruction for $P$. mutiwa is a mix of arboreal and terrestrial behaviors.

\footnotetext{
${ }^{11}$ Research in progress is examining the taxonomic status of Paracolobus mutiwa due to observed differences in craniodental morphologies with other Paracolobus fossils (Nina Jablonski, personal communication).
} 


\section{OBJECTIVES}

The primary objective of this chapter is to estimate range of hip abduction for fossil hominoids Rudapithecus hungaricus and Proconsul nyanzae. These fossil apes are compared to extant anthropoids in order to test their published locomotor reconstructions. As previously discussed, Rudapithecus hungaricus is hypothesized to have been highly suspensory and so it is expected that this taxon would have had a range of abduction similar to extant suspensory taxa. Proconsul nyanzae is hypothesized to have been an ambling above branch quadruped that did not use forelimb-dominant behaviors (Le Gros Clark and Leakey 1951; McHenry and Corruccini 1983; Walker and Pickford 1983; Beard et al. 1986; Ward 1993; Ward et al. 1993; Kelley 1997; Ward 2007), and would therefore be expected to have had a more limited range of abduction similar to extant non-suspensory taxa. It is expected that Rudapithecus hungaricus will have a range of abduction that is greater than that estimated for Proconsul nyanzae.

A secondary objective of this chapter is to estimate range of hip abduction for several large-bodied fossil cercopithecoids. Earlier chapters in this dissertation indicated that abduction ability in suspensory taxa is not a consequence of body size. The extant non-suspensory sample ${ }^{12}$ has a more limited range of body sizes than the suspensory $\operatorname{taxa}^{13}$, and so it is unknown whether hip abduction might actually vary in a wider range of body sizes in the non-suspensory group. Fossil hominoids Rudapithecus hungaricus and Proconsul nyanzae are compared to large-bodied fossil cercopithecoids (28-58 kg,

\footnotetext{
12 The mean body masses in non-suspensory species range from a small of $\sim 2 \mathrm{~kg}$ in female Cebus to a large of $21 \mathrm{~kg}$ in male Papio anubis (Smith and Jungers 1997). The size range of the non-suspensory species means is only $\sim 19 \mathrm{~kg}$.

${ }^{13}$ The mean body masses in suspensory species range from a small of $5 \mathrm{~kg}$ in female Hylobates lar to a large of $170 \mathrm{~kg}$ in male Gorilla gorilla (Smith and Jungers 1997). The size range of the suspensory species means is $\sim 165 \mathrm{~kg}$.
} 
see Table 1) as an additional test that abduction relates to locomotor behavior rather than body size in non-suspensory taxa. The fossil cercopithecoids examined are Theropithecus oswaldi, Paracolobus mutiwa, and Paracolobus chemeroni, which all have been reconstructed as non-suspensory taxa with varying amounts of terrestrial and arboreal behaviors. It is expected that large-bodied fossil cercopithecoids will have a low range of hip abduction due to their hypothesized non-suspensory behaviors, further demonstrating that body size is not a primary determinant of range of abduction (degrees) in non-suspensory taxa.

\section{SAMPLE}

The extant comparative sample is the laser scanned sample used for abduction simulations described in Chapter 4. Species, sexes, and sample sizes correspond to those in Table 4.1, following the locomotor classifications provided in Table 2.2.

A total of six fossil specimens are sampled (Table 5.1). The fossil sample includes Proconsul nyanzae (KNM-MW 13142), Rudapithecus hungaricus (unpublished pelvis and corresponding RUD 184 femur), Theropithecus oswaldi (Olduvai-MCKII and KNM-ER 866), Paracolobus chemeroni (KNM-BC 3), and Paracolobus mutiwa (KNM-WT 16827).

The Rudapithecus hungaricus pelvis and femur from Rudabánya, Hungary, are in the process of being formally described (Ward et al. 2008; Begun and Kordos 2011; Begun et al. 2012). The Rudapithecus hungaricus pelvis (undescribed, Figure 5.1) and femur (RUD 184, Figure 5.3) were found together during excavations in the summer of 2006 (Ward et al. 2008) and are associated with a female cranium (RUD200) (David Begun, personal communication). The original Rudabánya fossils are housed at the Geological Institute of Hungary (Budapest). Casts of the Rudapithecus hungaricus 
(unpublished) materials were graciously loaned by David Begun (Department of Anthropology, University of Toronto) and scanned by the author using a NextEngine scanner.

The Proconsul nyanzae (KNM-MW 13142) femur and pelvis belong to a partial skeleton from Mfangano Island, Kenya. The femur and pelvis have both been extensively described (Ward et al. 1993) and are figured here (Figure 5.1-5.2). The Proconsul nyanzae (KNM-MW 13142) original specimens were scanned by J. Michael Plavcan at the National Museums of Kenya (Nairobi) using a Konica-Minolta Vivid 9i.

The fossil cercopithecoid sample includes Theropithecus oswaldi (OlduvaiMCKII and KNM-ER 866), Paracolobus chemeroni (KNM-BC 3), and Paracolobus mutiwa (KNM-WT 16827). The T. oswaldi (KNM-ER 866) and P. chemeroni (KNM-BC 3) original specimens were scanned using a Konica-Minolta Vivid 9i by J. Michael Plavcan at the National Museums of Kenya. Theropithecus oswaldi (Olduvai-MCKII) was scanned by the author using a NextEngine scanner from high quality casts owned by Carol Ward. All fossil laser scan data were imported into PolyWorks IMEdit software for polygonal model creation as described for the extant sample in Chapter 4.

The abduction simulations described in Chapter 4 use digital best-fit techniques and relatively complete femora and pelves. The majority of fossil primate pelves and femora are incomplete and often cracked, crushed, abraded, weathered, or suffer from plastic deformation. Some of the fossils included in this study had pitting or abrasions that could be easily removed from the polygonal models. Because best-fit operations are known to be sensitive to surface irregularities (MacLatchy 1995; Hammond et al. 2013b), it was decided that small spot-cleaning of surface topography in the fossils would be more accurate that keeping the irregular surfaces. For instance, Proconsul 
nyanzae (KNM-MW 13142) has a pit on the articular surface of the femoral head probably resulting from carnivore toothmarks (Ward et al. 1993), but this small pit can be removed from the polygonal model and filled in using the automated hole-filling and repair features of the PolyWorks IMEdit software. All polygonal models from fossil scan data are figured (Figures 5.1-5.6).

\section{METHODS}

Maximum abduction was measured by (a) angular abduction of the femur relative to the midline plane and (b) by the physical distance between the knee and the midline plane. Angular abduction was measured in all six specimens. The abducted knee position could only be measured in Proconsul nyanzae (KNM-MW 13142). Although three specimens (KNM-MW 13142, Olduvai-MCKII, KNM-BC3) preserve the knee distally, only Proconsul nyanzae (KNM-MW 13142) could be measured relative to an original midline (discussed below).

Digital abduction methods differed from the procedures outlined in Chapter 4 in several ways to accommodate the use of fossils. First, repeated trials of measurements were undertaken on all fossils. This option was selected for the fossils because averaging values is known to reduce the influence of error that can result from a single measure (Lee 1990; Yezerinac et al. 1992).

Second, additional procedures for aligning incomplete fossil pelves (OlduvaiMCKII, KNM-ER 866, KNM-WT 16827, KNM-BC3, Rudapithecus unpublished) to the midsagittal plane were required because these fossils do not preserve the midline sympyseal and sacral morphology. These additional procedures are detailed below. 
Third, the Rudapithecus hungaricus acetabulum has some deformation cranially, with the lunate surface being broken or partially eroded in some places cranially and dorsally. Rather than using the entire lunate surface for sphere-fitting as described for the extant sample in Chapter 4, only the most caudal region of the lunate surface of the R. hungaricus hipbone was used for sphere-fitting (see Figure 5.8). The region used for sphere-fitting in R. hungaricus (Figure 5.8) corresponds to regions 6,7, and a portion of region 5 described in Hammond et al. (2013). Given that Hammond et al.'s study found mean percent prediction error of around 2\% (maximum 8\%) when using at least 2 regions of the lunate surface for sphere-fitting, it is expected that the $R$. hungaricus sphere-fitting technique has a similarly low potential for error in sphere fitting.

Lastly, Rudapithecus hungaricus, Proconsul nyanzae, and Paracolobus mutiwa preserve a pelvis but contralateral femur, so the femur of each was mirrored to match the same side as the pelvis. There is no known reason why substituting a right femur for a left femur would result in error, other than the potential for small differences in articular surface distribution within an individual, and thus the effects of substitution are expected to minimal.

\section{Fossils preserving midline morphology (Proconsul)}

Proconsul nyanzae (KNM-MW 13142) preserves enough sacral and pubic morphology for microscribe landmark data to be collected on the midline by articulating the sacrum with the hipbone. These landmark data were collected from cast and were provided by Carol Ward. The Proconsul nyanzae (KNM-MW 13142) pelvis was aligned to landmark data and the femora articulated following the methodology described in Chapter 4. The femur of Proconsul nyanzae (KNM-MW 13142) was re-articulated and measurements were collected 21 times. Averaging repeated trials of measurements 
should reduce the potential for error in a single measure (Lee 1990; Yezerinac et al. 1992), and 21 trials was selected to match the repeated sampling of the other fossil specimens (described below).

\section{Fossils without midline morphology (Rudapithecus, Theropithecus, Paracolobus)}

No sacra are preserved for Rudapithecus hungaricus (unpublished), Paracolobus mutiwa (KNM-WT 16827) and Theropithecus oswaldi (Olduvai-MCKII, KNM-ER 866), and therefore midline and lumbosacral microscribe landmark data cannot be acquired from these specimens directly. In addition, although a sacrum is preserved for Paracolobus chemeroni (KNM-BC 3), it is preserved in articulation with the right os coxa and shows evidence of deformation when the left os coxa is re-articulated. As such, midline and lumbosacral data for $P$. chemeroni (KNM-BC 3) were not acquired directly from the specimen. The scanned hipbones for $R$. hungaricus (unpublished), T. oswaldi (Olduvai-MCKII, KNM-ER 866), P. mutiwa (KNM-WT 16827) and P. chemeroni (KNMBC 3) were alternatively aligned relative to a midline plane using scaled coordinate data from multiple extant species to provide a range of likely estimates of pelvic orientations.

A range of pelvic orientations based on extant species was created for each incomplete fossil pelvis. This additional process was necessary to align the incomplete pelves to a midline plane. Five random females were selected from the extant sample of Pongo pygmaeus, Pan troglodytes, Symphalangus syndactylus, Hylobates lar, Papio cynocephalus, Nasalis larvatus, and Alouatta caraya (Table 5.4). These taxa were selected because they sample suspensory and non-suspensory taxa, which are thought to have different pelvic morphologies related to their different locomotor behaviors (see Ward 1991; 1993). Taxa were also selected specifically to sample different body sizes since it is currently unknown how certain portions of the pelvis change in response to 
size. The mean body weights from these species run from a low of $4.33 \mathrm{~kg}$ in female Alouatta caraya and a high of $45.8 \mathrm{~kg}$ in female Pan troglodytes (Smith and Jungers 1997). Only females were selected to eliminate error that might result from mixing male and female landmark data.

Species mean landmark configurations were generated for the sample of extant species in MorphoJ software (Klingenberg 2011). Configurations were generated using all 14 landmarks, and then again using the reduced sample of four landmarks (PIIS, dorsal acetabulum, center of acetabular fossa, and ischial spine). Two centroids were calculated from the mean configurations in order to calculate the scaling factor for the extant landmark data. The first centroid ("Centroid 1") consists of the landmarks that are present in the fossil pelves (PIIS, dorsal acetabulum, center of acetabular fossa, and ischial spine) whereas the second centroid ("Centroid 2") consists of all 14 landmarks collected from the extant species. The species, specimen numbers, and mean landmark centroid sizes are provided in Table 5.4.

The linear relationship between the mean size of all landmark data (14 landmarks) and the reduced number of landmarks preserved in fossils (4 of 14 landmarks) was then calculated. Centroid2 was plotted as a function of Centroid1 for extant species (Figure 5.7). Both a standard major axis (SMA) and least square regression (OLS) approach were used to estimate the linear relationship between centroids. The correlation coefficient is high $\left(r^{2}=0.939\right)$ and both models produce similar functions (SMA y=3.755x-9.315; OLS y=3.638x-3.079).

Centroids of the fossils were then calculated so that the extant mean landmark configurations could be scaled to the appropriate size for each fossil specimen. First, a centroid size was established for each fossil pelvis using three-dimensional landmark 
coordinates from the posterior inferior iliac spine (PIIS), dorsal acetabulum, center of acetabular fossa, and ischial spine. Microscribe landmark coordinates were collected by the author on Rudapithecus hungaricus (unpublished), Theropithecus oswaldi (OlduvaiMCKII, KNM-ER 866), Paracolobus mutiwa (KNM-WT 16827), and Paracolobus chemeroni (KNM-BC 3) from high quality casts housed in the Ward Lab (University of Missouri). Microscribe landmark coordinates were collected 5 times for each fossil. The anterior superior iliac spine was excluded from the landmark coordinates in the fossils as most fossils have a broken or damaged anterior ilium. These landmark coordinates were then imported into MorphoJ, where the mean landmark configuration was created using a procrustes fit algorithm (Table 5.2), and a centroid (Centroid1) was calculated for each specimen from mean from the 5 landmarking trials (Table 5.3).

Centroid2 for the fossils was estimated using both the SMA and OLS equations (Table 5.3), with predictions from both equations being virtually the same. The Centroid2 size predicted from SMA for each fossil was then used to numerically scale up the mean landmark configurations for each extant species. Mean landmarks for each fossil were multiplied by the predicted Centroid2 size estimated from SMA. In fossil cercopithecoids, pelvis landmark data for extant species was usually scaled up, whereas Rudapithecus hungaricus required scaling the landmarks down. Each scaled species mean landmark configuration was imported into IMInspect, where the midline and lumbosacral planes were created from the landmark data, as in Chapter 4.

Differences in the degree of femoral angular abduction could certainly result from different extant pelvic orientations (e.g., Pan vs. Papio vs. Ateles). However, different measures of angular abduction could also result from subtle differences in manual alignments of the femur within the acetabulum in a single species (e.g., trial 1 vs. trial 2 
vs. trial 3). In order to minimize this type of error, femora were abducted and bound to the pelvis before being aligned to different species mean landmark configurations. Specifically, each fossil femur was abducted within the acetabulum and then locked into a position of maximum abduction prior to any pelvic alignment procedures. This was repeated three times using duplicated femur models, providing three possible abducted femoral positions for each pelvis. By locking the abducted femora into place, this step ensured that femur position was held constant during all subsequent pelvic alignments so that measurements exclusively reflect the influence of pelvic alignment and not variability in femur positioning.

Fossil pelves were aligned to the midline planes and associated scaled extant landmarks, moving the three femora with them. The Rudapithecus, Paracolobus, and Theropithecus fossil pelves were then aligned to the PIIS, dorsal acetabulum, center of acetabular fossa, and ischial spine landmarks as described for the extant sample in Chapter 4. In total, Rudapithecus hungaricus (unpublished), Theropithecus oswaldi (Olduvai-MCKII, KNM-ER 866), Paracolobus mutiwa (KNM-WT 16827), and Paracolobus chemeroni (KNM-BC 3) fossils were each aligned to 7 species mean landmark configurations based on 3 trials of articulation. As a result, 21 configurations for femoral abduction were measured for each of these fossils. The mean value and range of the 21 configurations are reported for each fossil (Table 5.5). The fossil pelves aligned to scaled landmark data represent different orientations relative to the midline but not necessarily accurate distances from the midline, as pelvic breadth varies between taxa. As such, abducted knee position could not be measured in pelves aligned to scaled landmark data. 


\section{Quantitative Analyses}

Means, standard deviations, and ranges of angular abduction were calculated for the 21 repeated alignments for each fossil (Table 5.5). The abducted knee position could only be measured in Proconsul nyanzae (KNM-MW 13142). The means, standard deviations, and ranges of the Proconsul nyanzae (KNM-MW 13142) abducted knee position in the repeated trials were calculated. Fossil means were plotted alongside boxplots of extant data for visualization (Figures 5.3 and 5.4). Linear discriminant function analyses were used to predict locomotor classification for each fossil based on mean angular abduction value (from Table 5.6). Quantitative analyses were performed in $\mathrm{R}$ (version 3.0.1, R Core Team 2012), with DFA posterior probabilities calculated using the MASS package (Venables and Ripley 2002).

\section{RESULTS}

\section{$\underline{\text { Angular abduction (degrees) }}$}

Angular abduction was higher in Rudapithecus hungaricus (unpublished) than all other fossil catarrhines examined. The mean estimated maximum hip joint abduction for Rudapithecus hungaricus (unpublished) is $56^{\circ}$ (range $47-61^{\circ}$; Table 5.5 and Figure 5.9) and a discriminant function analysis predicts $R$. hungaricus is suspensory (78\% posterior probability, Table 5.6). This estimate of abduction for $R$. hungaricus is larger than all non-suspensory extant specimens except a single Macaca nemestrina male (MCZ 35670 , abduction $=57^{\circ}$ ). Rudapithecus hungaricus has lower abduction than observed in Pongo and probably Pan paniscus, and is most similar to that observed in Ateles and hylobatids. 
Proconsul nyanzae (KNM-MW 13142) would have been capable of less abduction than most suspensory taxa. The mean estimated maximum hip joint abduction for $P$. nyanzae (KNM-MW 13142) is $43^{\circ}$ (range $35-46^{\circ}$; Table 5.5 and Figure 5.9), a degree of abduction that overlaps with both locomotor groups. A discriminant function analysis finds a high likelihood that $P$. nyanzae (KNM-MW 13142) is nonsuspensory (73\% posterior probability, Table 5.6). The degree of angular abduction is consistent with above-branch quadrupedal locomotor behaviors in P. nyanzae (KNMMW 13142), although not necessarily reconstructions that include highly abducted hindlimb postures. The results for horizontal abduction in Proconsul nyanzae (KNM-MW 13142) are also consistent with previous work by MacLatchy and Bossert (1996), who estimated the range of abduction during extension in KNM-MW 13142 to be approximately $49^{\circ}$.

The mean estimated maximum hip joint abduction for Paracolobus chemeroni (KNM-BC 3 ) is $37^{\circ}$ (range $31-43^{\circ}$; Table 5.5). This value overlaps with suspensory Gorilla gorilla but is most consistent with non-suspensory cercopithecoids such as extant Theropithecus (i.e., geladas), Papio, and Cercopithecus (Figure 5.9). The linear discriminant function analysis finds a high likelihood that $P$. chemeroni (KNM-BC 3) is non-suspensory (88\% posterior probability, Table 5.6).

The mean estimated maximum hip joint abduction for Paracolobus mutiwa (KNMWT 16827 ) is $42^{\circ}$ (range $36-49^{\circ}$; Table 5.5). This value is similar to that observed in $P$. chemeroni (KNM-BC 3) and, accordingly, the interpretations are similar. Like $P$. chemeroni (KNM-BC 3), P. mutiwa (KNM-WT 16827) overlaps with suspensory Gorilla gorilla but is most consistent with non-suspensory cercopithecoids such as Theropithecus, Papio, and Cercopithecus (Figure 5.9). The linear discriminant function 
analysis finds a high likelihood that $P$. mutiwa (KNM-WT 16827) is non-suspensory (84\% posterior probability, Table 5.6).

The mean estimated maximum hip joint angular abduction for Theropithecus oswaldi (KNM-ER 866, Olduvai-MCKII) is $37^{\circ}$ (range $31-43^{\circ}$; Table 5.5). Abduction in specimen KNM-ER 866 (mean $28^{\circ}$ ) is low even for the non-suspensory taxa. Although both specimens technically overlap with Gorilla gorilla, they are most similar to nonsuspensory taxa (Figure 5.9). A linear discriminant function analysis predicts that $T$. oswaldi specimen KNM-ER 866 has a 97\% posterior probability of being nonsuspensory, with $T$. oswaldi specimen Olduvai-MCKII having a 76\% posterior probability of being non-suspensory.

The large-bodied fossil cercopithecoids have a low range of hip abduction, confirming that body size is not driving range of abduction (degrees) in extant nonsuspensory taxa. The fossil cercopithecoids display lower ranges of angular abduction compared to Rudapithecus hungaricus and, to a lesser extent, Proconsul nyanzae.

\section{Abducted knee position ( $\mathrm{mm}$ )}

As previously noted, the abducted knee position can only be measured in fossil taxa that preserve midline morphology because fossil pelves aligned to scaled landmark data do not necessarily accurate distances from the midline, as pelvic breadth varies between taxa. Accordingly, only values for Proconsul nyanzae (KNM-MW 13142) can be reported and compared to extant taxa. The mean abducted knee position for Proconsul nyanzae (KNM-MW 13142) is $260 \mathrm{~mm}$, which is intermediate between extant hylobatids and great apes for abducted knee position (Figure 5.10). In fact, a linear discriminant function analysis finds a $91 \%$ posterior probability that $P$. nyanzae (KNMMW 13142) is suspensory. These results, which align P. nyanzae (KNM-MW 13142) 
with large-bodied (i.e., suspensory) extant species, should be interpreted in consideration with both angular abduction and KNM-MW 13142 body size estimates (see discussion below).

\section{DISCUSSION}

\section{$\underline{\text { Hip abduction in Miocene hominoids }}$}

The results of this study suggest that Rudapithecus hungaricus was probably capable of hip postures with a high degree of abduction, and thus may have been adapted for suspensory forms of locomotion similar to the types of locomotion found in extant apes. The degree of abduction predicted for $R$. hungaricus (mean $56^{\circ}$ ) suggests that it would have achieved abducted hip positions most similar to Ateles and hylobatids, two smaller-bodied and highly suspensory extant taxa. Given that the in silico models of abduction conservatively estimate passive range of abduction in suspensory taxa, it is likely that Rudapithecus hungaricus was actually capable of a larger range of abduction when alive. This study corroborates previous interpretations primarily from phalangeal and carpal bones that R. hungaricus was a suspensory species (Begun 1992; 1993; Kivell and Begun 2009; Begun and Kordos 2011; Begun et al. 2012). Finding a range of abduction similar to extant suspensory taxa in Rudapithecus hungaricus demonstrates that inferences about suspensory locomotion can be made by modeling hindlimb joint function in extinct hominoids.

This study finds that hip joint abduction in Proconsul nyanzae (KNM-MW 13142) is not necessarily greater than non-suspensory cercopithecoids, which differs from most of the previous interpretations of the KNM-MW 13142 femoral morphology. Past work on $P$. nyanzae (KNM-MW 13142) has usually emphasized that the hip joint appears to have been more mobile than cercopithecoids based on an apparently high neck-shaft 
angle and spherical femoral head with even femoral head articular surface distribution (Ward 1991; 1993; Ward et al. 1993). If Proconsul nyanzae (KNM-MW 13142) did not have hip abduction like extant apes despite similarities in aspects of femoral morphology, this suggests that many of the features often used to infer higher hip mobility are not necessarily reflecting increased ranges of motion. Given the substantial variation in Miocene hominoid femora (see Chapter 2), identifying which morphologies are the best predictors of hip mobility is one of the most important next steps for interpreting functional abilities in isolated fossil hominoid femora. As proposed in Chapter 3, future work should directly quantify the relationship between hip joint mobility and hip morphologies, and explore what aspects of hip morphology are and are not related primarily to $\mathrm{ROM}$ or other functional factors.

Although apparently not capable of more acrobatic hip postures, Proconsul nyanzae (KNM-MW 13142) would have been capable of spanning gaps in the canopy by virtue of being large. At such a large body size ( $35 \mathrm{~kg}$ for KNM-MW 13142) (Ruff et al. 1989), it is likely that $P$. nyanzae was using slow, deliberate movements with powerful manual and pedal grips for stability during transfer between supports (Cartmill and Milton 1977; Begun et al. 1994; Kelley 1997; Rose 1997; Ward 2007), and was almost certainly not bounding and leaping the way smaller-bodied arboreal Old World monkeys do when crossing gaps. Based on abduction ability, this study is in agreement with the studies that propose that $P$. nyanzae was a cautious above-branch quadruped. However, Proconsul nyanzae is within the range of suspensory species for abducted knee position, a metric which is heavily influenced by body size (see Chapter 4). Although $P$. nyanzae would have been able to reach supports across canopy gaps because of its size, it did not have joint mobility indicative of an adaptation for moving acrobatically below-branch like extant apes. Accordingly, if smaller-bodied Proconsul species such as 
Proconsul heseloni had similar abduction ability as $P$. nyanzae, these would have been limited to less discontinuous canopy (i.e., smaller gaps) or would have been forced to cross gaps differently than $P$. nyanzae.

The intermediate abduction ability but large span at the knee in Proconsul nyanzae (KNM-MW 13142) underscores the discussion offered in Chapter 4, which emphasized the need to look at abduction (degrees) and abducted knee position relative to the midline $(\mathrm{mm})$ in combination with each other. It is likely that the female Rudapithecus hungaricus studied here, with a high range of angular abduction possible at the hip joint, would have had a larger span at the knee than non-suspensory monkeys of similar size. However, this female Rudapithecus hungaricus individual would have a smaller span at the knee compared to larger-bodied Proconsul nyanzae, extant great apes, and maybe even male Rudapithecus hungaricus given the high levels of postcranial sexual dimorphism in this species (e.g., Morbeck 1983; Bernor et al. 2004; Begun and Kordos 2011). Non-suspensory taxa have an isometric relationship between size and knee position and large non-suspensory taxa are predicted to have an abducted knee position equal to suspensory taxa (Chapter 4, and Figure 4.12), which have a negatively allometric relationship. It is therefore probable that the large-bodied fossil cercopithecoids would have had a span at the knee overlapping with suspensory taxa simply because the fossil cercopithecoids are so large, yet clearly some of these specimens would not have been capable of abducted hindlimb postures like those possible in suspensory species. Both abduction (degrees) and abducted knee position relative to the midline $(\mathrm{mm})$ should be used to inform interpretations regarding positional capabilities, and it should not be forgotten that having a large hindlimb spatial envelope by virtue of being large is quite different from having a large envelope due to high levels of hip joint mobility. 


\section{Hip abduction in Plio-Pleistocene fossil cercopithecoids}

The fossil cercopithecoids have a range of abduction most similar to nonsuspensory taxa but still overlap with both locomotor groups. Range of abduction in Theropithecus oswaldi specimen KNM-ER 866 approaches the lowest range observed in extant terrestrial monkeys. Theropithecus oswaldi specimen Olduvai-MCKII, Paracolobus chemeroni (KNM-BC 3), and Paracolobus mutiwa (KNM-WT 16827) overlap with arboreal monkeys and even Gorilla gorilla. The discriminant function results indicate that these species would be most likely classified as non-suspensory, with a very low probability that they would classify with suspensory primates (12\% probability in P. chemeroni, $16 \%$ in P. mutiwa, and $3-24 \%$ in T. oswaldi specimens).

There was a non-significant relationship between body size and hip abduction (degrees) reported in Chapter 4, but there was a small possibility that this result could have been affected by the size ranges available to be sampled within extant nonsuspensory taxa. The fossil cercopithecoids are as large as great apes and corroborate that body size does not determine hip abduction (degrees) in large cercopithecoids. Theropithecus oswaldi is similar in size to Pan or Pongo yet has a limited range of abduction in comparison. Paracolobus chemeroni (KNM-BC 3), and Paracolobus mutiwa (KNM-WT 16827) are smaller than T. oswaldi but have a similar range of abduction as T. oswaldi specimen Olduvai-MCKII.

The fossil cercopithecoids display abduction that falls into in a zone of overlap between locomotor extant groups. Technically, all of the fossil cercopithecoids fall into the interquartile ranges of Gorilla gorilla for degrees of abduction, and all but KNM-ER 866 have ranges that overlap with the range for Proconsul nyanzae (KNM-MW 13142) (see Table 5.5). This does not necessarily indicate that the fossil taxa were using the 
same locomotor behaviors as each other or even using the same behaviors as gorillas. One thing seems clear, however-- the range of hip abduction in the fossil cercopithecoids would not exclude suspensory behaviors but it would greatly reduce their arboreal versatility, particularly given their body size.

The estimates for hip abduction in these three large-bodied cercopithecoids seem in accord with other regions of the postcrania which suggest these species were adapted for terrestrial or semi-terrestrial behaviors. Rather than cross discontinuous gaps by suspension as in great apes, these large monkeys probably crossed gaps between tress by returning to the ground. This scenario is especially likely for KNM-ER 866, which displayed both limited abduction ability and large body size. Although Proconsul nyanzae (KNM-MW 13142) also was a large bodied species incapable of highly abducted hip postures, Proconsul nyanzae (KNM-MW 13142) had powerful manual and pedal grasping abilities which would have made above-branch transfer and bridging behaviors more stable at such a large body size.

Even though the abduction estimates for fossil cercopithecoids are low or only moderate at best, it is possible that the range of abduction estimated in the fossil cercopithecoids is actually too large. Abduction simulations in extant non-suspensory typically over-estimate the range of passive abduction measured in vivo (see Chapter 4), and so the fossil cercopithecoids simulations of abduction might be larger than they would have been in living animals. Additionally, Theropithecus oswaldi has a "reverse carrying angle of the femur" (Krentz 1993) which probably increased the angle formed between the femoral neck and the shaft in this species. Whether the abduction estimated for the fossil cercopithecoids is too large is unknown but it suggests that, if anything, the range of abduction in these species would have been even more restricted. 


\section{Evolutionary scenarios}

Fossil hominoids seem to become specialized for either more arboreal or more terrestrial behaviors during their evolutionary history. A model of hominoid locomotor evolution proposed by Nakatsukasa and Kunimatsu (2009) proposed that the earliest apes relied on slow pronograde quadrupedalism and began incorporating vertical climbing and other antipronograde behaviors (e.g., orthograde clamber, transfer, and bridging), until eventually there was a significant reliance on suspension in late Miocene hominoids (Figure 5.11). The Nakatsukasa and Kunimatsu (2009) model is placed in the context of a global cooling event which begins in the middle Miocene, where multileveled canopy forests were replaced by more fragmented and single-canopied forests. In this scenario, arboreal hominoids would have either opted to become more terrestrial to cross gaps in the canopy (e.g., Sivapithecus and Dryopithecus) or they would have become specialized for suspensory behaviors to cross gaps in the canopy (e.g., Rudapithecus). Features of the forelimb and hindlimb that facilitate gap crossing and suspensory behaviors (e.g., antipronograde climbing, clambering, transfer, bridging, brachiation, etc.) would thus be selected for in the more arboreal middle and late Miocene apes. Moreover, it is proposed here that suspensory species routinely using the flexible, small-diameter supports found in the terminal branch environment would be particularly likely to show high joint mobility as compared to suspensory species using the center of the crown where supports are larger and more rigid.

Elongated limbs are hypothesized to facilitate climbing on large-diameter supports (Cartmill 1974; Fleagle et al. 1981b; Cartmill 1985; Isler and Thorpe 2003) and so the range of knee positions within suspensory anthropoids might very well reflect different substrate usage. It remains to be seen how the spatial envelope relates to use 
of the environment, specifically how it might reflect preference for different forest canopy levels, types of arboreal supports, tree density, tree crown diameter, and/or continuity of the canopy (Figure 5.12). A horizontally continuous canopy is unlikely to necessitate antipronograde behaviors, where gaps can be crossed by simple reaching or short leaps, and high abduction ability and large spatial envelope could signify a shift towards more discontinuous canopy structure experienced by some Miocene hominoids. Although locomotor behaviors and canopy usage are often discussed in extant species (e.g., Cannon and Leighton 1994), the environmental context of fossil taxa is usually poorly integrated into discussions of locomotor behavior. Future work should investigate the relationship between spatial envelope in extant species and the environmental composition (Figure 5.12), as it may give insight into the relationship between the changing climate in the middle and late Miocene and how that relates to the highly derived locomotor morphology in the surviving relict populations.

The findings regarding both the fossil cercopithecoids are important for scenarios regarding catarrhine evolution more broadly. Much work has favored a scenario where arboreal behaviors in cercopithecoids evolved in the Plio-Pleistocene because many fossil colobines and cercopithecines appear semi-terrestrial or even fully-terrestrial (Andrews 1982; Harrison 1989; Ciochon 1993; Benefit 1999; Leakey et al. 2003). However, it has been proposed that most fossil colobines were arboreal by the Pliocene (Hlusko 2006), with the more terrestrially-adapted fossil colobines potentially being secondarily derived for terrestrial behaviors. More recently, arboreal adaptations have been found in Miocene fossil colobines at Lemudong'o (6 Ma, Hlusko 2007) and Nakali (9.8 Ma, Nakatsukasa et al. 2010) in Kenya. It is hypothesized that monkeys and apes competed for resources during the course of their evolution, in part because this earlier timing for colobine diversification in the middle to late Miocene coincides with the decline 
in Miocene hominoids in Africa (Nakatsukasa et al. 2010). Further research on both locomotor and dietary adaptations of both fossil hominoids and cercopithecoids should help identify how both groups might have directly competed during their evolutionary histories.

One of the biggest obstacles to developing and testing evolutionary scenarios about locomotor evolution is that we have yet to determine how different fossil taxa are related. Past workers attempting to resolve the Miocene phylogeny have found equally parsimonious tree topologies within a single analysis (Begun et al. 1997a; Rae 1999; Young and MacLatchy 2004). In addition, the most informative taxa for the evolution of suspensory behaviors have undergone substantial generic revisions in the last decade (see discussion of Hispanopithecus and Rudapithecus in Chapter 2), and most of these taxa are not included or accurately represented in these past phylogenies. Although taxa like Hispanopithecus and Rudapithecus are almost certainly within crown hominoids, if not potentially pongines or hominines (see Chapter 2), how these taxa are related to each other and other Miocene hominoids is unclear. Several small-bodied catarrhines potentially have suspensory adaptations as well, and interpretations vary as to whether species such as Dendropithecus and Limnopithecus are hominoids (Andrews and Simons 1977; Harrison 2010). It could even be argued that we do not know which species are within the crown group because of uncertainties regarding the timing of hylobatid evolution (Raaum et al. 2005; Matsudaira and Ishida 2010; Chan et al. 2012). If hylobatids diverged greater than $18 \mathrm{Ma}$ ago, it is possible that Proconsul could actually be an early hominid (i.e., stem great ape), which would require substantial reinterpretations of locomotor evolution in hominoids. Renewed investigations into the phylogenetic relationships between fossil catarrhines and extant hominoid taxa are needed. 
Gaps in the hominoid fossil record also limit efforts to reconstruct how and when suspensory behaviors appear. The first suspensory behaviors in Miocene apes appear in the late Miocene in Europe but we still do not have conclusive evidence for how and when apes entered Europe, as well as which locomotor adaptations characterized the earliest apes out of Africa. The earliest hominoid taxon currently known in Europe, Griphopithecus (16.5 Ma per Heizmann and Begun 2001; Begun et al. 2003a; Begun et al. 2003b), has limited postcranial materials suggesting generalized quadrupedalism consistent with mixed semi-terrestrial and semi-arboreal behaviors (Ehrensberg 1938; Begun 2002; Ersoy et al. 2008; Alba et al. 2010b). This locomotor reconstruction for early European apes is not very different from the clambering quadrupedalism of early Miocene hominoids like Proconsul. Later Pierolapithecus catalaunicus (11.9 Ma) has morphology more advanced than Proconsul, having modifications to the lumbar vertebrae, ribs, and pelvis that suggest more anti-pronograde behaviors (Moyà-Solà et al. 2004; Begun and Ward 2005; Alba et al. 2010a; Susanna et al. 2010; Hammond et al. 2013a) but has phalangeal and carpal morphology that is inconsistent with suspension (Moyà-Solà et al. 2004; Almécija et al. 2007; Almécija et al. 2009; Alba et al. 2010a). The other middle Miocene fossils apes (e.g., Equatorius, Sivapithecus and Dryopithecus) appear terrestrial and were probably not suspensory. Identifying how and when great apes evolved suspensory locomotor behaviors will require the recovery of additional middle and late Miocene fossil hominoids. Filling in the gap in the European hominoid fossil record between more primitive hominoids (i.e., Griphopithecus) and highly specialized late Miocene hominoids (i.e., Rudapithecus and Hispanopithecus) is another critical step needed to testing evolutionary scenarios of locomotor evolution in hominoids. 


\section{CONCLUSIONS}

The primary goal of this thesis is to determine whether adaptations for suspensory behaviors can be inferred in fossil hominoids based on hip joint abduction (see Chapter 1), and these results provide the first evidence of suspensory locomotor behaviors in a fossil ape based on hip joint mobility. Rudapithecus hungaricus, hypothesized to be highly suspensory based on forelimb elements, demonstrates that adaptations for suspensory behaviors can be inferred in fossil nonhuman hominoids from the hip joint based on range of abduction. The mean for angular abduction in Rudapithecus hungaricus was exclusively in the range of extant suspensory anthropoids and was found to have a high posterior probability of being suspensory in a discriminant function test. The abduction ability of Rudapithecus hungaricus was not within the range of highly acrobatic and quadrumanous Pongo, and instead fell closer to Ateles and hylobatids.

Proconsul nyanzae and the large fossil cercopithecoidsTheropithecus oswaldi, Paracolobus mutiwa, and Paracolobus chemeroni have substantially lower hip abduction than Rudapithecus hungaricus. Proconsul nyanzae was most similar to non-suspensory taxa for angular abduction and was only within the range of a single suspensory species (Gorilla gorilla). Proconsul nyanzae had a high posterior probability of being nonsuspensory. Despite a somewhat limited range of abduction, Proconsul nyanzae was able to position its knee relatively far from the midline by virtue of being large. Proconsul nyanzae would have reached across gaps in the canopy by virtue of being large but not necessarily through acrobatic hindlimb postures.

Rudapithecus hungaricus has an increased range of hip abduction relative to the other fossil species examined, potentially the result of directional selection for enhanced 
hindlimb mobility associated with more antipronograde arboreal behaviors than in early hominoids like Proconsul. Additional work disentangling evolutionary relationships between taxa, as well as the recovery of additional middle and late Miocene hominoid postcranial materials, would help clarify the timing and trajectory of suspensory behaviors. 


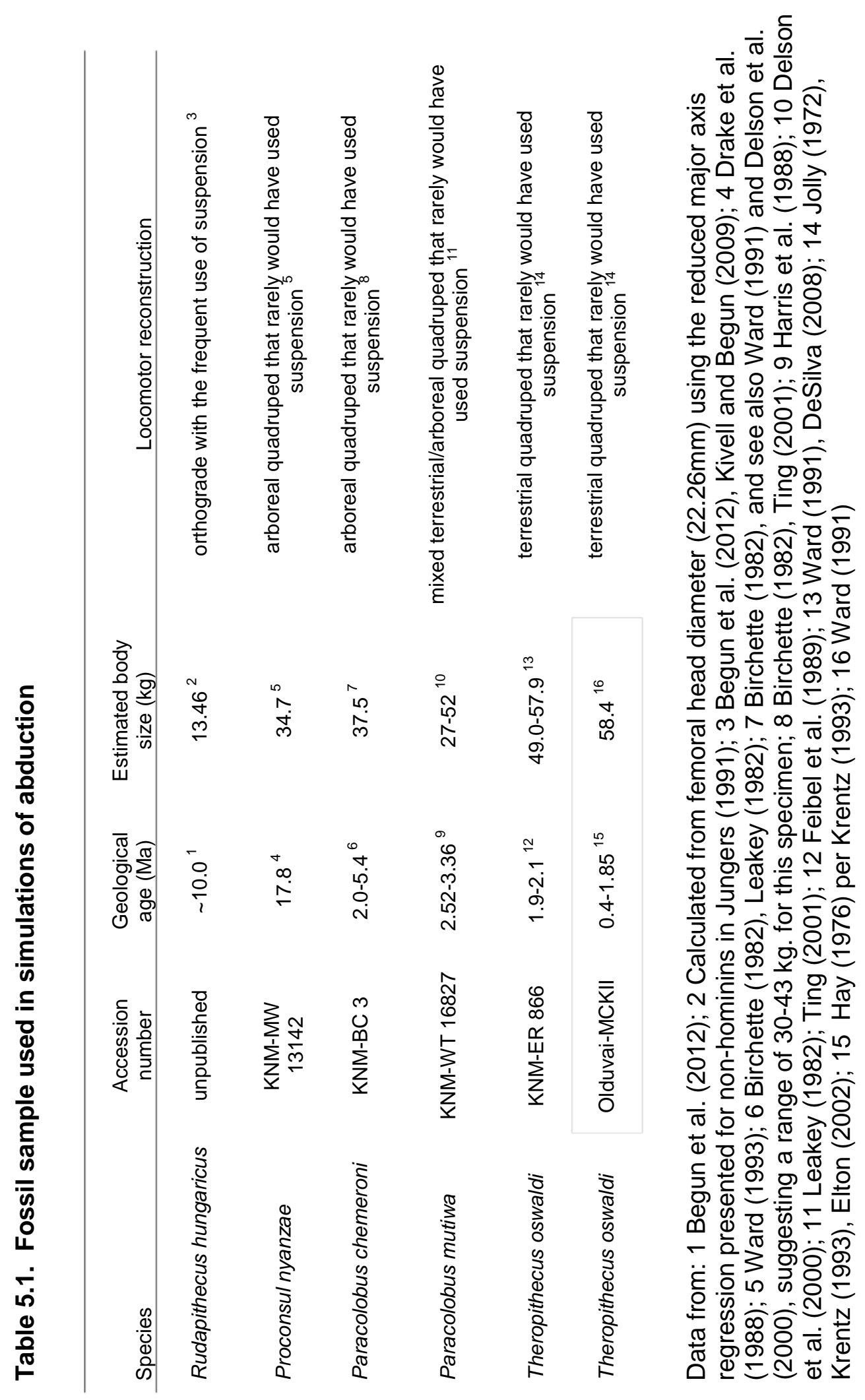




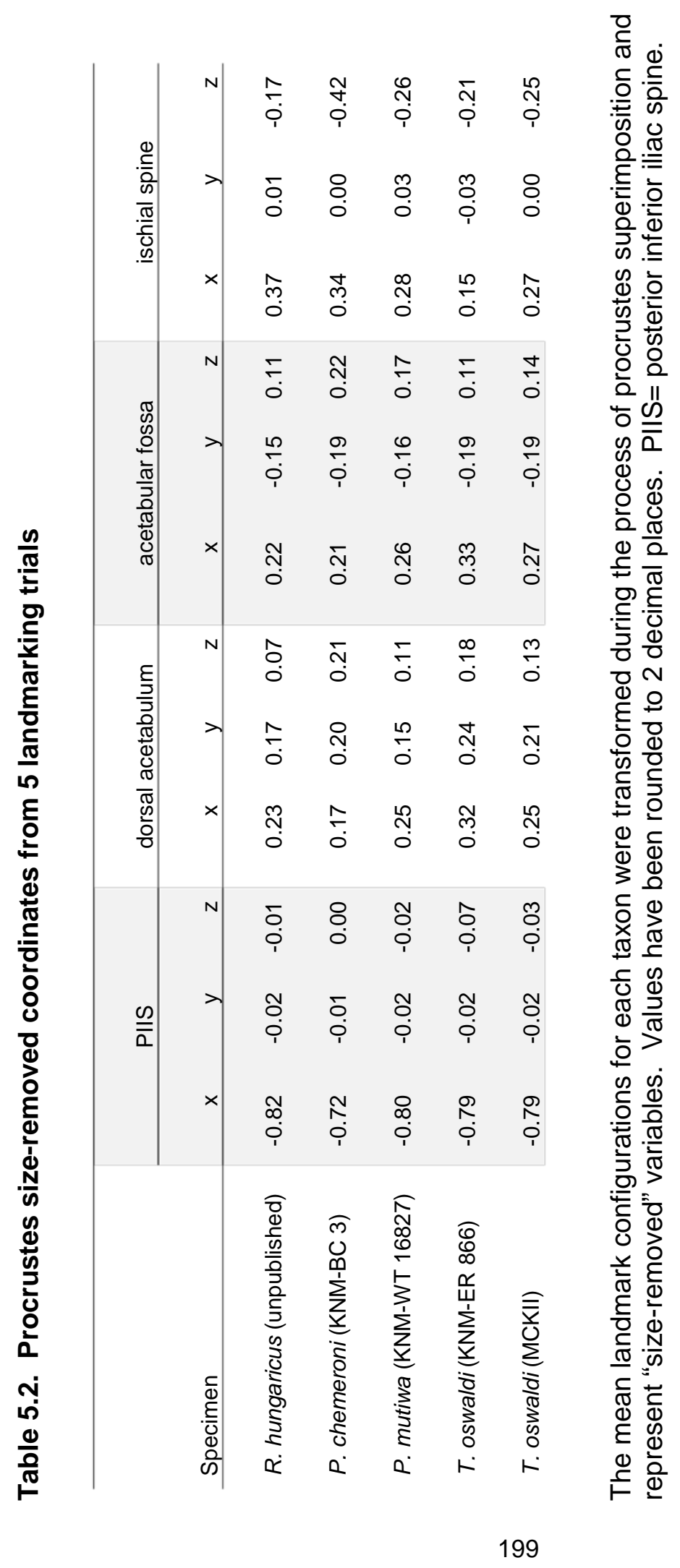


Table 5.3. Fossil Centroid1 and Centroid2 values from 5 landmarking trials

\begin{tabular}{lccc}
\hline Specimen & $\begin{array}{c}\text { Centroid1 } \\
\text { size }\end{array}$ & $\begin{array}{c}\text { Predicted } \\
\text { Centroid2 size } \\
\text { (SMA) }\end{array}$ & $\begin{array}{c}\text { Predicted } \\
\text { Centroid2 size } \\
\text { (OLS) }\end{array}$ \\
\hline R. hungaricus (unpublished) & 56.56 & 203.07 & 202.69 \\
P. chemeroni (KNM-BC 3) & 56.90 & 204.35 & 203.92 \\
P. mutiwa (KNM-WT 16827) & 70.99 & 257.26 & 255.19 \\
T. oswaldi (KNM-ER 866) & 65.63 & 237.14 & 235.70 \\
T. oswaldi (MCKII) & 70.14 & 254.04 & 252.07
\end{tabular}

Centroid1 was calculated from 5 landmarking trials. Centroid2 was estimated by inputting Centroid1 into the standard major axis regression (SMA) equation for the extant species $(y=3.755 x-9.315)$. Centroid values have been rounded to 2 decimal places. 
Table 5.4. Extant centroid sizes and specimens used to calculate centroids

\begin{tabular}{|c|c|c|c|}
\hline Species & $\begin{array}{l}\text { Centroid1 } \\
\text { size }\end{array}$ & $\begin{array}{l}\text { Centroid2 } \\
\text { size }\end{array}$ & Specimens Used* \\
\hline Pan troglodytes & 79.88 & 284.11 & $\begin{array}{l}\text { CMNH 1748, 1766, 1843, 3539, } \\
3551\end{array}$ \\
\hline Pongo pygmaeus & 70.48 & 272.69 & $\begin{array}{l}\text { USNM 145308, 145309; CMNH } \\
1443,1055,1167\end{array}$ \\
\hline $\begin{array}{l}\text { Symphalangus } \\
\text { syndactylus }\end{array}$ & 51.23 & 175.16 & $\begin{array}{l}\text { USNM 141161, 141162, } 271048 \\
\text { AMNH 106584; ZSM 1904.21 }\end{array}$ \\
\hline Hylobates lar & 46.81 & 148.18 & $\begin{array}{l}\text { MCZ 41412, 41424, 41442, } \\
41440,41494\end{array}$ \\
\hline Nasalis larvatus & 40.58 & 152.61 & $\begin{array}{l}\text { MCZ 37328, } 37339,37343, \\
41554,41559\end{array}$ \\
\hline Papio cynocephalus & 50.54 & 166.99 & $\begin{array}{l}\text { KNM 7264, 7262, 7254, 7276, } \\
7277\end{array}$ \\
\hline Alouatta caraya & 34.49 & 139.31 & $\begin{array}{l}\text { AMNH 211495, 211501, 211506, } \\
211512,215060\end{array}$ \\
\hline
\end{tabular}

*All specimens are female. Values in table have been rounded to 2 decimal places. 


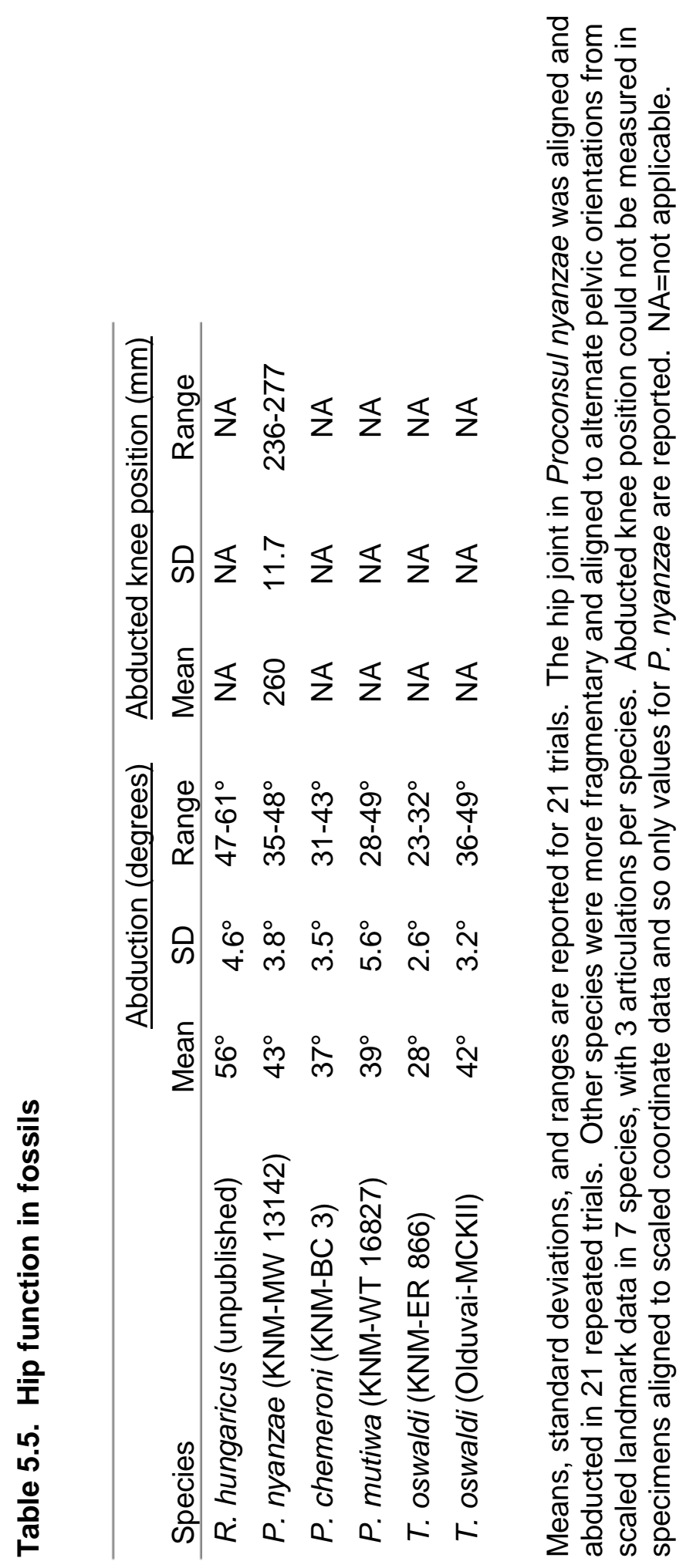


Table 5.6. Discriminant function percent classification of fossil means in extant suspensory and non-suspensory locomotor groups

\begin{tabular}{crr}
\hline & $\begin{array}{l}\text { Suspensory } \\
\text { taxa }\end{array}$ & $\begin{array}{l}\text { Non-suspensory } \\
\text { taxa }\end{array}$ \\
\hline Abduction (degrees) & & \\
\hline R. hungaricus (unpublished) & $78 \%$ & $22 \%$ \\
P. nyanzae(KNM-MW 13142) & $27 \%$ & $73 \%$ \\
P. chemeroni (KNM-BC 3) & $12 \%$ & $88 \%$ \\
P. mutiwa (KNM-WT 16827) & $16 \%$ & $84 \%$ \\
T. oswaldi (KNM-ER 866) & $3 \%$ & $97 \%$ \\
T. oswaldi (Olduvai-MCKII) & $24 \%$ & $76 \%$ \\
Abducted knee position (mm) & & $9 \%$
\end{tabular}




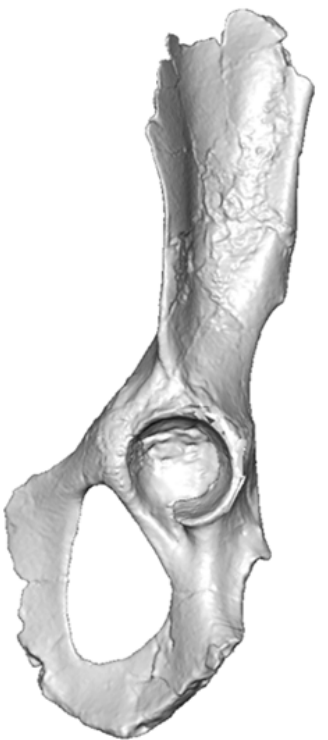

P. nyanzae

(KNM-MW 13142)

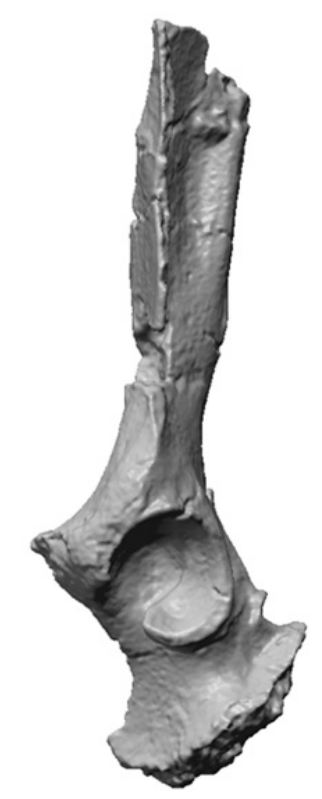

P. mutiwa

(KNM-WT 16827)

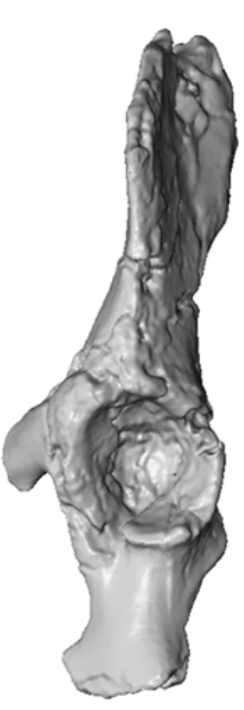

R. hungaricus (undescribed)

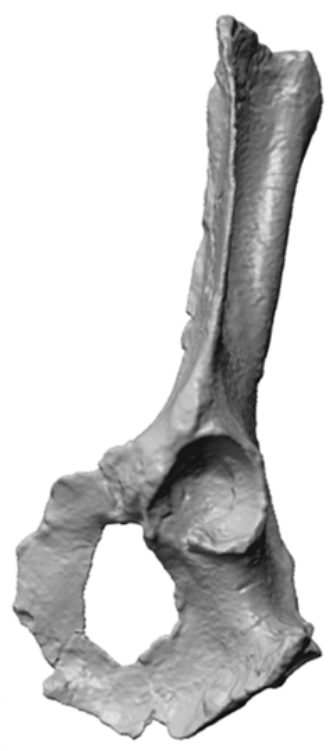

P. chemeroni (KNM-BC 3)

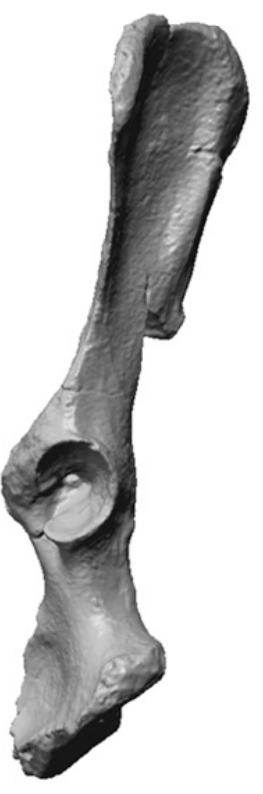

T. oswaldi (KNM-ER 866, mirrored)

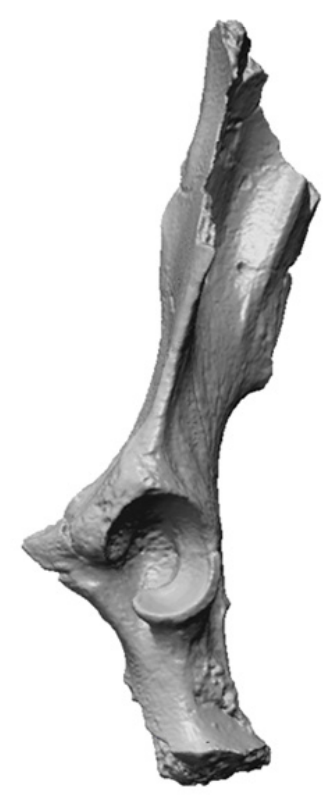

T. oswaldi (Olduvai MCK-II)

Figure 5.1. Polygonal models of fossil hominoid and cercopithecoid pelves used in simulations. 

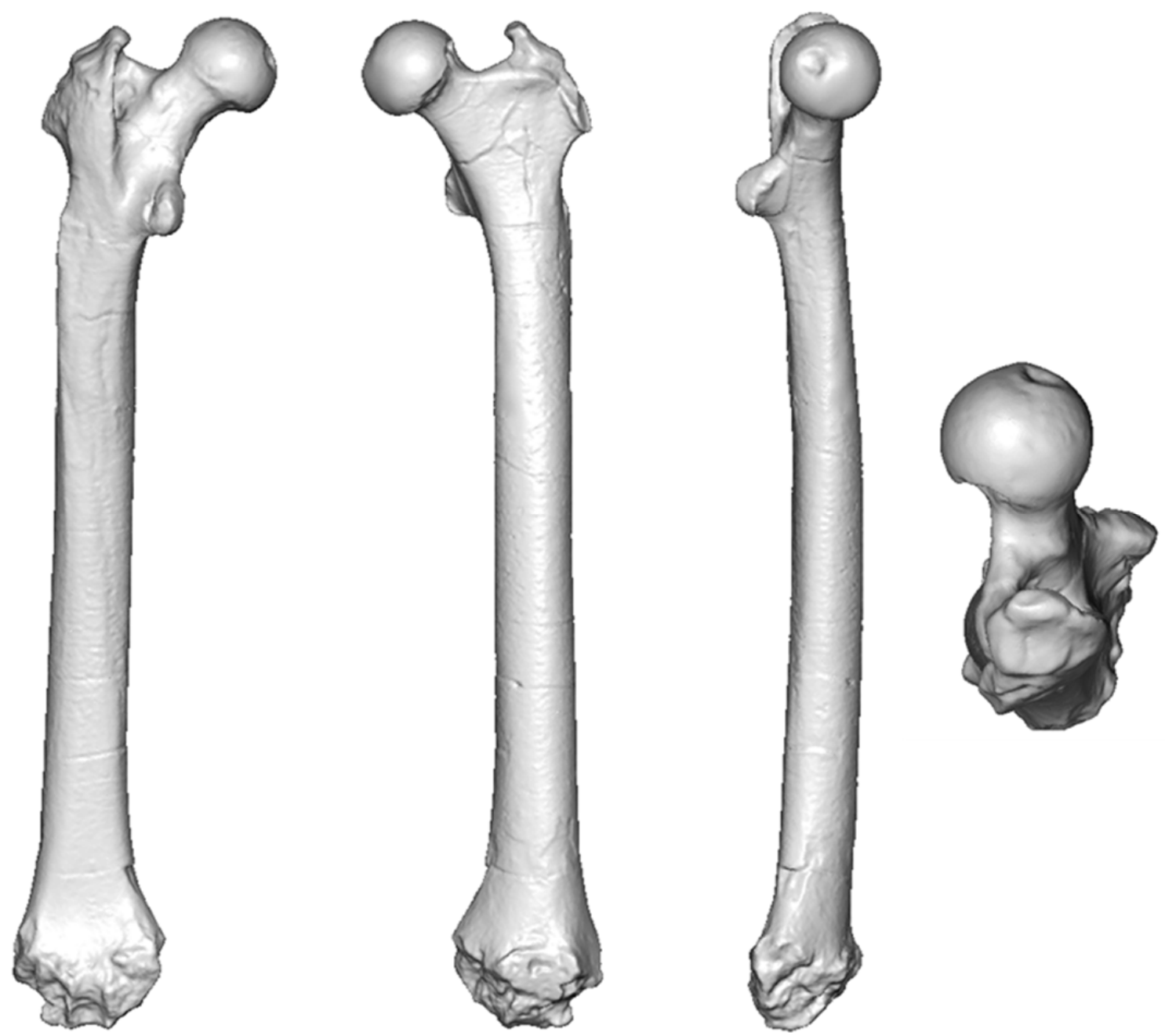

Figure 5.2. Polygonal model of the femur of Proconsul nyanzae (KNM-MW 12142) From left to right, posterior, anterior, medial, and superior views are shown. All views are not to the same scale. 

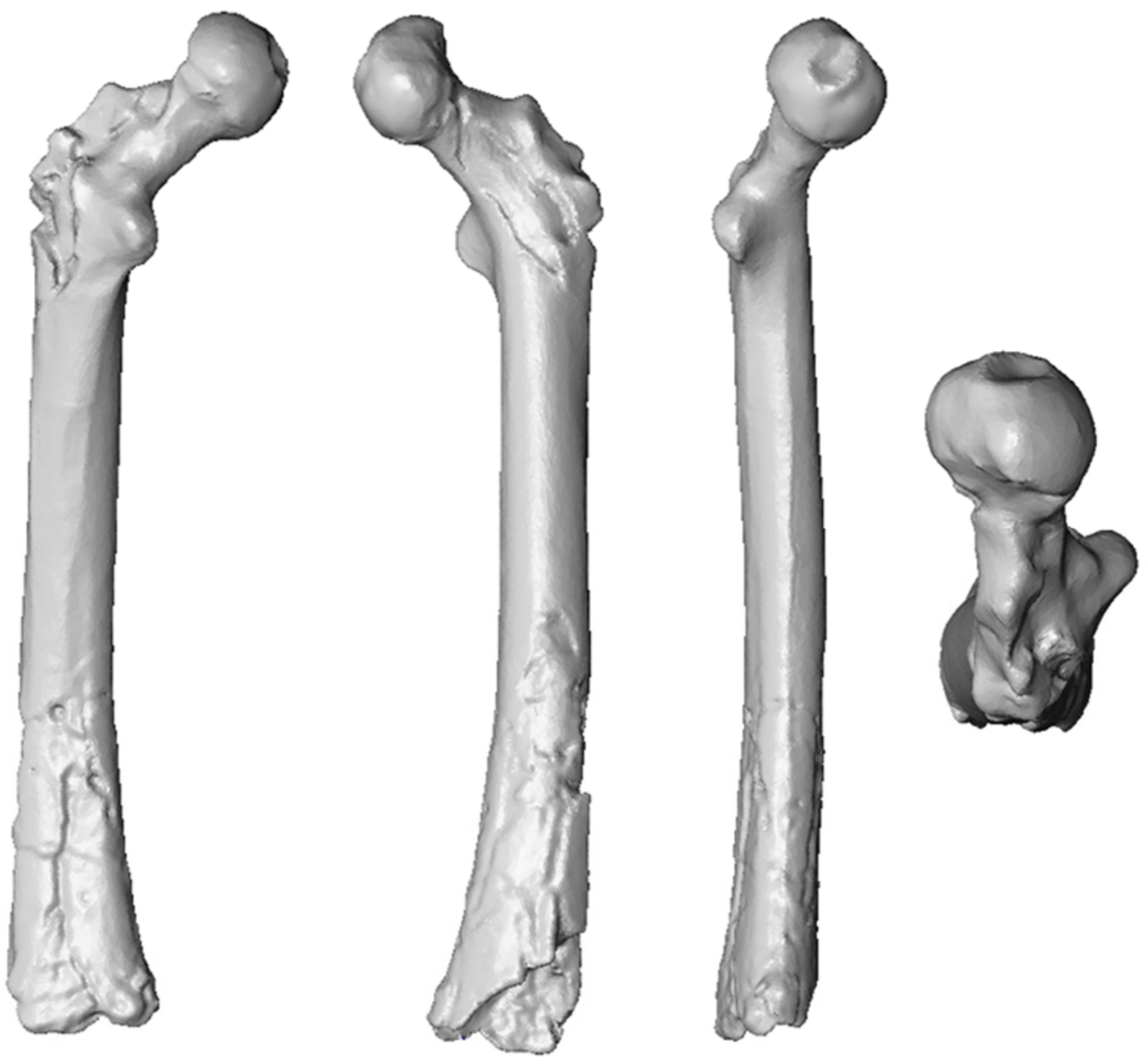

Figure 5.3. Polygonal model of the femur of Rudapithecus hungaricus (RUD 184) From left to right, posterior, anterior, medial, and superior views are shown. The RUD 184 femur is a right that has been digitally mirrored. All views are not to the same scale. 
(a) KNM-ER 866
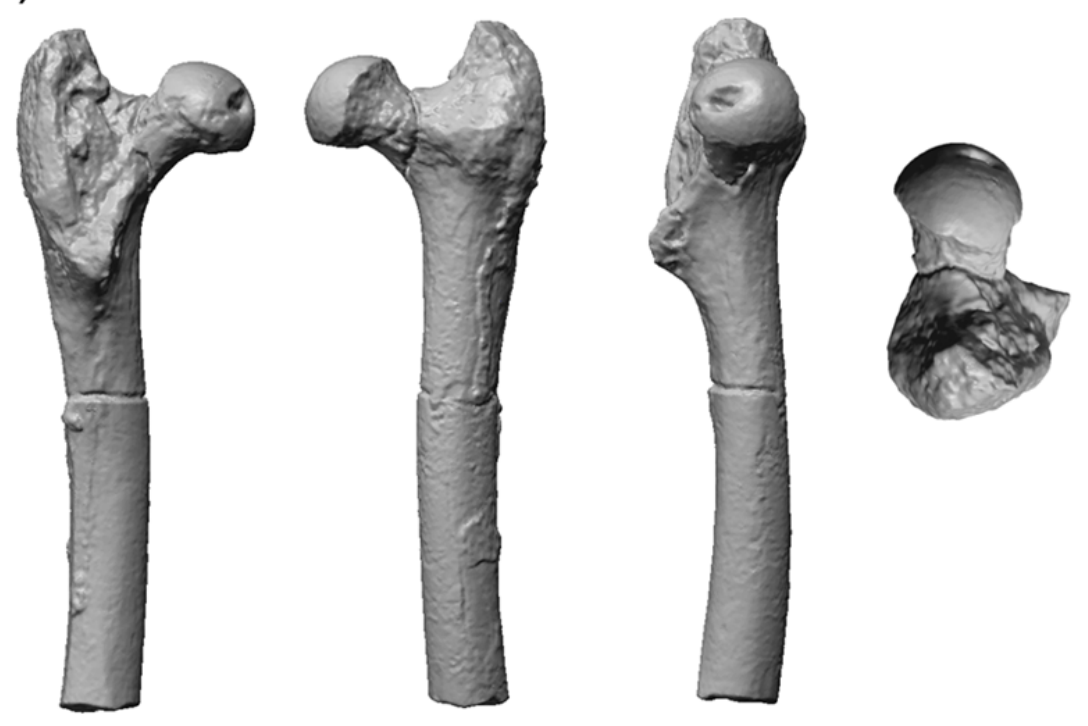

(b) Olduvai MCK-II
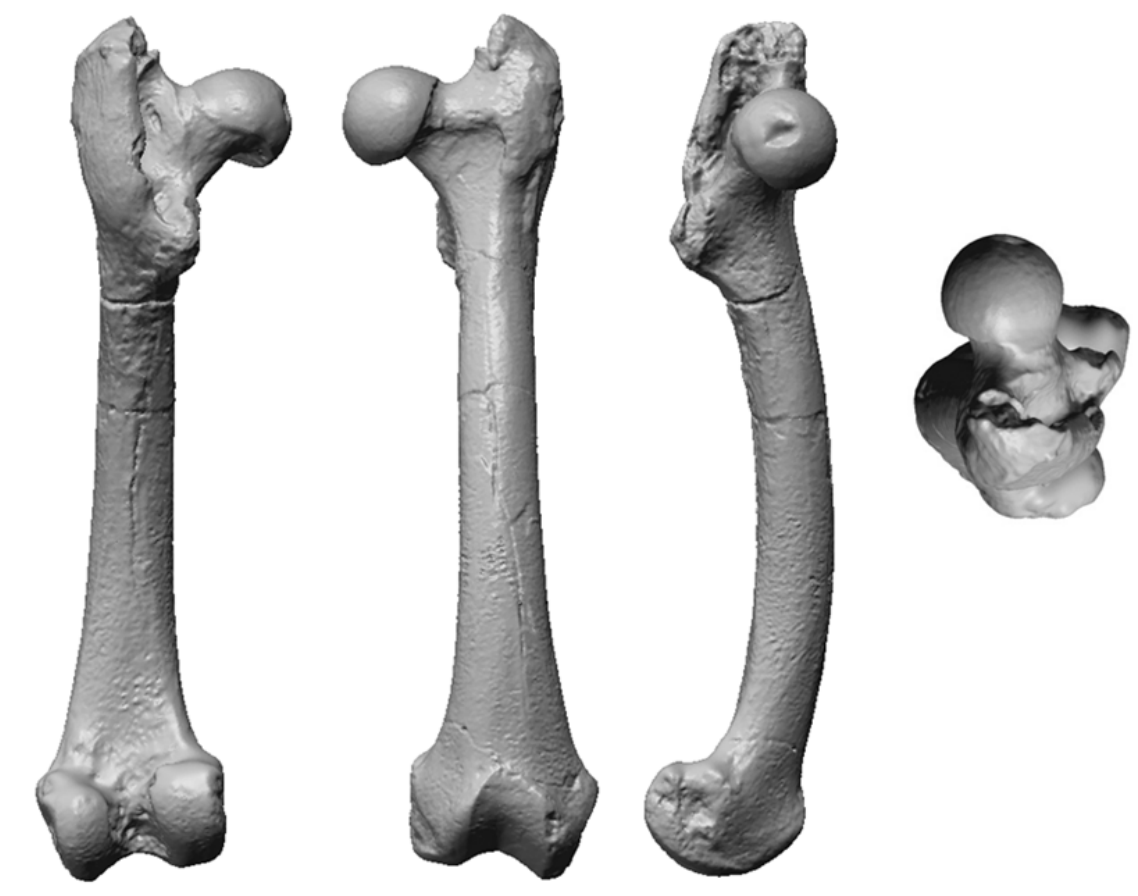

Figure 5.4. Polygonal models of the femur of Theropithecus oswaldi (KNM-ER 866, Olduvai MCK-II)

From left to right, posterior, anterior, medial, and superior views are shown. The KNMER femur is a right that has been digitally mirrored to maintain consistency in measurement technique. All views are not to the same scale. 

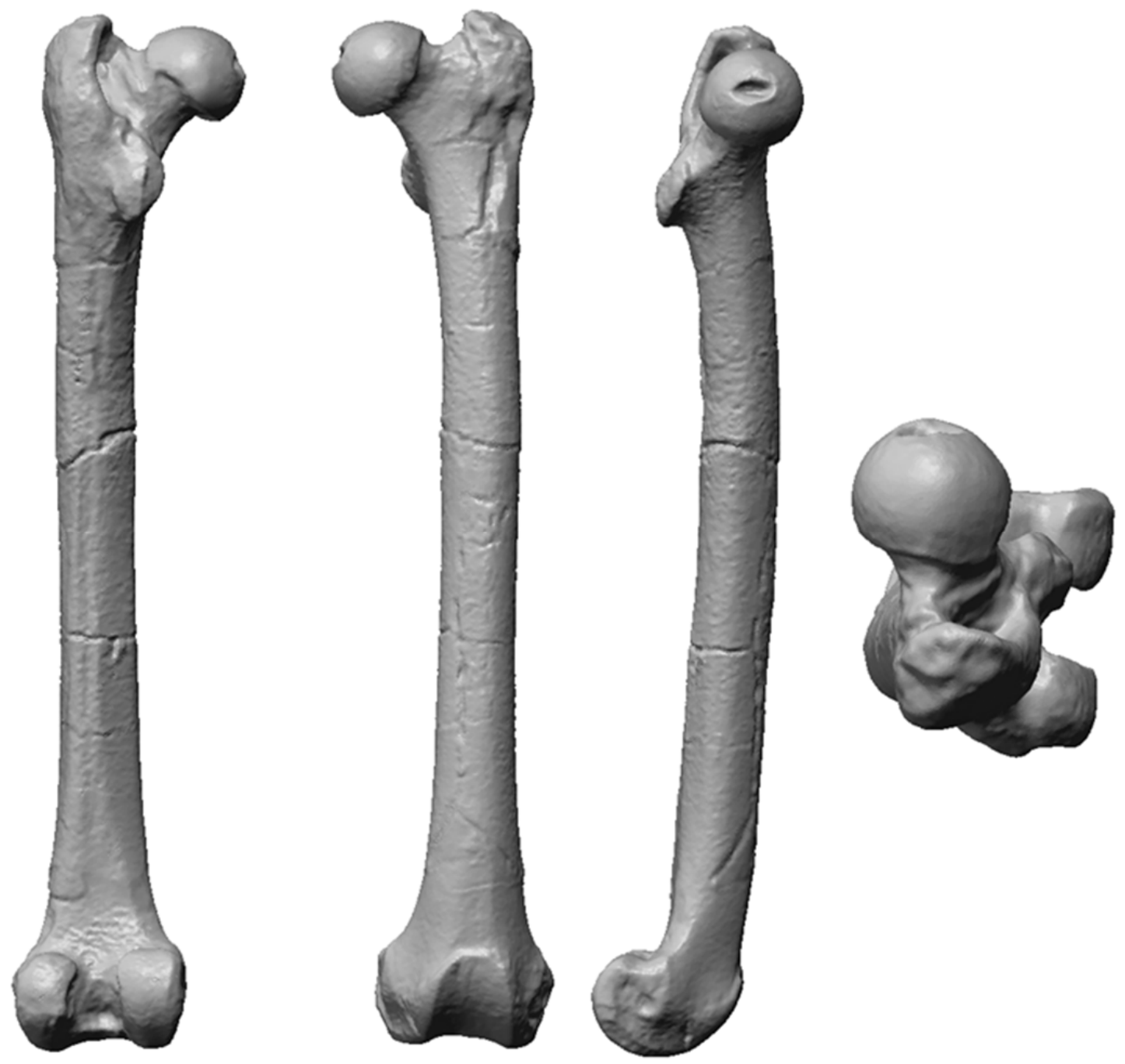

Figure 5.5. Polygonal models of the femur of Paracolobus chemeroni (KNM-BC 3) From left to right, posterior, anterior, medial, and superior views are shown. All views are not to the same scale. 

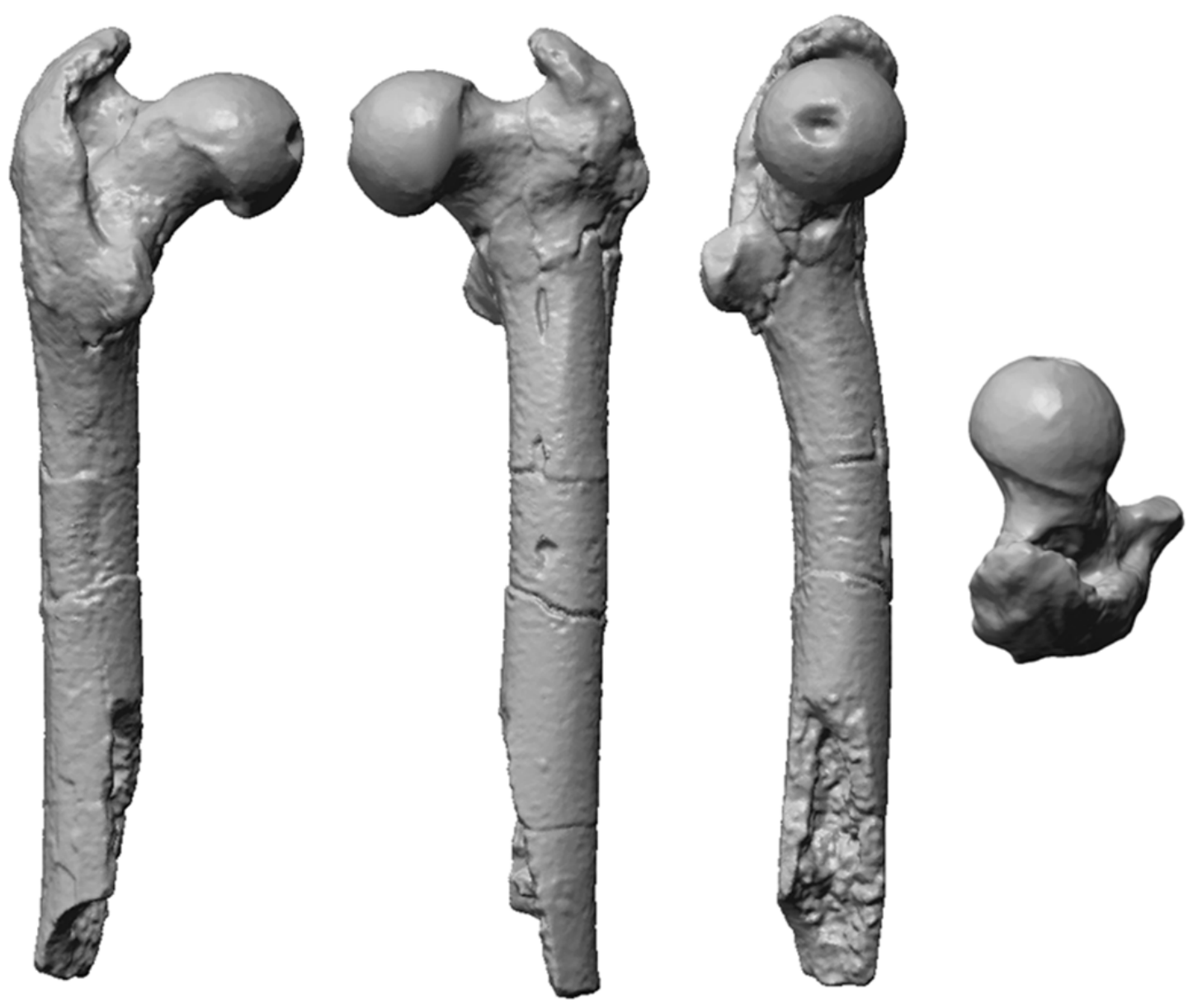

Figure 5.6. Polygonal models of the femur of Paracolobus mutiwa (KNM-WT 16827)

From left to right, posterior, anterior, medial, and superior views are shown. All views are not to the same scale. 


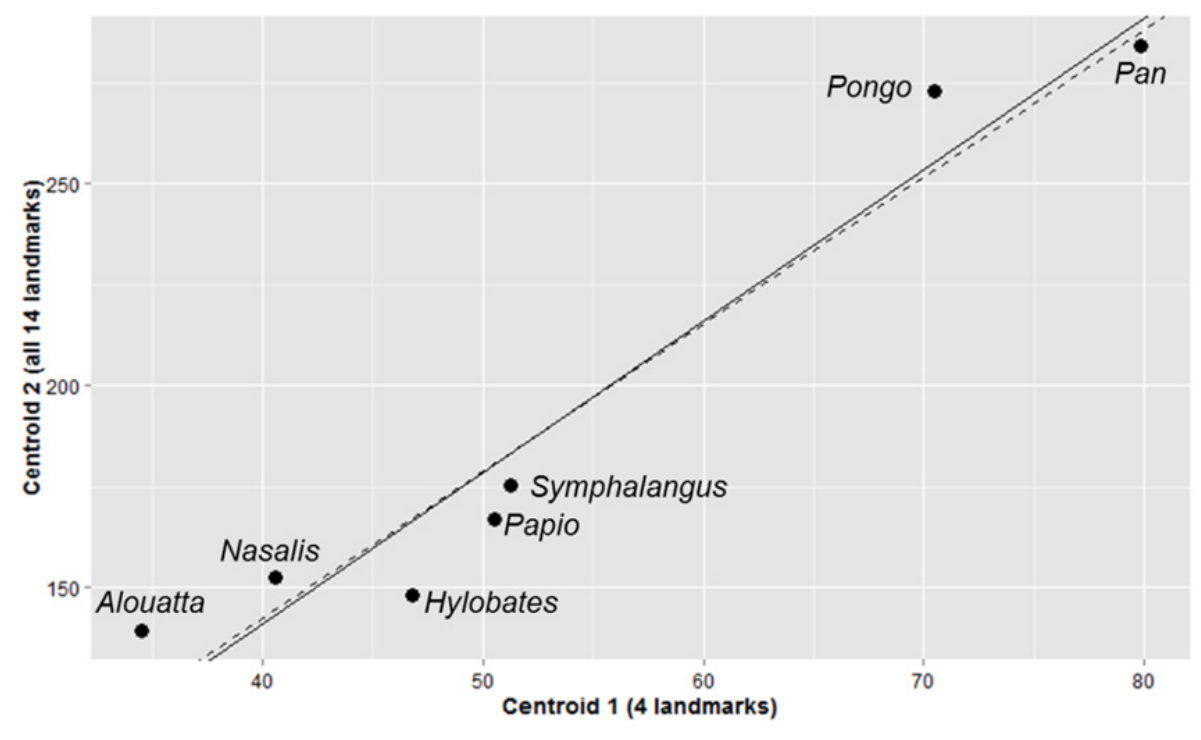

Figure 5.7. Centroid 2 (using 14 landmarks) as a linear function of Centroid 1 (using 4 landmarks)

Reduced major axis (solid line, $y=3.755 x-9.315)$ and least squares regressions (dotted line, $y=3.638 x-3.079$ ) are shown. Regression correlation coefficients are $r^{2}=0.939$. 

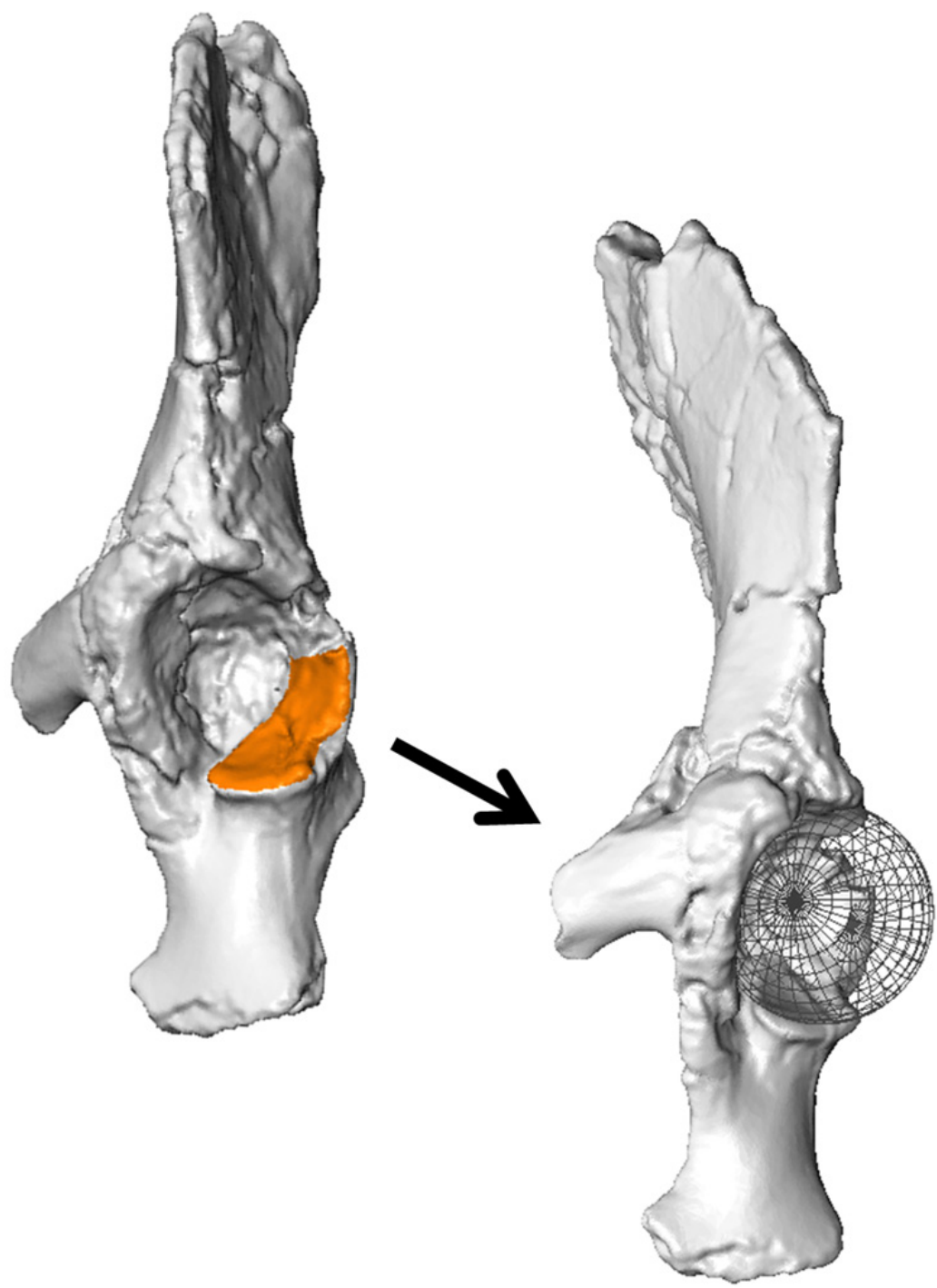

Figure 5.8. The region of the Rudapithecus pelvis used for acetabular spherefitting

The region used for sphere-fitting in $R$. hungaricus, indicated in orange, corresponds to regions 6,7, and a portion of region 5 described in Hammond et al. (2013). 


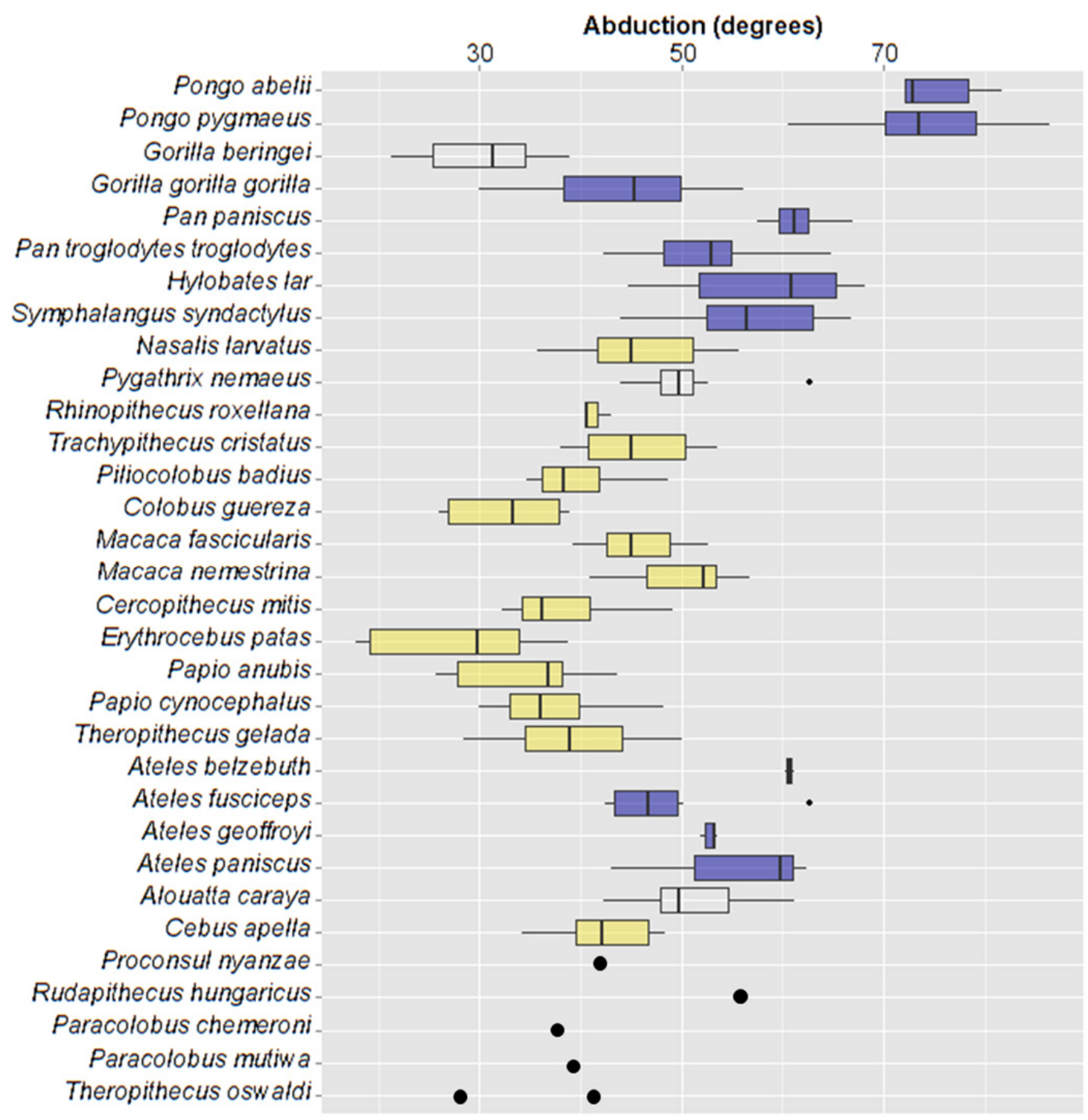

Figure 5.9. Mean abduction in fossil specimens plotted alongside boxplots of extant taxa

Suspensory taxa are indicated by the darker blue shaded boxplots and non-suspensory taxa are indicated by yellow boxplots. No shading indicates an unassigned locomotor category in certain extant taxa (G. beringei, A. caraya, P. nemaeus). 


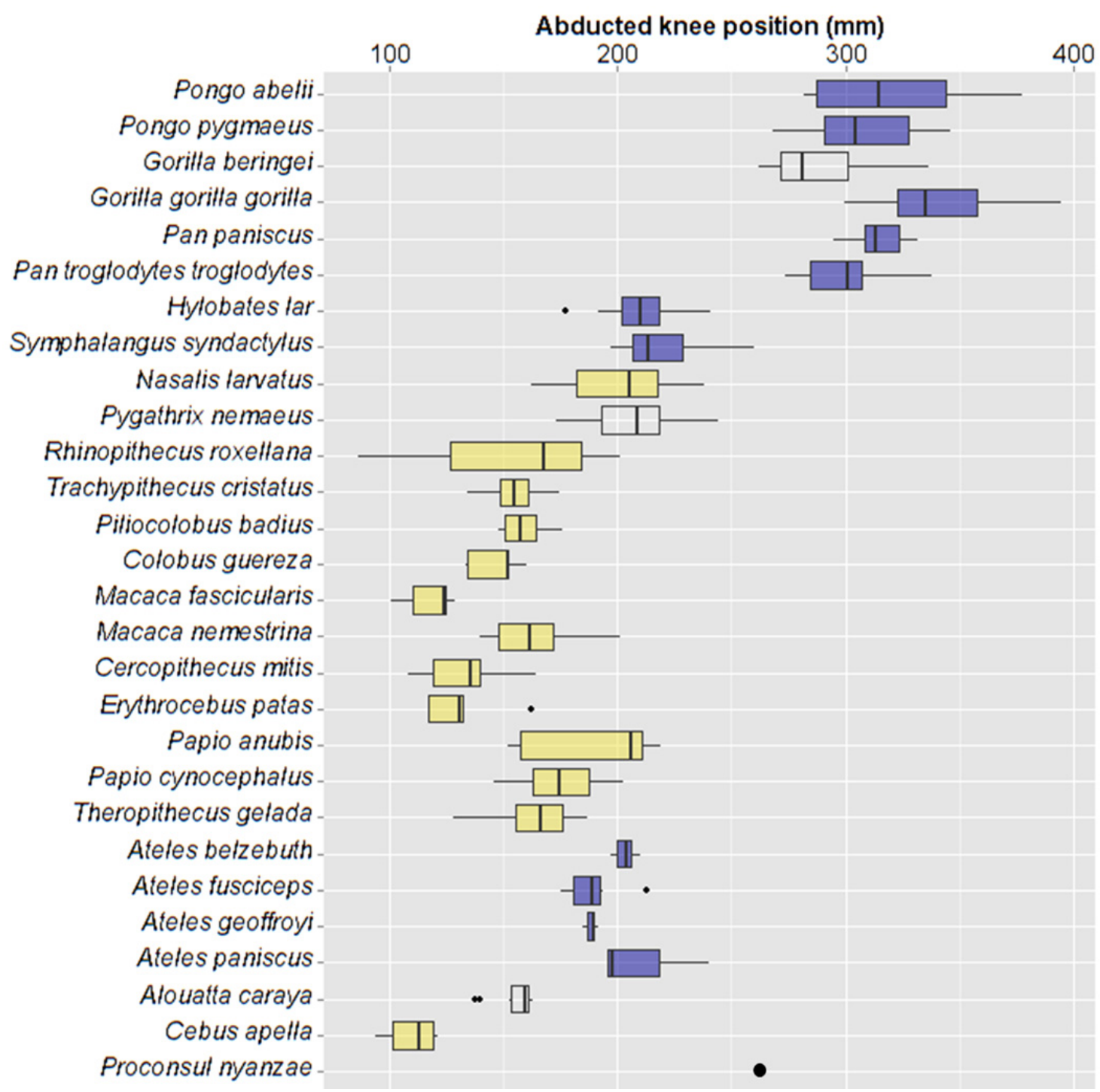

Figure 5.10. Abducted knee position of fossil specimens plotted alongside boxplots of extant taxa

Suspensory taxa are indicated by the darker blue shaded boxplots and non-suspensory taxa are indicated by yellow boxplots. No shading indicates an unassigned locomotor category in certain extant taxa (G. beringei, $A$. caraya, $P$. nemaeus). 


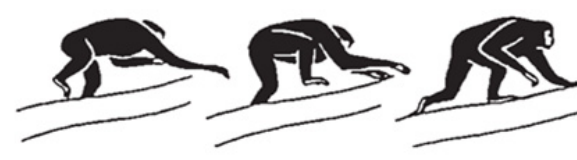

slow above-branch

quadrupedalism
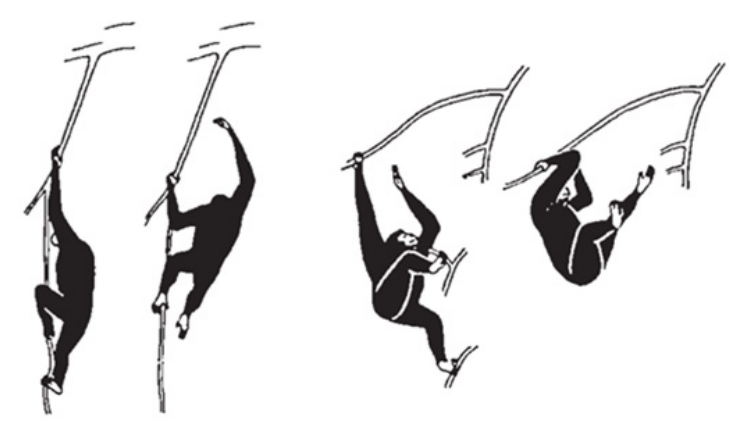

incorporation of more antipronograde climbing and transfer behaviors
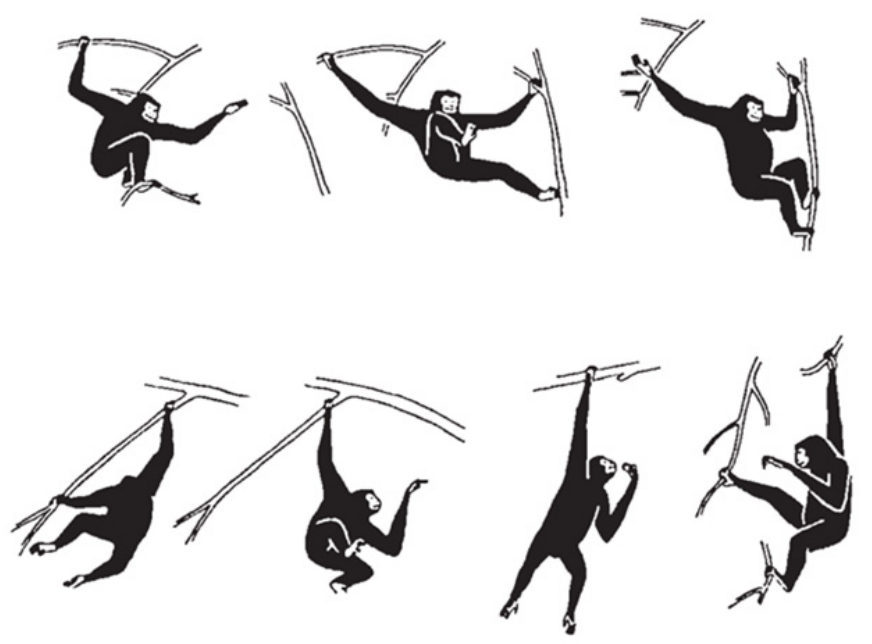

heavy reliance

on suspension

Figure 5.11 Hypothesized sequence of evolution of suspensory behaviors in Miocene hominoids

Above branch behaviors become increasingly antipronograde until eventually suspension becomes the primary mode of locomotion. Image modified from Nakatsukasa and Kunimatsu (2009), original illustrations from Fleagle (1976a). 


\section{CHAPTER 6: SUMMARY AND FUTURE DIRECTIONS}

The overarching goal of this dissertation is to address whether we can infer adaptations for suspensory behaviors in Miocene apes by modeling hip joint abduction. The results of two large studies were used to infer locomotor behaviors in fossil hominoids.

The first study tested was whether living suspensory and non-suspensory anthropoids differ in hip mobility, particularly in terms of abduction ability. Hominoids and atelines are known to use suspensory behaviors and have long been assumed to possess greater hip joint mobility than non-suspensory monkeys, an assumption that has greatly influenced how extant and fossil primate hip joint morphology has been interpreted, despite the fact that there are no data available on these taxa. Passive hip joint mobility was measured on a large sample of anesthetized captive anthropoids, and data were analyzed for significant differences by locomotor group (Chapter 3). The data demonstrate that suspensory anthropoids are capable of significantly greater hip abduction and external rotation. Degree of flexion and internal rotation were not larger in the suspensory primates, indicating that suspension is not associated with a global increase in hip mobility. Future work should consider the role of external rotation in abduction ability, how the physical position of the distal limb segments are influenced by differences in range of motion proximally, as well as focus on soft tissue differences that enable or restrict abduction and external rotation at the anthropoid hip joint. Excluding some published data for Macaca and Erythrocebus, the data presented in Chapter 3 represent the first known hip joint range of motion data for the anthropoids, and will 
prove to be important for other researchers who would like to compare other kinds of simulations to empirical data (e.g., Ogihara et al. 2009; Seth et al. 2011; O'Neill et al. 2013).

The second study developed a modeling technique to estimate range of hip abduction and then tested the accuracy of the modeling approach against the live animal data. Previous work by MacLatchy (MacLatchy 1995; 1996; MacLatchy and Bossert 1996; MacLatchy 1998) attempted to reconstruct anthropoid hip abduction by digitally articulating individual femora and matching pelves based on hypotheses about how synovial joints function under normal loading (Kapandji 1970; Jenkins 1972; Jenkins and Camazine 1977; Latimer et al. 1987; Ward 1991; 1993; Ward et al. 1993). Elaborating on MacLatchy's approach, hip joint abduction and the abducted knee position were reconstructed in a large sample of anthropoids (Chapter 4). These data were then compared to the in vivo data for passive range of abduction (Chapter 3 ) to see how well the models approximate empirical data.

The relationship between raw in vivo and in silico data for angle of abduction was shown to be predictable for most taxa. Abduction modeled in suspensory taxa in silico was slightly less than the passive range measured in vivo, as would be expected. Abduction modeled in non-suspensory taxa was usually slightly larger than the passive range. These results indicated that the modeling technique consistently conservatively estimated abduction in suspensory taxa but over-estimated abduction in non-suspensory taxa. Accordingly, the analyses provided a hypothetical framework for interpreting in silico abduction estimates for fossil taxa that fall at either ends of the spectrum of range of abduction. Only non-suspensory taxa had a range of abduction below $35^{\circ}$ and only suspensory taxa had values above $55^{\circ}$, but the in silico models showed substantial 
overlap in degrees of abduction between suspensory and non-suspensory taxa between $35^{\circ}$ and $55^{\circ}$ degrees. Although overlap between suspensory and non-suspensory taxa is not ideal for locomotor reconstructions in fossils, it is also not surprising given that different locomotor behaviors overlap in hindlimb postures. Even the most terrestrial monkeys will climb into trees to access foods or escape from predators, requiring basic arboreal competency and hip mobility in non-suspensory taxa such as Theropithecus and Papio. Modeling hip joint abduction is most useful in fossils that are found in the extreme ranges for the two locomotor groups (suspensory $>55^{\circ}$, non-suspensory $<35^{\circ}$ ), where adaptations for highly terrestrial or highly suspensory behaviors can be detected.

The relationship between raw in vivo and in silico data was shown to be especially predictable for abducted knee position. With the exception of Pan troglodytes, all in silico estimates of abducted knee position closely matched the in vivo measures. This indicates that the modeling approach usually converges on the functional outcome of hip mobility—-the abducted knee position—even when angular abduction is slightly over- or under-estimated by the models. However, unlike angular abduction, the abducted knee position has a significant relationship with body size. Although certain species will have similar abducted knee positions (e.g., macaque and siamang, or gorilla and orangutan), their angular abduction exposes significantly different levels of hip mobility and presumably different levels of hindlimb versatility. The abducted knee position is therefore most informative when considered along with angular abduction measures.

The validated model provides a way to make inferences about hip abduction ability and therefore potentially about locomotor behavior in Miocene apes. It was possible to model hip joint function in early hominoid Proconsul nyanzae (KNM-MW 
13142) using the same basic parameters as the extant sample. A modified approach that accommodated the preserved morphology was used in Rudapithecus hungaricus (undescribed pelvis and RUD 184 femur). Rudapithecus hungaricus had simulated abduction that was exclusively in the range of extant suspensory anthropoids, falling close to Ateles and hylobatids, but not within the range of Pongo. The results of the hip joint abduction simulations for Rudapithecus hungaricus are the first evidence for suspensory behavior in a Miocene ape based on joint function. Abduction simulations in Proconsul nyanzae yielded abduction similar to non-suspensory taxa and only within the range of a single suspensory species (Gorilla gorilla). When $R$. hungaricus and $P$. nyanzae are compared to each other, it is clear that $R$. hungaricus has a substantially larger range of abduction possible at its hip joint. Despite a somewhat limited range of abduction, however, simulations show that $P$. nyanzae would have been able to position its knee relatively far from the midline by virtue of being large.

This study has highlighted many aspects of hip function that remain to be explored. One of the most important next steps for assessing hip mobility and locomotor adaptation in Miocene hominoids involves identifying which hip morphologies actually relate to hip abduction ability. Most Miocene femora and pelves are incomplete, broken, or abraded, which limits our ability to estimate abduction ability by modeling. However, there are many fossil femora preserved for Miocene apes, including proximal femora from Hispanopithecus, Dryopithecus, Nacholapithecus, Morotopithecus, Equatorius, and Oreopithecus. If we understand which femoral morphologies can be used to predict abduction ability, we might be able to estimate hindlimb postures in a much broader sample of Miocene hominoids. This would allow us to chart the evolution of hip joint mobility in other taxa in addition to Proconsul and Rudapithecus, and extend inferences 
about locomotor evolution to different time periods and different taxa in hominoid evolution.

It would also be worthwhile to consider whether certain femoral and pelvic morphologies promote abduction ability per se or promote a combination of external rotation and abduction. The role of external (lateral) rotation in hominoid hip function has been vastly underappreciated. It is proposed here that external rotation facilitates the highly abducted hip postures observed in vivo in hominoids and atelines, and probably was influential in the in silico measures of abduction as well. Unfortunately, external rotation could not be accessed in the models in this study due to difficulties in quantifying external rotation in silico. Rose (1983) and MacLatchy and Bossert (1996) hypothesized that some features routinely found in Miocene hominoid femora, such as a high neck-shaft angle and even articular surface distribution, also relate to external rotation. The modeling approach used in this study can be further developed to test the importance of these morphologies, not only on abduction, but on external rotation as well.

Moving forward, it would be useful to examine how the configurations, composition and mechanical properties of soft tissues around the hip joint affect mobility. For instance, size of the acetabular labrum and strength of the hip joint ligaments should limit hip mobility but have not been thoroughly characterized in anthropoids. Preliminary studies note qualitative differences in the hip joint capsular structure between monkeys and great apes (Keith 1894; Sonntag 1923; 1924; Howell and Straus 1933), although it is unclear how these qualitative differences reflect mechanical differences of the capsular ligaments (see also Chapter 2). Gluteal and thigh muscular structure are also expected to be influential in both active and passive range of hip motion but this musculature is poorly characterized beyond general patterns of muscle origin and 
insertion in anthropoids (Sonntag 1923; 1924; Howell and Straus 1933; Raven 1950; Stern 1971; Diogo 2012). A few studies have quantified hindlimb muscle architecture, moment arms, and cross-sectional areas from just a few anthropoid taxa (Alexander 1981; Rauwerdink 1991; Thorpe et al. 1999; Payne 2001; Payne et al. 2006a; b), finding that suspensory anthropoids usually have muscular structure which should promote the production of force over a wider range of positions (Thorpe et al. 1999; Payne 2001; Payne et al. 2006a; b). However, it is still unclear how hip musculature itself limits hip mobility, much less how differences in structure or attachments in suspensory and nonsuspensory taxa influence range of motion. Just the presence of musculature around joints will significantly limit range of motion of the joint (see Hutson and Hutson 2012; Hutson and Hutson 2013). A slow and detailed dissection of the layers of soft tissues of the hip joint and thigh in anthropoids is necessary to understand how different soft tissues specifically limit hip mobility.

Additional work should focus on identifying how the femoral head is positioned in the acetabulum for different hip postures. Many of the principles guiding the joint modeling in this study are based on how hips are positioned during routine loading, and in particular, how the fovea capitis and ligamentum teres are positioned within the acetabulum (Kapandji 1970; Jenkins and Camazine 1977; Ward et al. 1993; Notzli et al. 2001). Functional morphologists hypothesize that routine loading of the ligamentum teres will result in joint pathologies and osteoarthritis, but no systematic experimental work has been done to test this. Clearly, the live animal work here (Chapter 3) suggests that chimpanzees are capable of passive abducted postures beyond what would be expected for their articular structure (Chapter 4), although it is unclear whether chimpanzees actively use these highly abducted hip postures. Additional study of how the femoral head is positioned in the acetabulum during loading is imperative and now 
possible with imaging technologies such as XROMM (e.g., Brainerd et al. 2010). Studies of in vivo joint configuration have applications beyond functional morphology. One such application is by incorporating the data in this thesis into models of how specific variation in human hip joint morphology affects hip mobility, which can be integrated into clinically relevant models of hip dysplasia, femoroacetabular impingement and joint congruence, and osteoarthritis.

Few field studies directly consider the role of hip postures during different anthropoid locomotor behaviors (Grand 1968; Fleagle 1976a), and additional field work characterizing the use of abduction and lateral thigh rotation in apes and atelines would be particularly useful for primate functional morphologists. With the exception of a single study on captive hominoid climbing (Isler 2003; 2005), there are no published kinematic data on hip abduction or external rotation in primates. Kinematic and behavioral data that quantitatively characterize hip postures during different locomotor behaviors will be integral to understanding how different hip abilities would have been selected for during the course of hominoid evolution. The hindlimb spatial envelope should also be investigated by behavioral and experimental researchers in order to have a gauge for how different ranges of foot postures reflect different substrate usage, environmental contexts, and different locomotor behaviors.

Testing the hypothesized link between hip morphology and abduction in other comparative taxa would serve to strengthen the functional inferences made in this study. With some additional work, this validated technique of measuring hip abduction can be made applicable to the study of any mammal. Limb abduction and posture (i.e., "sprawling" vs. "erect" postures) are also critical to models of locomotor evolution in early mammals (Kielan-Jaworowska and Hurum 2006), birds and reptiles (Gatesy 1991; Gatesy and Biewener 1991; Blob 2001; Farke and Alicea 2009; Hutchinson and Allen 
2009), and this project will serve as a comparative model for other researchers to approach joint function outside of anthropoid primates. Testing the relationship between hip morphology and abduction ability in a comparative sample outside of primates is necessary for testing the application of these findings to other taxa.

Determining how different Miocene taxa are related to each other and extant taxa is critical for testing virtually all hypotheses regarding ape and human evolution, including locomotor hypotheses (see Chapter 5). This work provides information about behavioral capacities of Miocene fossil apes, providing critical data for parsimony-based reconstructions of the primitive crown hominoid condition and hominin ancestors, information which is critical for evolutionary scenarios (Simons 1967; Tuttle 1975; MoyàSolà and Köhler 1993; Gebo 1996; Richmond and Strait 2000; Richmond et al. 2001; Thorpe et al. 2007b; Lovejoy et al. 2009b; Almécija et al. 2013). The postcrania of Ardipithecus ramidus has been used to argue that Pongo, Gorilla, and Pan independently evolved below-branch suspensory adaptations from a generalized ancestor (Lovejoy 2009; Lovejoy et al. 2009a; Lovejoy et al. 2009b), and some experimental evidence also suggests certain great ape-like adaptations independently evolved (Schmitt 2003; Kivell and Schmitt 2009). However, this study adds support to existing evidence for suspensory specializations in multiple European fossil apes thought to be crown hominoids, all of which predate the appearance of Ardipithecus (Moyà-Solà and Köhler 1993; 1996; Begun et al. 1997b; Moyà-Solà et al. 2004; Almécija et al. 2007; Begun 2010; Almécija et al. 2011; Begun and Kordos 2011). If the last common ancestor between Pan and humans was a primitive ape, not specialized for great ape-like locomotion, this would require that many fossil hominoids are homoplastic (review in Wood and Harrison 2011). Regardless of whichever phylogeny is ultimately 
supported, accurate reconstructions of hominoid locomotor evolution require further research into the behavioral capabilities and positional adaptations of fossil taxa. 


\section{BIBLIOGRAPHY}

Abel O. 1902. Zwei neue Menschenaffen aus den Leithakalk-bildungen des Wiener Beckens. Sitzungsberichte-Akademie Wissenschaften in Wien, mathematischnaturwissenschaftliche Klasse 1:1171-1207.

Abel O. 1931. Die Stellung des Menschen im Rahmen der Wirbeltiere. Jena: Gustav Fischer.

Aguilar MA, Aguilar FJ, and Negreiros J. 2009. Off-the-shelf laser scanning and closerange digital photogrammetry for measuring agricultural soils microrelief. Biosystems Engineering 103(4):504-517.

Aiello LC. 1992. Allometry and the analysis of size and shape in human evolution. J Hum Evol 22(2):127-147.

Alba DM. 2012. Fossil apes from the Vallès-Penedès Basin. Evol Anthropol 21:254-261.

Alba DM, Almécija S, Casanovas-Vilar I, Méndez JM, and Moyà-Solà S. 2012. A partial skeleton of the fossil great ape Hispanopithecus laietanus from Can Feu and the mosaic evolution of crown-hominoid positional behaviors. PLoS ONE 7(6):e39617.

Alba DM, Almécija S, and Moyà-Solà S. 2010a. Locomotor inferences in Pierolapithecus and Hispanopithecus: Reply to Deane and Begun (2008). J Hum Evol 59(1):143149.

Alba DM, Moyà-Solà S, and Almécija S. 2010b. A partial hominoid humerus from the middle miocene of Castell de Barbera (Valles-Penedes Basin, Catalonia, Spain). Am J Phys Anthropol.

Alexander RM. 1980. Forces in animal joints. Engineering in Medicine 9(2):93-97.

Alexander RM. 1985. Body support, scaling, and allometry. Functional vertebrate morphology:26-37.

Alexander RMN. 1981. Mechanics of skeleton and tendons. In: Brooks VB, editor. Handbook of physiology The nervous system. Bethesda: American Physiological Society.

Almécija S, Alba DM, and Moyà-Solà S. 2009. Pierolapithecus and the functional morphology of Miocene ape hand phalanges: paleobiological and evolutionary implications. J Hum Evol 57(3):284-297. 
Almécija S, Alba DM, and Moyà-Solà S. 2011. Large-hominoid remains from the Middle Miocene locality of Castell de Barbera (Valle`s-Penede`s Basin, Catalonia, Spain). Am J Phys Anthropol 144(S52):74-75.

Almécija S, Alba DM, Moyà-Solà S, and Köhler M. 2007. Orang-like manual adaptations in the fossil hominoid Hispanopithecus laietanus: first steps towards great ape suspensory behaviours. Proc R Soc B 274(1624):2375-2384.

Almécija S, Tallman M, Alba DM, Pina M, Moyà-Solà S, and Jungers WL. 2013. The femur of Orrorin tugenensis exhibits morphometric affinities with both Miocene apes and later hominins. Nat Commun 4:2888.

American Academy of Orthopedic Surgeons. 1965. Joint motion: method of measuring and recording. Chicago: The Association.

Anapol F, and Barry K. 1996. Fiber architecture of the extensors of the hindlimb in semiterrestrial and arboreal guenons. Am J Phys Anthropol 99(3):429-447.

Andrews P. 1982. Ecological polarity in primate evolution. Zoological Journal of the Linnean Society 74(3):233-244.

Andrews P, Harrison T, Delson E, Bernor RL, and Martin L. 1996. Distribution and biochronology of European and Southwest Asian Miocene catarrhines. The evolution of western eurasian neogene mammal faunas. New York: Columbia University Press. p 168-207.

Andrews P, and Simons E. 1977. A New African Miocene Gibbon-Like Genus, Dendropithecus (Hominoidea, Primates) with Distinctive Postcranial Adaptations: Its Significance to Origin of Hylobatidae. Folia Primatol 28(3):161-169.

Andrews PJ, and Walker A. 1976. The primate and other fauna from Fort Ternan, Kenya. In: Isaac GL, and McCown ER, editors. Human Origins: Louis Leakey and the East African Evidence. Menlo Park, CA: WA Benjamin. p 279-304.

Anemone RL. 1990. The VCL hypothesis revisited: patterns of femoral morphology among quadrupedal and saltatorial prosimian primates. Am J Phys Anthropol 83(3):373-393.

Ankel-Simons F, Fleagle JG, and Chatrath PS. 1998. Femoral anatomy of Aegyptopithecus zeuxis, an early oligocene anthropoid. Am J Phys Anthropol 106(4):413-424.

Arnold C, Matthews LJ, and Nunn CL. 2010. The 10kTrees Website: A New Online Resource for Primate Phylogeny. Evol Anthropol 19:114-118.

Asfaw B. 1985. Proximal femur articulation in Pliocene hominids. Am J Phys Anthropol 68(4):535-538. 
Avis V. 1962. Brachiation: the crucial issue for Man's ancestry. S J Anthropol 18(2):119148.

Bacon AM. 2001. La locomotion des primates du Miocene d'Afrique et d'Europe Centre National de la Recherche Scientifique.

Bardakos NV, and Villar RN. 2009. The ligamentum teres of the adult hip. J Bone Joint Surg $\mathrm{Br}$ 91(1):8-15.

Beard CK, Teaford MF, and Walker A. 1986. New wrist bones of Proconsul africanus and $P$. nyanzae from Rusinga Island, Kenya. Folia Primatol 47(2-3):97-118.

Begun DR. 1988. Catarrhine phalanges from the Late Miocene (Vallesian) of Rudabánya, Hungary. J Hum Evol 17(4):413-438.

Begun DR. 1992. Phyletic diversity and locomotion in primitive European hominids. Am J Phys Anthropol 87(3):311-340.

Begun DR. 1993. New catarrhine phalanges from Rudabánya (Northeastern Hungary) and the problem of parallelism and convergence in hominoid postcranial morphology. J Hum Evol 24(5):373-402.

Begun DR. 1994. Relations among the great apes and humans: New interpretations based on the fossil great ape Dryopithecus. Am J Phys Anthropol 37(S19):11-63.

Begun DR. 2000. Middle Miocene hominoid origins. Science 287(5462):2375.

Begun DR. 2002. European Hominoids. In: Hartwig, editor. The Primate Fossil Record. Cambridge: Cambridge University Press. p 339-368.

Begun DR. 2006. Revision of the Dryopithecini. J Vert Paleontol 26:40A.

Begun DR. 2007. Fossil record of Miocene hominoids. In: Henke W, and Tattersall I, editors. Handbook of paleoanthropology. Heidelberg: Springer Verlag. p 921-977.

Begun DR. 2010. Miocene hominids and the origins of the African apes and humans. Annu Rev Anthropol 39(1):67-84.

Begun DR, Geraads D, and Gulec E. 2003a. The Çandir hominoid locality: implications for the timing and pattern of hominoid dispersal events. CourierForschungsinstitut Senckenberg:251-265.

Begun DR, Güleç E, and Geraads D. 2003b. Dispersal patterns of Eurasian hominoids: implications from Turkey. Deinsea 10:23-39.

Begun DR, and Kordos L. 1993. Revision of Dryopithecus brancoi SCHLOSSER, 1901 based on the fossil hominoid material from Rudabánya. J Hum Evol 25(4):271285. 
Begun DR, and Kordos L. 2011. New postcrania of Rudapithecus hungaricus from Rudabánya (Hungary). Am J Phys Anthropol 144(S52):86.

Begun DR, Nargolwalla MC, and Kordos L. 2008. Revision of the Dryopithecinae: phylogenetic and palaeobiogeographic implications. Am J Phys Anthropol S44:66.

Begun DR, Nargolwalla MC, and Kordos L. 2012. European Miocene hominids and the origin of the African ape and human clade. Evol Anthropol 21(1):10-23.

Begun DR, Teaford MF, and Walker A. 1994. Comparative and functional anatomy of Proconsul phalanges from the Kaswanga Primate Site, Rusinga Island, Kenya. J Hum Evol 26(2):89-165.

Begun DR, and Ward CV. 2005. Comment on "Pierolapithecus catalaunicus, a new Middle Miocene great ape from Spain". Science 308(5719):203c.

Begun DR, Ward CV, and Rose MD. 1997a. Events in hominoid evolution. In: Begun DR, Ward CV, and Rose MD, editors. Function, Phylogeny, and Fossils. New York: Plenum Press. p 389-416.

Begun DR, Ward CV, and Rose MD. 1997b. Function, phylogeny, and fossils : Miocene hominoid evolution and adaptations. New York: Plenum Press. p 424.

Benefit BR. 1999. Victoriapithecus: The key to Old World monkey and catarrhine origins. Evol Anthropol 7(5):155-174.

Benefit BR, and McCrossin ML. 1995. Miocene hominoids and hominid origins. Annu Rev Anthropol 24:237-256.

Bernor RL, Kordos L, Rook L, Agusti J, Andrews P, Armour-Chelu M, Begun DR, Cameron DW, Damuth J, and Daxner-Höck G. 2004. Recent advances on multidisciplinary research at Rudabánya, Late Miocene (MN9), Hungary: a compendium. Palaeontogr Ital 89:3-36.

Bicca-Marques JC, and Calegaro-Marques C. 1995. Locomotion of black howlers in a habitat with discontinuous canopy. Folia Primatol (Basel) 64(1-2):55-61.

Biewener AA. 1983. Allometry of quadrupedal locomotion: the scaling of duty factor, bone curvature and limb orientation to body size. J Exp Biol 105:147-171.

Birchette MG. 1982. The postcranial skeleton of Paracolobus chemeroni Massachusetts: Harvard University. p 507.

Blob RW. 2001. Evolution of hindlimb posture in nonmammalian therapsids: biomechanical tests of paleontological hypotheses. Paleobiology 27(1):14-38. 
Böhme M, Aziz HA, Prieto J, Bachtadse V, and Schweigert G. 2011. Biomagnetostratigraphy and environment of the oldest Eurasian hominoid from the Early Miocene of Engelswies (Germany). J Hum Evol 61(3):332-339.

Boone DC, and Azen SP. 1979. Normal range of motion of joints in male subjects. J Bone Joint Surg Am 61(5):756-759.

Brainerd EL, Baier DB, Gatesy SM, Hedrick TL, Metzger KA, Gilbert SL, and Crisco JJ. 2010. X-ray reconstruction of moving morphology (XROMM): precision, accuracy and applications in comparative biomechanics research. Journal of Experimental Zoology Part A: Ecological Genetics and Physiology 313(5):262-279.

Bramblett CA. 1967. Pathology in the Darajani baboon. Am J Phys Anthropol 26(3):331340.

Brinckmann P, Frobin W, and Leivseth G. 2002a. Mechanical aspects of the hip joint. In: Brinckmann P, Frobin W, and Leivseth G, editors. Musculoskeletal Biomechanics. New York: Thieme. p 69-84.

Brinckmann P, Frobin W, and Leivseth G. 2002b. Musculoskeletal biomechanics. New York: Thieme Medical Publishers, Incorporated.

Byron CD, and Covert HH. 2004. Unexpected locomotor behaviour: brachiation by an Old World monkey (Pygathrix nemaeus) from Vietnam. J Zool 263(1):101-106.

Cailliet R. 1978. Soft Tissue Pain and Disability. Philadelphia, PA: FA Davis Co.

Campbell CJ. 2008. Spider monkeys : behavior, ecology and evolution of the genus Ateles. Cambridge, UK ; New York: Cambridge University Press. p 410.

Cannon $\mathrm{CH}$, and Leighton M. 1994. Comparative locomotor ecology of gibbons and macaques: selection of canopy elements for crossing gaps. Am J Phys Anthropol 93(4):505-524.

Cant JG. 1986. Locomotion and feeding postures of spider and howling monkeys: field study and evolutionary interpretation. Folia Primatol (Basel) 46(1):1-14.

Cant JG. 1987. Positional Behavior of Female Bornean Orangutans (Pongo pygmaeus). Am J Primatol 12(1):71-90.

Cant JG, Youlatos D, and Rose MD. 2001. Locomotor behavior of Lagothrix lagothricha and Ateles belzebuth in Yasuni National Park, Ecuador: general patterns and nonsuspensory modes. J Hum Evol 41(2):141-166.

Cant JGH, Youlatos D, and Rose MD. 2003. Suspensory locomotion of Lagothrix lagothricha and Ateles belzebuth in Yasuní National Park, Ecuador. J Hum Evol 44(6):685-699. 
Cartmill M. 1974. Pads and claws in arboreal locomotion. In: Jenkins FA, editor. Primate Locomotion. New York: Academic Press. p 45-83.

Cartmill M. 1985. Climbing. In: Hildebrand M, Bramble DM, Liem KF, and Wake DB, editors. Functional Vertebrate Morphology Cambridge: Belknap Press. p 73-88.

Cartmill M, and Milton K. 1977. The lorisiform wrist joint and the evolution of "brachiating" adaptations in the hominoidea. Am J Phys Anthropol 47(2):249-272.

Casanovas-Vilar I, Alba DM, Garcés M, Robles JM, and Moyà-Solà S. 2011. Updated chronology for the Miocene hominoid radiation in Western Eurasia. Proc Natl Acad Sci USA 108(14):5554-5559.

Cerezal L, Kassarjian A, Canga A, Dobado MC, Montero JA, Llopis E, Rolón A, and Pérez-Carro L. 2010. Anatomy, Biomechanics, Imaging, and Management of Ligamentum Teres Injuries. Radiographics 30(6):1637-1651.

Chan LK. 2007a. Glenohumeral mobility in primates. Folia Primatol (Basel) 78(1):1-18.

Chan LK. 2007b. Scapular position in primates. Folia Primatol (Basel) 78(1):19-35.

Chan LK. 2008. The range of passive arm circumduction in primates: do hominoids really have more mobile shoulders? Am J Phys Anthropol 136(3):265-277.

Chan YC, Roos C, Inoue-Murayama M, Inoue E, Shih CC, and Vigilant L. 2012. A comparative analysis of $Y$ chromosome and mtDNA phylogenies of the Hylobates gibbons. BMC Evol Biol 12:150.

Channon AJ, Günther MM, Crompton RH, and Vereecke EE. 2009. Mechanical constraints on the functional morphology of the gibbon hind limb. Journal of Anatomy 215(4):383-400.

Charbonnier C, Kolo FC, Duthon VB, Magnenat-Thalmann N, Becker CD, Hoffmeyer P, and Menetrey J. 2011. Assessment of congruence and impingement of the hip joint in professional ballet dancers: a motion capture study. Am J Sports Med 39(3):557-566.

Chen HH, Li AF, Li KC, Wu JJ, Chen TS, and Lee MC. 1996. Adaptations of ligamentum teres in ischemic necrosis of human femoral head. Clin Orthop Relat $\operatorname{Res}(328): 268-275$.

Chivers DJ. 1972. The siamang and the gibbon in the Malay peninsula. In: Rumbaugh DM, editor. The gibbon and the siamang. Basel: Karger. p 103-135.

Ciochon RL. 1993. Evolution of the cercopithecoid forelimb: phylogenetic and functional implications from morphometric analyses. Berkeley: University of California Press. 
Cole TM. 1971. Goniometry: the measurement of joint motion. In: Krusen FH, Kottke FJ, and Ellwood PM, editors. Handbook of Physical Medicine and Rehabilitation. 2nd ed. Philadelphia, PA: WB Saunders Co. p 40-44.

Conroy GC. 1987. Problems of body-weight estimation in fossil primates. Int J Primatol 8(2):115-137.

Cordy JM, and Ginesu S. 1994. Fiume Santo (Sassari, Sardaigne, Italie): Un nouveau gisement à oréopithèque(Oreopithecidae, Primates, Mammalia). C R Acad Sci Paris Sér II 318:697-704.

Crawford MJ, Dy CJ, Alexander JW, Thompson M, Schroder SJ, Vega CE, Patel RV, Miller AR, McCarthy JC, Lowe WR et al. . 2007. The 2007 Frank Stinchfield Award. The biomechanics of the hip labrum and the stability of the hip. Clin Orthop Relat Res 465:16-22.

Crelin ES. 1988. Ligament of the head of the femur in the orangutan and Indian elephant. Yale J Biol Med 61(5):383-388.

Crompton RH, Vereecke EE, and Thorpe SK. 2008. Locomotion and posture from the common hominoid ancestor to fully modern hominins, with special reference to the last common panin/hominin ancestor. J Anat 212(4):501-543.

D'Aout K, Vereecke E, Schoonaert K, De Clercq D, Van Elsacker L, and Aerts P. 2004. Locomotion in bonobos (Pan paniscus): differences and similarities between bipedal and quadrupedal terrestrial walking, and a comparison with other locomotor modes. J Anat 204(5):353-361.

D'Lima DD, Urquhart AG, Buehler KO, Walker RH, and Colwell CW, Jr. 2000. The effect of the orientation of the acetabular and femoral components on the range of motion of the hip at different head-neck ratios. J Bone Joint Surg Am 82(3):315321.

Dagosto M, and Schmid P. 1996. Proximal femoral anatomy of omomyiform primates. J Hum Evol 30(1):29-56.

Daniels L, and Wonhingham C. 1972. Mucle Testing: Techniques of Manual Examtnation. Philadelphia, PA: WB Saunders Co.

Davenport RK. 1967. The orang-utan in Sabah. Folia Primatol 5:247-263.

Deane AS. 2012. New evidence for canine dietary function in Afropithecus turkanensis. J Hum Evol 62(6):707-719.

Deane AS, and Begun DR. 2008. Broken fingers: retesting locomotor hypotheses for fossil hominoids using fragmentary proximal phalanges and high-resolution polynomial curve fitting (HR-PCF). J Hum Evol 55(4):691-701. 
Deane AS, and Begun DR. 2010. Pierolapithecus locomotor adaptations: a reply to Alba et al.'s comment on Deane and Begun (2008). J Hum Evol 59(1):150-154.

DeGusta D, and Milton K. 1998. Skeletal pathologies in a population of Alouatta palliata: behavioral, ecological, and evolutionary implications. Int J Primatol 19(3):615650.

Delson E. 1986. An anthropoid enigma: Historical introduction to the study of Oreopithecus bambolii. J Hum Evol 15(7):523-531.

Demange MK, Kakuda CMS, Pereira CAM, Sakaki MH, and Albuquerque RF. 2007. Influence of the femoral head ligament on hip mechanical function. Acta Ortop Bras 15(4):187-190.

Demes B, and Gunther MM. 1989. Biomechanics and allometric scaling in primate locomotion and morphology. Folia Primatol (Basel) 53(1-4):125-141.

DeRousseau CJ. 1988. Osteoarthritis in Rhesus monkeys and gibbons: a locomotor model of joint degeneration. New York: Karger.

DeRousseau CJ, Rawlins RG, and Denlinger JL. 1983. Aging in the musculoskeletal system of rhesus monkeys: I. Passive joint excursion. Am J Phys Anthropol 61(4):483-494.

Diogo R. 2012. Photographic and descriptive musculoskeletal atlas of gibbons and siamangs (Hylobates) : with notes on the attachments, variations, innervation, synonymy, and weight of the muscles. Boca Raton, FL: CRC Press. p 168 p.

Dodds MK, Lee J, and McCormack D. 2008. Transarticular stabilization of the immature femoral head: assessment of a novel surgical approach to the dislocating pediatric hip in a porcine model. J Pediatr Orthop 28(1):36-42.

Doran DM. 1993a. Comparative locomotor behavior of chimpanzees and bonobos: The influence of morphology on locomotion. Am J Phys Anthropol 91(1):83-98.

Doran DM. 1993b. Sex differences in adult chimpanzee positional behavior: the influence of body size on locomotion and posture. Am J Phys Anthropol 91(1):99115.

Doran DM. 1996. The comparative positional behaviour of the African apes. In: McGrew W, and Nishida T, editors. Great Ape Societies. Cambridge, UK: Cambridge University Press. p 213-224.

Doran DM. 1997. Ontogeny of locomotion in mountain gorillas and chimpanzees. J Hum Evol 32(4):323-344.

Doran DM, and Hunt KD. 1994. Comparative locomotor behavior of chimpanzees and bonobos: species and habitat differences. In: Wrangham RW, and McGrew WC, editors. Chimpanzee Cultures. Cambridge: Harvard University Press. p 93-106. 
Doran DM, and McNeilage A. 1998. Subspecific variation in gorilla behavior: the influence of ecological and social factors. In: Robbins MM, Sicotte P, and Stewart $\mathrm{KJ}$, editors. Mountain Gorillas, Three Decades of Research at Karisoke. Cambridge: Cambridge University Press. p 123-149.

Eck GG. 1987. Theropithecus oswaldi from the Shungura Formation, Lower Omo Basin, Southwestern Ethiopia. Les faunes Plio-Pleistocenes de la Basse Vallee de l'Omo (Ethiopie) Tome 3:123-141.

Ehrensberg K. 1938. Austriacopithecus, ein neuer menschen-affenartiger primate aus dem Miozan von klein-Hadersdorf bei Poysdorf in Niederosterreich (NiederDonau). Per Adad Wiss Wein, Math-nat kl, abstr 1:r71-100.

Eisenberg JF, and Kuehn RE. 1966. The behavior of Ateles geoffroyi and related species. Smithson Misc Coll 151:1-63.

Elton S. 2002. A reappraisal of the locomotion and habitat preference of Theropithecus oswaldi. Folia Primatol (Basel) 73(5):252-280.

Enstam KL, and Isbell LA. 2004. Microhabitat preference and vertical use of space by patas monkeys (Erythrocebus patas) in relation to predation risk and habitat structure. Folia Primatol (Basel) 75(2):70-84.

Erikson GE. 1963. Brachiation in New World monkeys and in anthropoid apes. In: Napier $\mathrm{J}$, and Barnicet NA, editors. The Primates. London: J Zool (Lond). p 135-163.

Ersoy A, Kelley J, Andrews P, and Alpagut B. 2008. Hominoid phalanges from the middle Miocene site of Paşalar, Turkey. J Hum Evol 54(4):518-529.

Esch D, and Lepley M. 1974. Evaluation of joint motion: methods of measurement and recording. Minneapolis: University of Minnesota Press.

Farke AA, and Alicea J. 2009. Femoral strength and posture in terrestrial birds and nonavian theropods. Anat Rec 292(9):1406-1411.

Feibel CS, and Brown FH. 1991. Age of the primate-bearing deposits on Maboko Island, Kenya. J Hum Evol 21(3):221-225.

Felsenstein J. 1985. Phylogenies and the comparative method. American Naturalist:115.

Finarelli JA, and Clyde WC. 2004. Reassessing hominoid phylogeny: evaluating congruence in the morphological and temporal data. Paleobiology 30(4):614-651.

Fleagle J. 1974. Dynamics of a brachiating siamang (Hylobates (Symphalangus) syndactylus). Nature 248(445):259-260. 
Fleagle J, Mittermeier R, and Skopec A. 1981a. Differential habitat use by Cebus apella and Saimiri sciureus in central Surinam. Primates 22(3):361-367.

Fleagle J, Susman RL, and Jungers WL. 1981b. Climbing: a biomechanical link with brachiation and with bipedalism. Symp Zool Soc Lond 48:359-375.

Fleagle JG. 1976a. Locomotion and posture of the Malayan siamang and implications for hominoid evolution. Folia Primatol 26(4):245-269.

Fleagle JG. 1976b. Locomotor behavior and skeletal anatomy of sympatric Malaysian leaf-monkeys (Presbytis obscura and Presbytis melalophos). Yearbook of Physical Anthropology 20:440-453.

Fleagle JG. 1977. Locomotor behavior and muscular anatomy of sympatric Malaysian leaf-monkeys (Presbytis obscura and Presbytis melalophos). Am J Phys Anthropol 46(2):297-307.

Fleagle JG. 1980. Locomotion and posture. In: Chivers DJ, editor. Malayan Forest Primates: Ten Year's Study in Tropical Rain Forest. New York: Plenum Press. $p$ 191-207.

Fleagle JG. 1983. Locomotor Adaptations of Oligocene and Miocene Hominoids and Their Phyletic Implications. In: Ciochon R, and Corruccini R, editors. New Interpretations of Ape and Human Ancestry: Springer US. p 301-324.

Fleagle JG. 1998. Primate Adaptation and Evolution. New York: Academic Press.

Fleagle JG. 2013. Primate Adaptation and Evolution, 3rd Ed.: Academic Press.

Fleagle JG, and Anapol FC. 1992. The indriid ischium and the hominid hip. J Hum Evol 1992(22):285-305.

Fleagle JG, and Meldrum DJ. 1988. Locomotor Behavior and Skeletal Morphology of Two Sympatric Pitheciine Monkeys, Pithecia pithecia and Chiropotes satanas. Am J Primatol 16:227-249.

Fleagle JG, and Mittermeier RA. 1980. Locomotor behavior, body size, and comparative ecology of seven Surinam monkeys. Am J Phys Anthropol 52(3):301-314.

Fleagle JG, and Simons EL. 1979. Anatomy of the bony pelvis in parapithecid primates. Folia Primatol (Basel) 31(3):176-186.

Fleagle JG, and Simons EL. 1995. Limb skeleton and locomotor adaptations of Apidium phiomense, an Oligocene anthropoid from Egypt. Am J Phys Anthropol 97(3):235-289.

Freckleton RP, Harvey PH, and Pagel M. 2002. Phylogenetic analysis and comparative data: a test and review of evidence. Am Nat 160(6):712-726. 
Friess M. 2012. Scratching the Surface? The use of surface scanning in physical and paleoanthropology. J Anthropol Sci.

Frost SR, and Delson E. 2002. Fossil Cercopithecidae from the Hadar Formation and surrounding areas of the Afar Depression, Ethiopia. J Hum Evol 43(5):687-748.

Frost SR, Marcus LF, Bookstein FL, Reddy DP, and Delson E. 2003. Cranial allometry, phylogeography, and systematics of large-bodied papionins (primates: Cercopithecinae) inferred from geometric morphometric analysis of landmark data. Anat Rec A Discov Mol Cell Evol Biol 275(2):1048-1072.

Furuya Y. 1961. The social life of silvered leaf monkeys. Primates 3(2):41-60.

Gajdosik RL. 2001. Passive extensibility of skeletal muscle: review of the literature with clinical implications. Clinical Biomechanics 16(2):87-101.

Garland Jr T, and Ives AR. 2000. Using the Past to Predict the Present: Confidence Intervals for Regression Equations in Phylogenetic Comparative Methods. Am Nat 155(3):346-364.

Garvin HM, and Ruff CB. 2012. Sexual dimorphism in skeletal browridge and chin morphologies determined using a new quantitative method. Am J Phys Anthropol 147(4):661-670.

Gatesy SM. 1991. Hind limb movements of the American alligator (Alligator mississippiensis) and postural grades. J Zool 224(4):577-588.

Gatesy SM, Baier DB, Jenkins FA, and Dial KP. 2010. Scientific rotoscoping: a morphology-based method of 3-D motion analysis and visualization. Journal of Experimental Zoology Part A: Ecological Genetics and Physiology 313(5):244261.

Gatesy SM, Baker M, and Hutchinson JR. 2009. Constraint-based exclusion of limb poses for reconstructing theropod dinosaur locomotion. Journal of Vertebrate Paleontology 29(2):535-544.

Gatesy SM, and Biewener AA. 1991. Bipedal locomotion: effects of speed, size and limb posture in birds and humans. J Zool 224(1):127-147.

Gebo DL. 1992. Locomotor and postural behavior in Alouatta palliata and Cebus capucinus. Am J Primatol 26(4):277-290.

Gebo DL. 1996. Climbing, brachiation, and terrestrial quadrupedalism: historical precursors of hominid bipedalism. Am J Phys Anthropol 101(1):55-92.

Gebo DL, and Chapman CA. 1995. Positional behavior in five sympatric old world monkeys. Am J Phys Anthropol 97(1):49-76. 
Gebo DL, MacLatchy LM, Kityo R, Deino AL, Kingston JD, and Pilbeam D. 1997. A Hominoid Genus from the Early Miocene of Uganda. Science 276(5311):401404.

Gerling ME, and Brown SH. 2013. Architectural analysis and predicted functional capability of the human latissimus dorsi muscle. J Anat 223(2):112-122.

Gervais P. 1872. Sur un singe fossile, d'espèce non encore décrite, qui a été découvert au Monte-Bamboli (Italie). CR Acad Sci Paris LXXIV:1217-1223.

Gibert J, Ribot F, Gibert L, Leakey M, Arribas A, and Martinez B. 1995. Presence of the Cercopithecid genus Theropithecus in Cueva Victoria (Murcia, Spain). J Hum Evol 28(5):487-493.

Gingerich PD. 1977. Correlation of tooth size and body size in living hominoid primates, with a note on relative brain size in Aegyptopithecus and Proconsul. Am J Phys Anthropol 47(3):395-398.

Gingerich PD, Smith BH, and Rosenberg K. 1982. Allometric scaling in the dentition of primates and prediction of body weight from tooth size in fossils. Am J Phys Anthropol 58(1):81-100.

Gittins SP. 1982. Feeding and ranging in the agile gibbon. Folia Primatol 38:39-71.

Godfrey L, Sutherland M, Boy D, and Gomberg N. 1991. Scaling of limb joint surface areas in anthropoid primates and other mammals. J Zool 223(4):603-625.

Godfrey LR. 1988. Adaptive diversification of Malagasy strepsirrhines. J Hum Evol 17(12):93-134.

Godfrey LR, Jungers WL, and Burney DA. 2010. Subfossil Lemurs of Madagascar. In: Werdelin L, and Sanders WJ, editors. Cenozoic mammals of Africa. Berkeley, CA: University of California Press. p 351-367.

Goldman CA, Snell RR, Thomason JJ, and Brown DB. 1990. Principles of Allometry. Tested Studies for Laboratory Teaching Conference of the Association for Biology Laboratory Education (ABLE), June 12-16, 1989. Fredericton, New Brunswick, Canada.

Goldsmith ML. 1999. Ecological Constraints on the Foraging Effort of Western Gorillas (Gorilla gorilla gorilla) at Bai Hoköu, Central African Republic. Int J Primatol 20(1):1-23.

Gommery D, Senut B, Pickford M, and Musiime E. 2002. Les nouveaux restes du squelette d'Ugandapithecus major (Miocène inférieur de Napak, Ouganda). Annales de Paléontologie 88(3):167-186.

Grafen A. 1989. The phylogenetic regression. Philos Trans R Soc Lond B Biol Sci 326(1233):119-157. 
Grand TI. 1968. The functional anatomy of the lower limb of the howler monkey (Alouatta caraya). Am J Phys Anthropol 28(2):163-181.

Grand TI. 1972. A Mechanical Interpretation of Terminal Branch Feeding. J Mammal 53(1):198-201.

Grand TI. 1984. Motion economy within the canopy: four strategies for mobility. In: Rodman PS, and Cant JGH, editors. Adaptations for foraging in nonhuman primates: contributions to an organismal biology of prosimians, monkeys, and apes. New York: Columbia University Press. p 52-72.

Guidi G, Remondino F, Morlando G, Del Mastio A, Uccheddu F, and Pelagotti A. 2008. Performances evaluation of a low cost active sensor for cultural heritage documentation. p 59-69.

Hall KRL. 1966. Behaviour and ecology of the wild Patas monkey, Erythrocebus patas, in Uganda. J Zool 148(1):15-87.

Hammond AS, Alba DM, Almécija S, and Moyá-Solá S. 2013a. Middle Miocene Pierolapithecus provides a first glimpse into early hominid pelvic morphology. $\mathrm{J}$ Hum Evol 64:658-666.

Hammond AS, Plavcan JM, and Ward CV. 2012. Precision and accuracy of acetabular size measures in fragmentary Plio-Pleistocene hominin pelves obtained using digital sphere-fitting techniques. Am J Phys Anthropol 147:159-160.

Hammond AS, Plavcan JM, and Ward CV. 2013b. Precision and accuracy of acetabular size measures in fragmentary Plio-Pleistocene hominin pelves obtained using digital sphere-fitting techniques. Am J Phys Anthropol 150:565-578.

Harding LE. 2010. Trachypithecus cristatus (Primates: Cercopithecidae). Mammalian Species:149-165.

Harmon EH. 2007. The shape of the hominoid proximal femur: a geometric morphometric analysis. Journal of Anatomy 210(2):170-185.

Harmon EH. 2009. The shape of the early hominin proximal femur. Am J Phys Anthropol 139(2):154-171.

Harris JM, Brown FH, and Leakey MG. 1988. Stratigraphy and paleontology of Pliocene and Pleistocene localities west of Lake Turkana, Kenya. Contributions in Science 399.

Harrison T. 1986. A reassessment of the phylogenetic relationships of Oreopithecus bambolii gervais. J Hum Evol 15(7):541-583.

Harrison T. 1987. The phylogenetic relationships of the early catarrhine primates: a review of the current evidence. J Hum Evol 16(1):41-80. 
Harrison T. 1989. New postcranial remains of Victoriapithecus from the middle Miocene of Kenya. J Hum Evol 18(1):3-54.

Harrison T. 1991. The implications of Oreopithecus bambolii for the origins of bipedalism. In: Coppens Y, and Senut B, editors. Origine(s) de la bipédie chez les hominidés. Paris: Editions du Centre National de la Recherche Scientifique. p 235-244.

Harrison T. 1992. A reassessment of the taxonomic and phylogenetic affinities of the fossil catarrhines from fort Ternan, Kenya. Primates 33(4):501-522.

Harrison T. 1993. Cladistic concepts and the species problem in hominoid evolution. In: Fleagle JG, and MacPhee RDE, editors. Species, Species Concepts, and Primate Evolution. New York: Plenum Press. p 345-371.

Harrison T. 2002. Late Oligocene to middle Miocene catarrhines from Afro-Arabia. In: Hartwig WC, editor. The primate fossil record: Cambridge University Press.

Harrison T. 2010. Dendropithecoidea, Proconsuloidea, and Hominoidea. Cenozoic Mammals of Africa University of California Press, Berkeley:429-469.

Harrison T, and Rook L. 1997. Enigmatic anthropoid or misunderstood ape? The phylogenetic status of Oreopithecus bambolii reconsidered. In: Begun DR, Ward CV, and Rose MD, editors. Function, Phylogeny, and Fossils: Miocene Hominoid Evolution and Adaptations. New York: Plenum Press. p 327-362.

Heizmann EPJ, and Begun DR. 2001. The oldest Eurasian hominoid. J Hum Evol 41(5):463-481.

Hickman CS. 1988. Analysis of Form and Function in Fossils. American Zoologist 28(2):775-793.

Hlusko LJ. 2006. A new large Pliocene colobine species (Mammalia: primates) from Asa Issie, Ethiopia. Geobios 39(1):57-69.

Hlusko LJ. 2007. A new Late Miocene species of Paracolobus and other cercopithecidae (Mammalia: Primates) fossil from Lemudong'o, Kenya. Kirtlandia 56:72-85.

Hogervorst T, Bouma HW, and de Vos J. 2009. Evolution of the hip and pelvis. Acta Orthop Suppl 80(336):1-39.

Holec P, and Emry RJ. 2003. Chapter 24: Another Molar of the Miocene Hominid Griphopithecus suessi from the Type Locality at Sandberg, Slovakia. Bulletin of the American Museum of Natural History:625-631.

Hoppenfeld S. 1976. Physical Examination of the Spine and Extremities. New York, NY: Appleton-Century-Crofts. 
Howell AB, and Straus WL. 1933. The muscular system. In: Hartman CG, and Straus WL, editors. The anatomy of the rhesus monkey (Macaca mulatta). New York: Hafner Publishing Co. p 89-175.

Hunt KD. 1991. Positional behavior in the hominoidea. Int J Primatol 12(2):95-118.

Hunt KD. 2004. The special demands of great ape locomotion and posture. In: Russon AE, and Begun DR, editors. Evolution of Thought : Evolutionary Origins of Great Ape Intelligence. Cambridge, UK: Cambridge University Press. p 172-189.

Hürzeler J. 1949. Neubeschreibung von Oreopithecus bambolii Gervais. Schweizerische Paläontologische Abhandlungen 66:3-20.

Hürzeler J. 1958. Oreopithecus bambolii Gervais, a preliminary report, Verh. naturf. Ges Basel 69:1-47.

Hutchinson J, and Allen V. 2009. The evolutionary continuum of limb function from early theropods to birds. Naturwissenschaften 96(4):423-448.

Hutchinson JR. 2004. Biomechanical modeling and sensitivity analysis of bipedal running ability. II. Extinct taxa. Journal of Morphology 262(1):441-461.

Hutchinson JR. 2011. On the inference of function from structure using biomechanical modelling and simulation of extinct organisms. Biol Lett.

Hutchinson JR, Anderson FC, Blemker SS, and Delp SC. 2005. Analysis of hindlimb muscle moment arms in Tyrannosaurus rex using a three-dimensional musculoskeletal computer model: implications for stance, gait, and speed. Paleobiology 31(4):676-701.

Hutchinson JR, and Garcia M. 2002. Tyrannosaurus was not a fast runner. Nature 415(6875):1018-1021.

Hutson JD, and Hutson KN. 2012. A test of the validity of range of motion studies of fossil archosaur elbow mobility using repeated-measures analysis and the extant phylogenetic bracket. J Exp Biol 215(Pt 12):2030-2038.

Hutson JD, and Hutson KN. 2013. Using the American alligator and a repeatedmeasures design to place constraints on in vivo shoulder joint range of motion in dinosaurs and other fossil archosaurs. J Exp Biol 216(Pt 2):275-284.

Isbell LA, Pruetz JD, Lewis M, and Young TP. 1998. Locomotor activity differences between sympatric patas monkeys (Erythrocebus patas) and vervet monkeys (Cercopithecus aethiops): implications for the evolution of long hindlimb length in Homo. Am J Phys Anthropol 105(2):199-207.

Ishida H, Kunimatsu Y, Nakatsukasa M, and Nakano Y. 1999. New Hominoid Genus from the Middle Miocene of Nachola, Kenya. Anthropol Sci 107(2):189-191. 
Ishida H, Kunimatsu Y, Takano T, Nakano Y, and Nakatsukasa M. 2004. Nacholapithecus skeleton from the Middle Miocene of Kenya. J Hum Evol 46(1):69-103.

Isler K. 2003. 3D-kinematics of vertical climbing in hominoids. Zurich: Universitat Zurich. p 212.

Isler K. 2005. 3D-kinematics of vertical climbing in hominoids. Am J Phys Anthropol 126(1):66-81.

Isler K, and Thorpe SKS. 2003. Gait parameters in vertical climbing of captive, rehabilitant and wild Sumatran orang-utans (Pongo pygmaeus abelii). Journal of Experimental Biology 206(22):4081-4096.

Iwamoto T. 1993. The ecology of Theropithecus gelada. In: Jablonski NJ, editor. Theropithecus: the rise and fall of a primate genus. Cambridge: Cambridge University Press. p 441-452.

Jenkins FA. 1972. Chimpanzee bipedalism: cineradiographic analysis and implications for the evolution of gait. Science 178(63):877-879.

Jenkins FA. 1974. Tree shrew locomotion and the origins of primate arborealism. In: Jenkins FA, editor. Primate Locomotion. New York: Academic Press. p 85-115.

Jenkins FA, and Camazine SM. 1977. Hip structure and locomotion in ambulatory and cursorial carnivores. J Zool 181(3):351-370.

Jolly C. 1972. The clasification and natural history of Theropithecus (Simopithecus) (Andrews, 1916), baboons of the African Plio-Pleistocene. Bulletin of the British Museum (Natural History), Geology 22:1-123.

Jones AL. 2008. The evolution of brachiation in ateline primates, ancestral character states and history. Am J Phys Anthropol 137(2):123-144.

Jungers WL. 1985. Body size and scaling of limb proportions in primates. In: Jungers WL, editor. Size and Scaling in Primate Biology. New York: Plenum Press. p 345381.

Jungers WL. 1987. Body size and morphometric affinities of the appendicular skeleton in Oreopithecus bambolii (IGF 11778). J Hum Evol 16(5):445-456.

Jungers WL. 1990. Problems and methods in reconstructing body size in fossil primates. In: Damuth J, and MacFadden BJ, editors. Body Size in Mammalian Paleobiology: Estimation and Biological Implications. New York: Cambridge University Press.

Jungers WL. 1991. Scaling of postcranial joint size in hominoid primates. Human Evolution 6:391-399. 
Jungers WL, and Stern JT, Jr. 1980. Telemetered electromyography of forelimb muscle chains in gibbons (Hylobates lar). Science 208(4444):617-619.

Jungers WL, and Susman RL. 1984. Body size and skeletal allometry in African apes. The pygmy chimpanzee: Springer. p 131-177.

Kapandji IA. 1970. The physiology of the joints: Lower limb: Churchill Livingstone. p 256.

Keith A. 1894. The Ligaments of the Catarrhine Monkeys, with References to corresponding Structures in Man. J Anat Physiol 28(Pt 2):149-168.

Keith A. 1923. Hunterian Lectures On Man's Posture: Its Evolution And Disorders. Given At The Royal College Of Surgeons Of England. The British Medical Journal 1(3250):624-626.

Kelley J. 1995. A functional interpretive framework for the early homioid postcranium. Am J Phys Anthropol 38 S20:125.

Kelley J. 1997. Paleobiological and phylogenetic significance of life history in Miocene hominoids. In: Begun DR, Ward CV, and Rose MD, editors. Function, Phylogeny, and Fossils: Miocene Hominoid Evolution and Adaptations. New York: Plenum Press. p 173-208.

Kendall HO, Kendall FP, and Wadswonh GE. 1971. Muscles: Testing and Function. Baltimore, MD: Williams \& Wilkins.

Kern JA. 1964. Observations on the habits of the proboscis monkey, Nasalis larvatus (Wurmb), made in the Brunei Bay Area, Borneo. Zoologica, NY 49:183-192.

Kern JA. 1965. The proboscis monkey. Animals 6:522-526.

Kielan-Jaworowska Z, and Hurum JH. 2006. Limb posture in early mammals: Sprawling or parasagittal. Acta Palaeontologica Polonica 51(3):393-406.

Kivell TL, and Begun DR. 2009. New primate carpal bones from Rudabánya (late Miocene, Hungary): taxonomic and functional implications. J Hum Evol 57(6):697-709.

Kivell TL, and Schmitt D. 2009. Independent evolution of knuckle-walking in African apes shows that humans did not evolve from a knuckle-walking ancestor. Proc Natl Acad Sci USA 106(34):14241-14246.

Klingenberg CP. 2011. MorphoJ: an integrated software package for geometric morphometrics. Mol Ecol Resour 11(2):353-357.

Koehl MAR. 1996. When does morphology matter? Annu Rev Ecol Syst 27:501-542.

Köhler M, Alba DM, Moyà Solà S, and MacLatchy LM. 2002. Taxonomic affinities of the Eppelsheim femur. Am J Phys Anthropol 119(4):297-304. 
Köhler M, and Moyà-Solà S. 1997. Ape-like or hominid-like? The positional behavior of Oreopithecus bambolii reconsidered. Proceedings of the National Academy of Sciences of the United States of America 94(21):11747.

Kordos L, and Begun DR. 2001. Primates from Rudabánya: allocation of specimens to individuals, sex and age categories. J Hum Evol 40(1):17-39.

Krentz HB. 1993. Postcranial anatomy of extant and extinct species of Theropithecus. In: Jablonski NJ, editor. Theropithecus: the rise and fall of a primate genus. Cambridge, UK: Cambridge University Press.

Kretzoi M. 1969. Geschichte der Primaten under der hominisation. Symp Biol Hung 9:311.

Kretzoi M. 1975. New ramapithecines and Pliopithecus from the lower Pliocene of Rudabánya in north-eastern Hungary.

Kubiak-Langer M, Tannast M, Murphy SB, Siebenrock KA, and Langlotz F. 2007. Range of motion in anterior femoroacetabular impingement. Clin Orthop Relat Res 458:117-124.

Kumar S, Sharma R, Gulati D, Dhammi IK, and Aggarwal AN. 2011. Normal range of motion of hip and ankle in Indian population. Acta Orthop Traumatol Turc 45(6):421-424.

Kummer B. 1965. Die Biomechanik der aufrechten Haltung. Mitt Naturforsch Ges 22:239-259.

Larson SG. 1993. Functional morphology of the shoulder in primates. In: Gebo DL, editor. Postcranial Adaptation in Nonhuman Primates. De Kalb, IL: Northern Illinois University Press. p 45-69.

Larson SG. 1998. Parallel evolution in the hominoid trunk and forelimb. Evol Anthropol 6(3):87-99.

Lartet E. 1856. Note sur un grand singe fossile qui se rattache au groupe des singes superieurs. CR Acad Sci Paris 43:219-223.

Latimer B, Ohman JC, and Lovejoy CO. 1987. Talocrural joint in African hominoids: implications for Australopithecus afarensis. Am J Phys Anthropol 74(2):155-175.

Lauder GV. 1995. On the inference of function from structure. In: Thomason J, editor. Functional morphology in vertebrate paleontology. New York: Cambridge University Press. p 1-18.

Lavin SR, Karasov WH, Ives AR, Middleton KM, and Garland T, Jr. 2008. Morphometrics of the avian small intestine compared with that of nonflying mammals: a phylogenetic approach. Physiol Biochem Zool 81(5):526-550. 
Lawler RR, and Stamps C. 2002. The relationship between tail use and positional behavior in Alouatta palliata. Primates 43(2):147-152.

Le Gros Clark WE. 1959. The Antecedents of Man. Edinburgh: Edinburgh University Press.

Le Gros Clark WE, and Leakey LSB. 1951. The Miocene Hominoidea of East Africa. Fossil Mammals of Africa, No 1. London: British Museum of Natural History.

Leakey MG. 1982. Extinct large colobines from the Plio-Pleistocene of Africa. Am J Phys Anthropol 58(2):153-172.

Leakey MG, Teaford MF, and Ward CV. 2003. Cercopithecidae from Lothagam. Lothagam: The Dawn of Humanity in Eastern Africa Columbia University Press, New York:201-248.

Leakey RE. 1969. New Cercopithecidae from the Chemeron Beds of Lake Baringo, Kenya. Fossil Vertebrates of Africa 1:53-69.

Leakey RE, and Leakey MG. 1986. A new Miocene hominoid from Kenya. Nature 324(6093):143-146.

Leakey RE, Leakey MG, and Walker AC. 1988. Morphology of Afropithecus turkanensis from Kenya. Am J Phys Anthropol 76(3):289-307.

Leakey REF, and Walker A. 1985. New higher primates from the early Miocene of Buluk, Kenya. Nature 318(6042):173-175.

Lee JC. 1990. Sources of Extraneous Variation in the Study of Meristic Characters: The Effect of Size and of Inter-Observer Variability. Systematic Zoology 39(1):31-39.

Leunig M, Beck M, Stauffer E, Hertel R, and Ganz R. 2000. Free nerve endings in the ligamentum capitis femoris. Acta Orthop Scand 71(5):452-454.

Levangie PK, and Norkin CC. 2005. Joint structure and function: a comprehensive analysis. Philadelphia, PA: F.A. Davis Co. p xix, 588 p.

Li Y. 2002. Postnatal development of pelvic sexual dimorphism in four anthropoid primates. Baltimore: The Johns Hopkins University.

Li Y. 2007. Terrestriality and tree stratum use in a group of Sichuan snub-nosed monkeys. Primates 48:197-207.

Lippold LK. 1998. Natural History of Douc Langurs. In: Jablonski NJ, editor. The Natural History of the Doucs and Snub-Nosed Monkeys. London: World Scientific. p 191206. 
Lovejoy CO. 1975. Biomechanical perspectives on the lower limb of early hominids. In: Tuttle RH, editor. Primate Functional Morphology and Evolution. Paris: Mouton.

Lovejoy CO. 2009. Reexamining human origins in light of Ardipithecus ramidus. Science 326(5949):74e71-78.

Lovejoy CO, Simpson SW, White TD, Asfaw B, and Suwa G. 2009a. Careful climbing in the Miocene: the forelimbs of Ardipithecus ramidus and humans are primitive. Science 326(5949):70e71-78.

Lovejoy CO, Suwa G, Simpson SW, Matternes JH, and White TD. 2009b. The great divides: Ardipithecus ramidus reveals the postcrania of our last common ancestors with African apes. Science 326(5949):100-106.

MacLatchy LM. 1995. A Three-dimensional Analysis of the Functional Morphology of the Primate Hip Joint: Harvard University.

MacLatchy LM. 1996. Another look at the australopithecine hip. J Hum Evol 31(5):455476.

MacLatchy LM. 1998. Reconstruction of hip joint function in extant and fossil primates. In: Strasser E, Fleagle J, Rosenberger A, and McHenry H, editors. Primate locomotion: recent advances. New York: Plenum Press. p 111-130.

MacLatchy LM. 2004. The oldest ape. Evol Anthropol 13:90-103.

MacLatchy LM, and Bossert WH. 1996. An analysis of the articular surface distribution of the femoral head and acetabulum in anthropoids, with implications for hip function in Miocene hominoids. J Hum Evol 31(5):425-453.

MacLatchy LM, Gebo D, Kityo R, and Pilbeam D. 2000. Postcranial functional morphology of Morotopithecus bishopi, with implications for the evolution of modern ape locomotion. J Hum Evol 39(2):159-183.

MacLatchy LM, and Kingston J. 2013. More of the femur of Morotopithecus. Society of Vertebrate Paleontology Abstract Book:168.

Madar SI, Rose MD, Kelley J, MacLatchy LM, and Pilbeam D. 2002. New Sivapithecus postcranial specimens from the Siwaliks of Pakistan. J Hum Evol 42(6):705.

Manduell KL, Morrogh-Bernard HC, and Thorpe SKS. 2011. Locomotor behavior of wild orangutans (Pongo pygmaeus wurmbii) in disturbed peat swamp forest, Sabangau, Central Kalimantan, Indonesia. Am J Phys Anthropol 145(3):348-359.

Marivaux L, Beard KC, Chaimanee Y, Jaeger JJ, Marandat B, Soe AN, Tun ST, and Kyaw AA. 2008. Proximal femoral anatomy of a sivaladapid primate from the late middle Eocene Pondaung formation (central Myanmar). Am J Phys Anthropol 137(3):263-273. 
Martin HD, Savage A, Braly BA, Palmer IJ, Beall DP, and Kelly B. 2008. The Function of the Hip Capsular Ligaments: A Quantitative Report. Arthroscopy 24(2):188-195.

Martin RL, Palmer I, and Martin HD. 2011. Ligamentum teres: a functional description and potential clinical relevance. Knee Surg Sports Traumatol Arthrosc 20:12091214.

Martins EP, Diniz-Filho JA, and Housworth EA. 2002. Adaptive constraints and the phylogenetic comparative method: a computer simulation test. Evolution 56(1):113.

Marzke MW, Tocheri MW, Steinberg B, Femiani JD, Reece SP, Linscheid RL, Orr CM, and Marzke RF. 2010. Comparative 3D quantitative analyses of trapeziometacarpal joint surface curvatures among living catarrhines and fossil hominins. Am J Phys Anthropol 141(1):38-51.

Matsudaira K, and Ishida T. 2010. Phylogenetic relationships and divergence dates of the whole mitochondrial genome sequences among three gibbon genera. Molecular Phylogenetics and Evolution 55(2):454-459.

McCrossin ML, and Benefit BR. 1992. Comparative assessment of the ischial morphology of Victoriapithecus macinnesi. Am J Phys Anthropol 87(3):277-290.

McCrossin ML, and Benefit BR. 1994. Maboko Island and the Evolutionary History of Old World Monkeys and Apes. In: Corruccini RS, and Ciochon RL, editors. Integrative Paths to the Past: Paleoanthropological Advances in Honor of F Clark Howell. Englewood Cliffs, NJ: Prentice Hall. p 95-122.

McCrossin ML, and Benefit BR. 1997. On the relationship and adaptations of Kenyapithecus, a large-bodied homioid from the Middle Miocene of Eastern Africa. In: Begun DR, Ward CV, and Rose MD, editors. Function, Phylogeny and Fossils: Miocene hominoid evolution and adaptations. New York: Plenum Press. p 241-267.

McGinnis PM. 2005. Biomechanics of Sport and Exercise. Champaign, IL: Human Kinetics Publishers.

McHenry HM, and Corruccini RS. 1983. The wrist of Proconsul africanus and the origin of hominoid postcranial adaptations. New Interpretations of Ape and Human Ancestry: Springer. p 353-367.

Mendel F. 1976. Postural and locomotor behavior of Alouatta palliata on various substrates. Folia Primatol (Basel) 26(1):36-53.

Mittermeier RA. 1978. Locomotion and posture in Ateles geoffroyi and Ateles paniscus. Folia Primatol (Basel) 30(3):161-193. 
Mittermeier RA, and Fleagle JG. 1976. The locomotor and postural repertoires of Ateles geoffroyi and Colobus guereza, and a reevaluation of the locomotor category semibrachiation. Am J Phys Anthropol 45(2):235-255.

Mohr T. 1989. Musculoskeletal analysis: the hip. In: Scully RM, and Barnes MR, editors. Physical Tberapy. Philadelphia, PA: JB Lippincott Co. p 369-380.

Morbeck ME. 1977. Positional behavior, selective use of habitat substrate and associated non-posiitonal behavior in free-ranging Colobus guereza (Ruppel, 1835). Primates 18(1):35-58.

Morbeck ME. 1983. Miocene hominoid discoveries from Rudabánya: implications from the postcranial skeleton. In: Ciochon RL, and Corruccini RS, editors. New Interpretations of Ape and Human Ancestry. New York: Plenum Press. p 369404.

Moreside JM, and McGill SM. 2011. Quantifying normal 3D hip ROM in healthy young adult males with clinical and laboratory tools: hip mobility restrictions appear to be plane-specific. Clin Biomech (Bristol, Avon) 26(8):824-829.

Morgan ME, Barry JC, and Pilbeam D. 2011. New middle Miocene hominoid partial innominate from the Siwalik sequence of Pakistan. Am J Phys Anthropol 144(S52):119.

Mow VC, Proctor CS, and Kelly MA. 1989. Biomechanics of articular cartilage. In: Nordin $\mathrm{M}$, and Frankel VH, editors. Basic Biomechanics of the Musculoskeletal System. Philadelphia: Lee and Febiger. p 60-101.

Moyà-Solà S, and Köhler M. 1993. Recent discoveries of Dryopithecus shed new light on evolution of great apes. Nature 365(6446):543.

Moyà-Solà S, and Köhler M. 1996. A Dryopithecus skeleton and the origins of great-ape locomotion. Nature 379(6561):156.

Moyà-Solà S, Köhler M, Alba DM, Casanovas-Vilar I, and Galindo J. 2004. Pierolapithecus catalaunicus, a new Middle Miocene great ape from Spain. Science 306(5700):1339-1344.

Moyà-Solà S, Köhler M, Alba DM, Casanovas-Vilar I, Galindo J, Robles JM, Cabrera L, Garcés M, Almécija S, and Beamud E. 2009. First partial face and upper dentition of the middle Miocene hominoid Dryopithecus fontani from Abocador de Can Mata (Valles-Penedes Basin, Catalonia, NE Spain): Taxonomic and phylogenetic implications. Am J Phys Anthropol 139(2):126-145.

Myatt JP, Crompton RH, and Thorpe SK. 2011. Hindlimb muscle architecture in nonhuman great apes and a comparison of methods for analysing inter-species variation. J Anat 219(2):150-166. 
Myers CA, Register BC, Lertwanich P, Ejnisman L, Pennington WW, Giphart JE, LaPrade RF, and Philippon MJ. 2011. Role of the acetabular labrum and the iliofemoral ligament in hip stability: an in vitro biplane fluoroscopy study. Am J Sports Med 39 Suppl:85S-91S.

Nagano A, Umberger BR, Marzke MW, and Gerritsen KGM. 2005.

Neuromusculoskeletal computer modeling and simulation of upright, straightlegged, bipedal locomotion of Australopithecus afarensis (A.L. 288-1). Am J Phys Anthropol 126(1):2-13.

Nakai M. 2003. Bone and Joint Disorders in Wild Japanese Macaques from Nagano Prefecture, Japan. Int J Primatol 24(1):179-195.

Nakatsukasa M, and Kunimatsu Y. 2009. Nacholapithecus and its importance for understanding hominoid evolution. Evol Anthropol 18:113-119.

Nakatsukasa M, Kunimatsu Y, Nakano Y, and Ishida H. 2007. Vertebral morphology of Nacholapithecus kerioi based on KNM-BG 35250. J Hum Evol 52(4):347-369.

Nakatsukasa M, Kunimatsu Y, Shimizu D, Nakano Y, Kikuchi Y, and Ishida H. 2012. Hind limb of the Nacholapithecus kerioi holotype and implications for its positional behavior. Anthropol Sci 120(3):235-250.

Nakatsukasa M, Mbua E, Sawada Y, Sakai T, Nakaya H, Yano W, and Kunimatsu Y. 2010. Earliest colobine skeletons from Nakali, Kenya. Am J Phys Anthropol 143(3):365-382.

Nakatsukasa M, Ward CV, Walker A, Teaford MF, Kunimatsu Y, and Ogihara N. 2004. Tail loss in Proconsul heseloni. J Hum Evol 46(6):777-784.

Napier J. 1963. Brachiation and brachiators. Symposia of the Zoological Society (London) 10:183-194.

Napier JR. 1964. The evolution of bipedal walking in the hominids. Arch Biol (Liege) 75:673-708

Napier JR. 1967. Evolutionary aspects of primate locomotion. Am J Phys Anthropol 27(3):333-341.

Napier JR, and Napier PH. 1967. A Handbook of Living Primates. New York: Academic Press.

Napier JR, and Walker AC. 1967. Vertical clinging and leaping - a newly recognized category of locomotor behaviour of primates. Folia Primatol 6(3-4):204-219.

NextEngine. 2012. NextEngine Tech Specs

[http://www.nextengine.com/assets/pdf/scanner-techspecs.pdf]. 
Norkin CC, and White DJ. 1995. Measurement of Joint Motion: A Guide to Goniometry. Philadelphia: F. A. Davis Company.

Notzli HP, Muller SM, and Ganz R. 2001. [The relationship between fovea capitis femoris and weight bearing area in the normal and dysplastic hip in adults: a radiologic study]. Z Orthop Ihre Grenzgeb 139(6):502-506.

Nunn CL. 2011. The Comparative Approach in Evolutionary Anthropology and Biology: University of Chicago Press.

O'Neill MC, Lee LF, Larson SG, Demes B, Stern JT, Jr., and Umberger BR. 2013. A three-dimensional musculoskeletal model of the chimpanzee (Pan troglodytes) pelvis and hind limb. J Exp Biol 216(Pt 19):3709-3723.

Ogihara N, Makishima H, Aoi S, Sugimoto Y, Tsuchiya K, and Nakatsukasa M. 2009. Development of an anatomically based whole-body musculoskeletal model of the Japanese macaque (Macaca fuscata). Am J Phys Anthropol 139(3):323-338.

Orme D, Freckleton R, Thomas G, Petzoldt T, Fritz S, Isaac N, and Pearse W. 2012. Caper: comparative analyses of phylogenetics and evolution in $\mathrm{R}$.

Pagel M. 1999. Inferring the historical patterns of biological evolution. Nature 401(6756):877-884.

Patel BA, Susman RL, Rossie JB, and Hill A. 2009. Terrestrial adaptations in the hands of Equatorius africanus revisited. J Hum Evol 57(6):763-772.

Pauwel F. 1980. Biomechanics of the Locomotor Apparatus. New York: Springer-Verlag.

Payne RC. 2001. Musculoskeletal adaptations for climbing in hominoids and their role as exaptations for the acquisition of bipedalism. Liverpool: The University of Liverpool. p 376.

Payne RC, Crompton RH, Isler K, Savage R, Vereecke EE, Gunther MM, Thorpe SK, and D'Aout K. 2006a. Morphological analysis of the hindlimb in apes and humans. I. Muscle architecture. J Anat 208(6):709-724.

Payne RC, Crompton RH, Isler K, Savage R, Vereecke EE, Gunther MM, Thorpe SK, and D'Aout K. 2006b. Morphological analysis of the hindlimb in apes and humans. II. Moment arms. J Anat 208(6):725-742.

Pedretti LW. 1996. Occupational therapy: Practice skills for physical dysfunction. St. Louis, MO: Mosby.

Pickford M. 1985. A new look at Kenyapithecus based on recent discoveries in Western Kenya. J Hum Evol 14(2):113-143.

Pickford M, Sawada Y, Tayama R, Matsuda Y, Itaya T, Hyodo H, and Senut B. 2006. Refinement of the age of the Middle Miocene Fort Ternan Beds, Western Kenya, 
and its implications for Old World biochronology. Comptes Rendus Geoscience 338(8):545-555.

Pierce SE, Clack JA, and Hutchinson JR. 2012. Three-dimensional limb joint mobility in the early tetrapod Ichthyostega. Nature advance online publication.

Pilbeam D, Rose MD, Barry JC, and Ibrahim Shah SM. 1990. New Sivapithecus humeri from Pakistan and the relationship of Sivapithecus and Pongo. Nature 348(6298):237.

Pilbeam DR, Rose MD, Badgley C, and Lipschutz B. 1980. Miocene hominoids from Pakistan. Postilla 181:1-94.

Pilbeam DR, and Simons EL. 1971. Humerus of Dryopithecus from Saint Gaudens, France. Nature 229(5284):406-407.

Pilbeam DR, and Young NM. 2001. Sivapithecus and hominoid evolution: some brief comments. In: de Bonis L, Koufos GD, and Andrews P, editors. Hominoid Evolution and Climatic Change in Europe. Cambridge, UK: Cambridge University Press. p 349-364.

Pina M. 2011. Morphological and Functional Study of the Femur of Dryopithecus fontani (Hominidae, Primates) from Abocador de Can Mata (els Hostalets de Pierola, NE Spain): Universitat Autònoma de Barcelona.

Pina M, Alba DM, Almécija S, Fortuny J, and Moyà-Solà S. 2012. Brief communication: Paleobiological inferences on the locomotor repertoire of extinct hominoids based on femoral neck cortical thickness: The fossil great ape Hispanopithecus laietanus as a test-case study. Am J Phys Anthropol 149(1):142-148.

Plavcan JM, Provaznik D, and Spring R. 2012. Estimating femoral head size in fragmentary fossils using 3-D surface scans. Am J Phys Anthropol 147:S238.

Polk JD, Williams SA, and Peterson JV. 2009. Body size and joint posture in primates. Am J Phys Anthropol 140(2):359-367.

Prates H, and Bicca-Marques JC. 2008. Age-Sex Analysis of Activity Budget, Diet, and Positional Behavior in Alouatta caraya in an Orchard Forest. Int J Primatol 29(3):703-715.

Preuschoft H. 1961. Muskeln and Gelenke der Hinterextremitat des Gorillas. Morphologisches Jahrbuch 101:432-540.

R Core Team. 2012. R: A language and environment for statistical computing. In: Computing RFfS, editor. Vienna, Austria.

Raaum RL, Sterner KN, Noviello CM, Stewart C, and Disotell TR. 2005. Catarrhine primate divergence dates estimated from complete mitochondrial genomes: concordance with fossil and nuclear DNA evidence. J Hum Evol 48(3):237-257. 
Rae TC. 1999. Mosaic evolution in the origin of the Hominoidea. Folia Primatol (Basel) 70(3):125-135.

Rafferty KL, Walker A, Ruff CB, Rose MD, and Andrews PJ. 1995. Postcranial estimates of body weight in Proconsul, with a note on a distal tibia of $P$. major from Napak, Uganda. Am J Phys Anthropol 97(4):391-402.

Ralphs JR, and Benjamin M. 1994. The joint capsule: structure, composition, ageing and disease. J Anat 184 ( Pt 3):503-509.

Rauwerdink IGP. 1991. Muscle fibre and tendon lengths in the distal limb segments of primates. Zeitschrift für Morphologie und Anthropologie:331-340.

Raven HC. 1950. Regional anatomy of the gorilla. In: Gregory WK, editor. The Anatomy of the Gorilla: The Studies of HC Raven. New York: Columbia University Press. $p$ 15-188.

Remis MJ. 1995. Effects of body size and social context on the arboreal activities of lowland gorillas in the Central African Republic. Am J Phys Anthropol 97(4):413433.

Remis MJ. 1998. The gorilla paradox: effects of habitat and body size on the positional behavior of lowland and mountain gorillas. In: Strasser E, Fleagle JG, Rosenberger A, and McHenry HM, editors. Primate Locomotion. New York: Plenum Press. p 95-106.

Reynolds V. 1965. Some Behavioral Comparisons between the Chimpanzee and the Mountain Gorilla in the Wild. American Anthropologist 67(3):691-706.

Richmond BG, Begun DR, and Strait DS. 2001. Origin of human bipedalism: The knuckle-walking hypothesis revisited. Yrbk Phys Anthropol 44:70-105.

Richmond BG, and Strait DS. 2000. Evidence that humans evolved from a knucklewalking ancestor. Nature 404(6776):382-385.

Richmond BG, Wright BW, Grosse I, Dechow PC, Ross CF, Spencer MA, and Strait DS. 2005. Finite element analysis in functional morphology. Anat Rec A Discov Mol Cell Evol Biol 283(2):259-274.

Roaas A, and Andersson GB. 1982. Normal range of motion of the hip, knee and ankle joints in male subjects, 30-40 years of age. Acta Orthop Scand 53(2):205-208.

Roach KE, and Miles TP. 1991. Normal hip and knee active range of motion: the relationship to age. Phys Ther 71(9):656-665.

Rodman PS. 1979. Skeletal differentiation of Macaca fascicularis and Macaca nemestrina in relation to arboreal and terrestrial quadrupedalism. Am J Phys Anthropol 51(1):51-62. 
Rohlf FJ. 2001. Comparative methods for the analysis of continuous variables: geometric interpretations. Evolution 55(11):2143-2160.

Rohlf FJ. 2006. A comment on phylogenetic correction. Evolution 60(7):1509-1515.

Rook L, Bondioli L, Köhler M, Moyà-Solà S, and Macchiarelli R. 1999. Oreopithecus was a bipedal ape after all: evidence from the iliac cancellous architecture. Proc Natl Acad Sci USA 96(15):8795-8799.

Rook L, Martínez-Navarro B, and Clark Howell F. 2004. Occurrence of Theropithecus $s p$. in the Late Villafranchian of Southern Italy and implication for Early Pleistocene "out of Africa" dispersals. J Hum Evol 47(4):267-277.

Rook L, Oms O, Benvenuti MG, and Papini M. 2011. Magnetostratigraphy of the Late Miocene Baccinello-Cinigiano basin (Tuscany, Italy) and the age of Oreopithecus bambolii faunal assemblages. Palaeogeography, Palaeoclimatology, Palaeoecology 305:286-294.

Rose MD. 1973a. Quadrupedalism in New and Old World monkeys. London: University of London.

Rose MD. 1973b. Quadrupedalism in primates. Primates 14(4):337-357.

Rose MD. 1977. Positional behavior of Olive baboons (Papio anubis) and its relationship to maintenance and social activities. Primates 18(1):59-116.

Rose MD. 1979. Positional behavior of natural populations: some quantitative results of a field study of Colobus guereza and Cercopithecus aethiops. In: Morbeck ME, Preuschoft $\mathrm{H}$, and Gomberg N, editors. Environment, behavior, and morphology: dynamic interactions in primates. New York: Gustav Fischer. p 75-93.

Rose MD. 1983. Miocene hominoid postcranial morphology: monkey-like, ape-like, neither, or both? In: Ciochon RL, and Corruccini RS, editors. New Interpretations of Ape and Human Ancestry. New York: Plenum Press. p 405-417.

Rose MD. 1997. Functional and phylogenetic features of the forelimb in Miocene hominoids. In: Begun DR, Ward CV, and Rose MD, editors. Function, Phylogeny and Fossils: Miocene Hominoid Evolution and Adaptation. New York: Plenum Press. p 79-100.

Rose MD, Leakey MG, Leakey REF, and Walker AC. 1992. Postcranial specimens of Simiolus enjiessi and other primitive catarrhines from the early Miocene of Lake Turkana, Kenya. J Hum Evol 22(3):171-237.

Rose MD, Nakano Y, and Ishida H. 1996. Kenyapithecus postcranial specimens from Nachola, Kenya. African study monographs Supplementary issue 24:3-56. 
Rosenberger AL, and Strier KB. 1989. Adaptive radiation of the ateline primates. J Hum Evol 18(7):717-750.

Rossie JB, Simons EL, Gauld SC, and Rasmussen DT. 2002. Paranasal sinus anatomy of Aegyptopithecus: implications for hominoid origins. Proc Natl Acad Sci 99(12):8454-8456.

Rothschild BM. 2005. Primate spondyloarthropathy. Curr Rheumatol Rep 7(3):173-181.

Rothschild BM, and Ruhli FJ. 2005. Etiology of reactive arthritis in Pan paniscus, $P$. troglodytes troglodytes, and $P$. troglodytes schweinfurthii. Am J Primatol 66(3):219-231.

Rothschild BM, and Woods RJ. 1989. Spondyloarthropathy in gorillas. Semin Arthritis Rheum 18(4):267-276.

Rothschild BM, and Woods RJ. 1992a. Erosive arthritis and spondyloarthropathy in old world primates. Am J Phys Anthropol 88(3):389-400.

Rothschild BM, and Woods RJ. 1992b. Spondyloarthropathy as an Old World phenomenon. Semin Arthritis Rheum 21(5):306-316.

Rothschild BM, and Woods RJ. 1996. Inflammatory arthritis in Pongo. J Med Primatol 25(6):414-418.

Rubin CT, and Lanyon LE. 1982. Limb mechanics as a function of speed and gait: a study of functional strains in the radius and tibia of horse and dog. Journal of Experimental Biology 101(1):187-211.

Rubin CT, and Lanyon LE. 1984. Dynamic strain similarity in vertebrates; an alternative to allometric limb bone scaling. Journal of Theoretical Biology 107(2):321-327.

Ruff CB. 1988. Hindlimb articular surface allometry in hominoidea and Macaca, with comparisons to diaphyseal scaling. J Hum Evol 17(7):687-714.

Ruff CB, Walker A, and Teaford M. 1989. Body mass, sexual dimorphism and femoral proportions of Proconsul from Rusinga and Mfangano Islands, Kenya. J Hum Evol 18:515-536.

Russo GA, and Shapiro LJ. 2013. Reevaluation of the lumbosacral region of Oreopithecus bambolii. J Hum Evol 65(3):253-265.

Safran MR, Lopomo N, Zaffagnini S, Signorelli C, Vaughn ZD, Lindsey DP, Gold G, Giordano G, and Marcacci M. 2012. In vitro analysis of peri-articular soft tissues passive constraining effect on hip kinematics and joint stability. Knee Surg Sports Traumatol Arthrosc 21:1655-1663. 
Sarban S, Baba F, Kocabey Y, Cengiz M, and Isikan UE. 2007. Free nerve endings and morphological features of the ligamentum capitis femoris in developmental dysplasia of the hip. J Pediatr Orthop B 16(5):351-356.

Sarmiento EE. 1987. The phylogenetic position of Oreopithecus and its significance in the origin of the Hominoidea. Am Mus Novitates 2881:1-44.

Sarmiento EE. 1995. Cautious climbing and folivory: a model of hominoid differentation. Human Evolution 10(4):289-321.

Satoh J. 1965. The M. Iliotrochantericus (M. Iliocapsulo Trochantericus) in Macaques. Okajimas folia anatomica Japonica 40:323-337.

Schaller GB. 1963. The Mountain Gorilla, Ecology and Behavior. Chicago: The University of Chicago Press.

Schlosser M. 1901. Die menschenahnlichen zahne aus dem Bohnerz der Schwabischen Alb. Zool Anz 24:261-271.

Schmidt M, and Krause C. 2011. Scapula movements and their contribution to threedimensional forelimb excursions in quadrupedal primates. In: D'Aout $\mathrm{K}$, and Vereecke E, editors. Primate Locomotion: Linking Field and Laboratory Research. New York: Springer.

Schmitt D. 2003. Insights into the evolution of human bipedalism from experimental studies of humans and other primates. Journal of experimental biology 206(9):1437-1448.

Schön MA. 1968. The Muscular system of the red howling monkey. Washington: Smithsonian institution press.

Schultz AH. 1944. Age changes and variability in gibbons. A Morphological study on a population sample of a man-like ape. Am J Phys Anthropol 2(1):1-129.

Schultz AH. 1950. The physical distinctions of man. Proceedings of the American Philosophical Society 94(5):428-449.

Schultz AH. 1960. Einige beobachtungen und maße am skelett von Oreopithecus im vergleich mit anderen catarrhinen primaten. Z Morphol Anthrop 50:136-149.

Schultz AH. 1961. Vertebral column and thorax. Primatologia 4:1-66.

Schultz AH. 1969. Observations on the acetabulum of primates. Folia Primatol 11(3):181-199.

Senut B, Nakatsukasa M, Kunimatsu Y, Nakano Y, Takano T, Tsujikawa H, Shimizu D, Kagaya M, and Ishida H. 2004. Preliminary analysis of Nacholapithecus scapula and clavicle from Nachola, Kenya. Primates 45(2):97-104. 
Senut B, Pickford M, Gommery D, and Kunimatsu Y. 2000. Un nouveau genre d'hominoïde du Miocène inférieur d'Afrique orientale: Ugandapithecus major (Le Gros Clark \& Leakey, 1950). CR Acad Sci Paris 331(3):227-233.

Seth A, Sherman M, Reinbolt JA, and Delp SL. 2011. OpenSim: a musculoskeletal modeling and simulation framework for in silico investigations and exchange. Procedia IUTAM 2:212-232.

Sherwood RJ, Ward S, Hill A, Duren DL, Brown B, and Downs W. 2002. Preliminary description of the Equatorius africanus partial skeleton (KNM-TH 28860) from Kipsaramon, Tugen Hills, Baringo District, Kenya. J Hum Evol 42(1-2):63-73.

Sholts SB, Flores L, Walker PL, and Wärmländer SKTS. 2011. Comparison of coordinate measurement precision of different landmark types on human crania using a 3D laser scanner and a 3D digitiser: Implications for applications of digital morphometrics. International Journal of Osteoarchaeology 21(5):535-543.

Sholts SB, Wärmländer SKTS, Flores LM, Miller KWP, and Walker PL. 2010. Variation in the Measurement of Cranial Volume and Surface Area Using 3D Laser Scanning Technology. Journal of Forensic Sciences 55(4):871-876.

Sigmon BA. 1969. The scansorius muscle in pongids. Primates 10(3):247-261.

Sigmon BA. 1974. A functional analysis of pongid hip and thigh musculature. J Hum Evol 3:161-185.

Simons EL. 1967. Fossil primates and the evolution of some primate locomotor systems. Am J Phys Anthropol 26:241-254.

Simons EL. 1969. Late Miocene Hominid from Fort Ternan, Kenya. Nature 221(5179):448-451.

Simons EL. 1995. Egyptian oligocene primates: A review. Am J Phys Anthropol 38(S21):199-238.

Singleton M. 2002. Patterns of cranial shape variation in the Papionini (Primates: Cercopithecinae). J Hum Evol 42(5):547-578.

Slizewski A, Friess M, and Semal P. 2010. Surface scanning of anthropological specimens: nominal-actual comparison with low cost laser scanner and high end fringe light projection surface scanning systems. Quartär 57:179-187.

Smith MV, Costic RS, Allaire R, Schilling PL, and Sekiya JK. 2012. A biomechanical analysis of the soft tissue and osseous constraints of the hip joint. Knee Surg Sports Traumatol Arthrosc.

Smith MV, Panchal HB, Ruberte Thiele RA, and Sekiya JK. 2011. Effect of acetabular labrum tears on hip stability and labral strain in a joint compression model. Am J Sports Med 39 Suppl:103S-110S. 
Smith RJ. 2009. Use and misuse of the reduced major axis for line-fitting. Am J Phys Anthropol 140(3):476-486.

Smith RJ, and Jungers WL. 1997. Body mass in comparative primatology. J Hum Evol 32(6):523-559.

Sonntag CF. 1923. On the anatomy, physiology and pathology of the chimpanzee. Proc Zool Soc Lond 22:323-429.

Sonntag CF. 1924. On the anatomy, physiology and pathology of the orang-outan. Proc Zool Soc Lond 24:349-450.

Srikosamatara S. 1984. Notes on the ecology and behavior of the hoolock gibbon. In: Preuschoft H, Chivers DJ, Brockelman WY, and Creel N, editors. The Lesser Apes. Edinburgh: Edinburgh University Press. p 242-257.

Standing S, and Gray H. 2008. Gray's anatomy : the anatomical basis of clinical practice. Edinburgh: Churchill Livingstone.

Steinberg N, Hershkovitz I, Peleg S, Dar G, Masharawi Y, Heim M, and Siev-Ner I. 2006. Range of joint movement in female dancers and nondancers aged 8 to 16 years: anatomical and clinical implications. Am J Sports Med 34(5):814-823.

Steppacher SD, Tannast M, Werlen S, and Siebenrock KA. 2008. Femoral morphology differs between deficient and excessive acetabular coverage. Clinical Orthopaedics and Related Research 466(4):782-790.

Stern JT. 1971. Functional myology of the hip and thigh of cebid monkeys and its implications for the evolution of erect posture. Bibliotheca Primatologica, No 14. Basel: Karger.

Stern JT, Jr., and Larson SG. 1993. Electromyographic study of the obturator muscles in non-human primates: implications for interpreting the obturator externus groove of the femur. J Hum Evol 24(5):403-427.

Stern JT, Jr., and Susman RL. 1983. The locomotor anatomy of Australopithecus afarensis. Am J Phys Anthropol 60(3):279-317.

Stern JT, and Oxnard CE. 1973. Primate Locomotion: Some links with evolution and morphology. In: Hofer $\mathrm{H}$, Schultz $\mathrm{AH}$, and Starck D, editors. Primatologia, Handbook of Primatology, Vol 4. Basel: Karger.

Stern JT, and Susman RL. 1981. Electromyography of the gluteal muscles in Hylobates, Pongo, and Pan: Implications for the evolution of hominid bipedality. Am J Phys Anthropol 55(2):153-166. 
Steultjens MPM, Dekker J, van Baar ME, Oostendorp RAB, and Bijlsma JWJ. 2000. Range of joint motion and disability in patients with osteoarthritis of the knee or hip. Rheumatology 39(9):955-961.

Stevens KA, and Parrish JM. 1999. Neck posture and feeding habits of two jurassic sauropod dinosaurs. Science 284(5415):798-800.

Stevens NJ, Seiffert ER, O'Connor PM, Roberts EM, Schmitz MD, Krause C, Gorscak E, Ngasala S, Hieronymus TL, and Temu J. 2013. Palaeontological evidence for an Oligocene divergence between Old World monkeys and apes. Nature 497(7451):611-614.

Stewart C, and Disotell TR. 1998. Primate evolution-in and out of Africa. Current Biology 8(16):R582-R588.

Straus WL. 1963. The classification of Oreopithecus. In: Washburn SL, editor. Classification and human evolution. Chicago: Aldine. p 146-177.

Struhsaker TT. 2010. The red colobus monkeys : variation in demography, behavior, and ecology of endangered species. Oxford ; New York: Oxford University Press. $p$ xxvi, 349 p., 316 p. of plates.

Susanna I, Alba DM, Almécija S, and Moyà-Solà S. 2010. The lumbar vertebrae of the Middle Miocene stem great ape Pierolapithecus catalaunicus (Primates: Hominidae). Am J Phys Anthropol 141 S50:S227.

Susman RL. 1984. The locomotor behavior of Pan paniscus in the Lomako forest. In: Susman RL, editor. The Pygmy Chimpanzee. New York: Plenum Press. p 369394.

Susman RL. 2004. Oreopithecus bambolii: an unlikely case of hominidlike grip capability in a Miocene ape. J Hum Evol 46(1):103.

Susman RL, Badrian NL, and Badrian AJ. 1980. Locomotor behavior of Pan paniscus in Zaire. Am J Phys Anthropol 53(1):69-80.

Szalay FS, and Delson E. 1979. Evolutionary History of the Primates. New York: Academic Press.

Tannast M, Siebenrock KA, and Anderson SE. 2007. Femoroacetabular impingement: radiographic diagnosis-what the radiologist should know. AJR Am J Roentgenol 188(6):1540-1552.

Thorpe SK, and Crompton RH. 2006. Orangutan positional behavior and the nature of arboreal locomotion in Hominoidea. Am J Phys Anthropol 131(3):384-401.

Thorpe SK, Crompton RH, and Alexander RM. 2007a. Orangutans use compliant branches to lower the energetic cost of locomotion. Biol Lett 3(3):253-256. 
Thorpe SK, Crompton RH, Gunther MM, Ker RF, and McNeill Alexander R. 1999. Dimensions and moment arms of the hind- and forelimb muscles of common chimpanzees (Pan troglodytes). Am J Phys Anthropol 110(2):179-199.

Thorpe SK, Holder R, and Crompton RH. 2009. Orangutans employ unique strategies to control branch flexibility. Proc Natl Acad Sci USA 106(31):12646-12651.

Thorpe SK, Holder RL, and Crompton RH. 2007b. Origin of human bipedalism as an adaptation for locomotion on flexible branches. Science 316(5829):1328-1331.

Tillmann B. 1978. A contribution to the functional morphology of articular surfaces. Norm Pathol Anat (Stuttg) 34:1-50.

Ting NCH. 2001. A functional analysis of the hip and thigh of Paracolobus chemeroni and Paracolobus mutiwa: University of Missouri-Columbia.

Tocheri MW. 2009. Laser Scanning: 3D Analysis of Biological Surfaces. In: Sensen CW, and Hallgrímsson B, editors. Advanced Imaging in Biology and Medicine: Springer Berlin Heidelberg. p 85-101.

Tocheri MW, Razdan A, Williams RC, and Marzke MW. 2005. A 3D quantitative comparison of trapezium and trapezoid relative articular and nonarticular surface areas in modern humans and great apes. J Hum Evol 49(5):570-586.

Tocheri MW, Solhan CR, Orr CM, Femiani J, Frohlich B, Groves CP, Harcourt-Smith WE, Richmond BG, Shoelson B, and Jungers WL. 2011. Ecological divergence and medial cuneiform morphology in gorillas. J Hum Evol 60(2):171-184.

Tolhurst DE, and Hart J. 1990. Cadaver preservation and dissection. European Journal of Plastic Surgery 13(2):75-78.

Turnquist JE. 1983. Influence of age, sex, and caging on joint mobility in the Patas monkey. Am J Phys Anthropol 61(211-220).

Turnquist JE. 1985. Passive joint mobility in Patas monkeys (Erythrocebus patas): rehabilitation of caged animals after release into a free-ranging environment. Am J Phys Anthropol 67:1-5.

Turnquist JE, and Kessler MJ. 1989. Free-ranging Cayo Santiago Rhesus monkeys (Macaca mulatta): II. passive joint mobility. Am J Phys Anthropol 19:15-23.

Tuttle RH. 1975. Parallelism, brachiation and hominoid phylogeny. In: Luckett WP, and Szalay FS, editors. The Phylogeny of the Primates: A Multidisciplinary Approach. New York: Plenum Press. p 447-480.

Van Valkenburgh B. 1994. Ecomorphological analysis of fossil vertebrates and their paleocommunities. In: Wainwright PC, and Reilly SM, editors. Ecological morphology: integrative organismal biology. Chicago: University of Chicago Press. p 140-166. 
Venables WN, and Ripley BD. 2002. Modern applied statistics with S. New York: Springer.

Vereecke EE, D'Aout K, and Aerts P. 2006. Locomotor versatility in the white-handed gibbon (Hylobates lar): a spatiotemporal analysis of the bipedal, tripedal, and quadrupedal gaits. J Hum Evol 50(5):552-567.

Vereecke EE, D'Août K, and Aerts P. 2011. Studying Captive Ape Locomotion: Past, Present, and Future. In: D'Août K, and Vereecke EE, editors. Primate Locomotion: Springer New York. p 29-46.

Viidik A, and Lewin T. 1966. Changes in tensile strength characteristics and histology of rabbit ligaments induced by different modes of postmortal storage. Acta Orthop Scand 37(2):141-155.

Villalta Comella JF, and Crusafont Pairó M. 1944. Dos nuevos antropomorfos del Mioceno español, y su situación dentro de la moderna sistemática de los símidos. Not Com Inst Geol Min Esp 13:1-51.

von Klaus Uhlmann K. 1968. Hüft- und Oberschenkelmuskulatur. Systematische und vergleichende Anatomie. In: Hofer $\mathrm{H}$, Schultz $\mathrm{AH}$, and Stark D, editors.

Primatologia. Basel: Karger.

Wagner FV, Negrao JR, Campos J, Ward SR, Haghighi P, Trudell DJ, and Resnick D. 2012. Capsular ligaments of the hip: anatomic, histologic, and positional study in cadaveric specimens with MR arthrography. Radiology 263(1):189-198.

Walji AH. 1988. Some histological aspects of the hip joint capsule in the vervet monkey. J Morphol 197(3):327-335.

Walker A, Falk D, Smith R, and Pickford M. 1983. The skull of Proconsul africanus: reconstruction and cranial capacity.

Walker AC. 1974. Locomotor adaptations in past and present prosimian primates. In: Jenkins FA, editor. Primate Locomotion. New York: Academic Press. p 349-381.

Walker AC, and Pickford M. 1983. New postcranial fossils of Proconsul africanus and Proconsul nyanzae. New interpretations of ape and human ancestry: Springer. $p$ 325-351.

Wang W, Crompton RH, Carey TS, Günther MM, Li Y, Savage R, and Sellers WI. 2004. Comparison of inverse-dynamics musculo-skeletal models of AL 288-1 Australopithecus afarensis and KNM-WT 15000 Homo ergaster to modern humans, with implications for the evolution of bipedalism. J Hum Evol 47(6):453478. 
Ward CV. 1991. Functional Anatomy of the Lower Back and Pelvis of the Miocene Hominoid Proconsul nyanzae from the Miocene of Mfangano Island, Kenya Baltimore: The Johns Hopkins University.

Ward CV. 1992. Hip joints of Proconsul nyanzae and P. africanus. Am J Phys Anthropol S14:171.

Ward CV. 1993. Torso morphology and locomotion in Proconsul nyanzae. Am J Phys Anthropol 92(3):291-328.

Ward CV. 1998. Afropithecus, Proconsul, and the Primitive Hominoid Skeleton. In: Strasser E, Fleagle J, Rosenberger A, and McHenry H, editors. Primate Locomotion: Springer US. p 337-352.

Ward CV. 2007. Postcranial and locomotor adaptations of hominoids. In: Henke W, and Tattersall I, editors. Handbook of Paleoanthropology. Heidelberg: Springer Verlag. p 1011-1030.

Ward CV. 2009. Three dimensional anatomy of the anthropoid bony pelvis. Am J Phys Anthropol 138:S266-267.

Ward CV. 2013. The evolution of human anatomy and locomotion. American Association of Anatomists 2013 Annual Conference Proceedings:8.

Ward CV, Begun DR, and Kordos L. 2008. New partial pelvis of Dryopithecus brancoi from Rudabánya, Hungary. Am J Phys Anthropol 135(S46):218.

Ward CV, Hammond AS, Plavcan JM, Begun DR, and Kordos L. 2010. Hip joint morphology and hominoid evolution: From quadrupedal to suspensory to bipedal locomotion. Society of Vertebrate Paleontology, SVP Program and Abstracts Book:184A.

Ward CV, Walker A, and Teaford MF. 1991. Proconsul did not have a tail. J Hum Evol 21(3):215-220.

Ward CV, Walker A, Teaford MF, and Odhiambo I. 1993. Partial skeleton of Proconsul nyanzae from Mfangano Island, Kenya. Am J Phys Anthropol 90(1):77-111.

Ward SC. 1997. The Taxonomy and Phylogenetic Relationships of Sivapithecus Revisited. In: Begun D, Ward C, and Rose MD, editors. Function, Phylogeny, and Fossils: Miocene Hominoid Evolution and Adaptations. New York: Plenum Press. p 269-290.

Ward SC, Brown B, Hill A, Kelley J, and Downs W. 1999. Equatorius: a new hominoid genus from the Middle Miocene of Kenya. Science 285(5432):1382-1386.

Ward SC, and Duren DL. 2002. Middle and late Miocene African hominoids. In: Hartwig WC, editor. The Primate Fossil Record. Cambridge: Cambridge University Press. 
Ward SC, and Kimbel WH. 1983. Subnasal alveolar morphology and the systematic position of Sivapithecus. Am J Phys Anthropol 61(2):157-171.

Ward SC, and Pilbeam D. 1983. Maxillofacial morphology of Miocene hominoids from Africa and Indo-Pakistan. In: Ciochon RL, and Corruccini RS, editors. New Interpretations of Ape and Human Ancestry. New York: Plenum Press. p 325351.

Warton DI, Duursma RA, Falster DS, and Taskinen S. 2012. smatr 3- an R package for estimation and inference about allometric lines. Methods Ecol Evol 3(2):257-259.

Warton DI, Wright IJ, Falster DS, and Westoby M. 2006. Bivariate line-fitting methods for allometry. Biol Rev Camb Philos Soc 81(2):259-291.

Waterman HC. 1929. Studies on the evolution of the pelves of man and other primates. Am Mus Nat Hist Bull 58:585-642.

Wenger D, Miyanji F, Mahar A, and Oka R. 2007. The mechanical properties of the ligamentum teres: a pilot study to assess its potential for improving stability in children's hip surgery. J Pediatr Orthop 27(4):408-410.

Wilke HJ, Krischak S, and Claes LE. 1996. Formalin fixation strongly influences biomechanical properties of the spine. J Biomech 29(12):1629-1631.

Wood B, and Harrison T. 2011. The evolutionary context of the first hominins. Nature 470(7334):347-352.

Workman C, and Covert HH. 2005. Learning the ropes: The ontogeny of locomotion in red-shanked douc (Pygathrix nemaeus), Delacour's (Trachypithecus delacouri), and Hatinh langurs (Trachypithecus hatinhensis) I. positional behavior. Am J Phys Anthropol 128(2):371-380.

Wright KA. 2007. The relationship between locomotor behavior and limb morphology in brown (Cebus apella) and weeper (Cebus olivaceus) capuchins. Am J Primatol 69(7):736-756.

Wright KA, Stevens NJ, Covert HH, and Nadler T. 2008. Comparisons of suspensory behaviors among Pygathrix cinerea, $P$. nemaeus, and Nomascus leucogenys in Cuc Phuong National Park, Vietnam. Heidelberg, Germany: Springer. p 14.

Wright V, and Radin EL. 1993. Mechanics of Human Joints. New York: Marcel Dekker.

Wu BQ. 1993. Patterns of Spatial Dispersion, Locomotion and Foraging Behaviour in Three Groups of the Yunnan Snub-Nosed Langur (Rhinopithecus bieti). Folia Primatol 60(1-2):63-71.

Yamagiwa J, Maruhashi T, Yumoto T, and Mwanza N. 1998. Dietary and ranging overlap in sympatric gorillas and chimpanzees in Kahuzi-Biega National Park, 
Zaire. In: McGrew W, Marchant LF, and Nishida T, editors. Great Ape Societies. Cambridge: Cambridge University Press. p 82-98.

Ybarra MAS. 1984. Locomotion and postures of red howlers in a deciduous forestsavanna interface. Am J Phys Anthropol 63(1):65-76.

Yezerinac SM, Lougheed SC, and Handford P. 1992. Measurement error and morphometric studies: statistical power and observer experience. Cary, NC: Oxford University Press.

Youlatos D. 2002. Positional Behavior of Black Spider Monkeys (Ateles paniscus) in French Guiana. Int J Primatol 23(5):1071-1093.

Young NM. 2003. A reassessment of living hominoid postcranial variability: implications for ape evolution. J Hum Evol 45(6):441-464.

Young NM, and MacLatchy LM. 2004. The phylogenetic position of Morotopithecus. J Hum Evol 46(2):163.

Zihlman AL, McFarland RK, and Underwood CE. 2011. Functional anatomy and adaptation of male gorillas (Gorilla gorilla gorilla) with comparison to male orangutans (Pongo pygmaeus). Anatomical Record 294(11):1842-1855. 


\section{VITA}

Ashley Hammond attended Florida Atlantic University for her Bachelors and Masters degrees in Anthropology, discovering a strong interest in morphology and gross anatomy along the way. She moved to the University of Missouri's Department of Pathology and Anatomical Sciences for doctoral work. Her time in graduate school has allowed her to work in North America, Europe and Africa with many remarkable people, and these experiences have come to define her and her early career. 


\section{APPENDIX A}

PHYLOGENETIC TREE WITH BRANCH LENGTHS USED IN PHYLOGENETIC REGRESSIONS 


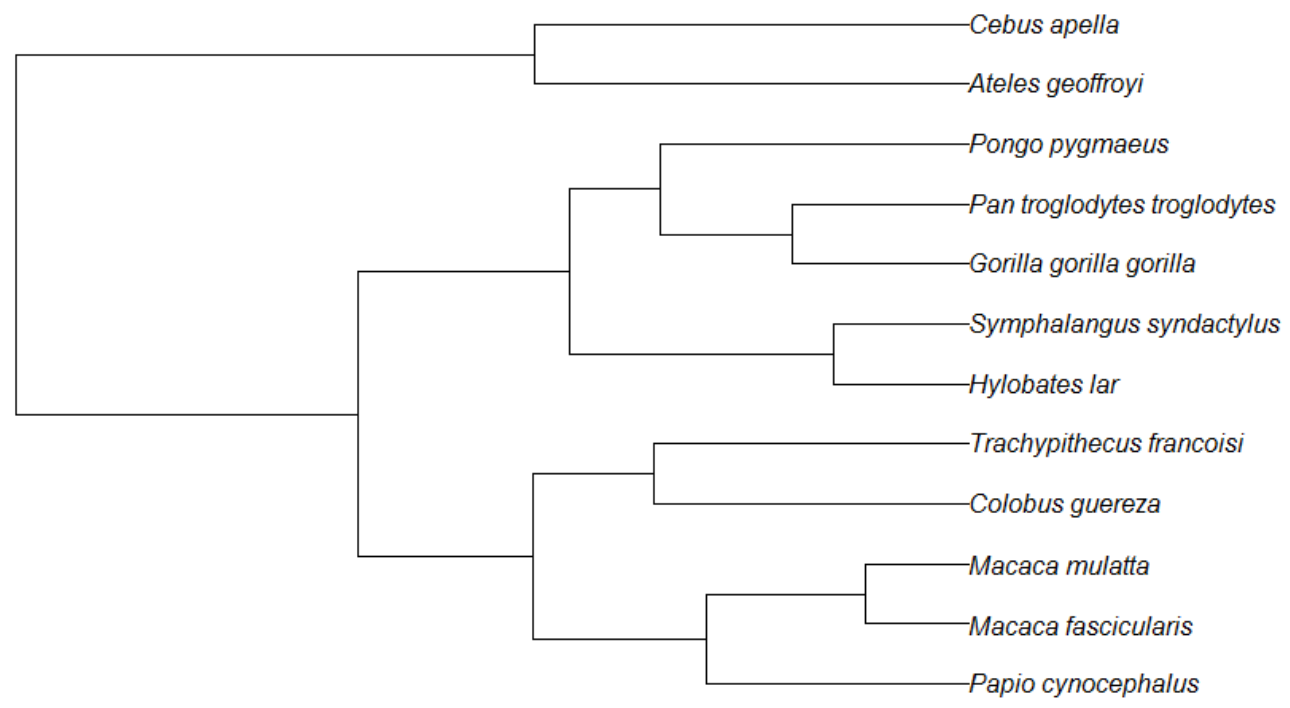

Consensus tree branch lengths:

(((Papio_cynocephalus:12.852525,(Macaca_fascicularis:5.045978,

Macaca_mulatta:5.045978):7.806547):8.557849,(Colobus_guereza:15.435157,

Trachypithecus_francoisi:15.435159):5.975217):8.589626,(( Hylobates_lar:6.598361, Symphalangus_syndactylus:6.598362):13.007584,((Gorilla_gorilla_gorilla:8.652233, Pan_troglodytes_troglodytes:8.652232):6.480222,

Pongo_pygmaeus:15.132455):4.473491):10.394055):16.811821,(Ateles_fusciceps:21.3 21300, Cebus_apella:21.321301):25.490521)

The in vivo consensus tree used for phylogenetic regressions (Chapter 3). The tree topology based on a downloaded consensus tree from the 10k Tree Project (Arnold et al. 2010). Papio cynocephalus and Ateles geoffroyi were used for Papio hamadryas-anubis-cynocephalus and Ateles fusciceps-geoffroyi-robustus hybrids. 


\section{APPENDIX B}

OSTEOLOGICAL SPECIMENS INCLUDED IN THIS STUDY FROM CAPTIVE OR UNKNOWN ORIGINS 
All individuals in this study are confirmed wild-shot specimens except those listed here.

\begin{tabular}{lllll}
\hline Species & Museum & Number & Sex & Origin \\
\hline Ateles belzebuth & AMNH & 259 & $\mathrm{~F}$ & no data \\
Ateles belzebuth & AMNH & 202194 & $\mathrm{~F}$ & zoo \\
Ateles paniscus & AMNH & 35449 & $\mathrm{M}$ & zoo \\
Erythrocebus patas & USNM & 257013 & $\mathrm{~F}$ & zoo \\
Erythrocebus patas & USNM & 238072 & $\mathrm{M}$ & zoo \\
Erythrocebus patas & USNM & 399317 & $\mathrm{M}$ & zoo \\
Gorilla gorilla & CMNH & $\mathrm{B} 1057$ & $\mathrm{M}$ & no data \\
Gorilla gorilla & CMNH & B1431 & $\mathrm{M}$ & no data \\
Nasalis larvatus & USNM & 399070 & $\mathrm{M}$ & zoo \\
Pan troglodytes & CMNH & B2746 & $\mathrm{M}$ & no data \\
Pongo abelli & USNM & 22937 & $\mathrm{~F}$ & no data \\
Pygathrix nemaeus & USNM & 536409 & $\mathrm{~F}$ & zoo \\
Symphalangus syndactylus & USNM & 519573 & $\mathrm{~F}$ & no data \\
Symphalangus syndactylus & USNM & 395514 & $\mathrm{M}$ & no data \\
Theropithecus gelada & USNM & 319992 & $\mathrm{~F}$ & zoo \\
Theropithecus gelada & USNM & 240885 & $\mathrm{M}$ & zoo \\
Theropithecus gelada & USNM & 305107 & $\mathrm{M}$ & zoo \\
Theropithecus gelada & UZIA & 8555 & $\mathrm{~F}$ & zoo \\
Theropithecus gelada & UZIA & 9300 & $\mathrm{~F}$ & zoo \\
Theropithecus gelada & UZIA & 10351 & $\mathrm{~F}$ & zoo \\
Theropithecus gelada & UZIA & 12108 & $\mathrm{~F}$ & zoo \\
Theropithecus gelada & ZMA & 11341 & $\mathrm{M}$ & died immediately after import \\
& & & & to zoo
\end{tabular}

Museums are the United States National Museum (USNM), Cleveland Museum of Natural History $(\mathrm{CMNH})$, American Museum of Natural History (AMNH), Naturalis Leiden (ZMA), and University of Zurich (UZIA). 


\section{APPENDIX C}

INSTITUTIONAL ABBREVIATIONS 
Institutional abbreviations for extant specimens:

AMNH, American Museum of Natural History, New York, NY, USA

CMNH, Cleveland Museum of Natural History, Cleveland, OH, USA

FMNH, Field Museum of Natural History, Chicago, IL, USA

KNM, National Museums of Kenya, Nairobi, Kenya

MCZ, Museum of Comparative Zoology at Harvard, Cambridge, MA, USA

MRAC, Royal Museum for Central Africa, Tervuren, Belgium

PCM, Powell Cotton Museum, Birchington, Kent, United Kingdom

USNM, United States National Museum, Washington DC, USA

UZIA, University of Zurich, Zurich, Switzerland

ZMA, Naturalis Leiden and Zoological Museum Amsterdam collections, Leiden, Netherlands

ZSM, Bavarian State Zoological Collections, Munich, Germany

Institutional abbreviations for fossil specimens:

BA, Naturhistorisches Basel, Basel, Switzerland

BMNH, British Museum of Natural History, London, United Kingdom

IGF, Museo di Storia Naturale at the Universitá degli Studi di Firenze, Florence, Italy IPS, Institut Català de Paleontologia Miguel Crusafont, Sabadell, Spain

KNM, National Museums of Kenya, Nairobi, Kenya

MUZ-M, Makerere University Zoology Museum (MUZ-M), Kampala, Uganda

RUD, the materials from the site of Rudabánya housed at the Geological Institute of Hungary, Budapest, Hungary.

YPM, Yale Peabody Museum housed at Peabody Museum at Harvard University, Cambridge, MA, USA 